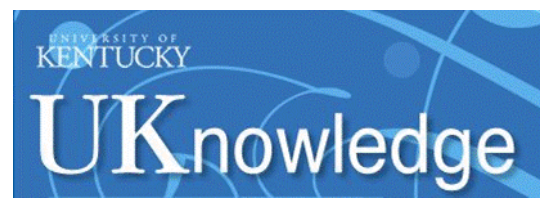

University of Kentucky

UKnowledge

\title{
LOCALIZATION OF STATIONARY SOURCE OF FLOOR VIBRATION USING THE STEERED RESPONSE POWER METHOD
}

\author{
Mohammad Royvaran \\ University of Kentucky, m.royvaran@yahoo.com \\ Author ORCID Identifier: \\ (iD) https://orcid.org/0000-0003-3018-4077 \\ Digital Object Identifier: https://doi.org/10.13023/etd.2021.368
}

Right click to open a feedback form in a new tab to let us know how this document benefits you.

\section{Recommended Citation}

Royvaran, Mohammad, "LOCALIZATION OF STATIONARY SOURCE OF FLOOR VIBRATION USING THE STEERED RESPONSE POWER METHOD" (2021). Theses and Dissertations--Civil Engineering. 112. https://uknowledge.uky.edu/ce_etds/112

This Doctoral Dissertation is brought to you for free and open access by the Civil Engineering at UKnowledge. It has been accepted for inclusion in Theses and Dissertations--Civil Engineering by an authorized administrator of UKnowledge. For more information, please contact UKnowledge@lsv.uky.edu. 


\section{STUDENT AGREEMENT:}

I represent that my thesis or dissertation and abstract are my original work. Proper attribution has been given to all outside sources. I understand that I am solely responsible for obtaining any needed copyright permissions. I have obtained needed written permission statement(s) from the owner(s) of each third-party copyrighted matter to be included in my work, allowing electronic distribution (if such use is not permitted by the fair use doctrine) which will be submitted to UKnowledge as Additional File.

I hereby grant to The University of Kentucky and its agents the irrevocable, non-exclusive, and royalty-free license to archive and make accessible my work in whole or in part in all forms of media, now or hereafter known. I agree that the document mentioned above may be made available immediately for worldwide access unless an embargo applies.

I retain all other ownership rights to the copyright of my work. I also retain the right to use in future works (such as articles or books) all or part of my work. I understand that I am free to register the copyright to my work.

\section{REVIEW, APPROVAL AND ACCEPTANCE}

The document mentioned above has been reviewed and accepted by the student's advisor, on behalf of the advisory committee, and by the Director of Graduate Studies (DGS), on behalf of the program; we verify that this is the final, approved version of the student's thesis including all changes required by the advisory committee. The undersigned agree to abide by the statements above.

Mohammad Royvaran, Student

Dr. Douglas Bradley Davis, Major Professor

Dr. Lindsey Sebastian Bryson, Director of Graduate Studies 


\title{
LOCALIZATION OF STATIONARY SOURCE OF FLOOR VIBRATION USING THE STEERED RESPONSE POWER METHOD
}

\author{
DISSERTATION \\ A dissertation submitted in partial fulfillment of the \\ requirements for the degree of Doctor of Philosophy in the \\ College of Engineering \\ at the University of Kentucky \\ By \\ Mohammad Royvaran \\ Lexington, Kentucky \\ Director: Dr. Douglas Bradley Davis, Associate Professor of Civil Engineering \\ Lexington, Kentucky \\ 2021
}

Copyright (C) Mohammad Royvaran 2021

https://orcid.org/0000-0003-3018-4077 


\section{ABSTRACT OF DISSERTATION}

\section{LOCALIZATION OF STATIONARY SOURCE OF FLOOR VIBRATION USING THE STEERED RESPONSE POWER METHOD}

If the generated vibration in a building exceeds the acceptable limit design for a floor system, it is necessary to identify the source of vibration, a process known as localization. The objective of this study is the localization of stationary vibration sources, and the approach used is the steered response power (SRP) method. This method has already been shown to work well for wireless and acoustical applications to locate transmitter and sound sources, respectively. To the writer's knowledge, this study is the first application of the SRP method to locate vibration sources using floor vibration measurements. However, because waves behave differently when propagated through a concrete floor as opposed to the air, this method has been significantly modified for the application presented herein.

The key and prerequisite parameter for most vibration-sensing-localization approaches is wave propagation speed (WPS). The accuracy of these approaches therefore depends on the accuracy of the WPS estimate. The WPS of a concrete floor system is a function of parameters with high variability due to the mechanical and dynamic properties of the floor. This makes the task of vibration-sensing-localization challenging for the aforementioned approaches. The SRP method has been employed because it is based on an algorithm to post-process all received signals together and such structural variability is less likely to affect the accuracy; therefore, the SRP method is more robust.

Most localization approaches are based on ideal wave propagation, e.g., constant propagation speed in all directions and vibration energy decreasing predictably as the source-sensor distance increases. However, such ideal propagation does not occur in many real-world structural systems such as a concrete floor. In this study, the WPS was estimated empirically in orthogonal directions using the cross-correlation function. The SRP method used herein was adopted to use the estimated WPS in orthogonal directions as an input parameter and then automatically interpolating the corresponding propagation speed for all other directions. This is another advantage of this method over existing methods.

The experiment was conducted on the second floor of a full-scale, concrete-framed building at the University of Kentucky. The WPS was estimated in orthogonal directions using an electrodynamic shaker and seven accelerometers. The shaker applied an excitation force and acted as the source of vibration, and the accelerometers were put in various locations on the floor and measured the response. Using the estimated WPS and corresponding measurement data, the SRP method was able to locate the vibration source within $2.0 \mathrm{~m}$ in a floor approximately $13.4 \mathrm{~m}$ by $8.4 \mathrm{~m}$ in size. 
KEYWORDS: Localization, Floor Vibration, Steered Response Power, Time Difference of Arrival, Wave Propagation Speed, Time Delay 


\title{
LOCALIZATION OF STATIONARY SOURCE OF FLOOR VIBRATION USING STEERED RESPONSE POWER METHOD
}

\author{
By \\ Mohammad Royvaran
}

Dr. Douglas Bradley Davis

Director of Dissertation

Dr. Lindsey Sebastian Bryson

Director of Graduate Studies

$07 / 27 / 2021$

Date 


\section{ACKNOWLEDGMENTS}

There are many people who have helped me during this doctoral program. First and foremost, I would like to express my deep gratitude to my advisor and committee chair, Dr. Brad Davis. He has been an amazing mentor and good friend to me over the past five years and has always encouraged me to do my best and feel confident in my abilities. His knowledge, experience, and guidance have always given me a positive energy in my research and have helped me develop new skills and learn valuable lessons, both personally and academically. Without his assistance, I would not have been able to complete this dissertation. I also want to thank him for his support in financing my studies.

Furthermore, I would like to give a special thanks to Dr. Kevin Donohue for his insight beyond the field of Civil Engineering. In the past three years, I have had many meetings with him, and he has always been very encouraging and guided me in the right direction. I got the idea of localization from his class, and his excellent feedback has improved my research.

I am grateful to Dr. Issam Harik and Dr. John Baker whose instruction during my doctoral program was essential to this dissertation. I would also like to thank both Dr. Samantha Wright for giving me permission to use her computer lab for all my vibration testing and Dr. Andrew Beutel for his diligent proofreading of this dissertation.

Finally, and most importantly, I would like to thank my wife, Mondana, my mother and father, and all my brothers and sisters for their unconditional love during every step of my education. I cannot put into words how grateful I am to all of you. I would also like to thank my uncle, Dr. Manouchehr Katanbaf, and all the wonderful friends I have made during the past five years who truly made my time in Lexington enjoyable. 
ACKNOWLEDGMENTS .................................................................................. ii

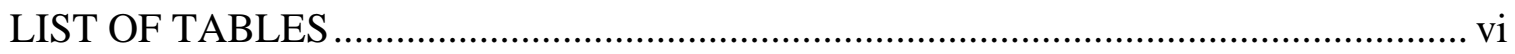

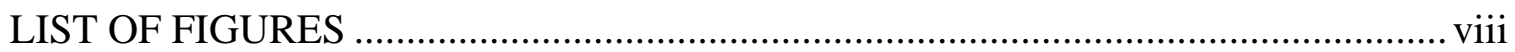

CHAPTER 1. INTRODUCTION ............................................................................... 1

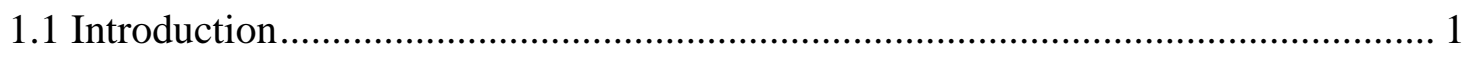

1.2 Literature Review................................................................................................... 3

1.2.1 Floor Vibration Overview - Source, Path, and Receiver...................................... 3

1.2.2 Vibration Path and Wave Propagation Basics ................................................... 4

1.2.3 Near Field vs. Far Field Region ................................................................... 6

1.2.4 Noise and Distortion Effects........................................................................ 8

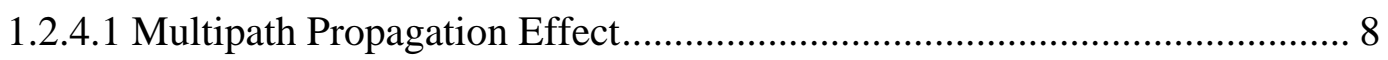

1.2.4.2 Attenuation................................................................................. 9

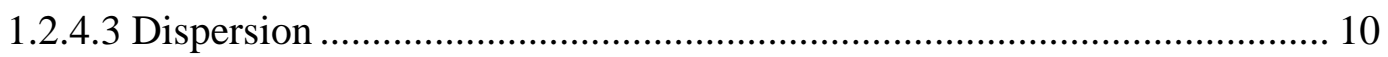

1.2.5 Localization Methods................................................................................ 11

1.3 Research Objectives....................................................................................... 19

CHAPTER 2: STEERED RESPONSE POWER METHOD........................................... 22

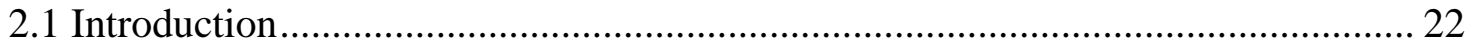

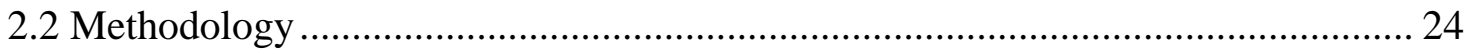

2.3 Estimation of Major Parameter, Wave Propagation Speed ...................................... 43

2.3.1 Previous Studies and Challenges in Time Delay Estimation............................ 45

2.3.2 Time Delay Estimation Using Time-Domain Approach ................................... 46

2.3.3 Time Delay Estimation Using the Frequency-Domain Approach..................... 47

CHAPTER 3: EXPERIMENTAL PROGRAM............................................................... 49

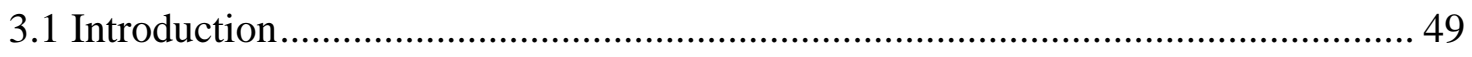

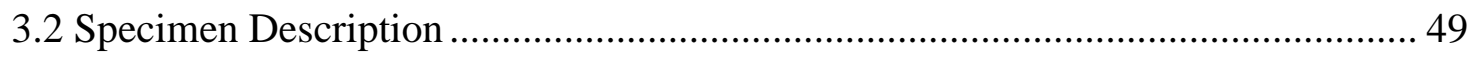

3.3 Dynamic Testing Equipment ................................................................................ 51

3.3.1 Electrodynamic Shaker .................................................................................. 51

3.3.2 Multi-Channel Spectrum Analyzer ................................................................. 53

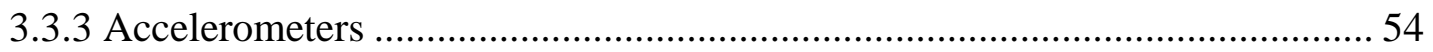

3.4 Determination of Frequency Response Function..................................................... 55

3.4.1 Theory of Frequency Response Function ........................................................ 56 
3.4.2 Estimation of Frequency Response Function (FRF).................................. 57

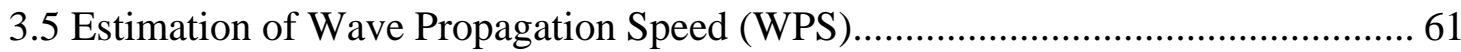

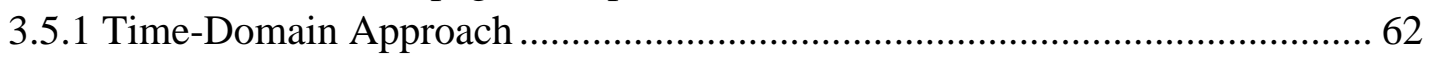

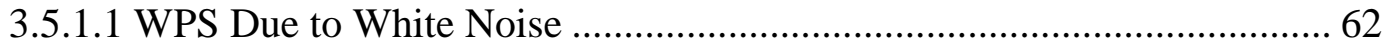

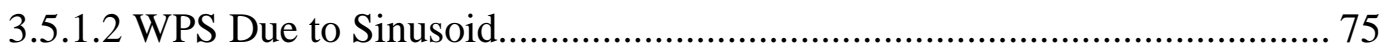

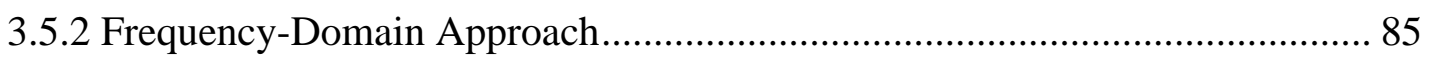

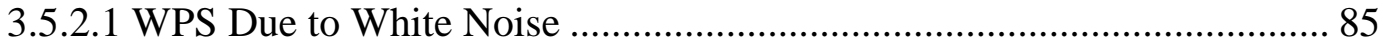

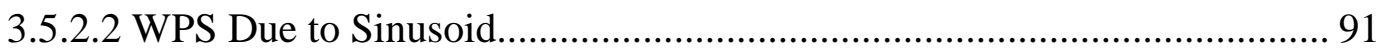

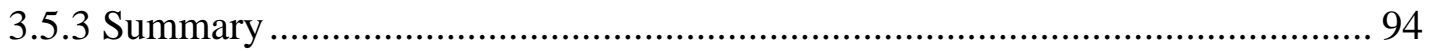

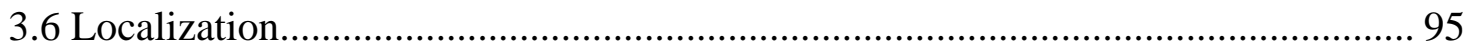

3.6.1 Localization of White Noise Excitation....................................................... 97

3.6.2 Localization of Sinusoid Excitation............................................................... 110

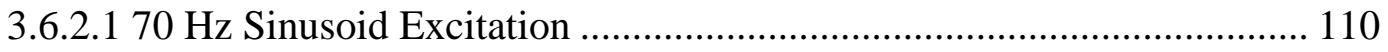

3.6.2.2 $90 \mathrm{~Hz}$ Sinusoid Excitation ................................................................. 117

3.6.2.3 110 Hz Sinusoid Excitation ............................................................... 122

CHAPTER 4: SUMMARY, CONCLUSION, AND RECOMMENDATION FOR

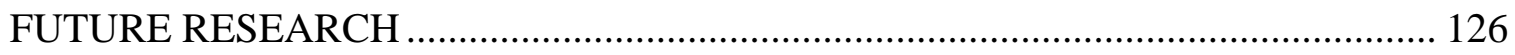

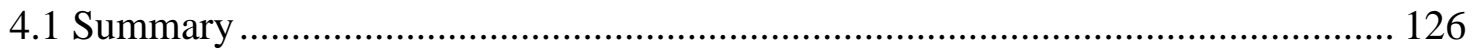

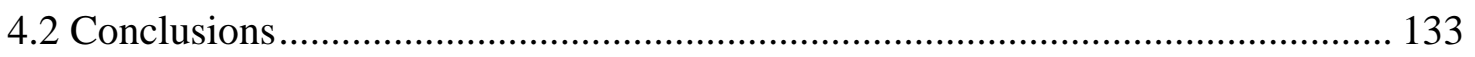

4.3 Recommendations for Future Research ....................................................... 135

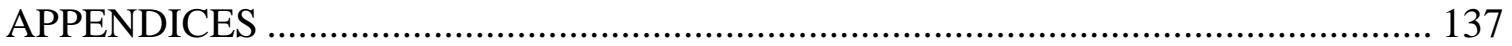

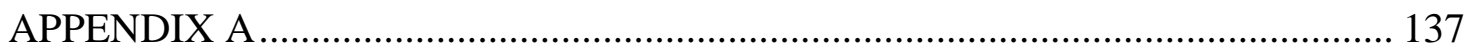

A.1 Localization of White Noise Excitation (Regarding Section 3.6.1) ............... 137

A.2 Localization of $70 \mathrm{~Hz}$ Sinusoid Excitation (Regarding Section 3.6.2.1) ......... 144

A.3 Localization of $90 \mathrm{~Hz}$ Sinusoid Excitation (Regarding Section 3.6.2.2) ......... 147

A.4 Localization of $110 \mathrm{~Hz}$ Sinusoid Excitation (Regarding Section 3.6.2.3) ....... 150

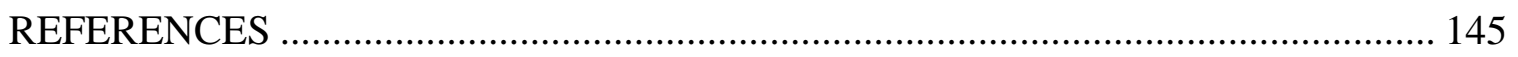

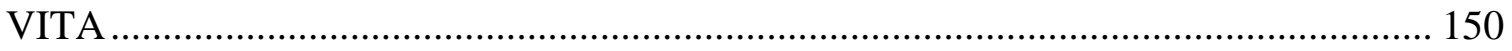




\section{LIST OF TABLES}

TABLE 2.1: List Of SiMUlationS AND THEIR DETAILS....................................................... 27

TABLE 2.2: EFFECT OF B ON INVERSE DiSTANCE WEIGHTING ........................................... 42

TABLE 2.3: WPS ESTIMATION IS SOME STUDIES …………............................................ 43

TABle 3.1: Some CAPABILITIES Of APS ELECTRO-SEIS Model 400 …........................ 51

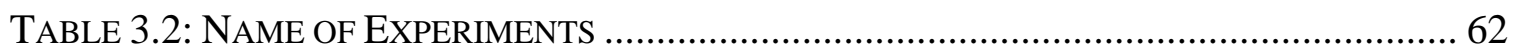

Table 3.3: Estimated Propagation Speed (CASE 1: Entire Signal With No

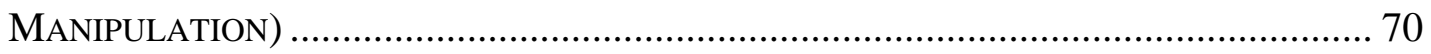

Table 3.4: Estimated Propagation Speed (CASE 2: Entire Signal + Filtered in FREQUENCY RANGE OF INTEREST)................................................................... 71

Table 3.5: Estimated Propagation Speed (CASE 3: Signal Gated in Time of INTEREST + FILTERED IN FREQUENCY RANGE OF INTEREST) .................................... 72

Table 3.6: Estimated Propagation Speed (Case 4: Signal Gated in Time of INTEREST + FILTERED IN FREQUENCY RANGE OF INTEREST + PARTIAL WHITENING) 74

TABLE 3.7: PROPAGATION SPEEd RESUltS FOR DifFERENT SHAKER POWER..................... 75

TABLE 3.8: LIST OF TESTS WITH SINUSOID EXCITATION SIGNALS ....................................... 76

Table 3.9: Estimated Propagation Speed (CASE 1: Entire Signal With No

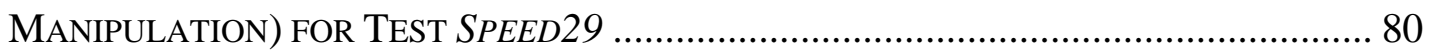

Table 3.10: Estimated Propagation Speed (CASe 2: Entire Signal + Filtered in the FREQUENCY RANGE OF INTEREST) FOR TEST SPEED29 ........................................... 81

Table 3.11: Estimated Propagation SpeEd (CASE 3: Signal Gated in Time of INTEREST + FILTERED IN THE FREQUENCY RANGE OF INTEREST) FOR TEST SPEED29 82

Table 3.12: Estimated Propagation Speed (CASE 4: Signal Gated in Time of INTEREST + FILTERED IN THE FREQUENCY RANGE OF INTEREST + PARTIAL WHITENING) FOR TEST SPEED29.

TABLE 3.13: SpeEd Propagation Estimation FOR SPEED29 AND SPEED30 DuE TO $70 \mathrm{~Hz}$ SINUSOID EXCITATION. 84

Table 3.14: SpeEd Propagation Estimation for SPEED31 and SPEED32 Due to $90 \mathrm{~Hz}$ SINUSOID EXCITATION. 84

TABLE 3.15: SpeEd Propagation Estimation For SPEED33 AND SPEED34 Due to 110 HZ SINUSOID EXCITATION

TABle 3.16: EfFEct of Defined Phase Tolerance Threshold on Estimated SPEed FOR A2 ACCELEROMETER FOR TEST SPEED25

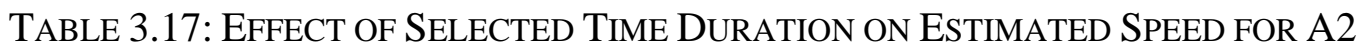
ACCELEROMETER FOR TEST SPEED25. 89

TABLE 3.18: Estimated SPEED FOR FREQUENCy APPROACH Due to White NoISE EXCITATION. 91

TABLE 3.19: EstimATED SPEED FOR THE ENTIRE SigNAL IN DifFERENT FREQUENCY RANGES DUE TO $70 \mathrm{~Hz}$ SINUSOID EXCITATION 
TABle 3.20: Estimated SPEed For the EnTiRe Signal in DifFERENT Frequency RANGES DUE TO 90 Hz SINUSOID EXCITATION .......................................................... 93

TABLE 3.21: Estimated SPEED For the ENTIRE Signal In DifFERENT FREQUENCY RANGES DUE TO 110 Hz SINUSOID EXCITATION …………….................................. 93

TABle 3.22: EFFECT OF SELECTEd Time DURATION ON Estimated SPEEd FOR A2

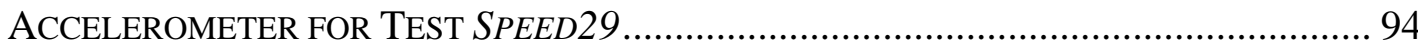

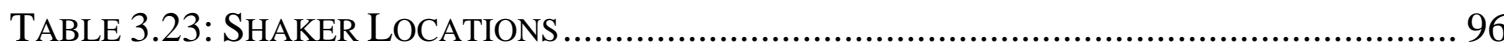

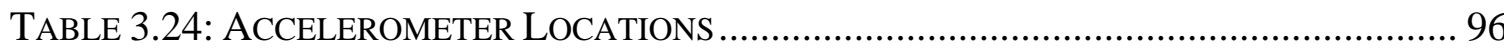

TABLE 3.25: LIST OF WhITE NoISE EXCITATION TESTS ……………………………….... 97

TABLE 3.26: ACCELEROMETER CONTRIBUTIONS WITH RESPECT TO DIFFERENT DECAYING POWERS IN EqUATION 2.12 AT COORDINATES $X=3.7$ M AND $Y=3.7$ M FOR THE TEST OHR2ND-L1-1 102

TABle 3.27: Average WPS IN X- AND Y-Directions For the White Noise EXCitATION BASED ON TABLE 3.7 103

TABle 3.28: LOCALIZATION ERRORS FOR THE FIRST, SECOND, AND THIRD HigheSt PEAKS

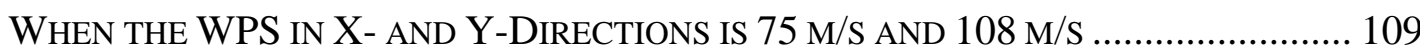

TABLE 3.29: LIST OF TESTS WITH 70 Hz SINUSOID EXCITATION ........................................ 110

TABLE 3.30: AVERAGE WPS IN X- AND Y-DiRECTIONS FOR THE 70 Hz SinUSOID EXCITATION 111

TABLE 3.31: LOCALIZATION ERRORS FOR THE FIRST, SECOND, AND THIRD HigheST PEAKS WHEN THE WPS IN X- AND Y-DIRECTIONS IS $37 \mathrm{M} / \mathrm{s}$ AND $61 \mathrm{M} / \mathrm{s}$............................. 117

TABLE 3.32: LIST OF TESTS WITH 90 Hz SINUSOID EXCITATION ………………………..... 117

TABLE 3.33: AVERAge WPS IN X- AND Y-DireCtions FOR THE 90 Hz SinUSOID

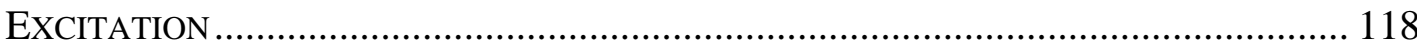

TABLE 3.34: LOCALIZATION ERRORS FOR THE FIRST, SECOND, AND THIRD HigHEST PEAKS WHEN THE WPS IN X- AND Y-DiRECTIONS IS $47 \mathrm{M} / \mathrm{s}$ AND $74 \mathrm{M} / \mathrm{s}$.................................. 121

TABLE 3.35: LiST OF TESTS WITH 110 Hz SINUSOID EXCITATION ...................................... 122 TABLE 3.36: AvERAGE WPS IN X- AND Y-DiRECTIONS FOR 110 Hz SinUSOID EXCITATION

TABLE 3.37: LOCALIZATION ERRORS FOR THE FIRST, SECOND, AND THIRD HighEST PEAKS WHEN THE WPS IN X- AND Y-DIRECTIONS IS $48 \mathrm{M} / \mathrm{S}$ AND $105 \mathrm{M} / \mathrm{s}$.......................... 124

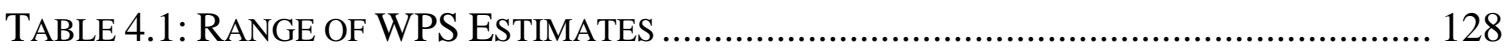

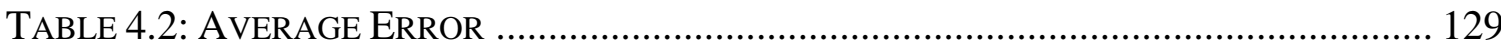




\section{LIST OF FIGURES}

Figure 1.1: P-WaVe, S-WAVE, And LAmb WaVe in Vertical and Horizontal Plane

(РHOTO BY DONDURUR 2018) ................................................................................ 5

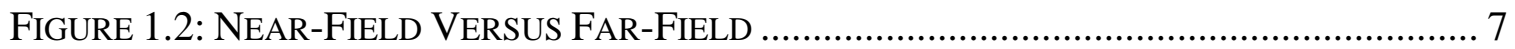

FigURE 1.3: EFFECT OF DisPERSION ON SiMULATED RECEIVED SignALS AT DisTANCES 5, 10, 15, AND 20 M (РHOTO BY BAHROUN ET AL. 2014).............................................. 11

Figure 1.4: LoCALization of Epicenter (Credit: NATional Atlas, U.S. GeOlogical

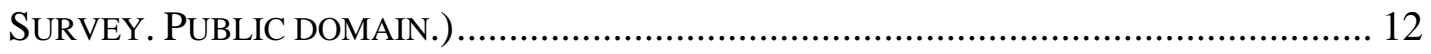

FIgURE 1.5: VibRATION SOURCE LOCALIZATION USING RSS METHOD ........................... 14 Figure 1.6: Source Excites the First Mode, SENSOR 2 REPORTS A Higher Response

FigURE 1.7: VibRATION SOURCE LOCALIZATION USING TDOA ALGORITHM ................... 16

FigURE 2.1: SCHEMATIC VIEW OF EQUATION 2.7 ........................................................ 26

Figure 2.2: SimUlation 1 CONFIGURATION AND EXCITATION AND RECEIVED SigNALS . 28

FiguRE 2.3: CANDIDATE LOCATIONS AS THE SOURCE OF VibRATION ............................... 28

Figure 2.4: VisUal Simulation Of THE SRP METHOD (Simulation 1)......................... 30

Figure 2.5: RESUltS OF Visual Simulation of THE SRP METHOD (Simulation 1)..... 31

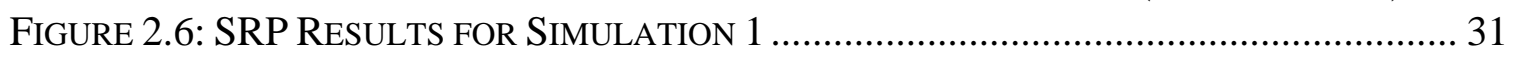

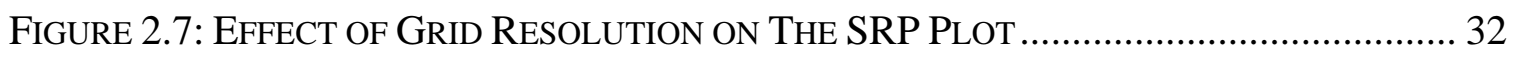

Figure 2.8: EFFECT OF SAMPLING FREQUENCY RATE ON TIME SHIFT .............................. 33

FiguRE 2.9: EFFECT OF SAMPLING FREQUENCY ON SRP RESULTS ................................. 33

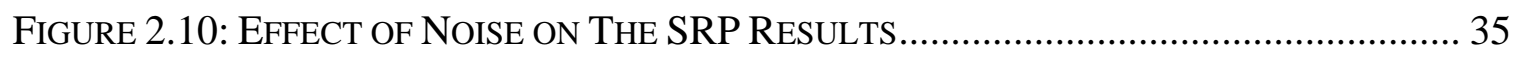

Figure 2.11: SOURCE LOCATED AT BISECTOR OF TWO ACCELEROMETERS AND

CORRESPONDING SR PLOT (SIMULATION 9) ......................................................... 36

Figure 2.12: SOURCE LOCATED AT BisECTOR OF TWO ACCELEROMETERS AND

CORRESPONDING SRP PLOT (SIMULATION 10) ..................................................... 37

Figure 2.13: SOURCE LOCATED NOT Placed AT BiseCtor of Two ACCELEROMETERS

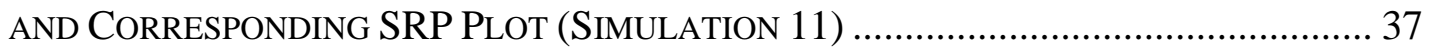

Figure 2.14: EXCITATION FORCE FOR SIMULATIONS 12, 13, 14, AND 15 ........................ 38

FIGURE 2.15: USE OF TWO ACCELEROMETERS AND SRP PLOT FOR SINUSOID EXCITATION

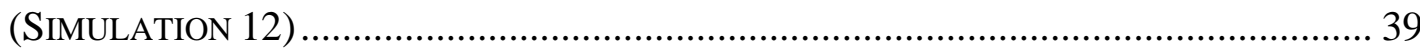

Figure 2.16: UsE OF THREE ACCELEROMETERS AND SRP PLOT FOR SiNUSOID EXCITATION

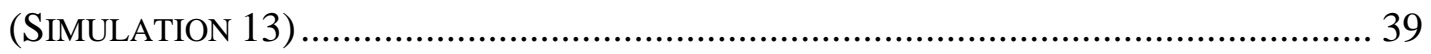

FIgURE 2.17: USE OF FOUR ACCELEROMETERS AND SRP PLOT FOR SINUSOID EXCITATION

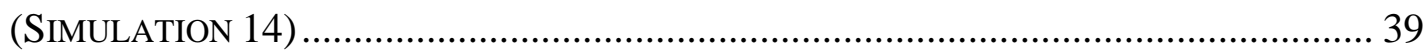

Figure 2.18: EFFECT OF IRREGULAR DiSTRIBUTION OF ACCELEROMETERS IN SRP PLOTS FOR SINUSOID EXCITATION (SIMULATION 15) .................................................... 40

Figure 2.19: PERFormance Of SRP AlgORIthM With RANDOM Noise (Simulation 16)

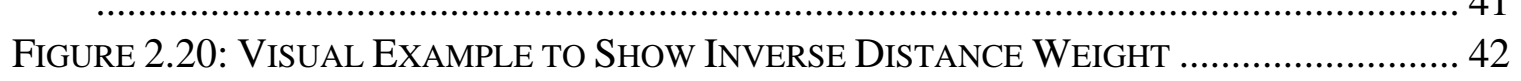


FigURE 2.21: EFFECT OF INACCURATE WPS ESTIMATION ON THE ACCURACY OF SRP Algorithm (SimUlation 17)

Figure 3.1: Structural DraWing of the SECOND FloOR, OHR BUILDING, UNiVERSiTy OF KENTUCKY. 50

Figure 3.2: Section View in the North-South Direction Along With COLUMn-Line E AND F 50

FIGURE 3.3: APS ELECTRO-SEIS MODEL 400 SHAKER ................................................. 52

Figure 3.4: Multi-ChanNel SPECTRUM ANALYZER, NV-Gate SoftWARE PROGRAM,

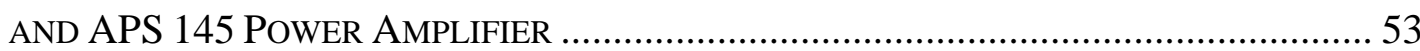

FIGURE 3.5: 393C PCB PIEZOTRONICS ........................................................................ 54

FIGURE 3.6: EQUIPMENT SETUP .............................................................................. 55

FigURE 3.7: DRAWING FOR THE TESTED FLOOR AND LOCATION OF ACCELEROMETERS AND

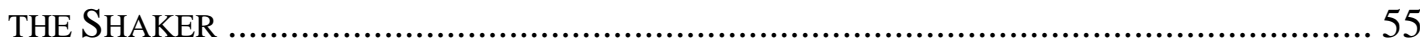

Figure 3.8: PoSITION OF ACCELEROMETERS AND THE SHAKER ......................................... 56

Figure 3.9: TIME-DOMAIN AND CORRESPONDING FREQUENCY-Domain OF White NoISE

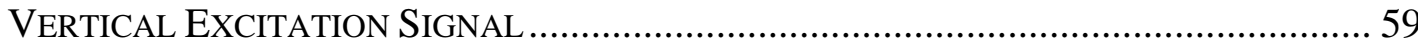

Figure 3.10: TIME-DOMAIN AND CORRESPONDING FREQUENCY-DOMAIN OF A1 RESPONSE DUE TO THE White NoISE VERTICAL EXCITATION SignaL........................................ 59

FIGURE 3.11: FRF AND COHERENCE FOR SET OF ACCELEROMETERS IN X- AND Y-

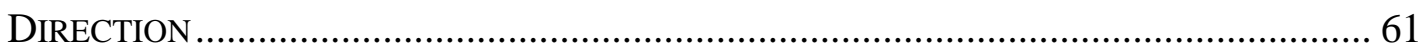

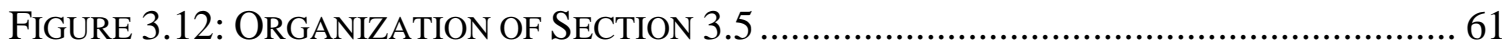

FIGURE 3.13: CONFIGURATION OF THE SHAKER AND ACCELEROMETERS FOR WPS

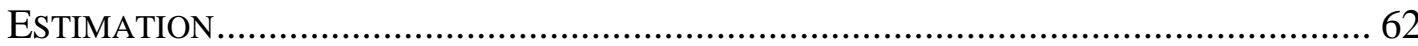

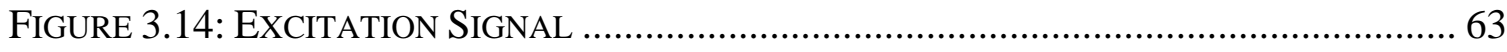

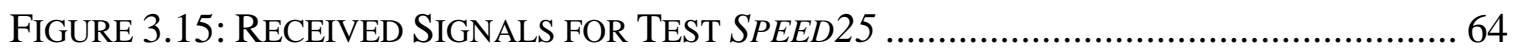

FigURE 3.16: PSD OF EXCITATION AND A2 ACCELEROMETER FOR THE TEST SPEED25 .... 65

Figure 3.17: ORder of Processing TeChNiques AVAilable IN EACH CASE .................. 66

Figure 3.18: CROSS-CORRELATION BETWEEN EXCITATION AND A2 ACCELEROMETER.... 67

Figure 3.19: Close VIEW OF CROSS-CORRELATION BETWEEN EXCITATION AND A2

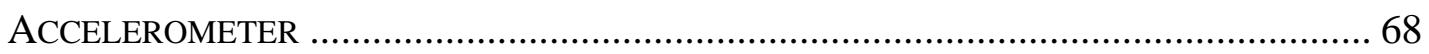

Figure 3.20: Close VIEW OF CROSS-CORRELATION BETWEEN EXCITATION AND A3

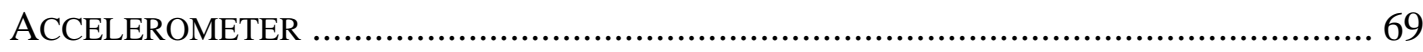

Figure 3.21: ApPLIEd FILTER IN FREQUENCY-DOMAIN AND A2 ACCELEROMETER BEFORE

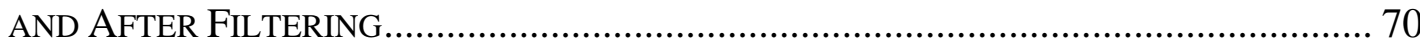

FIGURE 3.22: TIME OF INTEREST IN CASE 3 ................................................................ 72

FiguRE 3.23: EFFECT OF PARTIAL WHITENING ON TIME- AND FREQUENCY-DOMAIN FOR A2 ACCELEROMETER …………..................................................................... 74

Figure 3.24: (A) EXCitATION Signal FOR SinUSOId of 70 Hz AND (B) ITS ClOSEd VieW FOR TEST SPEED29.......................................................................................... 76

FiguRE 3.25: RECEIVED SigNALS DUE TO SINUSOID EXCITATION WITH FREQUENCY OF 70

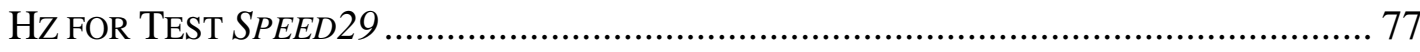


FiguRE 3.26: PSD FOR (A) SinUSOID EXCITATION WITH FREQUENCY OF 70 Hz AND (B) A2

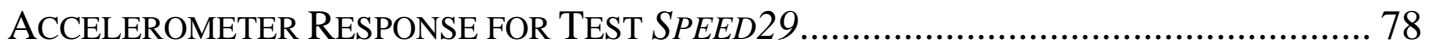

FIGURE 3.27: CROSS-CORRELATION FUNCTION BETWEEN EXCITATION AND A2

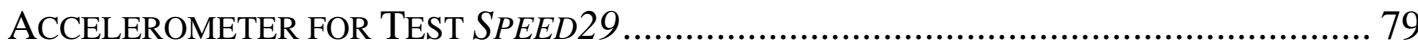

Figure 3.28: ClOSE VIEW OF CROSS-CORRELATION FUNCTION BETWEEN EXCITATION AND A2 ACCELEROMETER FOR TEST SPEED29. 80

Figure 3.29: (A) The WAy Signal FiLtered in Frequency-Domain AND (B) The EFFECT OF FILTERING IN TIME-DOMAIN SignAl of A2 ACCELEROMETER FOR TEST SPEED29 81

Figure 3.30: SignAl GATED IN TIME OF INTEREST FOR A2 ACCELEROMETER FOR TEST SPEED29 82

Figure 3.31: EFFECT OF PARTIAL WHitening on (A) TIME-DOMAIN AND (B) FREQUENCYDOMAIN FOR A2 ACCELEROMETER FOR TEST SPEED29. 83

Figure 3.32: CROSS-SPECTRUM BETWEEN EXCITATION AND A2 ACCELEROMETER FOR CASE 1 86

Figure 3.33: TiME OF INTEREST FOR EXCITATION AND A2 ACCELEROMETER FOR CASE 288

Figure 3.34: CROSS-SPECTRUM BETWEEN EXCITATION AND A2 ACCELEROMETER FOR CASE 2 88

Figure 3.35: Negative Estimated SPEEd FOR Test SPEED26 AND FOR A5 ACCELEROMETER FOR TIME OF INTEREST FROM 11-13 SEC 89

Figure 3.36: CROSS-SPECTRUM BETWEEN EXCITATION AND A2 ACCELEROMETER FOR THE FREQUENCY RANGE FROM 80-180 Hz FOR CASE 3, FOR TEST SPEED25 AND TIME OF INTEREST FROM 10.3-12.3 SEC .... 90

Figure 3.37: Tested AREa Between Column Lines 3-4 AND F-E .............................. 95

Figure 3.38: Photos of The Origin AND The SHAKer IN The TESTEd FloOR .............. 96

Figure 3.39: Test Setup for Tests When the Shaker WAS Placed AT L1................ 98

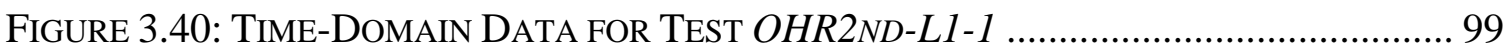

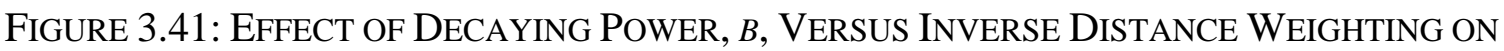

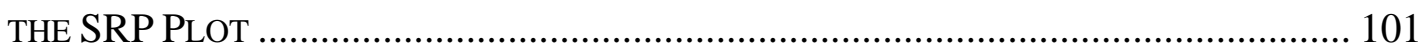

FigURE 3.42: LOCALIZATION ERROR PLOT WITH RESPECT TO WPS IN X- AND Y-

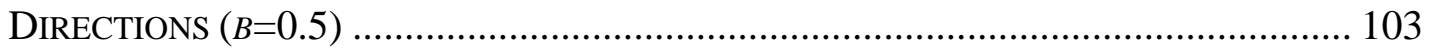

FIGURE 3.43: CONSIDERED TWO-SECOND WAVEFORM FROM 10.3-12.3 SEC FOR ALL ACCELEROMETERS FOR THE TEST OHR2ND-L1-1 ............................................. 104

Figure 3.44: LOCALIZATION ERROR PLOT WITH RESPECT TO WPS IN X- AND YDiRECTIONS CONSIDERING TWO-SECOND WAVEFORM FROM 10.3-12.3 SEC $(B=0.5)$

Figure 3.45: LOCALIZATION ERROR PLOT WITH RESPECT TO WPS IN X- AND YDiRECTIONS CONSIDERING FIVE-SECOND WAVEFORM FROM 15-20 SEC $(B=0.5) \ldots 105$

FiguRE 3.46: EFFECT OF LENGTH OF TIME DURATION ON THE SRP PlOTS $(B=0.5) \ldots \ldots . . . .106$ Figure 3.47: EFFECT OF FILTERING ON LOCALIZATION ERROR PLOTS WITH RESPECT TO WPS IN X- AND Y-DIRECTIONS CONSIDERING TWO-SECOND WAVEFORM FROM 15-17 $\operatorname{SEC}(B=0.5)$ 107 
Figure 3.48: LOCALIZATION ERROR PLOT WITH RESPECT TO WPS IN X- AND YDIRECTIONS WHEN CONSIDERING A TWO-SECOND WAVEFORM FROM 15-17 SEC AND FILTERING IN A FREQUENCY RANGE OF 5-200 Hz ( $B=0.5)$. 108

FIGURE 3.49: SRP PLOTS WHEN CONSIDERING A TWO-SECOND WAVEFORM FROM 15-17 SEC AND FILTERING IN A FREQUENCY RANGE OF 5-200 Hz $(B=0.5)$........................ 109

Figure 3.50: WAVEFORMS FOR ALL ACCELEROMETERS FOR TEST OHR2ND-L1-7........ 111

Figure 3.51: THE EFFECT OF INVERSE DisTANCE WEIGHTING ON LOCALIZATION ERROR WHEN CONSIDERING A TWO-SECOND WAVEFORM FROM 15-17 SEC $(B=0.5) \ldots \ldots . . . .112$

Figure 3.52: EFFECT OF INVERSE DistANCE WEIGHTING IN THE SRP PLOT WHEN CONSIDERING A TWO-SECOND WAVEFORM FROM 15-17 SEC .................................. 114

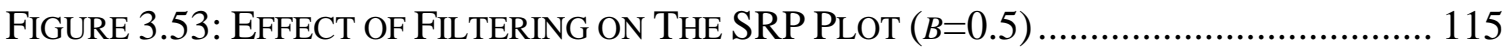

FIGURE 3.54: SRP PLOTS WHEN CONSIDERING A TWO-SECOND WAVEFORM FROM 15-17 SEC AND FILTERING IN A FREQUENCY RANGE OF 60-80 Hz $(B=0.5)$...................... 116

Figure 3.55: LOCALIZATION ERROR WHEN CONSIDERING A TWO-SECOND WAVEFORM FROM 15-17 SEC AND FILTERING IN A FREQUENCY RANGE OF 60-80 Hz ( $B=0.5) \ldots . .116$

Figure 3.56: EFFECT OF INVERSE DistanCE WEIGHTING ON THE SRP PlOTS WHEN CONSIDERING A TWO-SECOND WAVEFORM FROM 15-17 SEC 119

FIGURE 3.57: SRP PLOTS WHEN CONSIDERING A TWO-SECOND WAVEFORM FROM 15-17

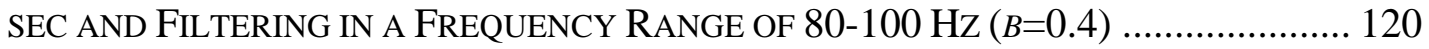

Figure 3.58: LOCALIZATION ERROR PlOTS WHEN CONSIDERING A TWO-SECOND WAVEFORM FROM 15-17 SEC AND FILTERING IN A FREQUENCY RANGE OF 80-100 Hz

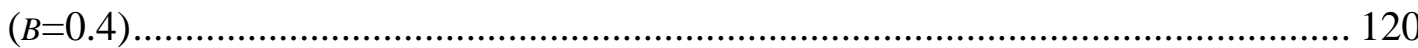

FIGURE 3.59: RESPONSE TO THE 90 Hz SiNUSOID EXCITATION................................... 121

FIGURE 3.60: SRP PLOTS WHEN CONSIDERING A TWO-SECOND WAVEFORM FROM 15-17 SEC AND FILTERING IN A FREQUENCY RANGE OF 100-120 Hz ( $B=0.5)$.................... 123

Figure 3.61: LOCALIZATION ERROR PLOTS WHEN CONSIDERING A TWO-SECOND WAVEFORM FROM 15-17 SEC AND FILTERING IN A FREQUENCY RANGE OF 100-120 Hz

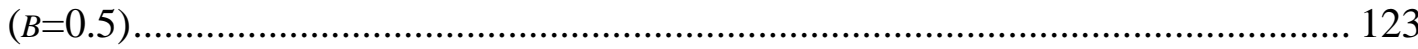

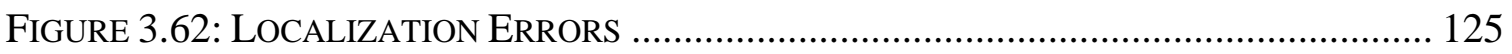

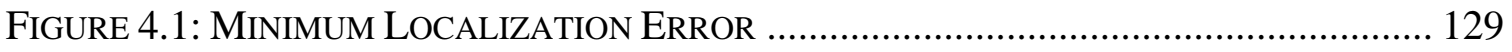

FiguRE 4.2: EXCITATION AND RECEIVED ACCELERATIONS FOR TEST OHR2ND-L4-2 (SHAKER AT L4) ..................................................................................... 130

FIGURE 4.3: EXCITATION AND RECEIVED ACCELERATIONS FOR TEST OHR2ND-L1-2

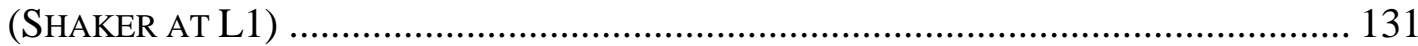

Figure 4.4: L2 LOCATION Versus BiseCtORs BETWEEN THE ACCELEROMETERS ......... 132 Figure 4.5: Applied ForCE VERSUS RECEIVED RESPONSES FOR TEST OHR2ND-L2-7 .. 133 


\section{CHAPTER 1. INTRODUCTION}

\subsection{Introduction}

In a building, when the floor vibration level exceeds the tolerance or acceptance limit, identification of the source of vibration and mitigation of generated vibration need to be performed. The objective of this study is to develop a practical method for identifying and locating the source of vibration, a process known as vibration localization, for elevated concrete floor systems. The method will be based on the steered response power (SRP) method that has been successfully used as a signal-processing technique for electromagnetic and acoustical applications to locate the transmitter or sound source, respectively. To the writer's knowledge, this study is the first application of the SRP method to surface-wave vibrations in structures to locate the source of vibration. In the SRP approach, a set of potential source locations are defined. The received acceleration signals are shifted and summed, and the steered power associated with each candidate source location is computed. The location with the maximum steered power corresponds to the most likely source location.

All floor systems can vibrate when subjected to an applied dynamic load due to machinery, e.g., chillers, fans, HVAC units, and water pumps. The method described in this study is applicable and beneficial when the generated vibration exceeds the limit. Vibration limits can be associated with either human comfort (e.g., a patient in a hospital), animals for research (for example, vivarium room), or a sensitive instrument item such as a magnetic resonance imager (MRI) or powerful microscope. The excessive vibration level can cause complaints and inconvenience for occupants or improper use of equipment sensitive to vibration. This study focuses on the localization of a stationary vibration source using floor vibration measurements. In a design application, the next step after localization is often the mitigation of excessive vibration, which is beyond the scope of this study.

A practical example is when an occupant runs on a treadmill in an unknown unit in an apartment building and causes vibration that is uncomfortable to adjacent neighbors on 
the same floor. As another example, Tigli (2014) presented his study of vibration on the fourth floor of a ten-story hospital building where excessive vibration due to an unknown source disrupted the use of a surgical microscope in an operating room for neurosurgery. In this example, the water pomp was identified as the stationary vibration source. Correct identification of the vibration source using vertical floor vibration measurements would be helpful in such situations.

The underlying motivation behind this study is that vibration serviceability is becoming more critical because there is more potential for modern floors to vibrate with higher amplitudes. Shifting away from more traditional building construction - with heavy cast-in-place concrete — to high-strength and lighter construction materials, along with modern design methods, opens the way for lighter and longer spans. Therefore, floors often have less mass and lower natural frequencies, increasing the possibility of floor vibration problems. Moreover, using an open floor layout with fewer full-height nonstructural partitions and lighter furniture reduces damping, worsening the problem. Serviceability problems can cause costly remodeling or revision of the structural design of a floor (Ebrahimpour et al. 2005).

In addition, due to advancing technology, smart buildings equipped with various sensing devices, such as vibration sensors, are becoming more common. Compared to traditional buildings, smart buildings provide improved health, human comfort, energy consumption, structural health monitoring, safety, and security in residential, commercial, and health facility buildings (Lee et al. 2009; Schloemann et al 2015; Mirshekari et al. 2016; Drira et al. 2019; Hormozabad et a. 2021). It is expected that vibration source localization will be essential in smart buildings.

This dissertation is organized as follows. Chapter 1 discusses previous research related to this study and describes some terminology and definitions employed. Chapter 2 provides the methodology and equations needed for the SRP method and the prerequisite key parameter: time delay. Other parameters that affect the accuracy and quality of the SRP method are discussed. In Chapter 3, the performance of the SRP method in a fullscale building and the use of equations mentioned in Chapter 2 are described. Then, the overall conclusions and also future research to expand this study are explained in Chapter 4. 


\subsection{Literature Review}

This section describes an overview of floor vibration and also covers some terminology not common in Structural Engineering that is important for understanding the approach and methods described in this study. In addition, near-field and far-field and the cause of distortion during data acquisition will be discussed. Then, some relevant studies about vibration-based-localization methods are reviewed; their advantages, limitations, and how they can be applied for this study with corresponding adjustments are discussed.

\subsubsection{Floor Vibration Overview - Source, Path, and Receiver}

Floor vibration measurements have been used in buildings for many different applications, such as experimental modal analysis to determine natural frequencies, mode shapes, and damping ratios. Such measurements are also used to assess the vibration serviceability of a floor relative to human activity or mechanical machinery installed inside or near the building. The floor serviceability and vibration level acceptability depend on the following three main elements that have significant variability and randomness (Pavic and Zivanovic 2007):

- Vibration source

- Vibration path

- Vibration receiver

The vibration source can induce a dynamic load on a floor and excite it. A common problematic scenario is machinery-induced vibrations causing excessive vibration of a floor. Thus, such sources of stationary vibrations are the target of this study.

In this study, the vibration path through which the vibration waves travel is a floor system. The main challenge in processing signals from received vibrational waves moving through a floor is the path-dependent changes they undergo - affected by details of the floor framing, non-structural components, and boundary conditions - before they are captured by the sensors. The signal processing techniques should compensate for or mitigate these effects. More details are included in Section 1.2.2.

The vibration receiver in a building is either a human occupant, or occupants, or a sensitive equipment item. For example, based on Chapter 2 of the American Institute of 
Steel Construction Design Guide 11 (Murray et al. 2016), the recommended tolerance limit for human comfort in offices, residences, and quiet areas is $0.5 \%$ of the acceleration of gravity or $0.5 \%$ g. As another example, based on Chapter 6 of Design Guide 11, the tolerance limit for an electron microscope up to 30,000X magnification is 500 micro inch per second or 500 mips. If the vibration level exceeds the tolerance limit, then it will be necessary to identify the vibration source.

\subsubsection{Vibration Path and Wave Propagation Basics}

Wave propagation is the transfer of energy from a mechanical disturbance that moves through a medium. The particles in the medium are displaced to create vibration as the wave travels through, but they do not travel with the wave. The best visual example of waves can be seen when a small stone strikes a water surface forming ripples that are circular because the waves propagate in all horizontal directions at the same speed. The situation is similar for sound waves propagating in the air. Since the air is an approximately homogenous and isotropic medium, the propagation speed is approximately the same in all directions. Although there are many common features between acoustic and vibration waves traveling through a solid medium, there are some important differences.

Acoustic waves transfer through gases by moving particles back and forth in the direction of propagation; these are known as compressional waves. The vibration waves can also travel through solid media in this manner, and the term of this type of vibration propagation in solids is primary waves, P-waves, or in-plane waves, see Figure 1.1. In addition, vibration waves can oscillate perpendicular to the propagation direction, which is known as a secondary wave, S-wave, or out-of-plane wave, to carry energy away through the solid media (Worden 2001), see Figure 1.1. It should be mentioned that Swaves can travel only through solid materials because only solid materials can resist shear stress and, therefore, cause motion in the direction perpendicular to the direction of propagation, such as in the vertical direction in a floor. S-waves cannot propagate in gases or liquids with zero or very low viscosity (Sato et al. 2012). S-waves carry more energy than P-waves. This is why they are more likely to be felt by humans and why S-waves are often more destructive than P-waves in an earthquake. 
Furthermore, the properties of the waves traveling along the surfaces of solid media may be different, depending on the elasticity and thickness of the medium (Farnel 1970). There are different types of surface waves, and their explanations are beyond the scope of this study. According to Mirshekari et al. (2018), the vibration waves that travel through building floors with free boundaries at the top and bottom are known as Lamb waves. Similar to S-waves, particles in Lamb waves move perpendicular to the propagation direction but in a rolling motion in the plane along with the propagation. Note that Figure 1.1 shows Lamb waves with free boundaries only at the top. The properties of lamb waves are complex (Lamb 1881; Viktorov 1967). For the sake of simplicity, these vibration waves will be called vertical vibrations in this dissertation. The human body and some equipment items, like MRI, microscopes, and cameras, are more sensitive to floor vertical accelerations. Hence, the seismic sensors used in this research measure acceleration data only in Z-direction.

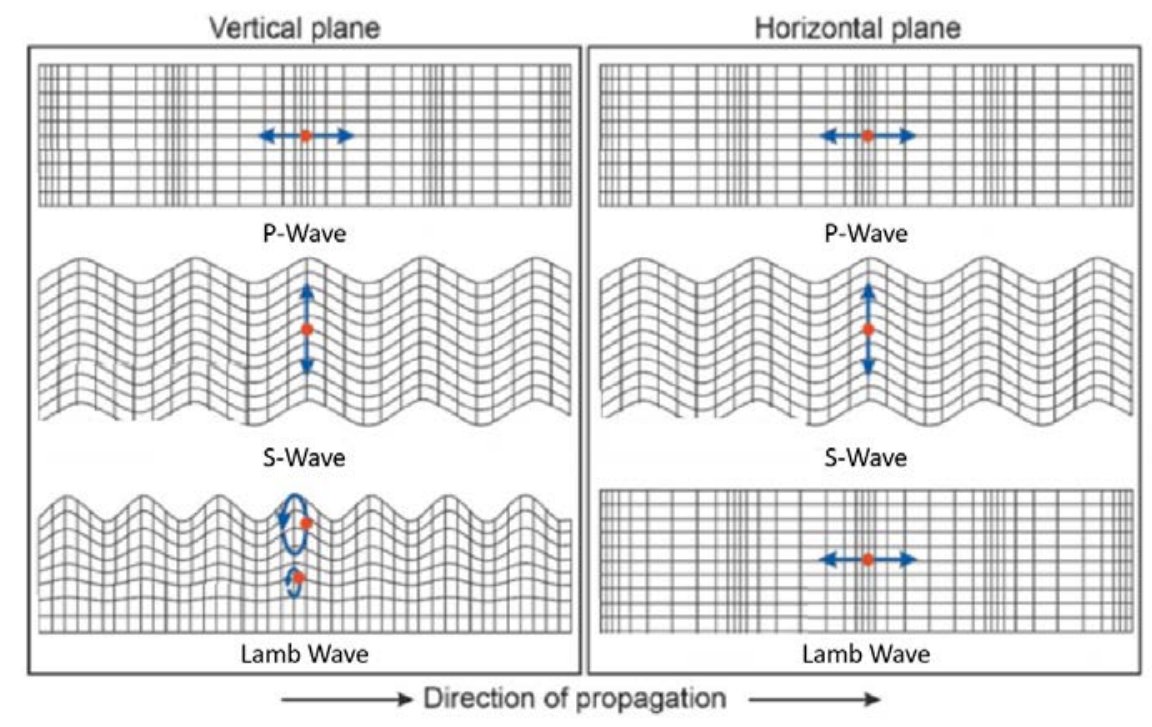

Figure 1.1: P-Wave, S-Wave, and Lamb Wave in Vertical and Horizontal Plane (Photo by Dondurur 2018 ${ }^{1}$ )

In a homogeneous solid media, such as a steel plate, the waves propagate in a more predictable manner. However, the wave propagation is more complicated for a typical floor with concrete slab, beams, and girders, which cannot be considered a homogenous

\footnotetext{
${ }^{1}$ Permission was granted to use this figure. This book was published in ELSEVIER, Author: Derma Dondurur, Title: Acquisition and Processing of Marine Seismic Data, Year: 2018.
} 
and isotropic material (Philippidis and Aggelis 2005). Also, the boundary conditions of a concrete floor (e.g., slab edges and openings), columns, girders, beams, degree of composite, and reinforcement, adds more complexity. Therefore, the processing of vibration waves traveling through the concrete floor is very complex. Analysis of waves in a concrete floor system has many applications (Worden 2001; Lee et al. 2016).

\subsubsection{Near Field vs. Far Field Region}

The distance between the vibration source and receiver significantly affects the waves that reach the receiver. If the receiver is far from the source, that is in a far-field region, and the radius of the wave is large, and the shape of the waves can be considered

planar and parallel to each other with no curvature, see Figure 1.2. This is the case in seismology when the distance between the epicenter and each seismograph is quite large, e.g., on the order of hundreds of kilometers; therefore, the direction of the earthquake waves is observable by processing seismic data. However, in a near-field region, such as inside a building, the vibration waves behave more complicated and unpredictable. When the vibration waves are close to the sensors, the shape of the waves are curved, and the curvature level depends on source-sensor distance. Therefore, received signals may vary from sensor to sensor, depending on how far they are from the source (Chen et al. 2002). The energy of waves decreases more quickly in near-field than far-field regions. Not only are the types of sensors deployed for the near-field region different from those used in farfield regions, but the data collected from a sensor in the near-field also requires more complicated techniques to be post-processed to obtain location information (Richman et al. 2001). This makes the localization of the vibration source using floor vibration measurements a challenging task. It will be explained in Section 1.3 why the localization method presented in this dissertation is more suitable for near-field applications. The waves shown in Figure 1.2 are in Z-direction. 


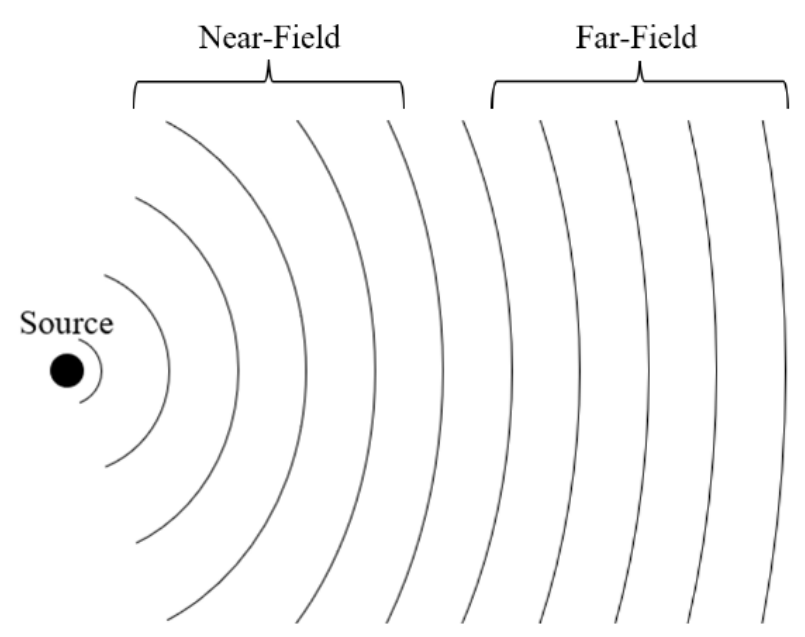

Figure 1.2: Near-Field Versus Far-Field

Before further discussions about near-field and far-field regions and their applications in this study, the terms "frequency" and "wavelength" need to be defined. Frequency is the number of oscillations in a unit of time, while wavelength is the distance traveled by one cycle. The relationship between wavelength, frequency, and propagation speed, is shown in Equation 1.1:

$$
\lambda=\frac{\text { Speed }}{\text { Frequency }}
$$

where $\lambda$ is the wavelength. This equation states that, for a constant wavelength, the frequency and speed are directly proportional to each other. The equation can be applied for a waveform with a single frequency. However, waves consist of components with different frequencies, phases, and amplitudes. For such complex waves, Equation 1.1 is not valid. Another challenge in the use of Equation 1.1 in this study is that the wave propagation speed in a concrete floor system is unknown. Section 1.2.4 explains how frequency-dependency of wave propagation causes challenges in processing vibration data that has traveled through a concrete floor.

As a practical example, in the design of ground-borne vibration barriers, there are two types of vibration isolation systems: near-field and far-field. Therefore, a proper boundary recognition between near-field and far-field regions is necessary and depends on the type of vibration source and soil properties (Gao et al. 2014; Khan et al. 2019). Multiple studies present criteria to determine the near-field and far-field boundary based on the 
wavelength, e.g., Lysmer et al. (1966), Haupt (1981), and Woods (1968) reported 2.5 $\lambda$, $\lambda$,

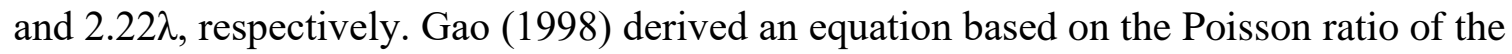
soil for the distinction between near-field and far-field boundaries. However, neither wavelength nor Poisson ratio is known for the floors that are the subject of this dissertation.

\subsubsection{Noise and Distortion Effects}

There are some terms used in this dissertation that are most common in the processing of wireless and audio signals. Since they are not commonly used in Structural Engineering, a brief description will be provided in this section with some tangible examples, and then the correlation of these concepts to this study will be explained.

A signal contains meaningful information. However, the term "noise" refers to unwanted variation that can interfere with the signal and modify its shape. It will be discussed in this section that how noise can be added to the signal during data acquisition.

\subsubsection{Multipath Propagation Effect}

A sound signal can reflect off boundaries, e.g., walls and ceilings, which means in addition to the direct path, sound waves also travel longer distances from secondary reflections. Therefore, the reflected sound signal is a weaker, distorted, and delayed version of the original sound signal. This phenomenon is known as a "reflection," and the best example is the time when someone can hear the reflected shouts in an open space such as a canyon. There is another term known as "reverberation," which is similar to reflection, but the time delay between original and reflected sounds is very short and usually occurs in a closed-space environment, such as when someone talks in an empty room with no carpet and furniture. Reverberation is considered "correlated noise” since it has many properties of the original signal but creates ambiguity in time delay estimation. Researchers in acoustics have proposed various methods to reduce the impact of reverberation when processing audio signals. The same challenge exists for vibration signals, particularly in indoor environments like elevated floors, and is known as the “multipath propagation effect," where the receivers acquire attenuated and delayed 
replicas of the source signal reflected from the boundaries - i.e., indirect path - in addition to the shortest and most direct path (Benesty et al. 2004). Indeed, the recorded vibration signals in an indoor environment are typically a mixture of direct and reflected waves (Bahroun et al. 2014) in the presence of obstacles like masonry or concrete walls, openings, columns, beams, or girders; this must be incorporated into the data postprocessing methodology. It should be noted that the speed of propagation in solid media, like a concrete floor, is much faster than the speed of waves in the air. Also, the effect of reflection is more probable in the near-field region. Therefore, there is more potential to have reflection during data acquisition in solid media.

In addition to the correlated noise, "random noise" or "uncorrelated noise" is captured during data acquisition. The sources of such noise can be either inside the building (Shi and Ming, 2016), such as a human walking, an elevator, mechanical equipment hung from beneath the floor, or a door slamming, or outside the building transferred into the building through the foundation, such as a moving train, moving trucks, or a close construction site.

\subsubsection{Attenuation}

The sound energy transferred into the air to vibrate molecules dissipates with distance traveled due to distribution in three dimensions (causing the intensity of the vibration in the air to drop) and the viscosity of the air. This can be referred to as "attenuation," which is a gradual loss of energy during propagation (Worden 2001). In a concrete floor system, friction causes the wave magnitude to drop over distance. Attenuation can be a combination of two factors: geometric damping and material damping. Geometric damping is a reduction of vibration-energy-density with distance, and it depends on the load source (e.g., line-load or point-load). Material damping depends on concrete floor properties and the frequency of vibration (Woods and Jedele 1985; Kim and

Lee 2000). Furthermore, nonstructural elements, such as ceilings can also affect the attenuation in a floor system. 


\subsubsection{Dispersion}

Wave is a combination of several components, each of which has a frequency, phase, and amplitude. If all components of the vibration wave travel with the same velocity, the phase and shape of the resultant wave are preserved along the propagation path. However, if wave components travel at different velocities, the sum of wave components does not maintain a consistent shape over time. This phenomenon is the case for many solid media, including floor systems, and is known as “dispersion.” A medium through which wave components with different frequencies travel at different speeds is called “dispersive” (Worden 2001; Mirshekari et al. 2016; Mirshekari et al. 2018). Studies have shown that attenuation is also frequency-dependent, which means the components have different attenuation levels (Kim and Lee 2000; Philippidis and Aggelis 2005). Indeed, as mentioned previously, material damping is a function of vibration frequency. Figure 1.3, which was also shown in Bahroun et al. (2014) and based on a simulation, illustrates the signals received by sensors distributed at different distances in a dispersive medium. In a non-dispersive medium, the shape of the wave does not change over time. However, in a dispersive medium, that different frequency travel at different speeds, the shape of wave is distorted. 


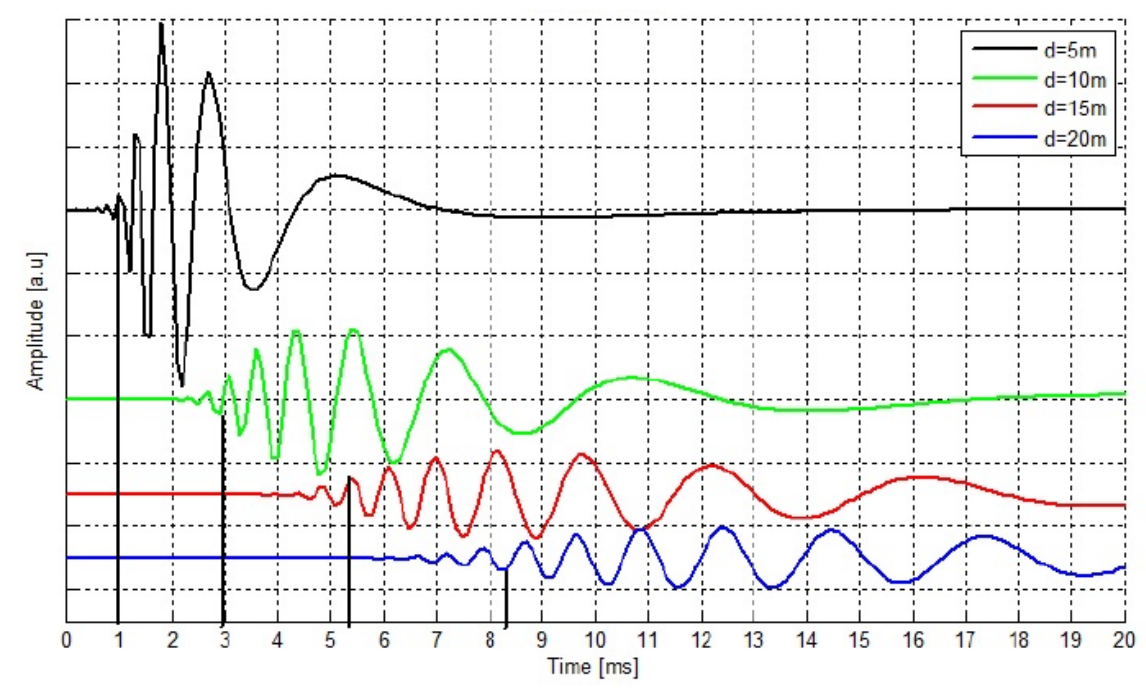

Figure 1.3: Effect of Dispersion on Simulated received Signals at Distances 5, 10, 15, and $20 \mathrm{~m}$ (Photo by Bahroun et al. 2014²)

\subsubsection{Localization Methods}

The identification of an earthquake epicenter is a well-known application of localization of vibration sources using seismic data (Geiger 1912; Kennett et al. 1995; Schweitzer 2001; Havskov et al. 2009). In seismology, seismographs record the timedomain waveform of the seismic waves. When several seismographs at different locations collect such waveforms, the time difference of arrival between P-waves and S-waves is estimated for each seismograph. With the use of specific charts, the distance between each seismograph and epicenter is estimated in the form of a circle around each seismograph location as possible source locations. The intersection of these circles is the likely epicenter, see Figure 1.4. Since the distance between the epicenter and each seismograph is quite large, e.g., on the order of hundreds of kilometers, the waves are planar and parallel, and, therefore, the direction of arrival is observable. As mentioned in Section 1.2.3, this is known as a far-field region.

\footnotetext{
2 Permission was granted to use this figure. This article was published in Journal of Sound and Vibration (ELSEVIER), Vol: 333(3), Authors: Bahroun, R., Michel, O., Frassati, F., Carmona, M. and Lacoume, J.L., Page Nos: 1046-1066, Title: New algorithm for footstep localization using seismic sensors in an indoor environment, Year: 2014.
} 


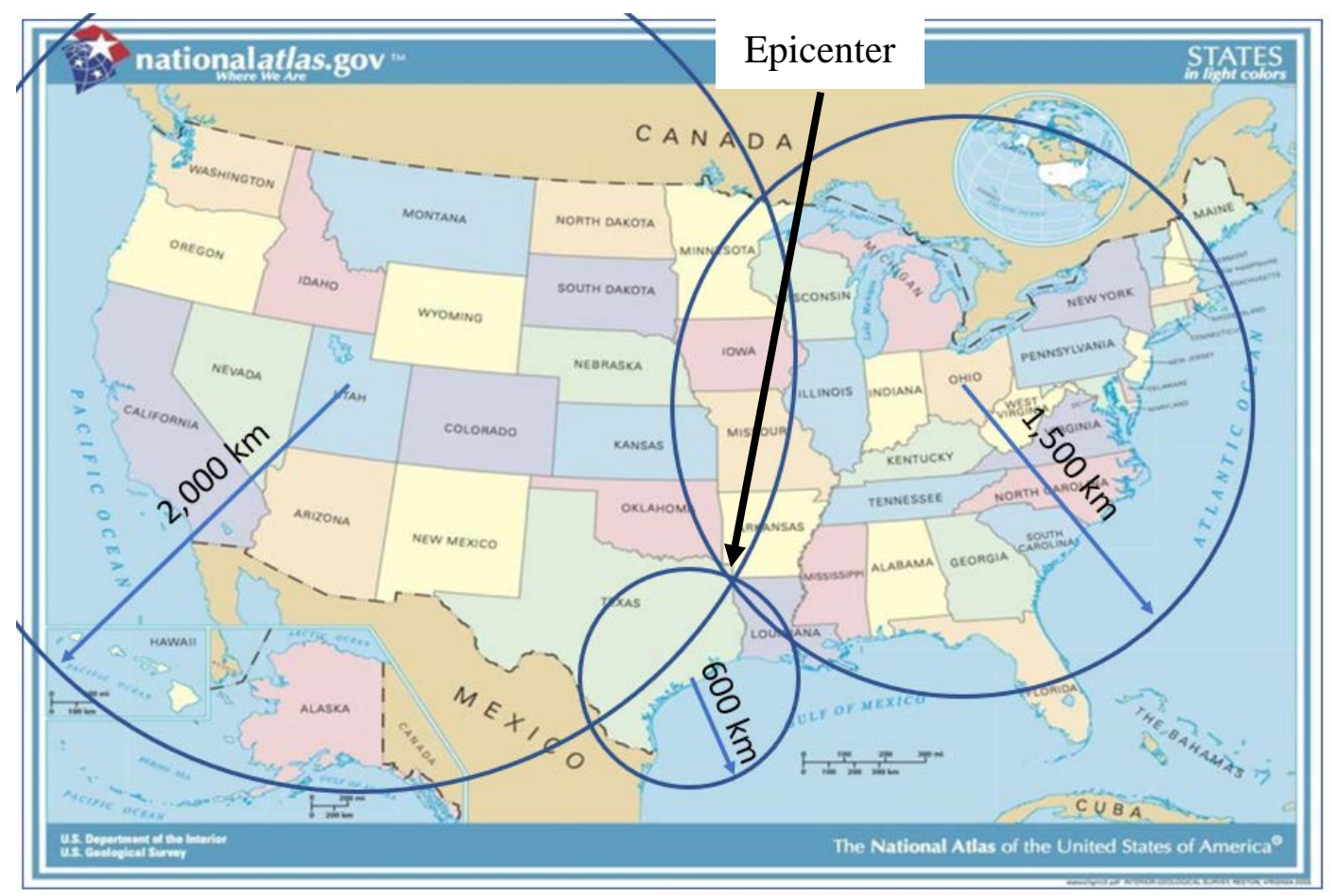

Figure 1.4: Localization of Epicenter (Credit: National Atlas, U.S. Geological Survey. Public domain.)

However, the epicenter-localization approach described above does not apply to near-field applications such as inside a building. In near-field applications, the shape of the received signal depends on the distance between each sensor and the source (Chen et al. 2002). This makes the localization of the vibration source using floor vibration measurements a challenging task.

Vibration sensors in buildings have been used for structural health monitoring and evaluating vibrations relative to tolerance limits. Potential uses in smart buildings are occupant tracking and identification of walking paths due to footstep-induced floor vibration. Video cameras, wearable devices, motion-detector sensors, IR sensors, and RF sensors can be employed for these purposes. However, they have some limitations, such as privacy issues, costly installation, maintenance, and calibration. They also have some other limitations in indoor applications, such as clear line-of-sight, angle coverage, and multipath problem (Richman et al. 2001; Bahroun et al. 2014; Pan et al. 2014; Shi and Ming 2016; Lam et al. 2016). 
The motivation behind the vibration-sensing localization approaches is that they are a privacy-respecting solution, low cost, and easy to be installed and maintained. Furthermore, these approaches have the potential to work well with sparse sensors (Pan et al. 2017; Drira et al. 2019; Mirshekari et al. 2018; Davis et al. 2021). Despite such advantages, indoor vibration-sensing-localization approaches still have the multipath effect challenge. In most cases, the existing methods for footstep localization are effective only under limited conditions - e.g., single walker, straight walking path, constant stride length, and walking continuously without stopping or reversing direction. These vibrationsensing localization approaches inspired portions of the research described herein, but they must be significantly modified to be applicable to the localization of stationary vibration sources.

Some localization approaches that are potentially effective for sources in buildings are described in the following paragraphs. One of the low-cost, easily implementable, and yet not commonly used to locate the source of vibration is Received Signal Strength or RSS (Lee et al. 2009; Bahroun et al. 2014, Niu et al. 2018). In this approach, prior to localization, the energy-loss versus distance model, i.e., the attenuation pattern, must be established; following this, the processing of data received by a group of sensors provides a set of candidate locations in the form of a circle for each sensor. The intersection of circles is the most likely location of the source of vibration. See Figure 1.5. In this figure, R1, R2, and R3 stand for receiver one, receiver two, or receiver three. 


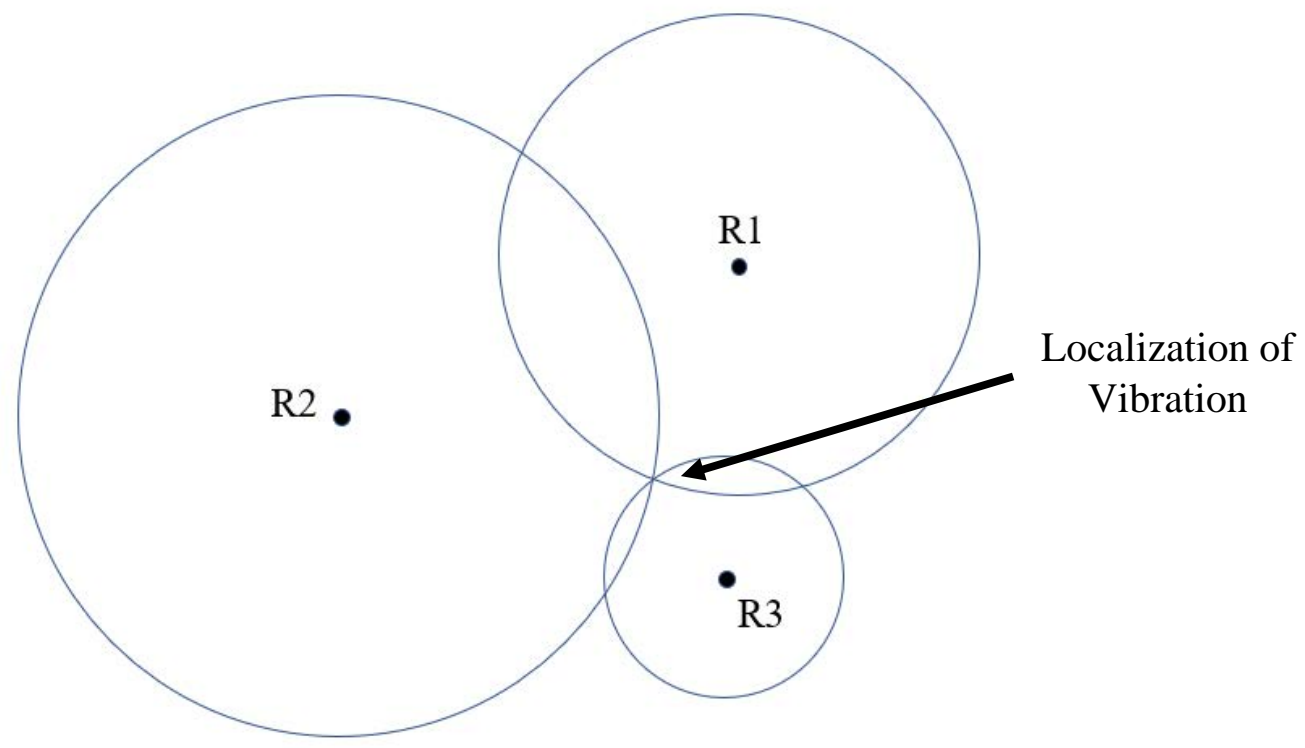

Figure 1.5: Vibration Source Localization Using RSS Method

It is assumed in this approach that the energy of propagated vibration decreases as the distance between source and sensor increases so that a sensor farther from the source is expected to measure a lower amplitude than a sensor closer to it. This is a good approximation for sound and wireless signals in the absence of boundaries. However, this assumption may not be valid for a concrete floor. For the beam example shown in Figure 1.6 with three pinned supports, the source exerts a vertical dynamic force and excites the first mode of vertical vibration. The red lines indicate the shape of the motion of the beam, corresponding to the first mode shape. Sensor 1 is nearer the source, and Sensor 2 is farther from the source. However, Sensor 1 is at a location with a lower mode shape amplitude. Thus, the response at Sensor 2 might be higher than the response at Sensor 1.

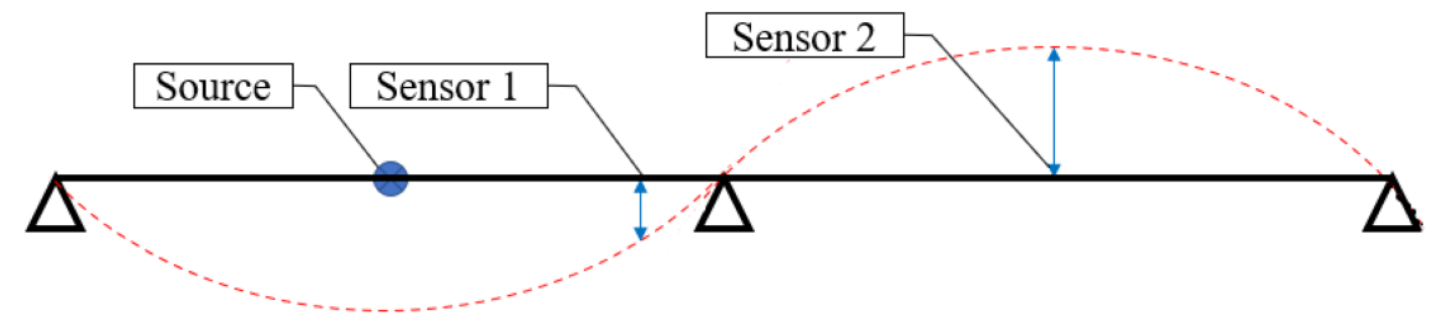

Figure 1.6: Source Excites the First Mode, Sensor 2 Reports a Higher Response 
Also, the attenuation pattern is unknown for real floors and needs to be modeled, which can be very unreliable (Mirshekari et al. 2018). However, in the case that such a pattern can be modeled, it must be modified for the material properties and boundary conditions of each bay.

Another established strategy of indoor localization using vibration measurements is based on the time difference of arrival (TDOA), which measures the differences in the arrival times of signals in a set of sensors (Torrieri 1984, Richman et al. 2001, Zheng et al. 2007, Bahroun et al. 2014, O’Keefe 2017). All TDOA-based localization algorithms are based on the assumption that wave propagation speed is known and constant in all directions. Consider the set of three sensors in a flat plane shown in Figure 1.7. Based on the time delay between a pair of sensors such as S1 and S2, the algorithm identifies a group of points for potential locations of the source that might have generated that delay. The delays between S1 and S3, and S2 and S3 are also used to identify a group of points. One of the TDOA localization-based approaches is the hyperbolic algorithm that results from the time difference estimate for each pair of sensors and provides a set of possible locations by solving nonlinear equations in two-dimensional space (Chan and Ho 1994). The intersection of two or more hyperbolas is generally considered the most likely source location. 


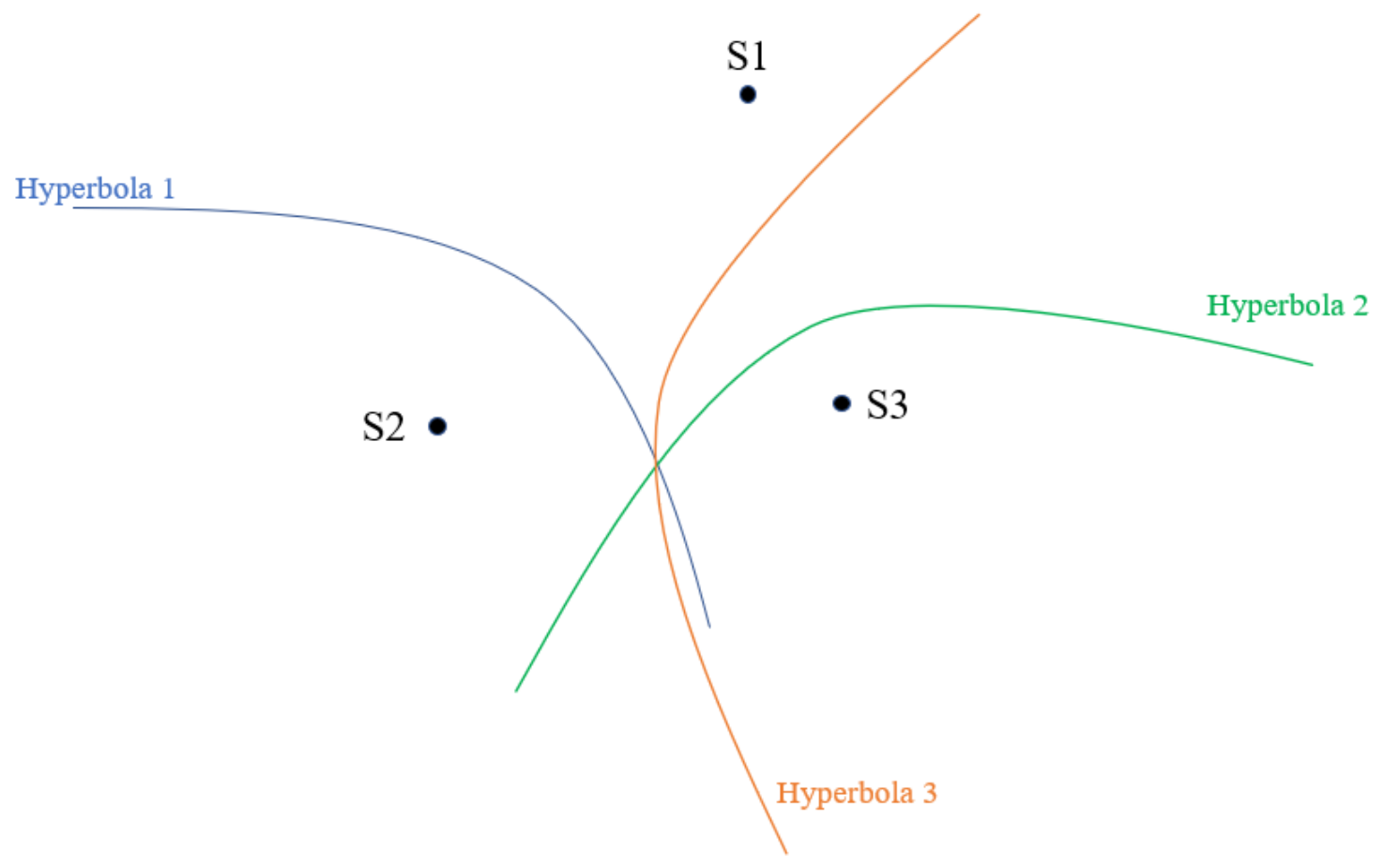

Figure 1.7: Vibration Source Localization Using TDOA Algorithm

This TDOA approach has been well studied and employed for in-air propagation applications - e.g., sound waves - in three-dimensional space since propagation speed is measurable and approximately constant in all directions. In this case, the accuracy of sound source localization using the TDOA approach depends on the correct estimation of parameters of which the speed of sound is a function, e.g., humidity and temperature (DiBiase et al. 2001). However, the situation is more challenging for solid media since the wave propagation speed is a function of more parameters with higher complexity. The level of variabilities for a concrete floor is notably higher, and there is no simple model for the estimation of the mechanical properties and boundary conditions on which the vibration propagation speed is based (Chen et al. 2002). Research has shown that wave propagation speed is highly variable in concrete floors. Richman et al. (2001) estimated wave propagation speed of $168 \mathrm{~m} / \mathrm{s}$ and an error of $8.4 \mathrm{~m}$ for footstep localization in a 40 m walking path. In another study, Schloemann et al. (2015) deployed a TDOA-based technique to determine the hammer impact location in a corridor of a smart building on 38 $\mathrm{m}$ by $35 \mathrm{~m}$ concrete floors with errors ranging from $1.0 \mathrm{~m}$ to $3.0 \mathrm{~m}$. The wave propagation speed was assumed at $914 \mathrm{~m} / \mathrm{s}$, but they did not describe how they determined this value. 
Moreover, Lee et al. (2009) mentioned that footstep vibration propagation speed exceeded $1500 \mathrm{~m} / \mathrm{s}$ in their study. As illustrated by these examples, the variation in wave propagation speed is relatively wide, perhaps because the speed is a function of many unmeasurable parameters.

Bahroun et al. (2014) developed a new, fast, and computationally more efficient TDOA-based algorithm to locate the vibration source on a concrete floor by identifying the order of arrival of vibration data among different sensors without using the computation of wave propagation speed. Their method is limited to intermittent vibration events, like walking, under low ambient noise, so it does not apply directly in the study presented in this dissertation. However, the computation of time of arrival in this method will be used in this dissertation, with the modification described in Chapter 2. Bahroun et al. also discussed in detail the difficulties in estimating wave propagation speed due to the effect of attenuation, dispersion, and reflection in the concrete floor. Despite these difficulties, the estimation of wave propagation speed is still needed herein.

Mirshekari et al. (2018) also used a TDOA-based algorithm with the addition of two signal processing techniques: wavelet decomposition (Addison, 2017) and adaptive multilateration approach to mitigate and deal with signal distortion due to dispersion and increase the accuracy of localization. His target was the localization of footstep-induced vibration. It was mentioned in this study that a vibration signal due to footstep falls into the category non-stationary signal, which means signal properties change over time. However, the target of this dissertation is the localization of machinery-induced vibration whose signal properties do not significantly change over time, which is known as a stationary signal. The effect of wavelet decomposition on the localization accuracy is one of future research that will be mentioned in Chapter 4.

Drira et al. (2019) developed a novel algorithm to consider dynamic characteristics of structure as well as the measurements to accommodate uncertainties - e.g., material properties and the boundary conditions - and improve the localization results of footstep localization using vibration measurements. They defined a set of possible locations and pre-recorded walking vibration measurements at each location to develop a prediction model. Their method is based on the detection and separation of each footstep vibration and assumes continuous walking without stopping or reversing directions with a constant 
stride length. To determine the walking path, each footstep vibration data is compared to the pre-defined prediction model on each possible location. In the case of a contradiction, the method rejects those locations to narrow down the possible true locations, which process is known as the falsification model in their study. This study cannot be used for the purpose of the research described herein for two reasons. First, their method is wellsuited for intermittent vibration events, while machinery-induced vibrations generate continuous vibration. Also, there is no pre-knowledge about the nature of machineryinduced vibration causing an issue and, therefore, a prediction model cannot be obtained.

Davis et al. (2021) used a reverse-engineering approach to detect and localize an impact, particularly a human fall since $50 \%$ or more injury-related deaths of 65 years or older are due to falls. Their method is based on the dynamics of the structure and the computation of the transfer function between a force and structural response, and it does not need to estimate the time of arrival and the time synchronization between sensors. Furthermore, this method detects not only the location of the impact, but also estimates the impact force. They reported very successful results in full-scale structural buildings. In this dissertation, time synchronization for all sensors is required for the estimation of time of arrivals.

The target of the studies mentioned above is mostly the localization of impulsive vibration events - e.g., a hammer strike, jumping, and particularly walking — with a broadband frequency range. Regarding walking footstep, although most of the energy of a single footstep excitation ranges in frequency from $0 \mathrm{~Hz}$ to $6 \mathrm{~Hz}$ (Ohlsson 1982), Brownjohn et al. (2004) empirically showed that, in addition to the frequency range of a single footstep, the real forcing function of walking includes its harmonic components i.e., the integer multiplication of step frequency. Therefore, the effective frequency range of walking is $1.9 \mathrm{~Hz}$ to $12 \mathrm{~Hz}$. More details regarding the nature of a walking footstep are beyond the scope of this study. The point is that the source of vibration in all aforementioned methods has a wider frequency range. However, stationary machinery usually induces single frequency vibration, which is the target of this study. Thus, the effect of distortion is less severe, which is an advantage in the research described herein. Nevertheless, the ideas of these methods were employed in the present study with some adjustments, as explained in Chapters 2 and 3. 
TDOA approaches seem to be a suitable choice for the localization of intermittent vibration events. However, for the application presented in this dissertation, TDOA may not be a good choice for the localization of machinery-induced vibration, which usually generates continuous vibration. Another problem with TDOA-based-localization is that noise acquired by pairs of sensors can result in ambiguity in the location of the vibration source. Nevertheless, a TDOA-based method will be used to estimate wave propagation speed, with some necessary modifications. A comprehensive estimation of wave propagation speed will be provided in Chapters 2 and 3.

\subsection{Research Objectives}

This dissertation aims to present a new floor vibration-based localization algorithm to locate a stationary machinery-induced vibration source on a concrete floor. This method, based on steered response power (SRP) method, is more robust than TDOA in the presence of structural variability - i.e., the non-uniform distribution of material properties and various boundary conditions in different bays - as well as attenuation, dispersion, and multipath effects, which are the main challenges in other indoor vibration-sensing localization approaches. Although theoretically, this algorithm can also be employed in other vibration-sensing localization applications, this dissertation is limited to the capability and sufficiency of this method to locate machinery-induced vibration.

The SRP algorithm has been used as a signal processing technique for wireless and acoustical applications to locate the source of wireless transmitter or speaker, respectively. However, this is the first study to apply the SRP method toward the localization of a vibration source. Generally, this method searches all candidate locations to find the most likely location of the vibration source. To compensate for direct path propagation delays, a time shift is applied to the vibration data regarding both the location of each receiver and the selected candidate location. Then, the shifted signals are summed, and the power associated with each candidate location is computed. The candidate location with the maximum power is the most likely location of the source of vibration. This algorithm is also known as the delay-and-sum procedure and will be further discussed in Chapters 2 and 3. Although the SRP method is computationally more demanding than TDOA-based localization, it is more robust in some applications (DiBiase et al. 2001). 
In addition, a prerequisite step to locating the vibration source using the SRP method is the estimation of wave propagation speed (WPS), and the accuracy of this localization depends on an accurate WPS estimation. Despite the complexity of the wave propagation in a concrete floor system, the estimation of WPS is possible since the material remains the same. Experiments can be performed to estimate WPS in the regions of interest. In TDOA, it is assumed that the WPS is identical in all directions, but this may not be accurate for concrete floors since they usually have different stiffnesses in the two orthogonal horizontal directions. A procedure to estimate WPS approximately in two perpendicular directions will be explained in Chapter 3. In addition, the TDOA performs a time delay estimation pairwise for all sensors, which is needed in subsequent steps. Therefore, any error associated with the time delay estimation affects the next steps negatively and reduces the localization accuracy of the TDOA algorithm. However, the SRP method neither estimates time delays nor processes the data in a pairwise sequence. Instead, it processes data from all sensors in each iteration to estimate steered power, which is more efficiently reduces noise effects. Another advantage of the SRP method over TDOA is that it can more easily account for different propagation speeds in multiple directions. For the processing of wireless signals and acoustical applications using the SRP method, WPS has been assumed constant in all direction. However, to the writer's knowledge, this study is the first to employ the SRP method to incorporate different speeds in various directions.

It is worth noting that the detection of a vibration event, e.g., footstep, hammer impact, falling object etc., using floor vibration measurements is not as complicated as localization, but it is a crucial step prior to vibration source localization (Mirshekari et al. 2016; Drira et al. 2019). A major challenge in vibration detection is the presence of ambient noise that might hide vibration event signatures. To overcome this limitation in this study, sensors with high sensitivity and high resolution have been used; more details will be provided in Chapter 3 .

Since this study is limited to the stationary vibration sources, the acceptable level of accuracy to estimate the vibration source would be expected an area with $3.0 \mathrm{~m}$ by 3.0 $\mathrm{m}$. In a consulting project, any clue about the location of the source would be a huge improvement over the current situation. Even with the use of a coarse estimate, an engineer 
can investigate and find the exact source. Even if an approach can identify the correct direction to the vibration source, it will be useful. 


\section{CHAPTER 2: STEERED RESPONSE POWER METHOD}

\subsection{Introduction}

This chapter aims to describe how the steered response power (SRP) method works, what parameters are needed prior to using this method, the challenges of using it. Also, the ways to enhance the SRP algorithm and make it more appropriate for the application presented herein are discussed.

In the SRP approach, a set of vibration sensors records the data from a concrete floor. The sensor locations are known. Also, a set of candidate source locations needs to be specified. This approach evaluates these locations by computing the propagation delay between each candidate source location and each vibration sensor location and then applying a specific time shift to the received signals. Next, the shifted signals are summed to compute the steered power associated with each candidate location. These steps are called the delay-and-sum procedure. At the source location, the shifted signals would align constructively, resulting in the maximum steered power. At other potential source locations, the shifted signals would have destructive alignment causing a lesser steered power. The candidate location with the maximum steered power is the most likely source location.

The process of locating the source of vibration given vibration measurements using multiple sensors has recently been of significant interest in Civil Engineering research, e.g., personnel tracking, structural vibration monitoring. These methods are becoming more accurate and practical. For example, structural vibration data has the potential to be post-processed to detect an underage walker and lock the liquor cabinet if he or she is close to the cabinet (Pan et al. 2017). The vibration-sensing approaches are privacy-respecting and easy to install and maintain, with the use of a sparse layout of vibration sensors. Although there are some alternatives to localization, e.g., camera, vision sensors, radio

frequency, and wearable devices (Budi et al. 2009; Zheng et al. 2011), they have limitations such as privacy issues, clear visual path, a high number of sensors, and installation. 
The base concept of the SRP method, beamforming, originated in the 1970s. Beamforming is a spatial filtering technique - mostly for wireless and acoustical applications - for steering the radiating signal in a specific direction using constructive interference while attenuating the radiation in other directions using destructive interference. This directional energy distribution, or directivity capability, is a more efficient signal radiation method than traditional and constant signal radiation in all directions. This idea of spatial filtering can be used for reception applications as well, such as radar, sonar, communications (Veen and Buckley 1988). The SRP method became the preferred technique for sound source localization applications because it is easily formulated and outperforms other methods against reverberation (Dmochowski et al. 2007)

It should be noted that the SRP method locates the vibration source correctly if both the vibration source and sensors are at the same elevated floor without any discontinuity (such as a big opening) in between the source and sensors (Dmochowski et al. 2007). This is because the vibration waves can be transferred from the source to the sensors directly through a single path, which is the concrete floor. However, the SRP method will not work if the vibration sensors are on the second floor and the source of vibration is on the third floor. In such a case, the vibration waves captured by the sensors have been transferred through multiple paths (i.e., through the upper floor slab to the columns and through the columns to the lower floor slab). As a further example, if the vibration sensors are on an elevated floor and the source of vibration is outside the building (e.g., a train, construction site), then the waves will be transferred through multiple paths (i.e., the ground, foundation, columns, and floor slab) to the floor on which the sensors are placed. In this case, as well, the SRP method will not work.

Regarding the discussion in Section 1.2.3, for the application presented in this dissertation and based on the empirical findings of the researcher, vibration propagation in the indoor building environment is assumed to be near-field based on the following reasons. The vibration level and corresponding energy attenuate rapidly with distance, which is one of the near-field propagation characteristics. Indeed, in a near-field region, each sensor receives a different gain due to both different propagation paths and various propagation speeds in different directions (Chen et al. 2002). However, in a far-field 
region, like an earthquake event, almost equal gain occurs for sensors placed within insignificant distance. It should be mentioned that the localization algorithm proposed in this dissertation is suitable for near-field region applications (Chen et al. 2001).

\subsection{Methodology}

This section describes the methodology of the SRP method. This method consists of two steps: the computation of time delay and then the estimation of steered power for all candidate source locations. The final localization decision can only be made when the computation of steered power for all candidate source locations has been computed. The type of sensor used in this dissertation to capture the vibration data from the concrete floor is the accelerometer. Therefore, the terms "sensor" and "accelerometer" are used interchangeably herein. It should be noted that in the entirety of this dissertation, the location of all accelerometers is known.

When the source generates a vibration signal, each accelerometer will receive the vibration response with a delay, $t$, related to the distance between the source and the accelerometer, as shown in Equation 2.1:

$$
A_{i}(t)=\alpha_{i} S\left(t+\tau_{i}\right)+k(t) \quad \text { Eq. } 2.1
$$

where $A_{i}(t)$ indicates the time-domain acceleration response for $i^{\text {th }}$ accelerometer due to the excitation signal generated by the source, $S(t), \alpha$ is an attenuation factor due to the propagation effects, $\tau_{i}$ is the time delay at the $i^{\text {th }}$ accelerometer, and $t$ represents the time since recording the waveform.

Indeed, the acceleration data acquired by $i^{\text {th }}$ accelerometer is the delayed and attenuated replica of the excitation signal. $k(t)$ is any possible noise, which can be independent noise, correlated noise, or a combination of both, as explained in Section 1.2.4.1.

The first step in signal processing is digitization, known as analog-to-digital conversion, to convert continuous-time data into a finite number of samples. Therefore, $t$ can be replaced with $n$, sample number, and Equation 2.1 can be re-written as:

$$
A_{i}(n)=\alpha_{i} S\left(n+n_{\tau_{i}}\right)+k(n)
$$




$$
n=F_{s} . t
$$

where $F_{s}$ is the sampling frequency in the unit of samples per second $(\mathrm{S} / \mathrm{sec}$ hereafter) and $n_{t i}$ is the time delay in the unit of samples.

In the SRP method, a set of candidate source locations is first defined. Each candidate location will be termed $L_{x, y}$, where $x, y$ represents the coordinates of the $x$-y plane spatial grid for each candidate location. The distance between the $i^{\text {th }}$ accelerometer and candidate location $L_{x, y}$ is:

$$
d_{A_{i}}=\sqrt{\left(x_{A_{i}}-x_{L_{x, y}}\right)^{2}+\left(y_{A_{i}}-y_{L_{x, y}}\right)^{2}}
$$

where $x_{A i}$ and $y_{A i}$ are the $\mathrm{x}$ and $\mathrm{y}$ coordinates of the $i^{\text {th }}$ accelerometer and $x_{L x, y}$ and $y_{L x, y}$ are the $\mathrm{x}$ and $\mathrm{y}$ coordinates of the candidate location.

Then, the time shift, $T S_{i}$, in units of time, between the candidate location and the $i^{\text {th }}$ accelerometer is:

$$
T S_{i}=\frac{d_{A_{i}}}{W P S}
$$

where WPS stands for the wave propagation speed. Estimation of WPS will be discussed in Section 2.3. The accuracy of $T S_{i}$ depends on the correct estimation of WPS.

Next, the $i^{\text {th }}$ time shift is applied to the signal from the $i^{\text {th }}$ accelerometer. It should be noted that $T S_{i}$ in Equation 2.5 has units of time, and its unit is converted to samples to be used in Equation 2.6:

$$
A_{i}\left(n-n_{T S_{i}}\right)=\alpha S\left(n+n_{\tau_{i}}-n_{T S_{i}}\right)+k\left(n-n_{T S_{i}}\right) \quad \text { Eq. } 2.6
$$

The purpose of shifting signals received by the accelerometers is to determine whether the delayed and attenuated replica of the excitation signal aligns constructively. Ideally, in the absence of noise (i.e., $k$ ), if $\tau_{i}$ and $T S_{i}$ are equal for all accelerometers, the shapes of the shifted versions of the received data among different accelerometers are identical except in the acceleration axis scale. The difference in scale is due to attenuation, represented by $\alpha$. If the shapes are identical, then the point-by-point addition will be additive, causing constructive interference. This means the candidate location is the actual source location.

Next, the steered power associated with the candidate location at $x, y$ is computed as shown in Equation 2.7: 


$$
Z_{x, y}=\sum_{n=1}^{N}\left(\sum_{i=1}^{m} A_{i}\left(n-n_{T S_{i}}\right)\right)^{2}
$$

where $Z_{x, y}$ is the steered power for each candidate location at the $x$ and $y$ coordinates, $m$ is the total number of accelerometers, and $N$ is the total number of time samples in each signal. It should be mentioned that $Z_{x, y}$ is the scalar for each candidate location (Royvaran et al. 2021). For simplicity, Figure 2.1 shows a schematic view of Equation 2.7.

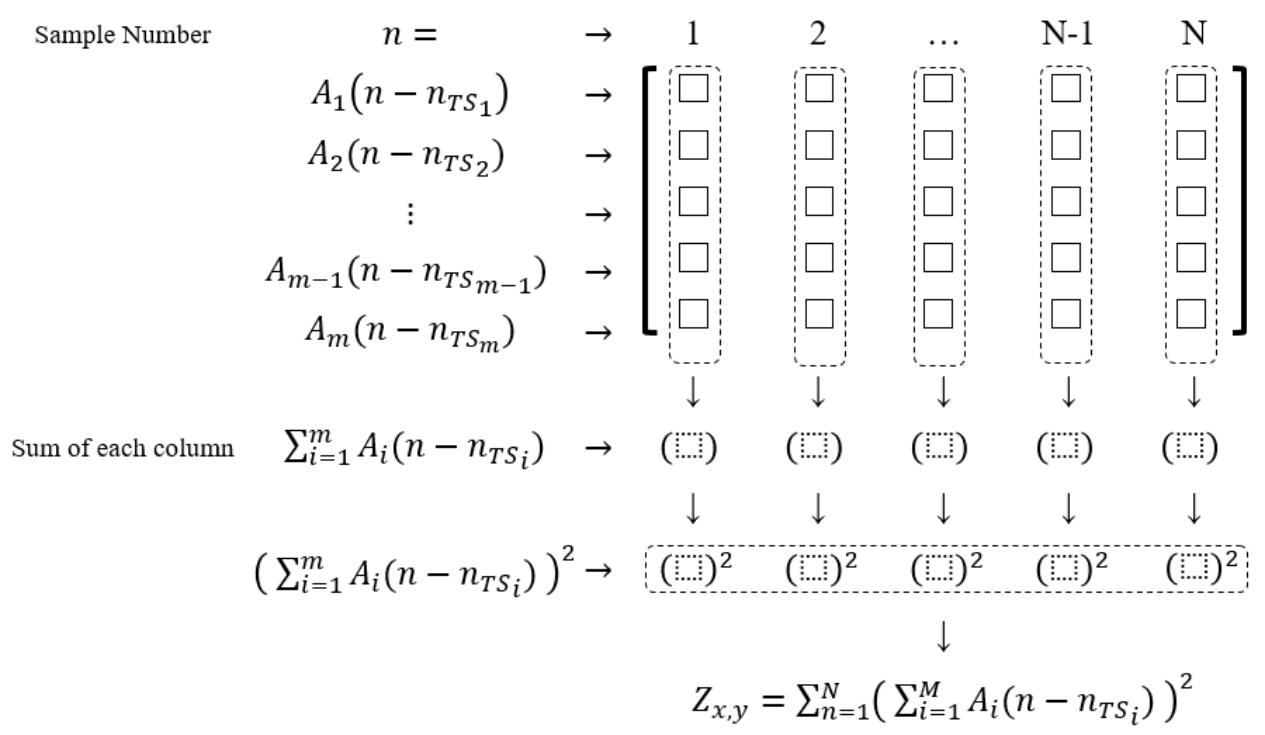

Figure 2.1: Schematic View of Equation 2.7

An idealized situation when constructive interference occurs at the real source of vibration was explained below Equation 2.6. In the real-world scenarios, due to some degrees of imprecision in the estimation of WPS and in the presence of noise, the shifted and attenuated versions of received accelerometers will not be exactly the same. Fortunately, SRP results are not binary with a "yes” or "no" declaration for each candidate location. Instead, the steered power is a numerical value that varies among the candidate locations; be maximum steered power is at the source location.

To better understand the concept of the SRP method and equations given above, the procedures are elaborated in the form of some simple visual simulations in this chapter. The list of simulations and their details are shown in Table 2.1. The effect of different parameters has been considered in this table. 
Table 2.1: List of Simulations and Their Details

\begin{tabular}{|c|c|c|c|c|c|c|c|c|c|c|c|c|c|}
\hline \multirow{3}{*}{ Simulation \# } & \multirow{2}{*}{ Fs } & \multirow{2}{*}{ WPS } & \multirow{2}{*}{$\begin{array}{c}\text { Grid } \\
\text { Resolution }\end{array}$} & \multirow{2}{*}{\begin{tabular}{c|c|} 
Time \\
Resolution
\end{tabular}} & \multirow{2}{*}{ Area } & \multirow{2}{*}{ Duration } & \multirow{2}{*}{$\begin{array}{c}\text { Source } \\
\text { Location }\end{array}$} & \multicolumn{4}{|c|}{ Coordinate } & \multirow{3}{*}{ SNR } & \multirow{3}{*}{$\begin{array}{c}\text { Source } \\
\text { Excitation }\end{array}$} \\
\hline & & & & & & & & A1 & A2 & A3 & A4 & & \\
\hline & S/s & $\mathbf{m} / \mathbf{s}$ & m & sec & m & sec & m & m & $\mathbf{m}$ & m & m & & \\
\hline 1 & 10 & 1 & 1 & 0.1 & $2 \times 2$ & 5 & $(0,1)$ & $(0,0)$ & $(2,0)$ & - & - & No Noise & Pulse \\
\hline 2 & 10 & 1 & 0.5 & 0.1 & $2 \mathrm{x} 2$ & 5 & $(0,1)$ & $(0,0)$ & $(2,0)$ & - & - & No Noise & Pulse \\
\hline 3 & 10 & 1 & 0.25 & 0.1 & $2 \mathrm{x} 2$ & 5 & $(0,1)$ & $(0,0)$ & $(2,0)$ & - & - & No Noise & Pulse \\
\hline 4 & 100 & 1 & 0.25 & 0.01 & $2 \times 2$ & 5 & $(0,1)$ & $(0,0)$ & $(2,0)$ & - & - & No Noise & Pulse \\
\hline 5 & 100 & 1 & 0.25 & 0.01 & $4 \times 4$ & 6 & $(1,2)$ & $(0,0)$ & $(4,0)$ & - & - & No Noise & ulse \\
\hline 6 & 100 & 1 & 0.25 & 0.01 & $4 \times 4$ & 6 & $(1,2)$ & $(0,0)$ & $(4,0)$ & - & - & 12.60 & Pulse \\
\hline 7 & 100 & 1 & 0.25 & 0.01 & $4 \times 4$ & 6 & $(1,2)$ & $(0,0)$ & $(4,0)$ & - & - & 2.15 & Pulse \\
\hline 8 & 100 & 1 & 0.25 & 0.01 & $4 \times 4$ & 6 & $(1,2)$ & $(0,0)$ & $(4,0)$ & - & - & .81 & Pulse \\
\hline 9 & 100 & 1 & 0.25 & 0.01 & $4 \times 4$ & 6 & $(2,1)$ & $(0,0)$ & $(4,0)$ & - & - & 2.51 & Pulse \\
\hline 10 & 100 & 1 & 0.25 & 0.01 & $4 \times 4$ & 6 & $(2,3)$ & $(0,0)$ & $(4,0)$ & - & - & 2.50 & Pulse \\
\hline 11 & 100 & 1 & 0.25 & 0.01 & $4 \times 4$ & 6 & $(2,1)$ & $(0,0)$ & $(3,1)$ & - & - & 2.49 & Pulse \\
\hline 12 & 1000 & 1 & 0.25 & 0.001 & $8 \times 8$ & 12 & $(2,3)$ & $(0,0)$ & $(8,0)$ & - & - & $>1000$ & Sin \\
\hline 13 & 1000 & 1 & 0.25 & 0.001 & $8 \times 8$ & 12 & $(2,3)$ & $(0,0)$ & $(8,0)$ & $(8,8)$ & - & $>1000$ & Sin \\
\hline 14 & 1000 & 1 & 0.25 & 0.001 & $8 \times 8$ & 12 & $(2,3)$ & $(0,0)$ & $(8,0)$ & $(8,8)$ & $(0,8)$ & $>1000$ & Sin \\
\hline 15 & 1000 & 1 & 0.25 & 0.001 & \begin{tabular}{|l|}
$8 \times 8$ \\
\end{tabular} & 12 & $(2,3)$ & $(8,0)$ & $(5,2)$ & \begin{tabular}{|l|}
$(8,2)$ \\
\end{tabular} & $(2,4)$ & $>1000$ & Sin \\
\hline 16 & 1000 & 1 & 0.25 & 0.001 & $8 \times 8$ & 12 & $(2,3)$ & $(8,0)$ & $(5,2)$ & $(8,2)$ & $(2,4)$ & $>1000$ & White \\
\hline 17 & 1000 & 10 & 0.25 & 0.001 & 10x10 & 2 & $(2,3)$ & $(2,1)$ & $(3,8)$ & $(10,9)$ & $(6,2)$ & $>300$ & White \\
\hline
\end{tabular}

In Simulation 1, the bay size is $2.0 \mathrm{~m}$ by $2.0 \mathrm{~m}$, and there are two accelerometers, $m=2$, as shown in Figure 2.2(a). The total duration of data acquisition is $5.0 \mathrm{sec}$, and the sampling frequency rate, $F_{s}$, is $10.0 \mathrm{~S} / \mathrm{sec}$. The number of samples in each signal is the duration multiplied by the sampling frequency rate. In this example, the WPS is $1.0 \mathrm{~m} / \mathrm{s}$, and it is constant in all directions. The effects of damping, attenuation, and multipath propagation are not considered in this simulation. The distance between the source and A1 and A2 accelerometers is $1.0 \mathrm{~m}$ and $2.2 \mathrm{~m}$, respectively. The location of the vibration source is at the $(0,1)$ meter coordinate, and the signal associated with the source is a single pulse with an amplitude of 2.0 that occurred at $t=2 \mathrm{sec}$. Since the propagation speed is 1.0 $\mathrm{m} / \mathrm{s}$, the generated impulse will be received by A1 and A2 accelerometers with 1.0 sec and 2.2 sec delays, respectively. See Figure 2.2(b). 


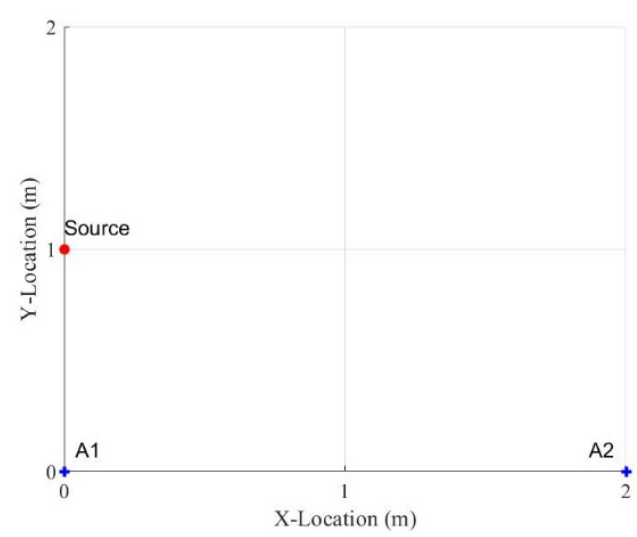

(a) Location of Source and Accelerometers
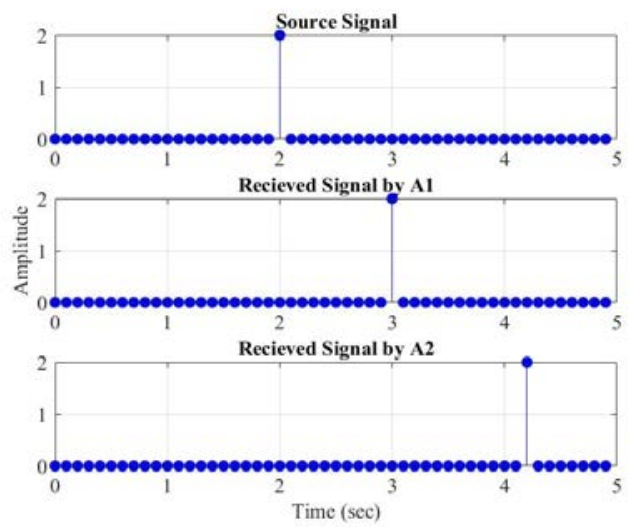

(b) Excitation and Received Signals

Figure 2.2: Simulation 1 Configuration and Excitation and Received Signals

To locate the vibration source using the SRP method, the accelerometer locations, the signals received by accelerometers, and WPS are parameters that are needed. The spatial grid resolution in this simulation is assumed to be $1.0 \mathrm{~m}$ by $1.0 \mathrm{~m}$. In Figure 2.3, the locations of two accelerometers are marked by blue "plus" symbols, and all candidate locations are marked by red circles. The accelerometer locations are excluded from the set of candidate locations as it is already known that the source is not at either of those locations.

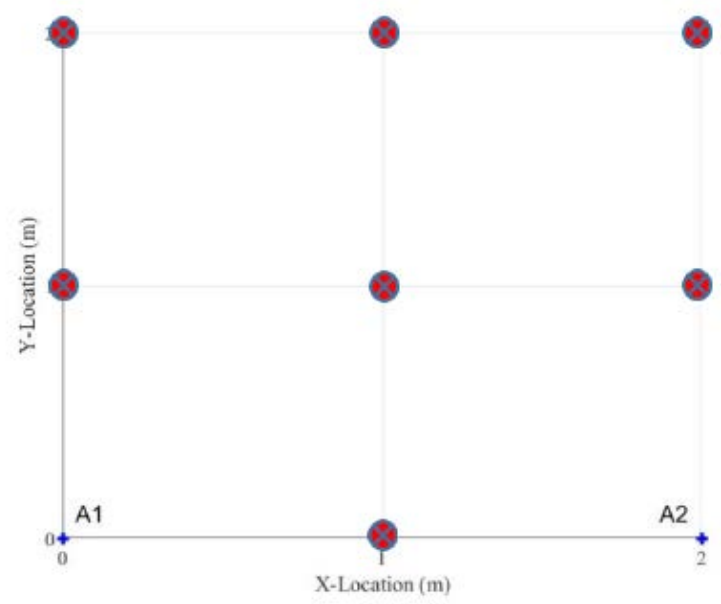

Figure 2.3: Candidate Locations as The Source of Vibration

In the SRP method, the steered power is computed for each candidate location to find the source of vibration. As illustrated in Figure 2.4(a), for each candidate location, the received signals by A1 and A2 accelerometers will be shifted — as shown by "shift A1" 
and "shift A2" - regarding their distances to each candidate location. These shifted signals correspond to Equation 2.6. For example, if the candidate location is at $(0,2)$ meter (Figure 2.4(a)), it has a distance of $2.0 \mathrm{~m}$ and $2.8 \mathrm{~m}$ from A1 and A2, respectively. Since the WPS is $1.0 \mathrm{~m} / \mathrm{s}$, the time shifts at $\mathrm{A} 1$ and $\mathrm{A} 2$ are $T S_{1}=2.0 \mathrm{sec}$ and $T S_{2}=2.8 \mathrm{sec}$. Then, the shifted versions of both signals are added together — as shown by "Sum=shift A1+shift A2” - and then each element in this vector will be raised to the power of two, as shown by "Sum". Summation of all elements in this resultant vector corresponds to Equation 2.7 and gives the power for this candidate location, as shown by " $Z_{0 m, 2 m}=\sum\left(\mathrm{Sum}^{2}\right)$ ". The steered power for this candidate location, $Z_{0 m, 2 m}=8.0$. In Figure 2.4(b), the candidate location at $(0,1)$ meter coordinate in this simulation is the correct estimate of the vibration source using the SRP method. At this location, the received impulse at the shifted versions of A1 and A2 accelerometers align (i.e., constructive interference), and the steered power is maximum. This process is also shown for two more candidate locations in Figures 2.4(c) and 2.4(d).

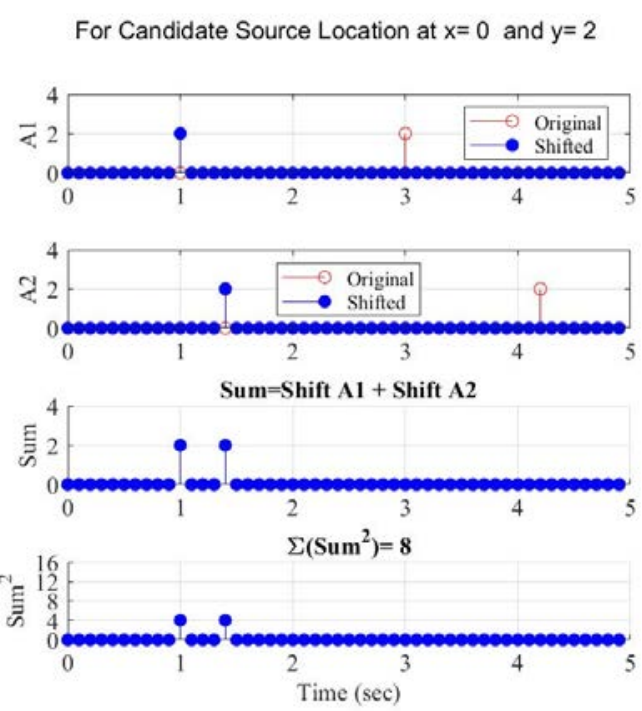

(a)

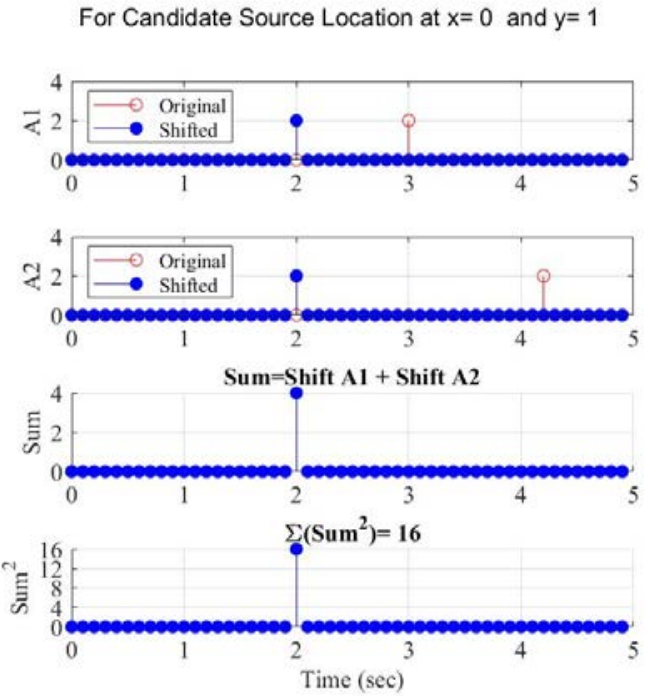

(b) 


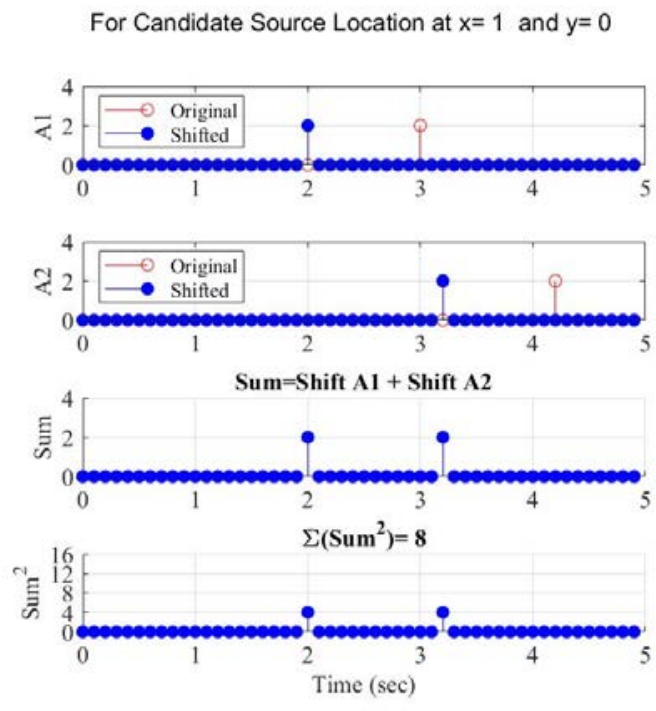

(c)

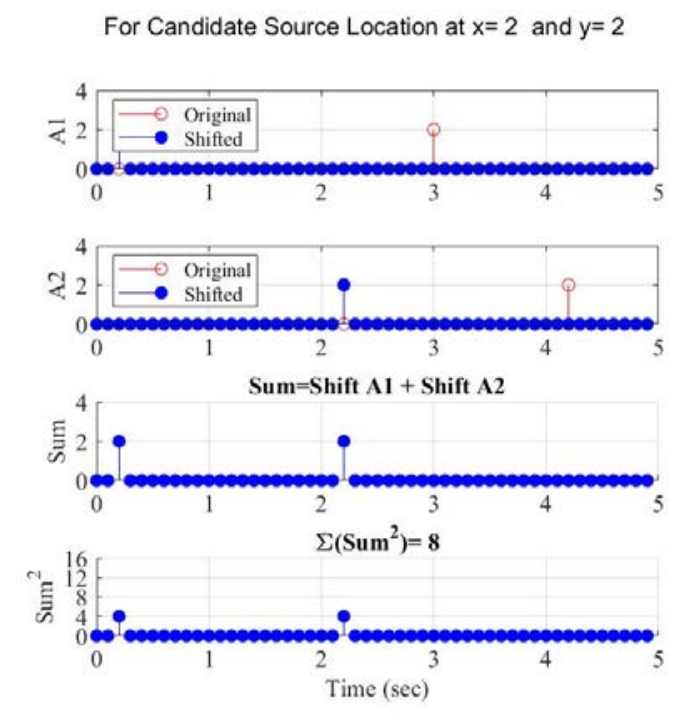

(d)

Figure 2.4: Visual Simulation of the SRP Method (Simulation 1)

The results for the steered power are shown in Figure 2.5. This figure can also be shown in the form of a 3D plot, as shown in Figure 2.6(a), and X-Y plane is the $2.0 \mathrm{~m}$ by $2.0 \mathrm{~m}$ simulation area, and the color bar on the right side expresses the steered power of the SRP method. The interpolated coloring in-between the grid points has been used based on the values corresponding to the grid points. However, the grid resolution of $1.0 \mathrm{~m}$ in this simulation is rather coarse. Figure 2.6(b) is the plan view of this 3D view plot, which is another representation of Figures 2.5 and 2.6(a). 


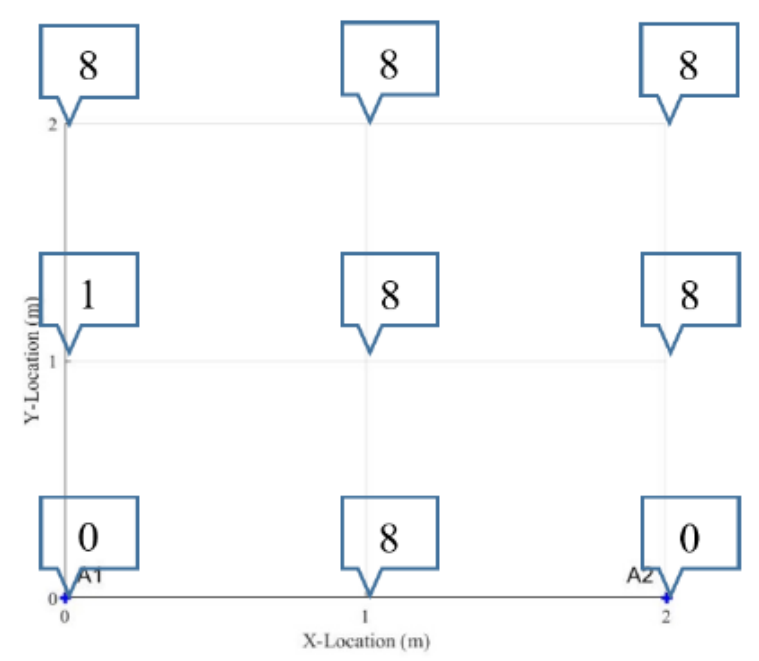

Figure 2.5: Results of Visual Simulation of the SRP Method (Simulation 1)

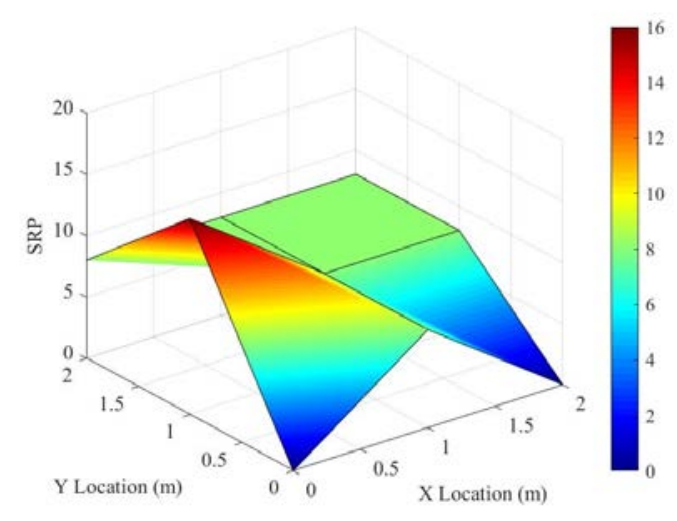

(a) 3D View

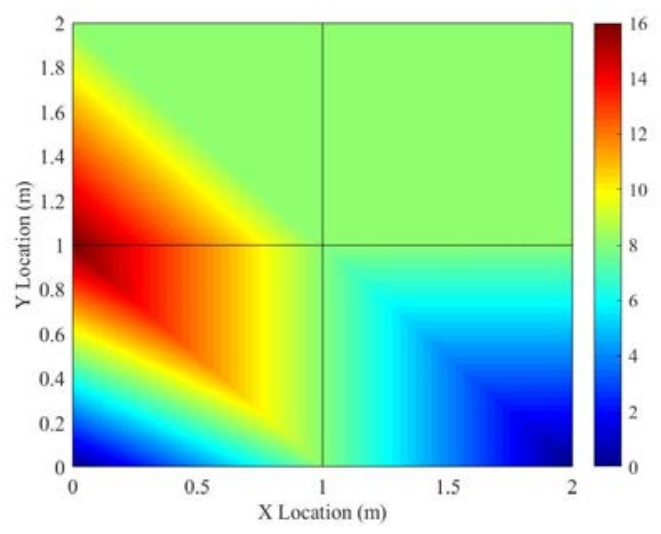

(b) Plan View

Figure 2.6: SRP Results for Simulation 1

The use of higher grid resolution makes the SRP plot smoother. However, by increasing the number of candidate locations, the number of locations to search increases, which comes with the cost of longer computation. Figure 2.7 shows steered power plots for the situation described above with grid resolutions of $0.5 \mathrm{~m}$ and $0.25 \mathrm{~m}$. These two simulations are referred to as Simulations 2 and 3, respectively. 


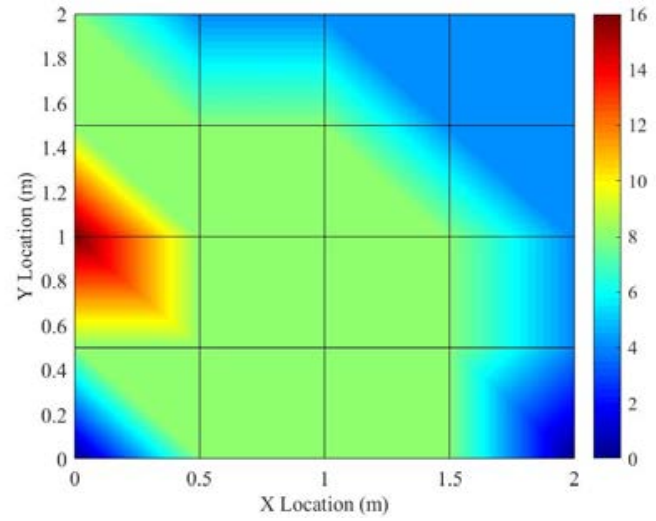

(a) Grid Resolution 0.5 m (Simulation 2)

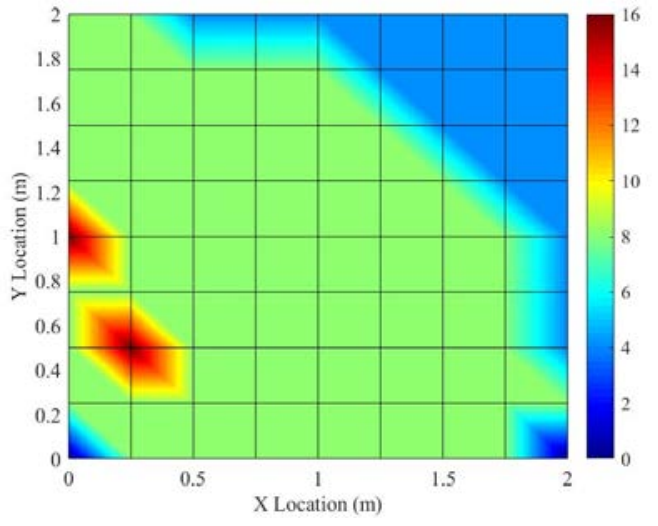

(b) Grid Resolution 0.25 m (Simulation 3)

Figure 2.7: Effect of Grid Resolution on The SRP Plot

Unexpectedly, in Figure 2.7(b), as well as the correct localization of the vibration source, another source location with steered power of 16 is shown at coordinates $(0.25,0.5)$ meters, which is incorrect. This is because of a round-off error due to the low sampling frequency in Simulation 3. At this point, A1 and A2 need to shift $0.55 \mathrm{sec}$ and $1.82 \mathrm{sec}$, but due to the time resolution of $0.1 \mathrm{sec}$ and the round-off error, the time shift changed to 0.6 and 1.8 seconds, leading to peaks at the shifted versions of A1 and A2 accelerometers to align undesirably. Figures 2.8(a) and 2.8(b) show the time shift at coordinate $(0.25,0.5)$ meters with a sampling frequency rate of 10 and $100 \mathrm{~S} / \mathrm{sec}$ for Simulations 3 and 4, respectively.

For Candidate Source Location at $\mathrm{x}=0.25$ and $\mathrm{y}=0.5$
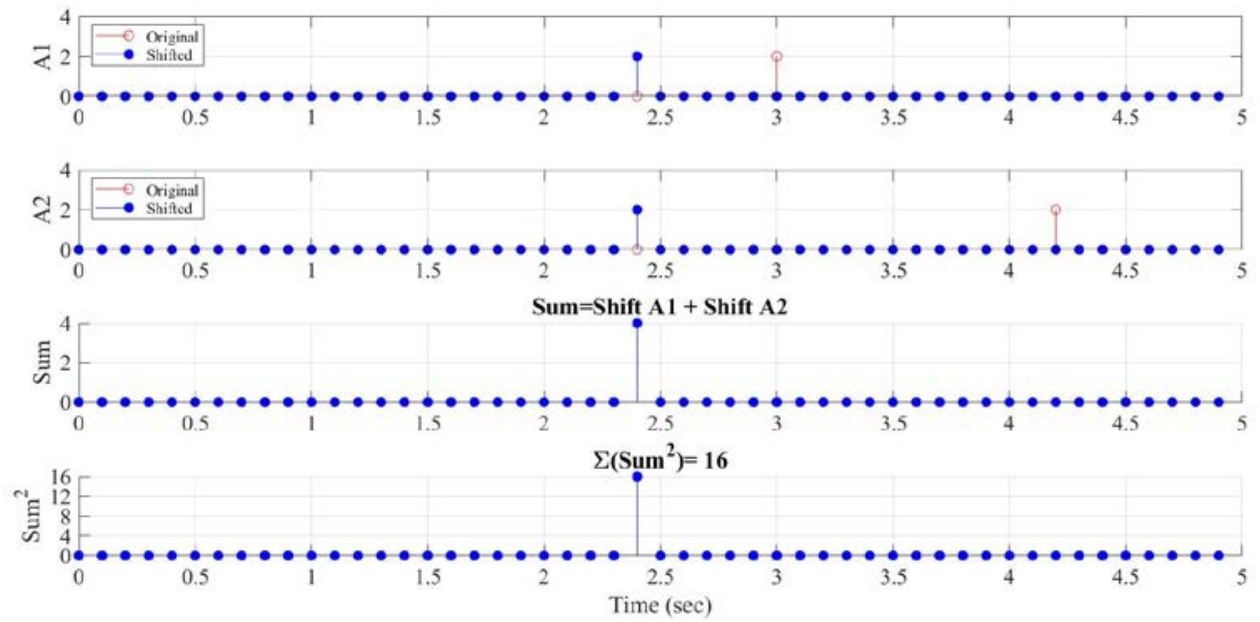

(a) $F_{s}=10 \mathrm{~S} / \mathrm{sec}($ Simulation 3) 
For Candidate Source Location at $\mathrm{x}=0.25$ and $\mathrm{y}=0.5$
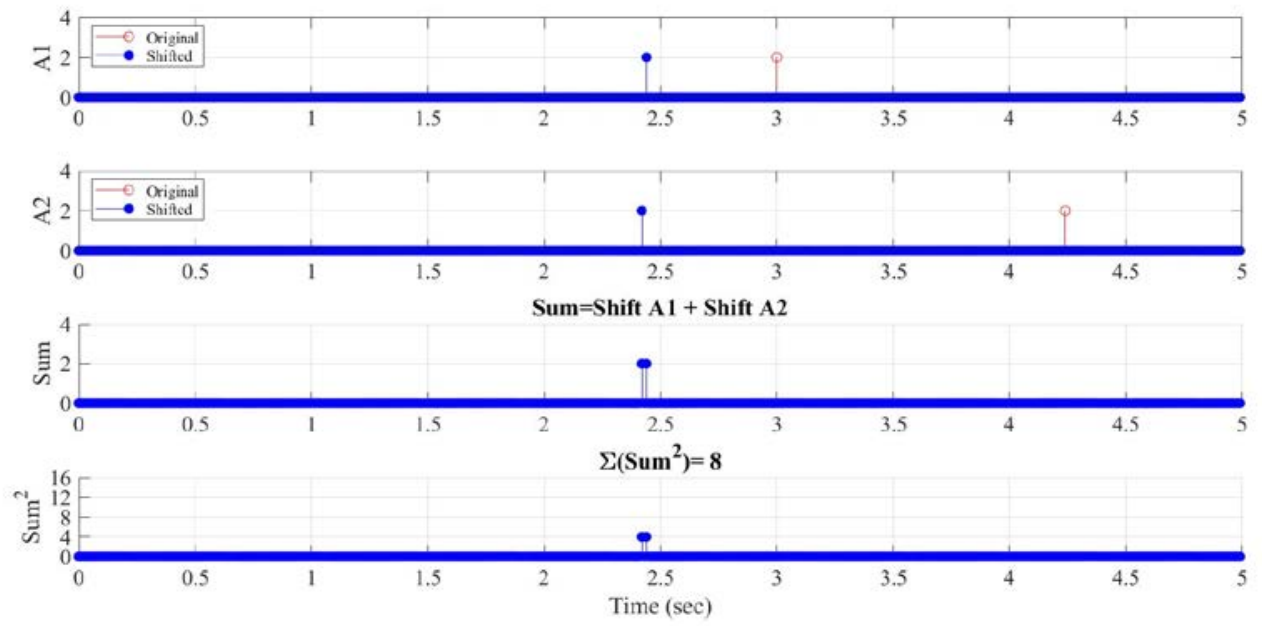

(b) $F_{s}=100 \mathrm{~S} / \mathrm{sec}$ (Simulation 4)

Figure 2.8: Effect of Sampling Frequency Rate on Time Shift

With the increasing of sampling frequency rate from $10 \mathrm{~S} / \mathrm{sec}$ in Simulation 3 (Figure 2.8(a)) to $100 \mathrm{~S} / \mathrm{sec}$ in Simulation 4 (Figure 2.8(b)), the incorrect steered power at coordinates $(0.25,0.5)$ meter dropped to eight. The time resolution between two consecutive blue dots in Figures 2.8(a) and 2.8(b) is $0.1 \mathrm{sec}$ and $0.01 \mathrm{sec}$, respectively. For comparison, the effect of sampling frequency on the SRP results is shown in Figure 2.9. List of simulations was shown in Table 2.1.

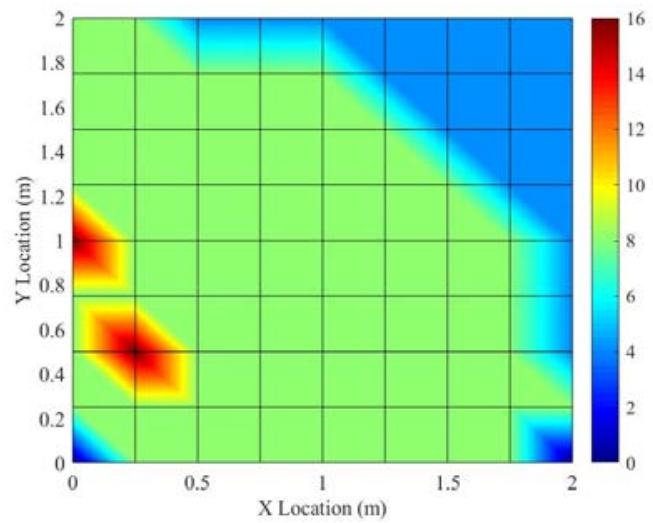

(a) $F_{s}=10 \mathrm{~S} / \mathrm{sec}($ Simulation 3$)$

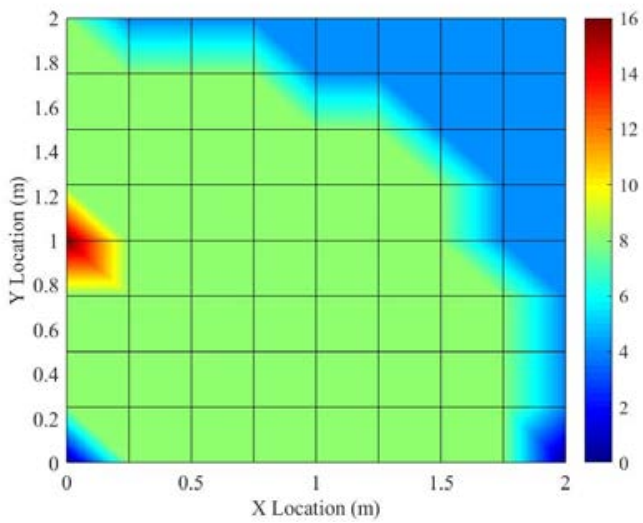

(b) $F_{s}=100 \mathrm{~S} / \mathrm{sec}($ Simulation 4$)$

Figure 2.9: Effect of Sampling Frequency on SRP Results 
Next, the effect of noise, denoted by $k$ in Equation 2.1, on the SRP algorithm will be described, but the term "power of signal" must be explained first. Power of signal, $P$, is the amount of energy per unit of time, and it can be calculated in an analog signal using Equation 2.8:

$$
P=\lim _{T \rightarrow \infty} \frac{1}{2 T} \int_{-T}^{T}|S(t)|^{2} d t
$$

where $T$ is the period of time and $S$ represents the input signal.

The discrete formats of Equation 2.8 for signal power and noise power are shown in Equation 2.9 and 2.10:

$$
\begin{gathered}
P_{\text {Signal }}=\frac{1}{N} \sum_{n=1}^{N}|S(n)|^{2} \\
P_{\text {Noise }}=\frac{1}{N} \sum_{n=1}^{N}|k(n)|^{2}
\end{gathered}
$$

where $N$ is the total number of samples. $S$ and $k$ represent the input signal (such as in Equation 2.1) and noise signal, respectively.

A criterion to characterize the relative strengths of the signal and the noise is called signal-to-noise ratio, or SNR. There are various types of SNRs, among which power SNR is the one used in this dissertation, and it is the ratio of signal power to noise power. It is a unitless quantity, as shown in Equation 2.11. If SNR equals 1.0, it means the noise power has the same energy as the signal power, which is not desirable because, in such a case, the signal is corrupted by noise with the same power as the signal. The higher the signalto-noise ratio, the better.

$$
S N R=\frac{P_{\text {Signal }}}{P_{\text {Noise }}}
$$

Simulations 5 to 8 illustrate the effect of noise on SRP results. Figure 2.10(a) shows a $4.0 \mathrm{~m}$ by $4.0 \mathrm{~m}$ area with a grid resolution of $0.25 \mathrm{~m}$. The location of the source and accelerometers stays the same in these four simulations, as shown in Figure 2.10(a). Figure 2.10(b) shows the SRP results with no noise. Figures 2.10(c) and 2.10(d) show a different level of noise while the signal power is still dominant. Figure 2.10(e) shows a scenario 
when the noise power exceeds the signal power, but the SRP algorithm still locates the correct vibration source.

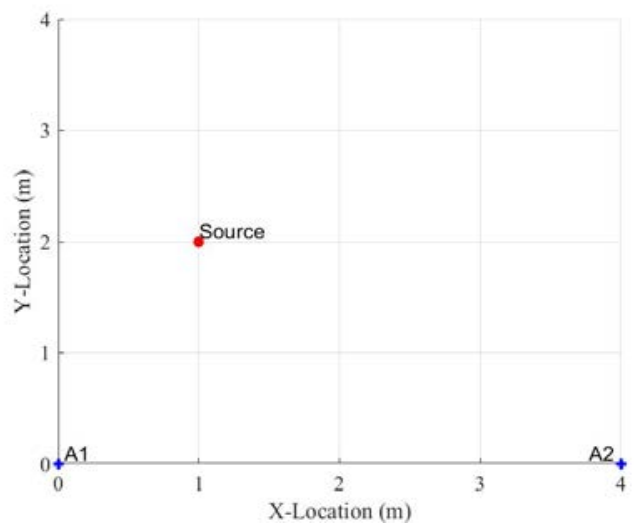

(a) Configuration (Simulation 5, 6, 7, 8)

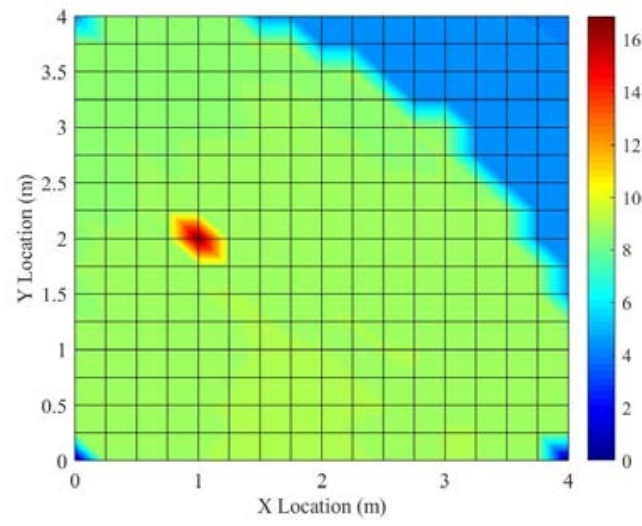

(c) SNR=12.6 (Simulation 6)

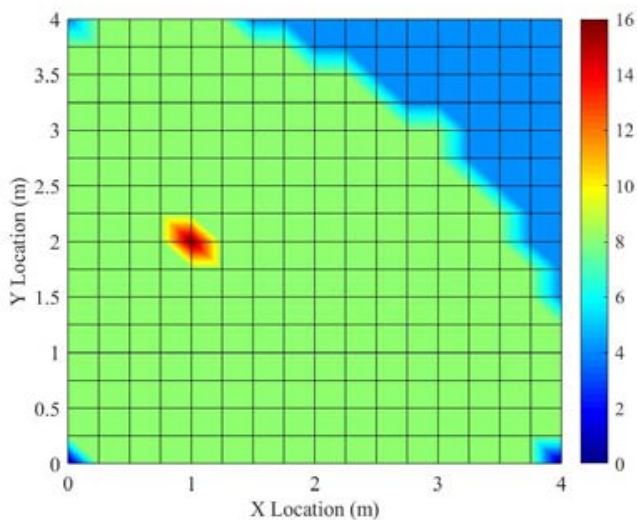

(b) No Additive Noise (Simulation 5)

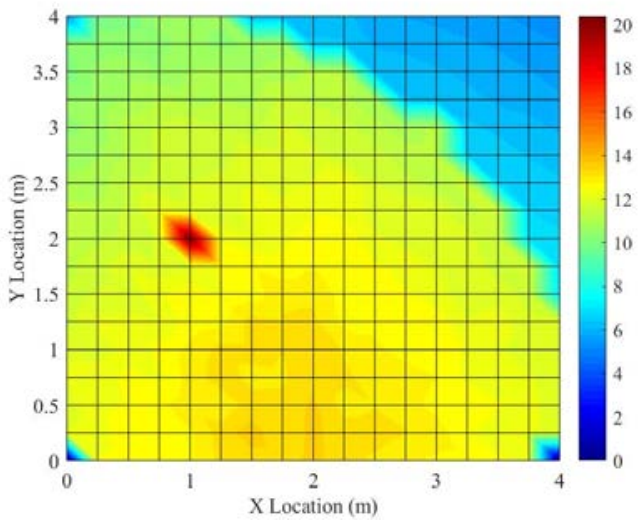

(d) SNR=2.15 (Simulation 7)

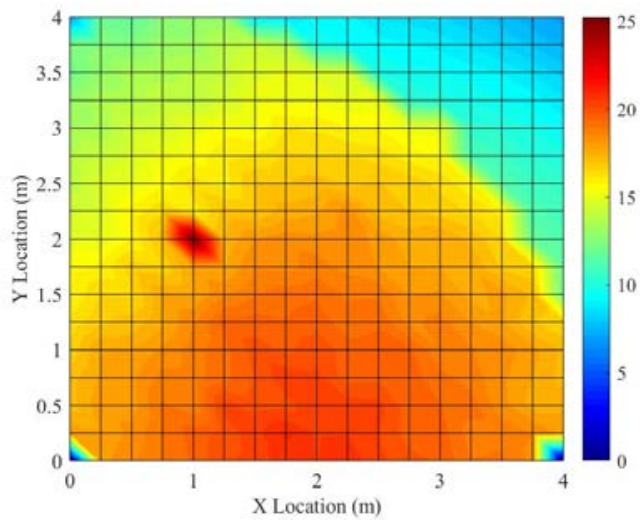

(e) SNR=0.81 (Simulation 8)

Figure 2.10: Effect of Noise on The SRP Results 
The performance of the SRP method to locate the vibration source depends on various parameters, including but not limited to environmental conditions (e.g., structural geometry and boundary conditions of the floor system), spectral contents of the source signal versus dynamic characteristics of the floor (e.g., frequency of excitation signal versus natural frequency of floor), signal processing techniques, and sensor distribution, such as collinear, non-collinear, uniformed, non-uniformed, regular, or irregular configuration (Yu and Donohue 2013).

Benesty et al. (2008) listed some problems that may be addressed by microphone array configuration in localization of sound source as follows: noise reduction, estimation of the number of sources, localization of multiple sources. Without a doubt, the performance of the SRP method is affected by sensor configuration (Feng et al. 2011), including in the application proposed in this dissertation. For example, if the vibration source is located at any point on at the bisector of two accelerometers, as is the case in Simulation 9 (Figure 2.11) and Simulation 10 (Figure 2.12), the SRP plot will not indicate a unique location of the vibration source. In such a case, the resultant SRP plots will show the same steered power for points located on the bisector, as depicted in Figures 2.11 and 2.12. Nevertheless, the SRP plot can still provide clues for engineers to pinpoint where the potential source location might be placed.
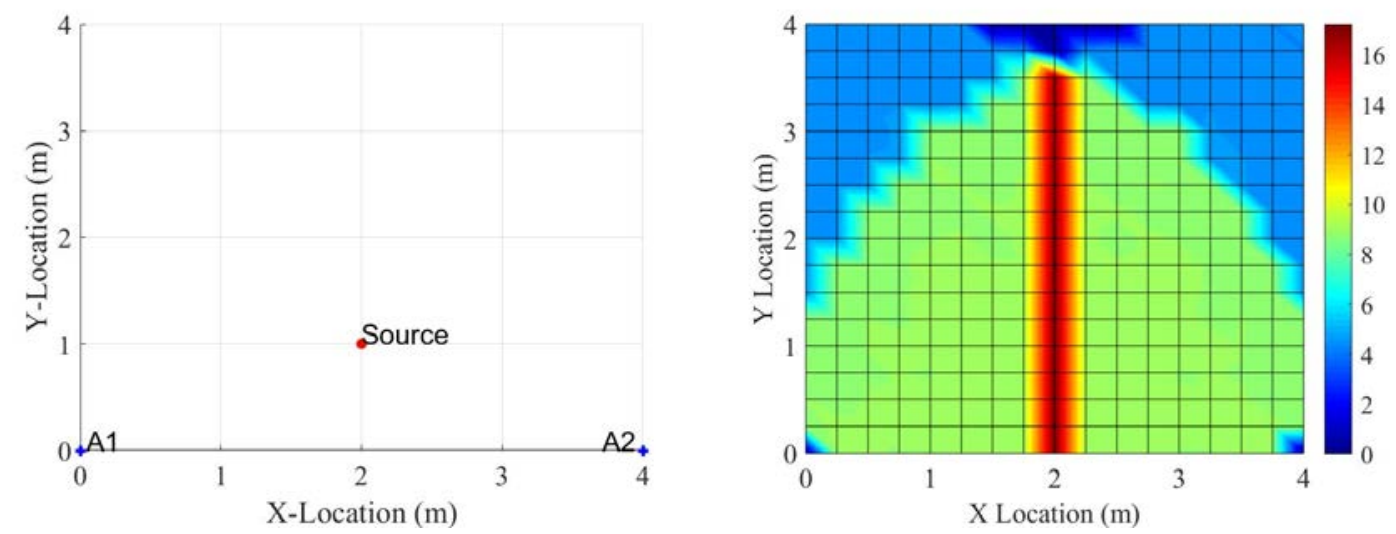

Figure 2.11: Source Located at Bisector of Two Accelerometers and Corresponding SR Plot (Simulation 9) 

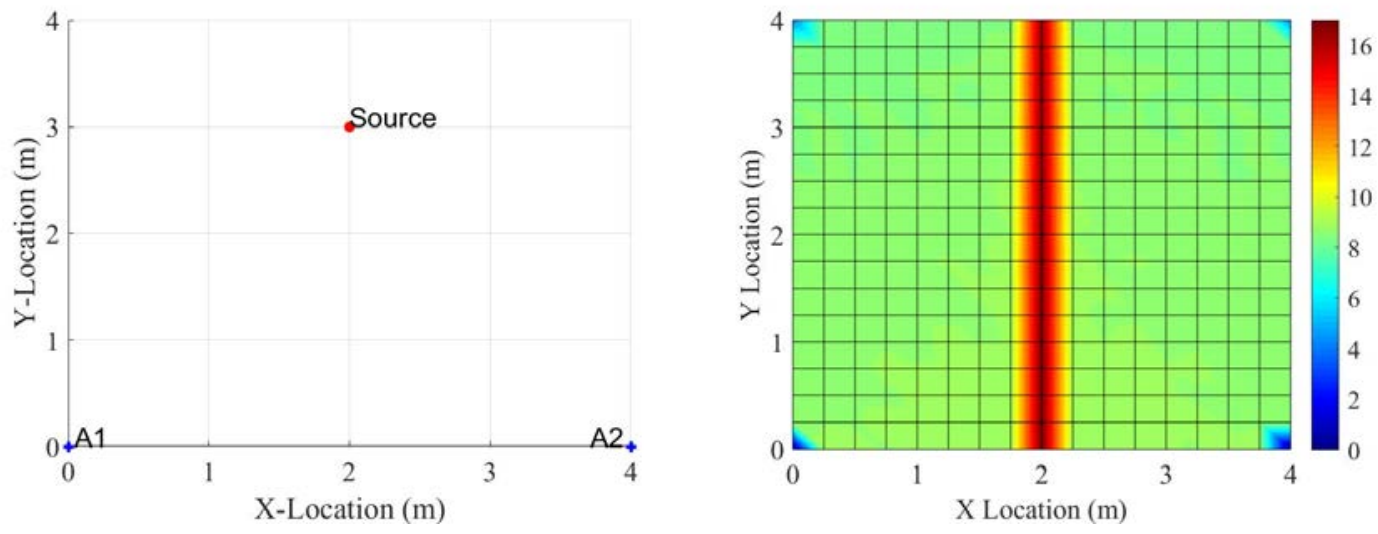

Figure 2.12: Source Located at Bisector of Two Accelerometers and Corresponding SRP Plot (Simulation 10)

The optimum configuration of accelerometers for the purpose presented in this dissertation is one of the future tasks. It was shown by Yu and Donohue (2013) that the use of random sensor configurations outperforms regular and uniform configurations. By changing the location of the A2 accelerometer, resulting in Simulation 11, a clear peak is visible in the SRP plot. Compare Figure 2.13 to Figure 2.11.
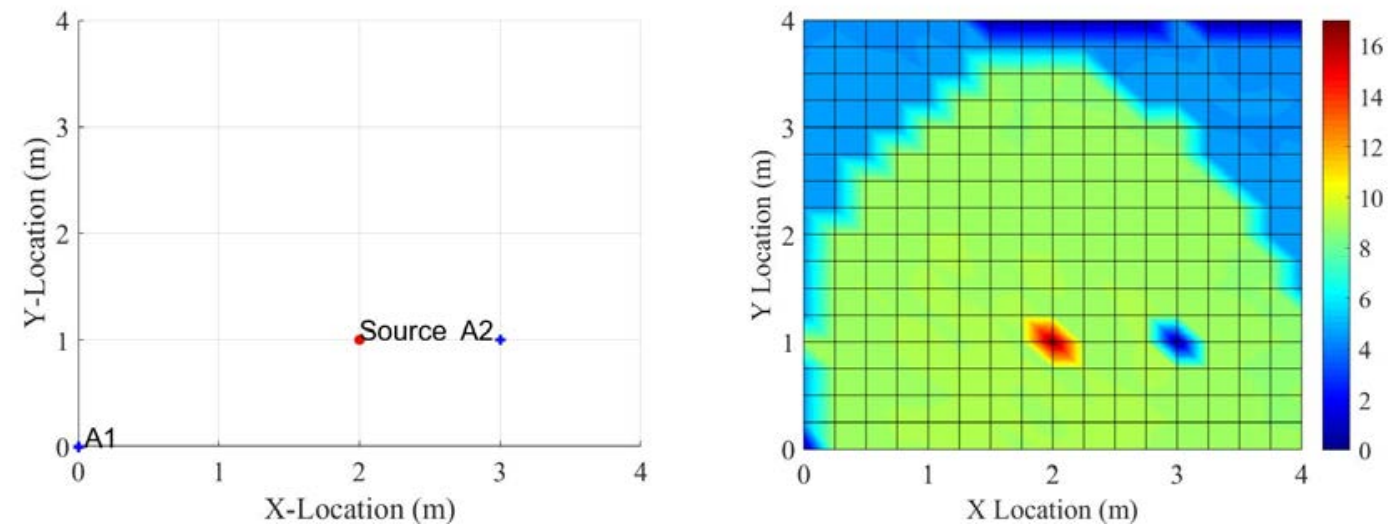

Figure 2.13: Source Located not Placed at Bisector of Two Accelerometers and Corresponding SRP Plot (Simulation 11)

It has just been discussed how judicious sensor placements can potentially improve the performance of the SRP method. For the sake of simplicity, only two accelerometers were used in all simulations mentioned above. The use of more sensors is another way to make the SRP algorithm more robust and enhance localization accuracy. It should be noted that although the high number of vibration sensors is practical, it is not economically viable 
both because vibration sensors are expensive and because the use of a high number of sensors generally requires an analyzer with a high number of input channels, which is also expensive. In addition, the processing of data acquired by a high number of sensors is computationally time-consuming. The objective of this research is to make the applicability of this method as general and practical as possible. In Simulations 12 through 14 , the area of consideration was increased to $8.0 \mathrm{~m}$ by $8.0 \mathrm{~m}$, the sampling frequency rate was set to $1000 \mathrm{~S} / \mathrm{sec}$, the WPS is $1.0 \mathrm{~m} / \mathrm{s}$, and the excitation force is a sinusoid with an amplitude of $2.0 \mathrm{~N}$ and a frequency of $50 \mathrm{~Hz}$. The only difference in Simulations 12 through 14 is the number of accelerometers employed. Figure 2.14 shows a small portion of excitation force, and Figures 2.15 through 2.17 represent different numbers of accelerometers, and their corresponding steered power. The estimated vibration source location is shown with a white arrow, and the localization error, i.e., the distance between the estimated source and the real source location, is added to these SRP plots. In these three simulations, the SRP algorithm accurately found the exact location of the vibration source. However, the quality of SRP plots with the use of a higher number of accelerometers is less ambiguous, and the true peak can be clearly seen.

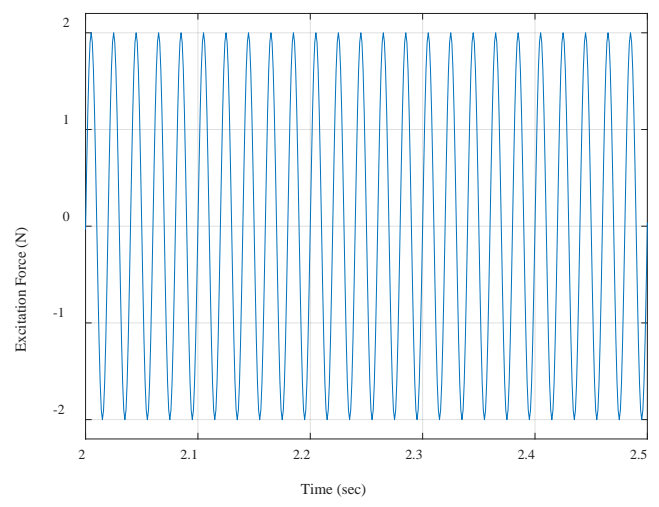

Figure 2.14: Excitation Force for Simulations 12, 13, 14, and 15 

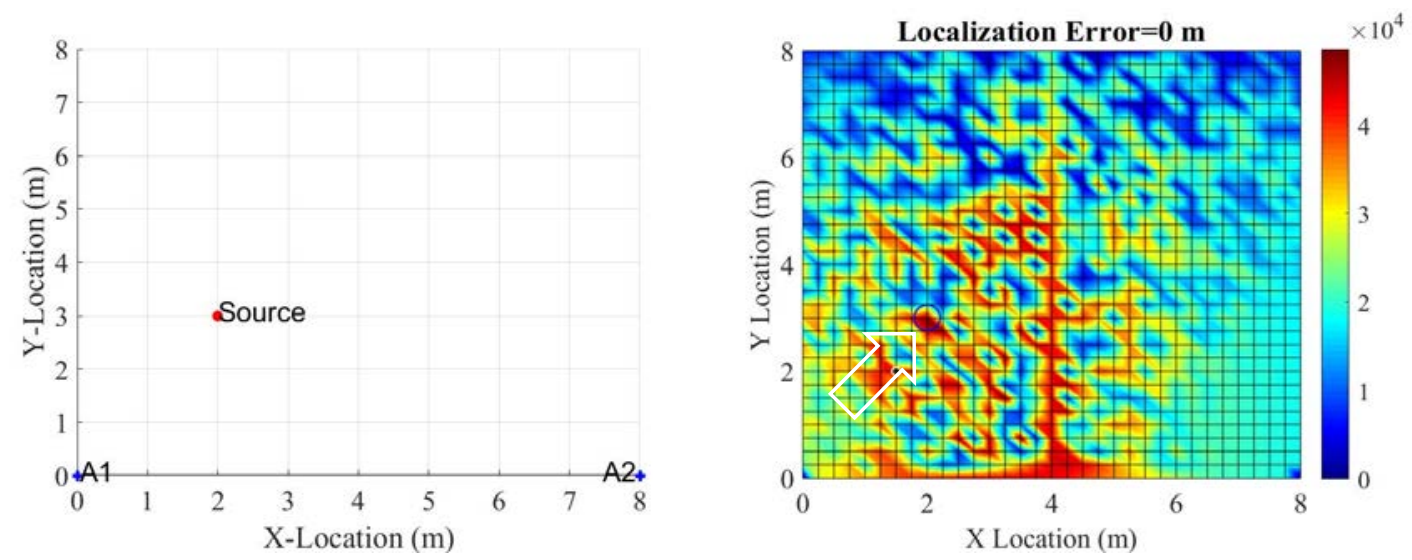

Figure 2.15: Use of Two Accelerometers and SRP Plot for Sinusoid Excitation (Simulation 12)
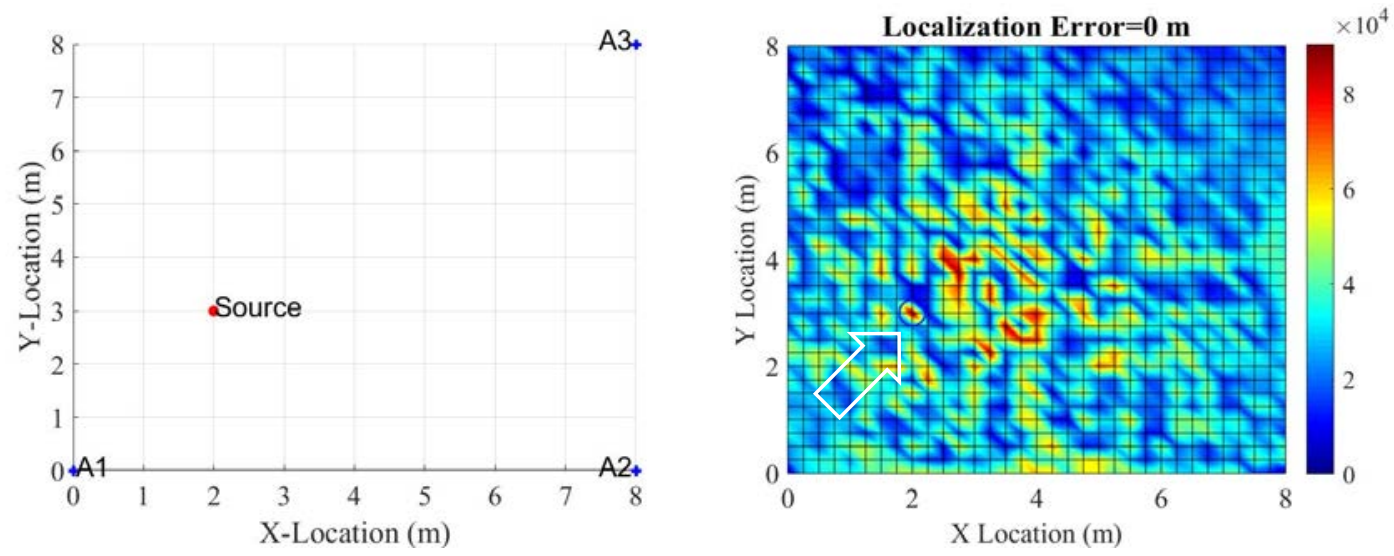

Figure 2.16: Use of Three Accelerometers and SRP Plot for Sinusoid Excitation (Simulation 13)
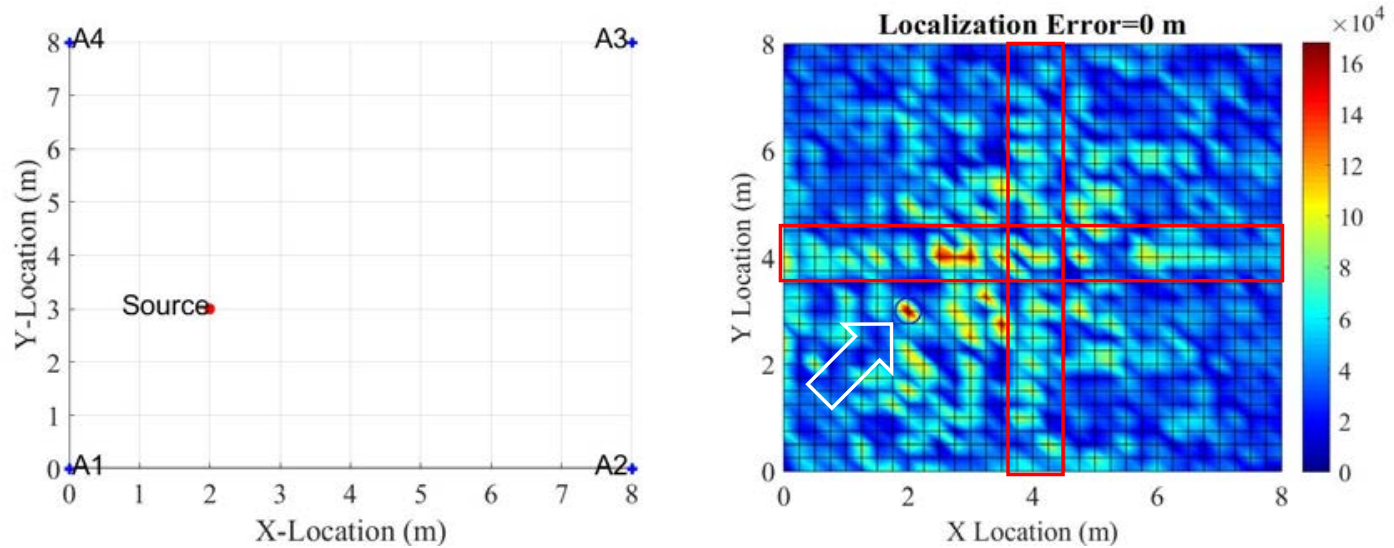

Figure 2.17: Use of Four Accelerometers and SRP Plot for Sinusoid Excitation (Simulation 14) 
As mentioned previously, the vibration source was correctly identified in Simulation 14. However, there are some other peaks, especially at the bisector of the accelerometers, as shown by the red rectangles in Figure 2.17. In Simulation 15, Figure 2.18, the SRP plot is for an irregular distribution of sensors. It illustrates that if bisectors do not overlap with each other, the quality of the SRP plot is a little better compared to Simulation 14.
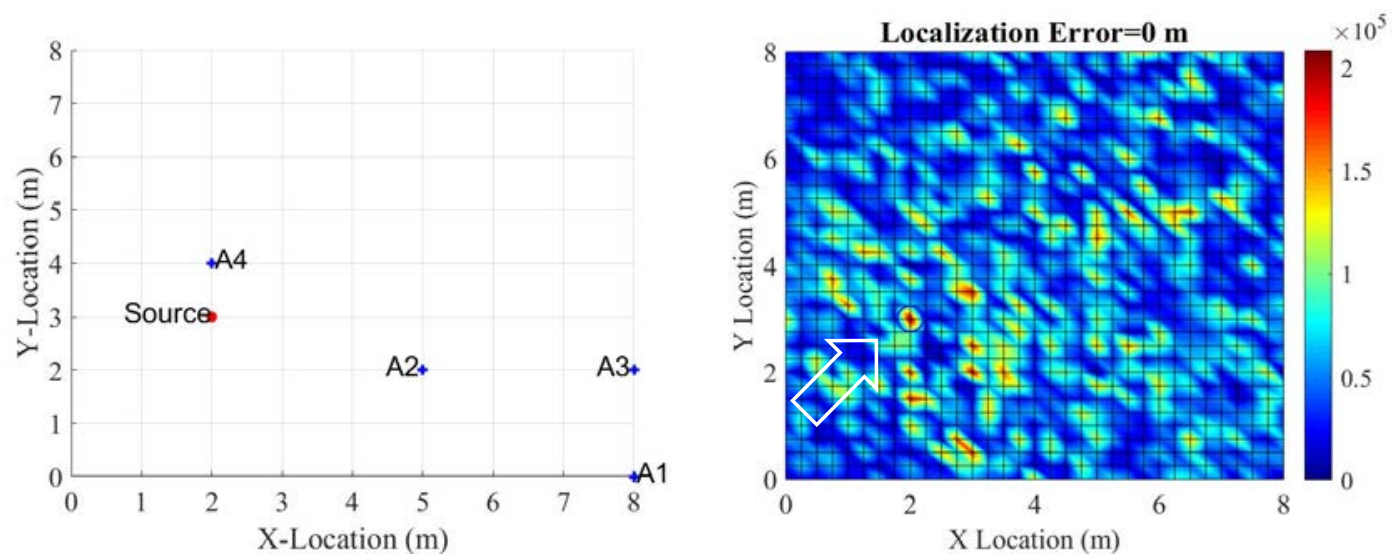

Figure 2.18: Effect of Irregular Distribution of Accelerometers in SRP Plots for Sinusoid Excitation (Simulation 15)

So far, for all simulations mentioned above, the excitation force was either a single pulse or a sinusoid. This algorithm also works for random excitation forces. In Simulation 16, Figure 2.19(a) shows a portion of a random excitation force applied at location $(2,3)$ meter on Figure 2.19(b); Figure 2.19(b) also shows the location of the accelerometers; and Figures 2.19(c) and 2.19(d) represent the 3D view and plan view of the corresponding steered power. This demonstrates how the quality of the SRP plot is affected by different excitation forces. 


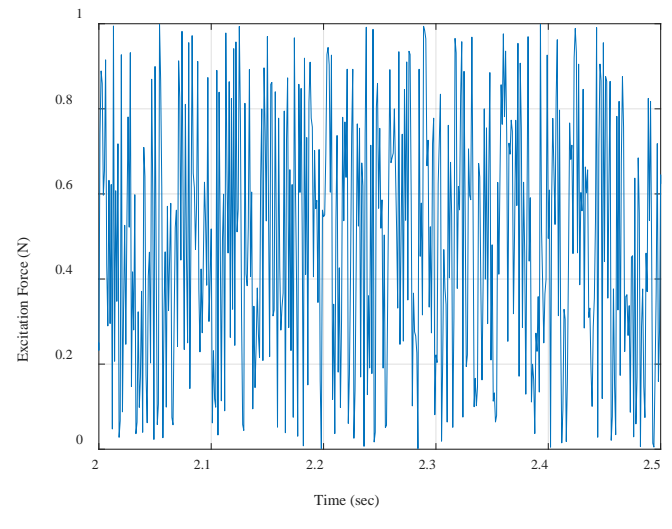

(a) Random Excitation

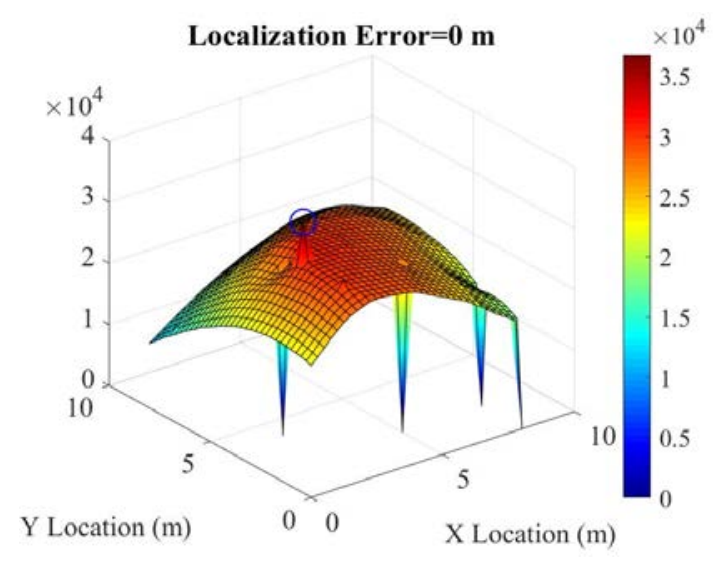

(c) SRP Result (3D View)

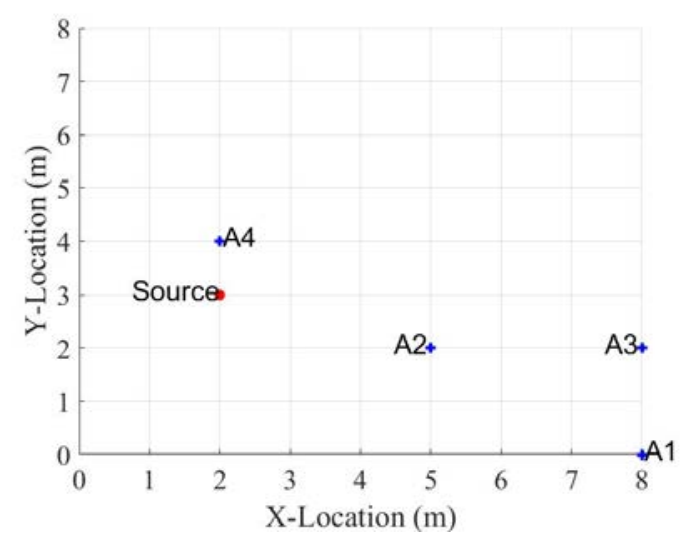

(b) Configuration

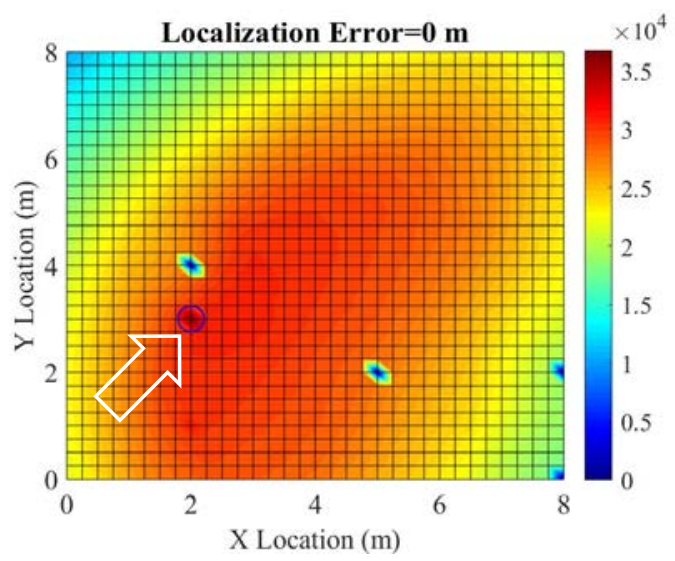

(b) SRP Result (Plan View)

Figure 2.19: Performance of SRP Algorithm with Random Noise (Simulation 16)

In Simulations 1-16 described above, the effect of attenuation was not considered, and the SRP algorithm was based on equal contributions of all accelerometers in the absence of attenuation. However, with the presence of attenuation, it makes sense to assume that accelerometers closer to the source receive more energy than those farther away. Therefore, a mathematical model is needed to give more weights to closer accelerometers than farther ones regarding their distance to the selected candidate location. For this purpose, inverse distance weighting is used, as shown in Equation 2.12:

$$
w_{i}=\frac{\frac{1}{d_{A_{i}}^{b}}}{\sum_{i=1}^{N} \frac{1}{d_{A_{i}}^{b}}}
$$


where $w_{i}$ is the weight for $i^{\text {th }}$ accelerometer, $d_{A i}$ is the distance between the selected candidate location and $i^{\text {th }}$ accelerometer using Equation 2.4., and $b$ is the decaying power, which can be any positive value. The greater the value of $b$, the more weight will be given to the closer sensors, and less weight will be given to the farther ones.

To better understand Equation 2.12, see the example configuration shown in Figure 2.20 with four uniformly distributed accelerometers with a collinear configuration. A1 is the closest accelerometer to the source in this example. Table 2.2 provides different weights for the various accelerometers as a function of $b$, as shown in Figure 2.20. If $b$ equals zero, this means an equal weighting distribution for all accelerometers. If $b$ equals $1.0, w_{1}, w_{2}, w_{3}$, and $w_{4}$ for A1, A2, A3, and A4 accelerometers will be $48 \%, 24 \%, 16 \%$, and $12 \%$, respectively, using Equation 2.12 .

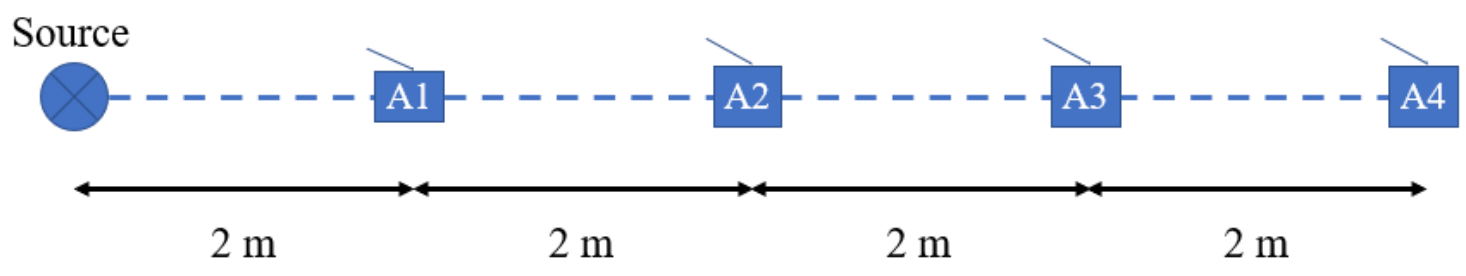

Figure 2.20: Visual Example to Show Inverse Distance Weight

Table 2.2: Effect of $b$ on Inverse Distance Weighting

\begin{tabular}{|c|c|c|c|c|c|c|c|c|}
\hline \multirow{2}{*}{$w_{i}$} & \multirow{2}{*}{$\begin{array}{c}d_{i} \\
(\mathbf{m})\end{array}$} & \multicolumn{7}{|c|}{$\boldsymbol{v}$} \\
\hline & & 0 & 0.25 & 0.5 & 0.75 & 1 & 2 & 4 \\
\hline$w_{1}$ & 2 & $25 \%$ & $30 \%$ & $36 \%$ & $42 \%$ & $48 \%$ & $70 \%$ & $93 \%$ \\
\hline$w_{2}$ & 4 & $25 \%$ & $25 \%$ & $25 \%$ & $25 \%$ & $24 \%$ & $18 \%$ & $6 \%$ \\
\hline$w_{3}$ & 6 & $25 \%$ & $23 \%$ & $21 \%$ & $18 \%$ & $16 \%$ & $8 \%$ & $1 \%$ \\
\hline$w_{4}$ & 8 & $25 \%$ & $21 \%$ & $18 \%$ & $15 \%$ & $12 \%$ & $4 \%$ & $0 \%$ \\
\hline & Sum & $100 \%$ & $100 \%$ & $100 \%$ & $100 \%$ & $100 \%$ & $100 \%$ & $100 \%$ \\
\hline
\end{tabular}

When $b$ has a value greater than one, Equation 2.12 drastically decreases the contribution of the farther sensors. Therefore, for the application presented in this study, $b$ is kept between zero and one. The effect of inverse distance weighting will be shown in Chapter 3 via a real experiment. 


\subsection{Estimation of Major Parameter, Wave Propagation Speed}

As discussed in Section 2.2, for computation of the steered power for all candidate locations, all parameters are known in Equations 2.1 to 2.7 except the WPS. The accuracy of the SRP method relies on the correct estimation of WPS, which is the focus of this section. Estimation of WPS using vibration measurements is a major challenge due to structural variation, dispersion, attenuation, and multipath effect. Although the exact computation of WPS is possible, it would require a complete understanding of the complex interaction between propagated waves and the concrete floor system (Royvaran et al. 2020). Indeed, there is no simple model for estimation of WPS based on the physical properties of the concrete floor, e.g., damping, Young modulus, stiffness, and density (Chen et al. 2002). The variation of estimated WPS, type of applied dynamic load, and the objective of the study in some studies are shown in Table 2.3.

Table 2.3: WPS Estimation is Selected Studies

\begin{tabular}{|c|c|c|c|}
\hline Studied by & Objective of the Study & $\begin{array}{c}\text { Type of Dynamic } \\
\text { Load }\end{array}$ & $\begin{array}{c}\text { WPS } \\
\text { (m/s) }\end{array}$ \\
\hline Richman et al. 2001 & Footstep localization & Person jumped & 168 \\
\hline $\begin{array}{c}\text { Schloemann et al. } \\
2015\end{array}$ & Hammer strike localization & Hammer strike & 914 \\
\hline Lee et al. 2009 & $\begin{array}{c}\text { Estimation of indoor physical } \\
\text { activity }\end{array}$ & - & $>1500$ \\
\hline Bahroun et al. 2014 & Footstep localization & - & $>1000$ \\
\hline
\end{tabular}

Nevertheless, some level of inaccuracy is acceptable in the estimation of WPS. Before giving in-depth explanations of WPS estimation and seeing the effect of an inaccurate WPS estimate of the SRP plot, another set of simulations will be shown here. For all simulations mentioned in Section 2.2, the WPS was $1.0 \mathrm{~m} / \mathrm{s}$. In Simulation 17, the area under consideration has been increased to $10.0 \mathrm{~m}$ by $10.0 \mathrm{~m}$. The true WPS is $10 \mathrm{~m} / \mathrm{s}$. Two types of errors are presented in Figure 2.21: WPS estimate error and corresponding localization error in the SRP plot. Figure 2.21(a) shows the configuration of accelerometers and the source. Figure 2.21(c) shows the SRP plot with a correct WPS estimate of $10 \mathrm{~m} / \mathrm{s}$. Figures 2.21(b) and 2.21(d) show an inaccurate estimation of WPS, and the localization errors in these two cases are $3.0 \mathrm{~m}$ and $1.0 \mathrm{~m}$, respectively. The estimated source localization by the SRP algorithm is shown by a white circle in these 
figures. Figure 2.21(e) shows localization errors with respect to different WPS values in the form of a bar plot.

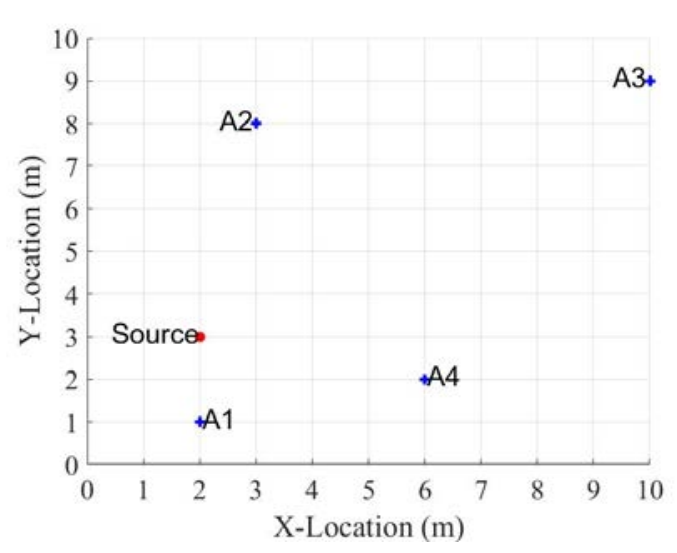

(a) Configuration

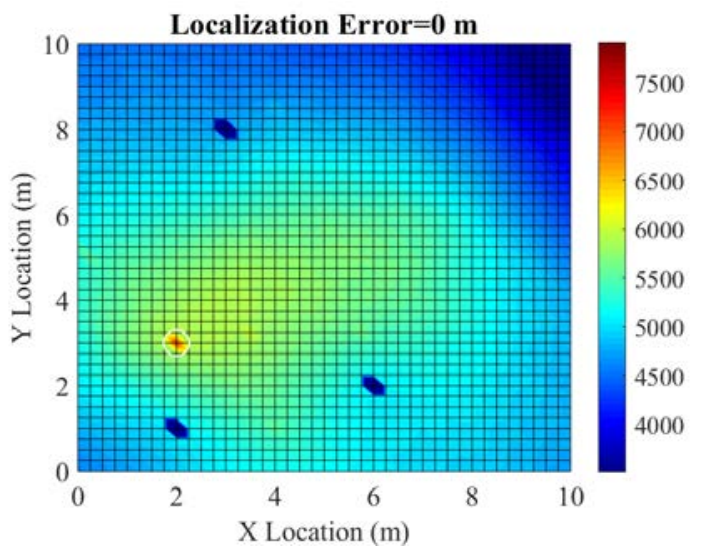

(c) Estimated WPS=10 m/s (Correct Estimation)

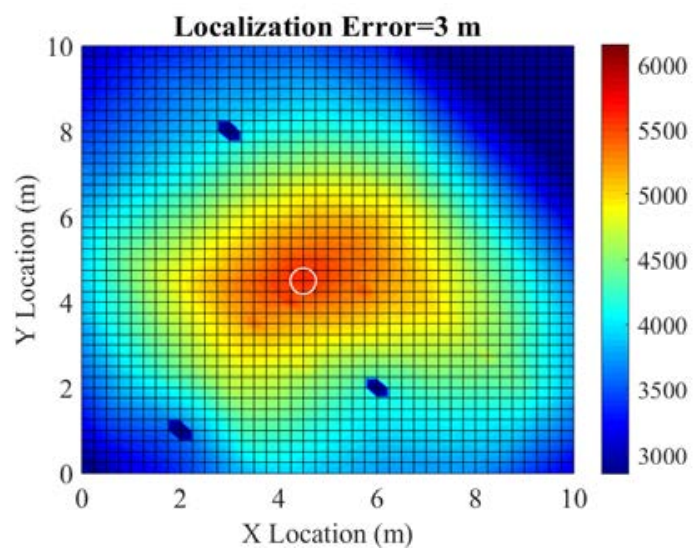

(b) Estimate WPS $=7 \mathrm{~m} / \mathrm{s}$

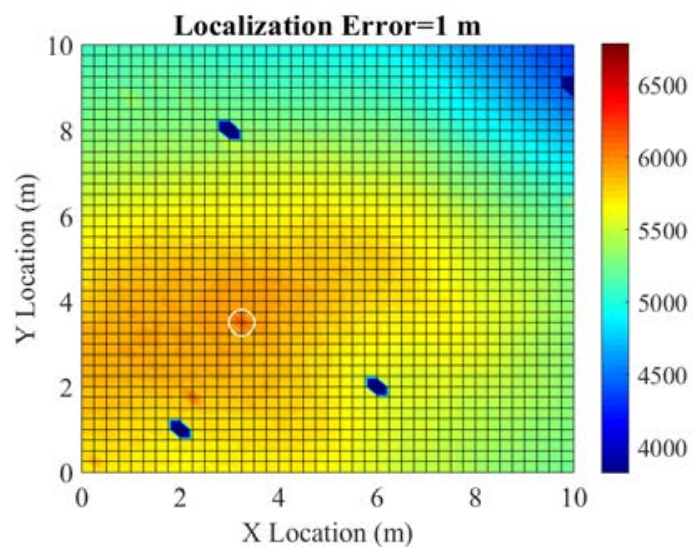

(d) Estimated WPS=14 m/s

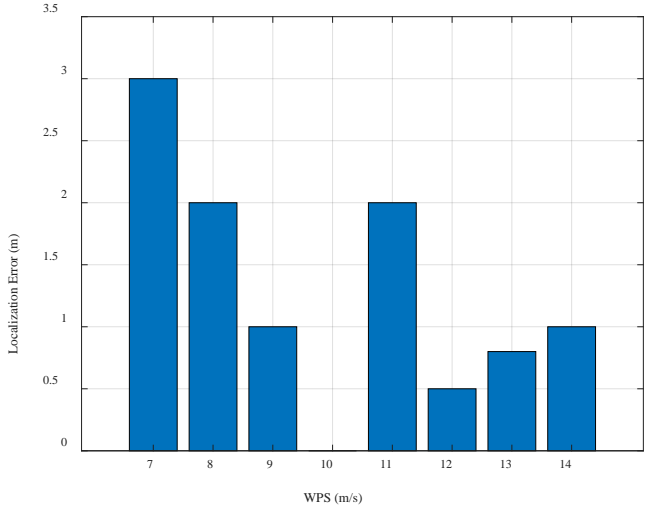

(e) Localization Error Versus WPS

Figure 2.21: Effect of Inaccurate WPS Estimation on the Accuracy of SRP Algorithm (Simulation 17) 
Estimation of WPS is a prerequisite step for the utilization of the SRP method for each floor. Therefore, a separate set of experiments needs to be conducted, and the collected data will be post-processed for this purpose. In these experiments, the location of the vibration source, location of accelerometers, and time-domain excitation force data are all given. Equation 2.13 is based on the kinematic equation to predict the motion of an object and can be utilized to estimate WPS:

$$
W P S=\frac{D_{i}}{T D}
$$

where $D_{i}$ is the distance between the excitation source and the location of $A_{i}$ accelerometer, and $T D$ denotes time delay. $D_{i}$ is known in this equation. After estimating the time delay, the WPS can be estimated using Equation 2.13.

Various time delay estimation methods are available in the literature (Omologo and Svaizer, 1994). In the research described herein, two have been adapted: the time-domain approach and the frequency-domain approach. The time-domain approach aims to measure the relative time difference of arrival, or time delay, between a set of several pairs of sensors using direct cross-correlation in the time-domain (Benesty et al. 2004). In the frequency-domain approach, cross-spectrum phase analysis is used to estimate the relative time delay. This method has already been applied in acoustic event localization, and it has been shown to work well in noisy and reverberant acoustical environments (Omologo and Svaizer, 1997).

This section is organized as follows. Section 2.3.1 reviews the previous studies for computation of time delay. Sections 2.3.2 and 2.3.3 provide the details of two approaches utilized in this chapter to estimate time delay and then WPS.

\subsubsection{Previous Studies and Challenges in Time Delay Estimation}

As mentioned in Chapter 1, one of these challenges is that the signal can be mixed with other vibration sources (Shi and Ming 2016), such as human walking. Another challenge is the multipath propagation effect (Section 1.2.4.1), where receivers acquire attenuated and delayed replicas of the source signal reflected from the boundaries (Benesty et al. 2004). This effect may be observable in applications, such as concrete floors, where the sensor receivers get the excitation signal via two or more paths in the presence of 
obstacles such as CMU walls, openings, columns, beams, or girders (Mirshekari et al. 2021). Additionally, wave propagation in dispersive and attenuative media has been discussed in Section 1.2.4. In summary, then, dispersion is a frequency-dependent phenomenon in which frequency components travel at different speeds (Sulaiman et al. 2010). Philippidis and Aggelis (2005) have shown that due to the inhomogeneous nature of concrete, i.e., that it consists of cement, sand, fine and coarse aggregates, water, and air bubbles, attenuation varies for different frequencies traveling a constant distance through a concrete slab. Moreover, in many localization methods, propagation speed is assumed to be constant in all directions. However, in Chapter 3 of this study, it will be shown that this assumption is not valid for the tested concrete floor.

All the challenges mentioned above can distort the shape of received signals transmitted by the source signal. However, the degree of this distortion has not been studied in-depth, specifically for composite floors.

\subsubsection{Time Delay Estimation Using Time-Domain Approach}

This section provides a description of time delay estimation using time-domain analysis. The time delay for each accelerometer is the time required for the vibration waves to travel from the source of vibration to the accelerometer through the concrete floor. Equation 2.14 shows the cross-correlation equation between excitation signal, $A_{E}$, and the signal received by the $i^{\text {th }}$ accelerometer, $A_{i}$. The method of cross-correlation, denoted below by $R$, is the most popular technique for estimating time delay (Knapp and Carter, 1976) by measuring how the excitation signal, $A_{E}$, and the received signal, $A_{i}$, compare when offset by various values of the lag, $l$. The lag refers to how far the two signals are offset in time. The significant advantage of the cross-correlation function in the timedomain is demonstrated when the excitation signal, $A_{E}$, is unknown or changing (Donohue

et al., 2007). Obviously, the maximum correlator, maximum value of $R$, corresponds to the lag, $l_{R \max }$, when the received signal has the best match with the excitation signal, resulting in the time delay estimate.

$$
R(l)=\sum_{n=0}^{N-l-1} A_{E}(n) A_{i}(n+l)
$$


where $N$ is the number of samples for each signal, and $l$ is the lag between the excitation signal and the $A_{i}$ accelerometer.

The computation of time delay, $T D$, is expressed in Equation 2.15 where the lag corresponds to the maximum cross-correlation, as shown by $l_{R m a x}$ :

$$
T D_{t}=\frac{l_{R \max }}{F_{s}}
$$

Here, the $l_{R \max }$ is the lag in the unit of samples, and $F_{s}$ is the sampling frequency in the unit of samples per second. Therefore, the unit for time delay, $T D_{t}$, is seconds. The subscript $t$ represents the time delay estimate using the time-domain approach.

\subsubsection{Time Delay Estimation Using the Frequency-Domain Approach}

This section provides a description of time delay estimation by an analysis in the frequency-domain. This approach has been proven to work well in the localization of acoustic events using microphone arrays in noisy and reverberant environments (Omologo and Svaizer, 1997). This dissertation is applying it for the first time to process acceleration data acquired from a concrete floor to the writer's knowledge. In this approach, all signals are transferred from the time-domain to the frequency-domain. The excitation signal is designated as the reference signal. Cross-spectrum, $\hat{P}(f)$, between the excitation signal, $\hat{A}_{E}$, and the $i^{\text {th }}$ accelerometer, $\hat{A}_{i}$, and is expressed in Equation 2.16. In a basic sense, the Fourier transformation of cross-correlation provides cross-spectrum. Hereafter, the ${ }^{\wedge}$ sign demonstrates the signal in the frequency-domain:

$$
\hat{P}(f)=\hat{A}_{i}^{*}(f) \hat{A}_{E}(f)
$$

where $\hat{A}_{i}$ and $\hat{A}_{E}$ are the Fourier transform of $A_{i}$ and $A_{E}$, and superscript * denotes the complex conjugate.

The time delay is proportional to the slope of the resulting phase of the crossspectrum in the frequency range of interest. The gradients of the phase spectrum, $G$, are expressed in Equation 2.17:

$$
G(f)=\frac{\theta(f+1)-\theta(f-1)}{2 \Delta f}
$$


where $\theta(f)$ is the unwrapped phase spectrum, and $\Delta f$ is the frequency resolution. The time delay estimation, $T D_{f}$, is expressed in Equation 2.18. This equation is weighted inversely with the cross-spectrum magnitude, which has been proven to be effective for the multipath propagation problem:

$$
T D_{f}=\frac{\sum_{f=f_{l}}^{f_{u}}|\hat{P}(f)| G(f)}{2 \pi \sum_{f=f_{l}}^{f_{u}}|\hat{P}(f)|}
$$

where $f_{l}$ and $f_{u}$ are summation limits corresponding to the lower and upper spectral limits to emphasize the frequency range of interest. The subscript $f$ represents the time delay estimate using the frequency-domain approach. More details about Equation 2.16 to 2.18 are provided by Omologo and Svaizer (1994), Omologo and Svaizer (1997), Donohue et al. (2007), and Sulaiman (2010). 


\section{CHAPTER 3: EXPERIMENTAL PROGRAM}

\subsection{Introduction}

The concept of the SRP method and related equations were provided in Chapter 2. In this chapter, the performance of the SRP method and the use of these equations on a full-scale building will be described.

Section 3.2 illustrates some details about the tested floor. Section 3.3 will provide some details about the list of equipment and their applications in this research. The Frequency Response Function (FRF) of the floor is discussed in Section 3.4.

As already mentioned in Chapter 2, the wave propagation speed (WPS) is the prerequisite parameter in the SRP method. Therefore, two different approaches are proposed in Section 3.5 to estimate the WPS. Then, the application of the SRP method on a full-scale building will be provided in Section 3.6.

\subsection{Specimen Description}

This section describes the required information about the experimental work done in this dissertation. Figure 3.1 shows the structural drawing of the second floor in the Oliver H. Raymond (OHR) building on the University of Kentucky campus. The concrete thickness is $7.6 \mathrm{~cm}$. The joist stem depth and width are approximately $40.6 \mathrm{~cm}$ and 15.2 $\mathrm{cm}$, respectively. The spacing between stems is $38.1 \mathrm{~cm}$. The concrete strength is 4000 psi. Figure 3.2 illustrates two sectional views of column lines $\mathrm{E}$ and F. The test was conducted in a single bay between column lines 3-4 and F-E. The specimen was $8.4 \mathrm{~m}$ by $13.4 \mathrm{~m}$ in area. This area is used as a computer lab, and the furniture used mostly includes desks, each of which has a computer and six chairs. 


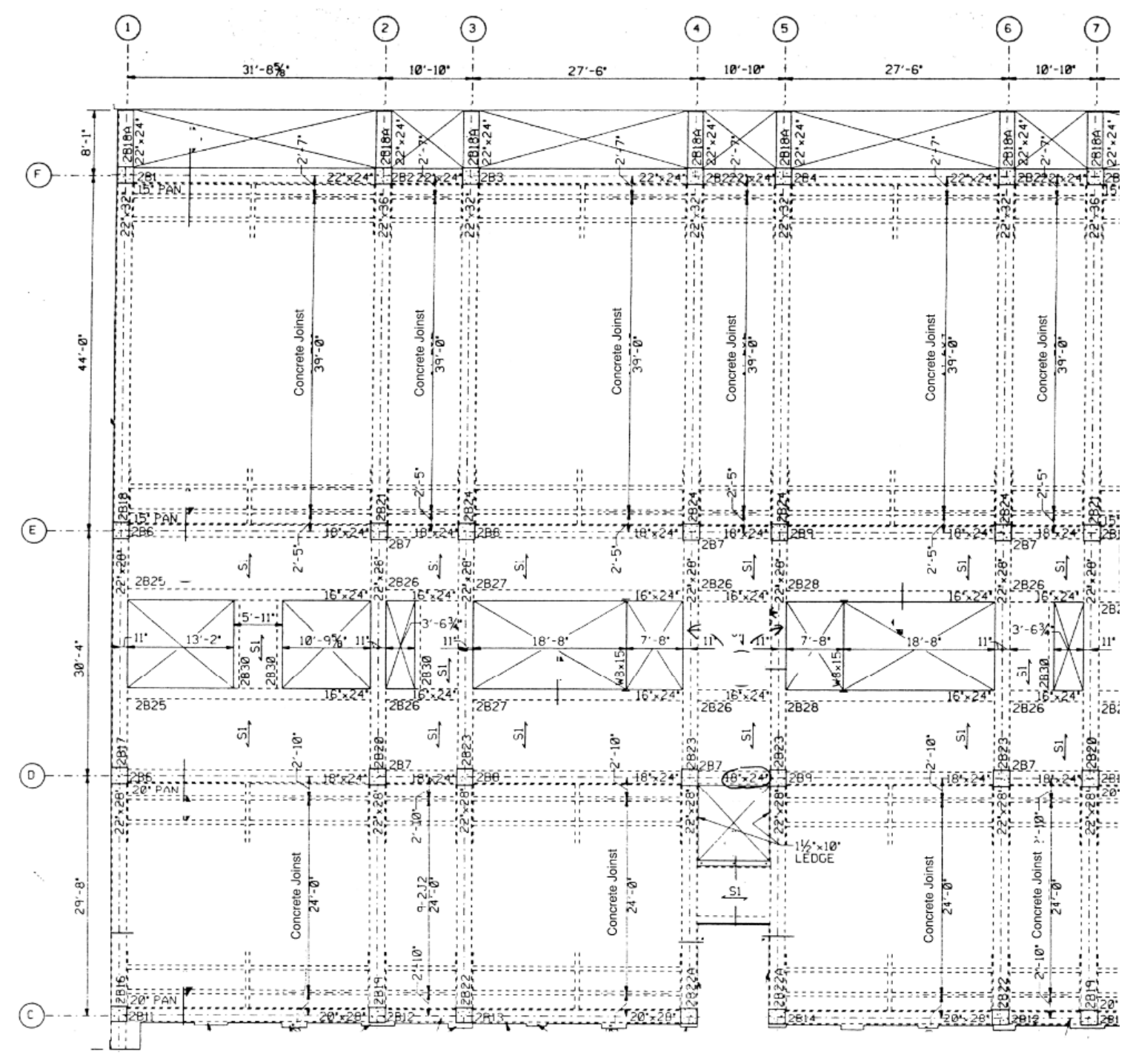

Figure 3.1: Structural Drawing of the Second Floor, OHR Building, University of Kentucky

(F)

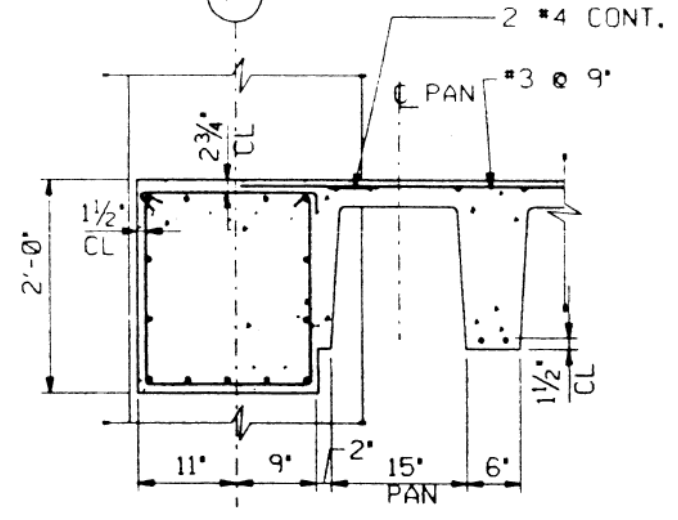

(E)

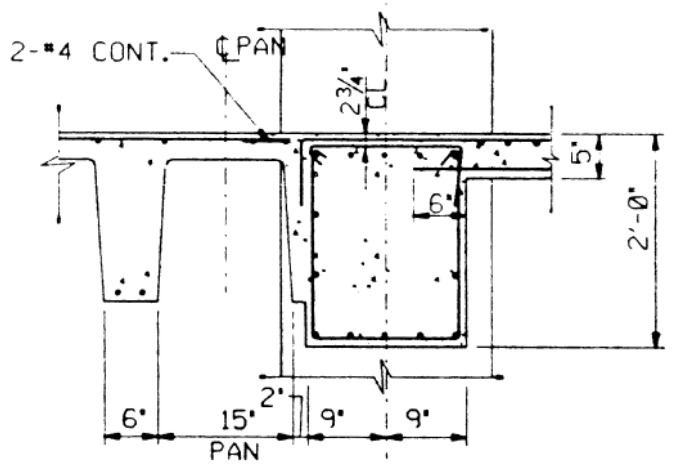

Figure 3.2: Section View in the North-South Direction Along with Column-Line E and F 


\subsection{Dynamic Testing Equipment}

In Sections 3.2.1 to 3.2.3 below, a short technical summary of the equipment used in this dissertation will be given.

\subsubsection{Electrodynamic Shaker}

The vibration exciter used in this research is an APS ELECTRO-SEIS Model 400 shaker manufactured by APS Dynamics (see Figure 3.3). This electrodynamic shaker is particularly suitable for the excitation of large structures, such as concrete slabs or even bridges, to study their dynamic response characteristics. Some capabilities of this shaker are listed in Table 3.1.

Table 3.1: Some Capabilities of APS ELECTRO-SEIS Model 400

\begin{tabular}{|c|c|}
\hline Vibration Type & Sinusoid, Swept, random, or impulsive force waveforms \\
\hline Vibration Direction & Either Vertical or Horizontal \\
\hline Max. Force & $445 \mathrm{~N}(100 \mathrm{lbf})$ \\
\hline Max. Velocity & $1,000 \mathrm{~mm} / \mathrm{s}(39 \mathrm{in} / \mathrm{s})$ \\
\hline Max. Frequency Range & $200 \mathrm{~Hz}$ \\
\hline Entire Assembly Weight & $107 \mathrm{~kg}(236 \mathrm{lb})$ \\
\hline
\end{tabular}

The shaker has the following three limitations:

- Due to its high weight and sensitivity, moving the shaker is difficult and costly.

- The shaker might cause uncomfortable vibrations for occupants. Therefore, the vibration test should be done after hours, overnight, or on weekends. This avoids any extraneous vibration source, e.g., the use of elevator, door slamming, and walking.

- $\quad$ Setup and disassembly are required, which are relatively time-consuming.

This shaker was used in this dissertation for the following applications:

- Determination of dynamic characteristics of the structure, e.g., natural frequencies

- Assessment of the floor vibration level due to applied excitation force by the shaker

- Vibration simulation on the tested floor

- Study of vibration wave propagation estimation 


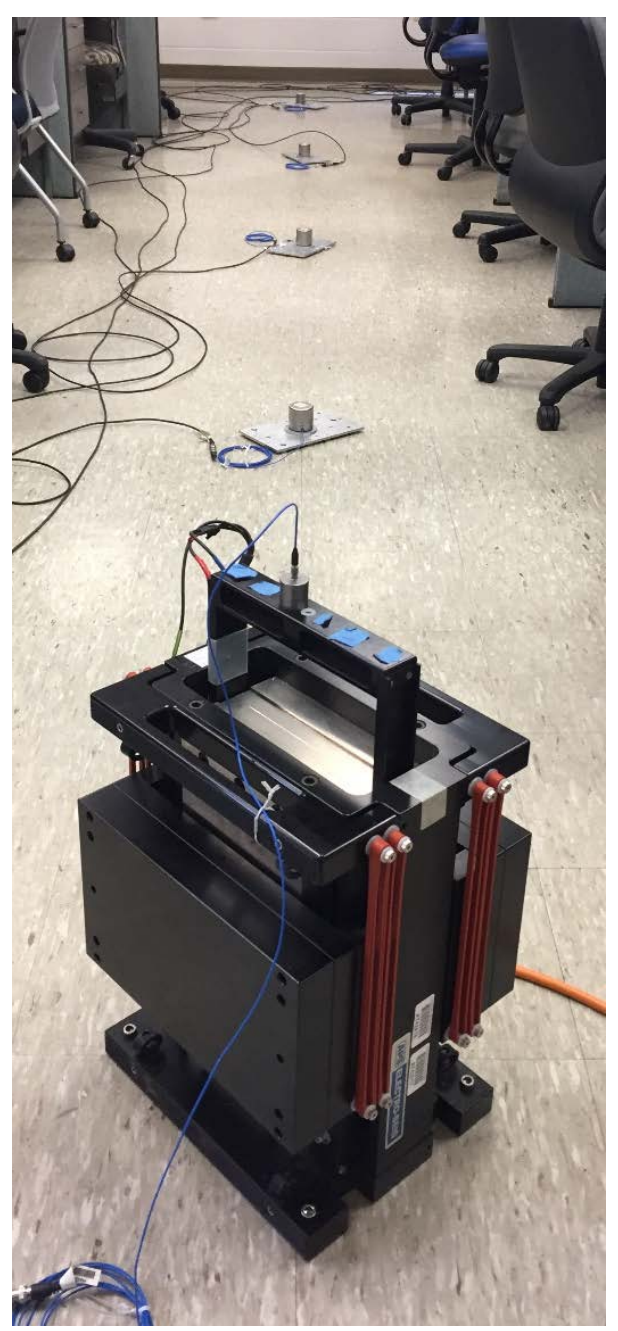

Figure 3.3: APS ELECTRO-SEIS Model 400 shaker

The shaker vibration level is configured by an NV-Gate software program, a multichannel spectrum analyzer, and an APS 145 power amplifier, as illustrated in Figure 3.4. 


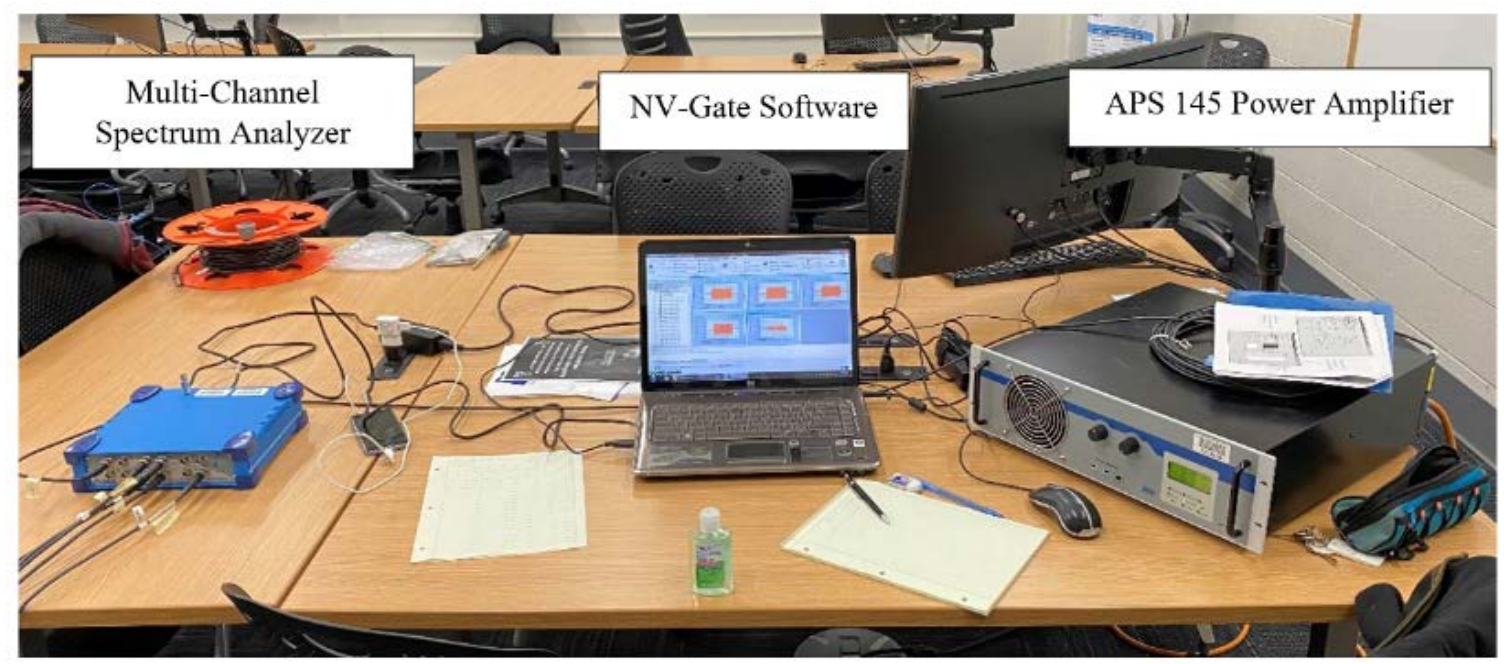

Figure 3.4: Multi-Channel Spectrum Analyzer, NV-Gate Software program, and APS 145 Power Amplifier

\subsubsection{Multi-Channel Spectrum Analyzer}

The 8-Channel OR35 spectrum analyzer and the NV-Gate software program (both produced by OROS manufacture) were used in this research to generate the output excitation signal for the shaker and acquire time-domain acceleration data from accelerometers. The NV-Gate software program not only can record the time-domain waveform but also has the ability to compute the Fast Fourier Transform, auto-spectrum, cross-spectrum, frequency response function, and coherence function.

The main role of the multi-channel analyzer and NV-Gate software program is to convert analog acceleration data into digital form in order to make it readable for the computer. Accordingly, this process consists of digitization and quantization. The digitization process, also known as sampling, discretizes data in the time-domain - i.e., $\mathrm{x}$-axis data - and defines the time resolution. Since the continuous analog data is converted into a finite number of data points, some data between time intervals will be lost. At the same time, the quantization procedure discretizes the amplitude values, i.e., $\mathrm{y}$ axis data. Indeed, the continuous analog amplitude values corresponding to each data point are rounded off after digitization. The negative effects of digitization due to data lost between two consecutive data points and quantization due to rounding off are very negligible with the use of advanced equipment in the present study and, therefore, were 
ignored. In sum, in this study, the sampling frequency rate was set to 10,240 samples per second — less than 0.0001 second time resolution — with the digital-to-analog converter providing 24-bits, i.e., $2^{24}$, of quantization resolution.

\subsubsection{Accelerometers}

The acceleration data was measured using 393C seismic accelerometers manufactured by PCB Piezotronics. These accelerometers have a frequency range of 0.025 to $800 \mathrm{~Hz}$ with a less than $5 \%$ deviation. Their sensitivity is $1000 \mathrm{mV} / \mathrm{g}$, and their maximum measurement range is $2.5 \mathrm{~g}$. The accelerometer weights less than $1.0 \mathrm{~kg}$ and has a wide base. Also, the carpet was extremely firm. Therefore, in this study, the accelerometers were placed on the carpet.

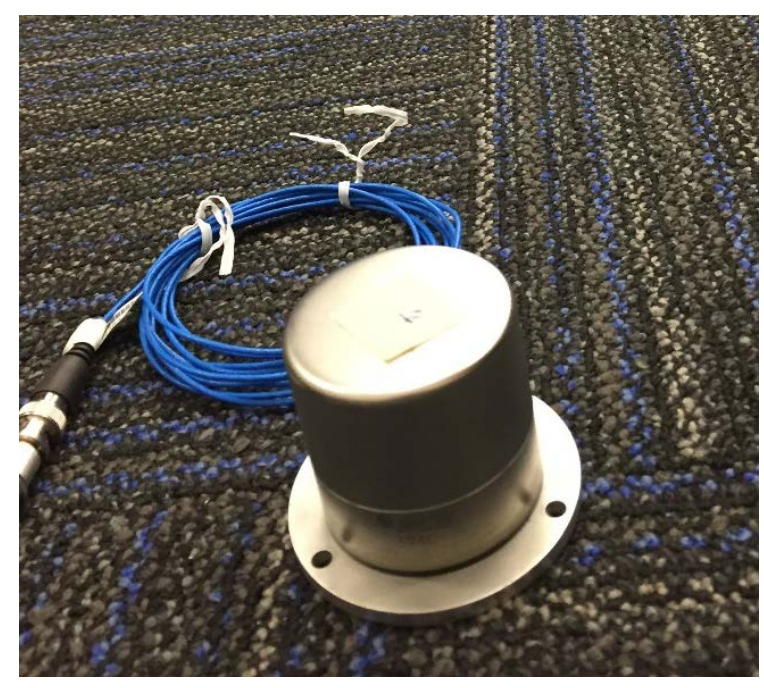

Figure 3.5: 393C PCB Piezotronics

Figure 3.6 illustrates the connection between different equipment. 


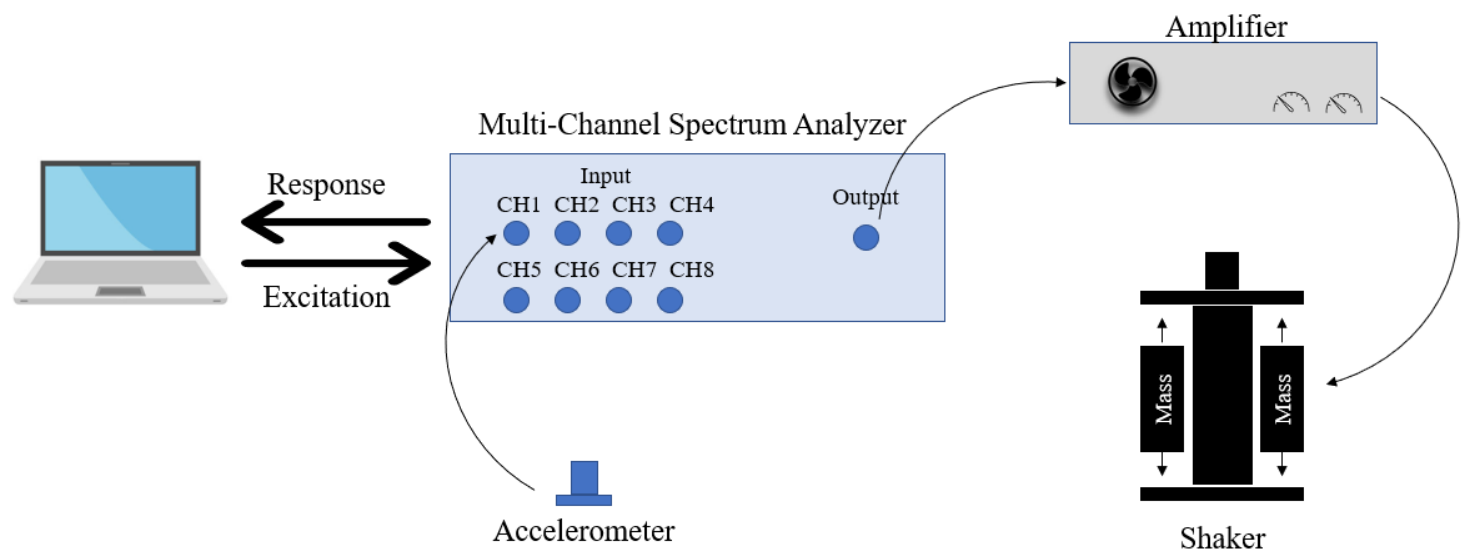

Figure 3.6: Equipment Setup

\subsection{Determination of Frequency Response Function}

This section describes the frequency response function (FRF) of this bay to characterize its response with respect to different frequencies. Figure 3.7 shows the partition layout as well as the experiment setup. The blue lines represent the CMU walls. Two related photos are also shown in Figure 3.8. The array of accelerometers in the Xand Y-directions are shown in Figures 3.8(a) and 3.8(b), respectively.

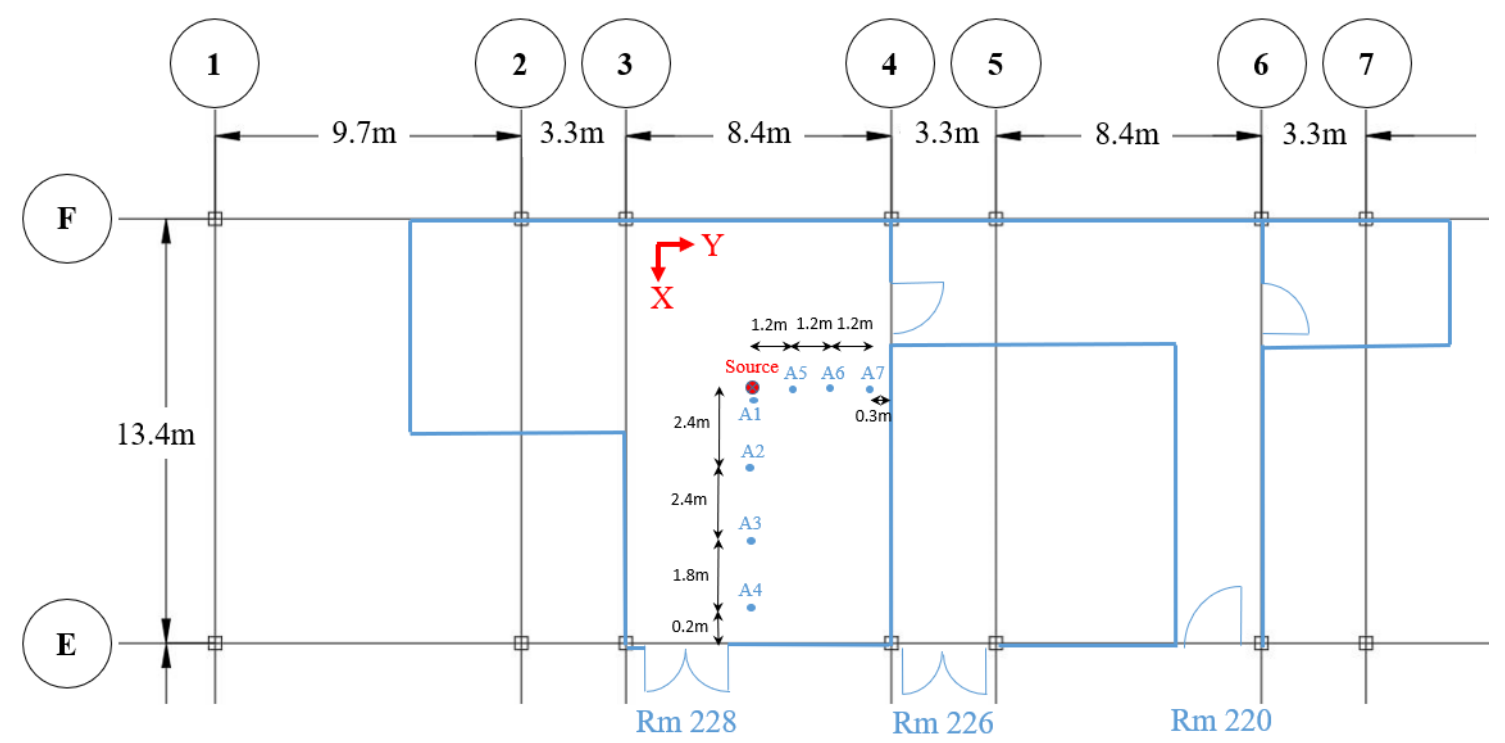

Figure 3.7: Drawing for the Tested Floor and Location of Accelerometers and the Shaker 


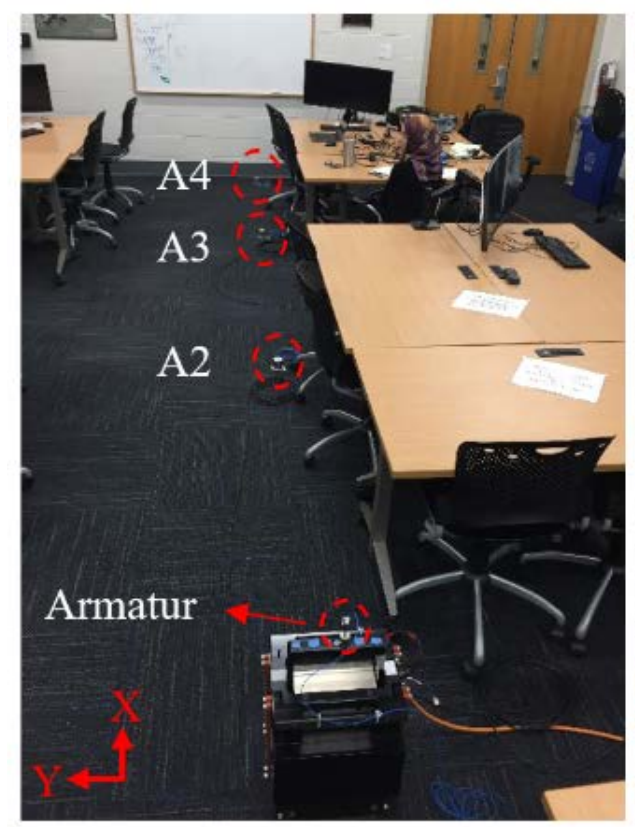

(a) X-Direction (Group 1)

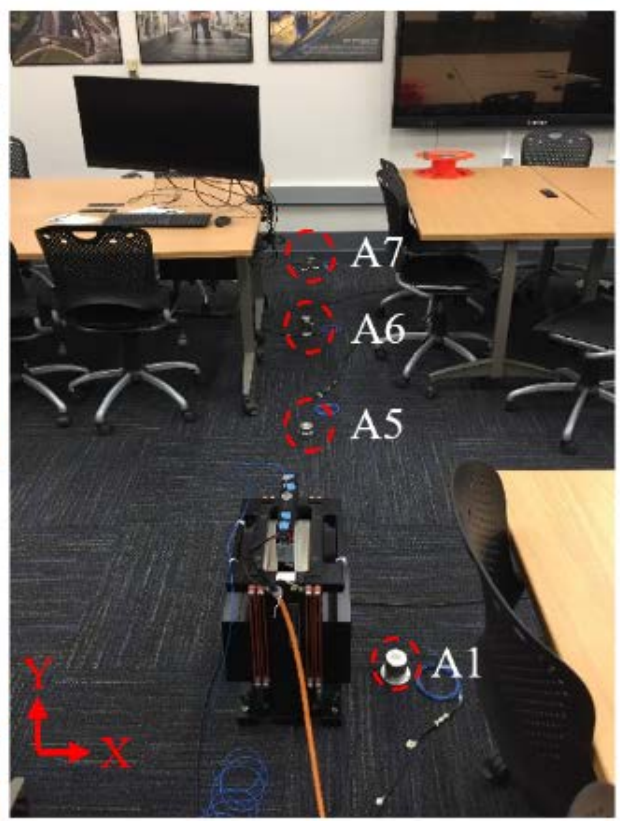

(b) Y-Direction (Group 2)

Figure 3.8: Position of Accelerometers and the Shaker

Seven accelerometers were used in this study. In Figure 3.7, A1 through A7, as shown above by the blue dots, indicate accelerometers, and the red dot is the location of the shaker as the source of vibration. The A1 accelerometer, which was placed very close to the shaker, is called a driving-point accelerometer. Assume Group 1 and Group 2 include A2-A4 accelerometers (X-direction) and A5-A7 accelerometers (Y-direction), respectively. The sampling frequency was set to 10,240 samples per second.

\subsubsection{Theory of Frequency Response Function}

As mentioned in Chapter 1, each vibration event consists of an excitation, a path, and a receiver. The goal of FRF computation is to investigate how a path, in this case the tested concrete floor, reacts to the different frequencies. For this purpose, an excitation signal (input) is applied to the path, and the response of the system (output) is simultaneously acquired. Simply, FRF is the ratio of the output (response) signals of a structure to the input (excitation) signal. The first step to computing the FRF is to convert 
the time-domain input and output signal into the frequency-domain using the fast Fourier transform, as shown in Equation 3.1:

$$
\hat{Y}(f)=\int_{-\infty}^{\infty} y(t) e^{-i .2 \pi \cdot f . t} d t
$$

where ^sign represents the frequency-domain signal, $f$ is the frequency bins, and $y(t)$ can be any arbitrary time-domain signal. Next, the cross-spectrum between the frequency-domain excitation and response (Equation 3.2) and the auto-spectrum of the excitation force (Equation 3.3) and the auto-spectrum response (Equation 3.4) need to be computed, as shown below:

$$
\begin{gathered}
\hat{S}_{e y}=\hat{E}^{*}(f) \cdot \hat{Y}(f) \\
\hat{S}_{e e}=\hat{E}^{*}(f) \cdot \hat{Y}(f) \\
\hat{S}_{y y}=\hat{Y}^{*}(f) \cdot \hat{Y}(f)
\end{gathered}
$$

where the subscripts $e$ and $y$ represent the excitation force and response, respectively. $E$ and $Y$ also represent the excitation force and response, respectively. The FRF can then be computed using Equation 3.5, which is a function of the frequency and is designated by $H$ :

$$
H(f)=\frac{\hat{S}_{e y}}{\hat{S}_{e e}}
$$

Coherence is a value from 0.0 to 1.0 that implies how much of the output (received) signal is correlated to the input (excitation) signal, and it can be computed using Equation 3.6 below. The coherence is 1.0 when the received signal is perfectly correlated to the excitation signal with absolutely no additive noise, while a coherence of 0.0 represents no correlation whatsoever between the received signal and the excitation signal:

$$
\gamma^{2}(f)=\frac{\hat{S}_{e y}^{2}}{\hat{S}_{e e} \cdot \hat{S}_{y y}}
$$

\subsubsection{Estimation of Frequency Response Function (FRF)}

In computing the FRF plots for the tested floor, the excitation signal of the shaker is captured by an accelerometer that is mounted on the armature of the shaker. The armature weight is $2.8 \mathrm{~kg}$. In addition, $30.6 \mathrm{~kg}$ mass blocks were added to the shaker. 
Therefore, the shaker applied up to approximately $98 \mathrm{~N}$ force on the floor in this case. The chosen excitation signal for this purpose is white noise in the frequency range of $5 \mathrm{~Hz}$ to $200 \mathrm{~Hz}$. The white noise excitation signal is a kind of random signal that excites all frequencies with almost constant power spectral density in the chosen frequency range. This study uses this frequency range to generate the white noise excitation because the minimum and maximum operating frequency limits of the shaker are $5 \mathrm{~Hz}$ and $200 \mathrm{~Hz}$, respectively. It should be noted that the tests were conducted after hours, overnight, or on weekends to minimize extraneous noises. The duration of data acquisition was $50 \mathrm{sec}$, which was broken down into 10 sec at the beginning and end of each test - when the shaker was not operating - and $30 \mathrm{sec}$ in between - when the shaker was generating white noise. Figures 3.9 and 3.10 show the waveform and spectrum of the excitation and received signals, respectively. Notice that the $\mathrm{Y}$-axis scale in these two figures is different. Obviously, the excitation signal has a higher amplitude (Figure 3.9) than the received signal (Figure 3.10). Note that the weight of the armature in the shaker was $33.4 \mathrm{~kg}$. When developing the FRF plots, only 26 seconds (the time duration from 12 to 38 seconds) was considered. This is the time when the shaker was generating vibration. This duration of the signal is divided into thirteen blocks, and each block is multiplied by the Hanning window (Harris, 1978). The average of these blocks reduces the effect of leakage in the spectrum. Leakage can distort the transformation of the time-domain data into the frequency-domain data, resulting in frequency-domain data that is not the proper representation of the timedomain data (Avitabile, 2001). Moreover, the division of the time of interest into thirteen blocks is meant to achieve a smoother spectrum and reduce the effect of noise; therefore, the length of each time block is two seconds, and the frequency resolution is $0.5 \mathrm{~Hz}$. The spectrum shown in Figure 3.9 and 3.10 corresponds to the first block only. 


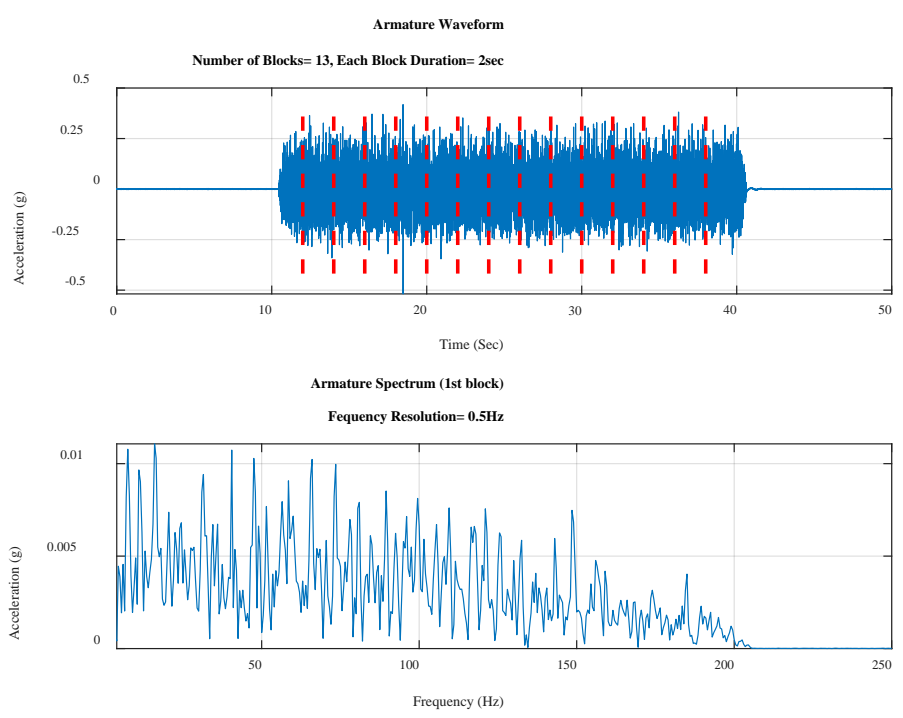

Figure 3.9: Time-Domain and Corresponding Frequency-Domain of White Noise Vertical Excitation Signal
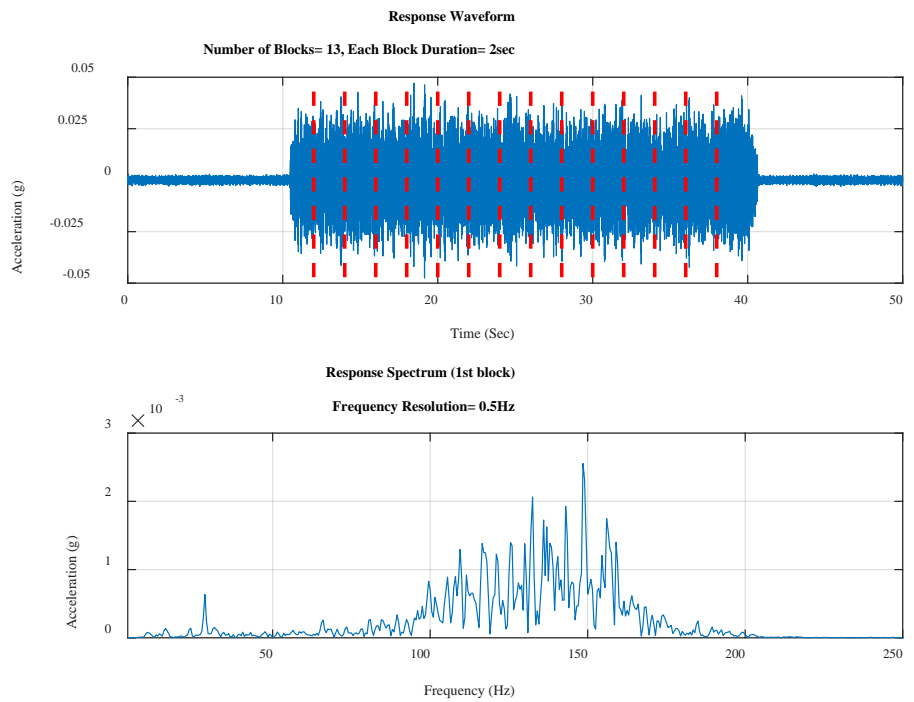

Figure 3.10: Time-Domain and Corresponding Frequency-Domain of A1 Response Due to the White Noise Vertical Excitation Signal

It should be mentioned that only up to $250 \mathrm{~Hz}$ of the spectrum was shown in Figure 3.9 and 3.10. It is worth noting that the maximum frequency, $f_{\max }$, minimum frequency, $f_{\min }$, and frequency resolution, $\Delta f$, can be computed from the following equations:

$$
f_{\max }=F_{S}
$$




$$
f_{\text {min }}=\frac{F_{s}}{n f f t}=\Delta f
$$

where $F_{s}$ is the sampling frequency, and $n f f t$ is the number of FFT points. Figures 3.11(a) and 3.11(b) show the FRF and coherence for all the accelerometers in the X-and Y-directions separately.

Based on the FRF plots, the bay is more responsive for frequencies between 80 to $180 \mathrm{~Hz}$ in both Group 1 and Group 2 accelerometers, and the coherence of this frequency range is higher than $80 \%$, demonstrating that the received data in this range are higher correlated to the excitation signal. Relatively lower coherence in the A4 and A7 accelerometers is due to these two accelerometers being close to the girder and CMU walls located on column lines E and 4, respectively (see Figure 3.7). Thus, they reported a lower response than the other accelerometers. This may be because girders and CMU walls are very stiff, and the vibration sensors close to such areas reflect a relatively lower energy vibration signal.
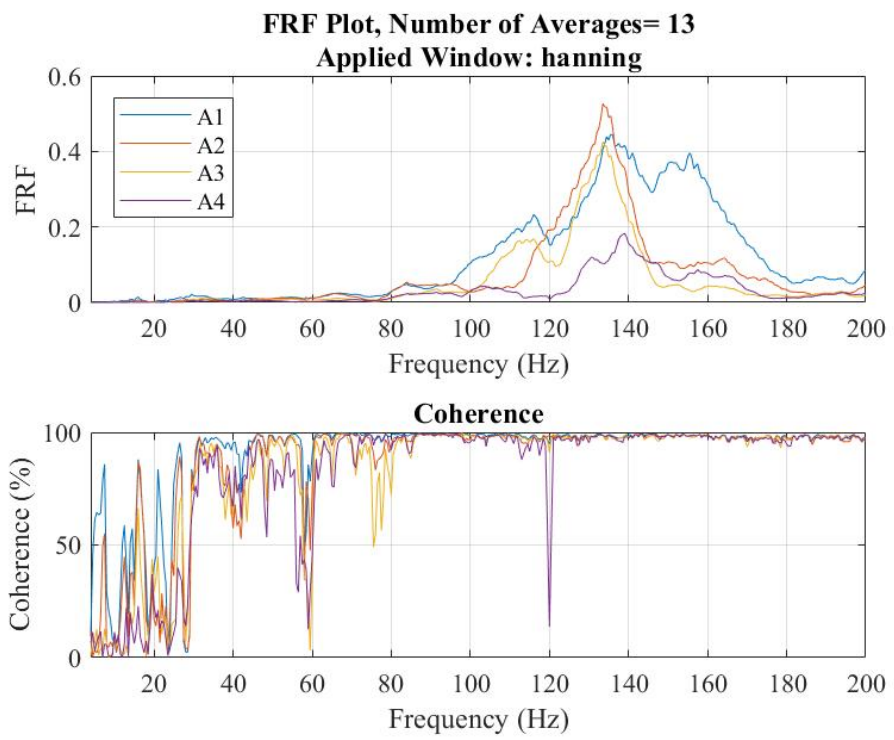

(a) Accelerometers in X-Direction 

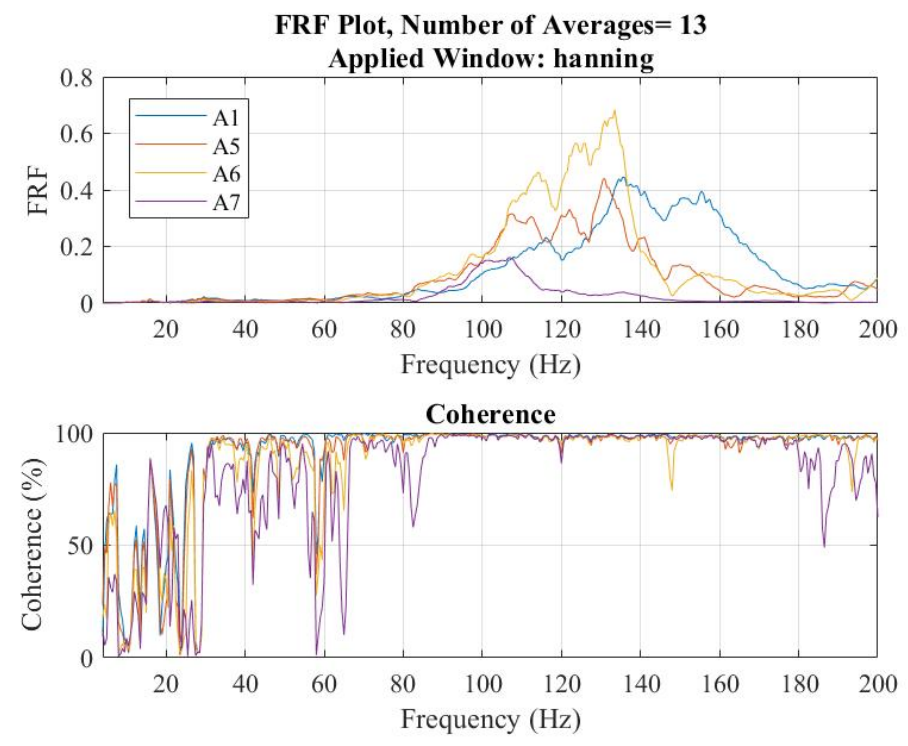

(b) Accelerometers in Y-Direction

Figure 3.11: FRF and Coherence for Set of Accelerometers in X-and Y-Direction

\subsection{Estimation of Wave Propagation Speed (WPS)}

As already mentioned, the time delay is needed for the computation of wave propagation speed (WPS). Sections 3.5.1 and 3.5.2 describe the computation of time delay and wave propagation speed using the time-domain approach and frequency-domain approach described in Chapter 2. Figure 3.12 illustrates the organization of this section.

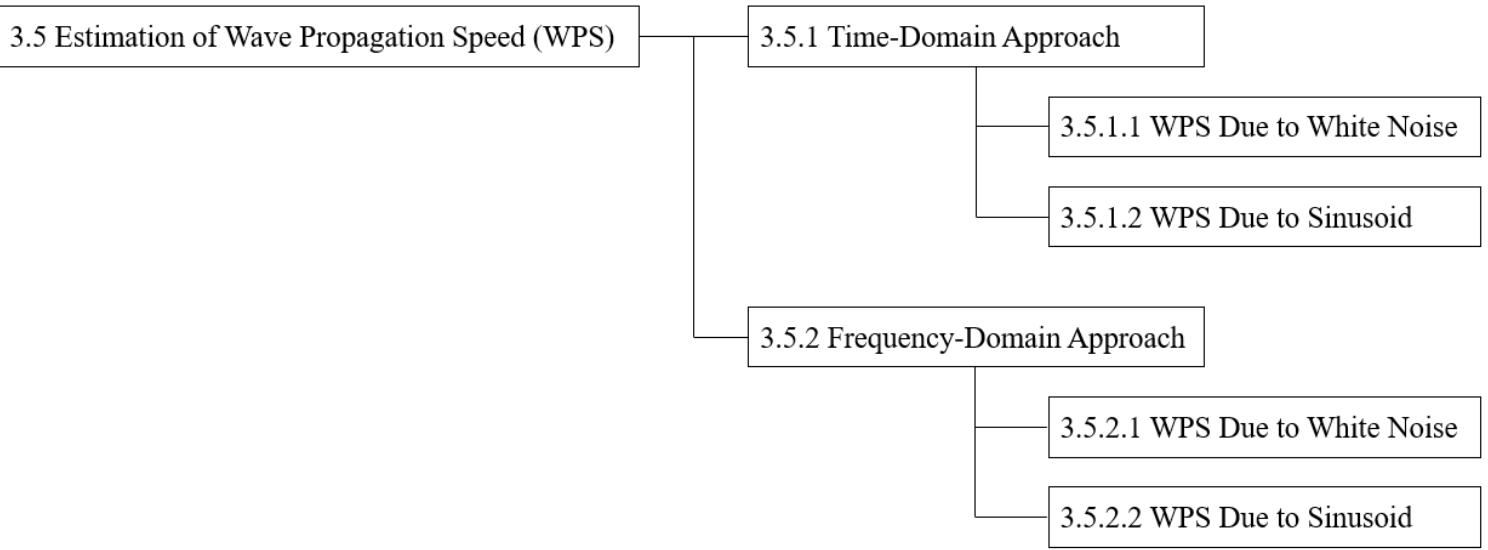

Figure 3.12: Organization of Section 3.5

Figure 3.13 shows the test setups for the shaker and all accelerometers used for the estimation of WPS, which is the same configuration shown in Figure 3.7. 


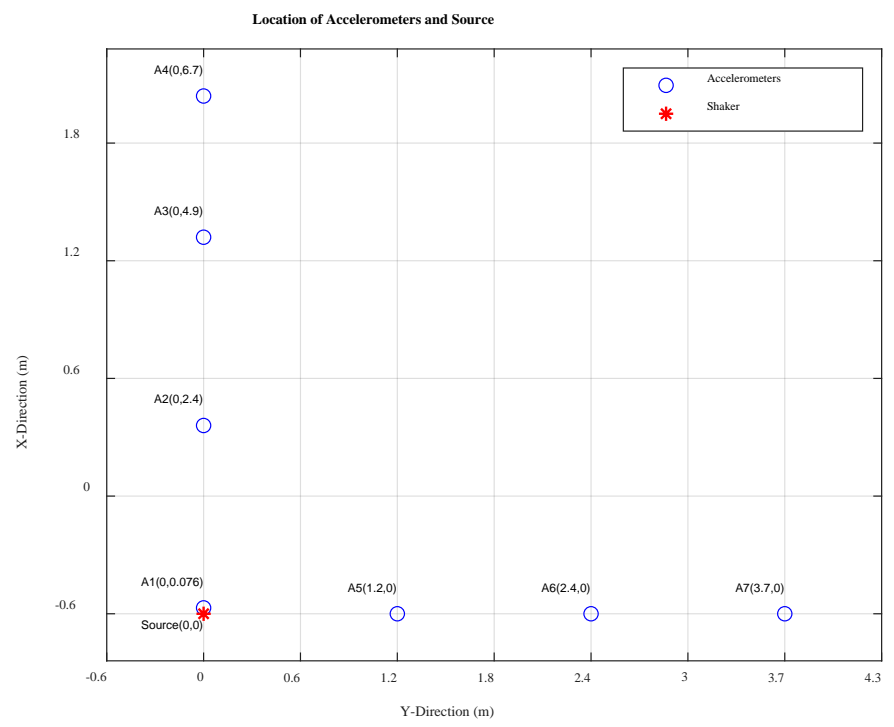

Figure 3.13: Configuration of the Shaker and Accelerometers for WPS Estimation

\subsubsection{Time-Domain Approach}

In the time-domain approach, signal post-processing is utilized in the time-domain to estimate the time delay, as explained in Section 2.3.2.

\subsubsection{WPS Due to White Noise}

White noise with a frequency range of $5 \mathrm{~Hz}$ to $200 \mathrm{~Hz}$ was used in each test. For this frequency range, two separate tests were conducted with two different powers for the shaker to investigate the effect of excitation strength on the estimation of speed propagation. The following table shows the list of tests:

Table 3.2: Name of Experiments

\begin{tabular}{|c|c|c|c|}
\hline Name & $\begin{array}{c}\text { Excitation } \\
\text { Signal }\end{array}$ & $\begin{array}{c}\text { Frequency } \\
\text { Range }\end{array}$ & Power of Shaker \\
\hline Speed25 & White Noise & $5-200 \mathrm{~Hz}$ & $50 \%$ \\
\hline Speed26 & White Noise & $5-200 \mathrm{~Hz}$ & $25 \%$ \\
\hline
\end{tabular}

Attempts were made to keep the vibration level within the elastic behavior of the concrete floor. The reason for not using more than $50 \%$ of the shaker power was because such a high vibration level may bring the dynamic property of materials into the nonlinear 
range. Also, power less than $25 \%$ was avoided because it could cause farther away accelerometers to receive a weak response, and such a low level of vibration would technically not cause any discernable issues. Figure 3.14 shows the excitation signal for tests Speed25 and Speed26. Notice that the Y-axis scale shown in Figures 3.14(a) and 3.14(b) is different. Clearly, Figure 3.14(a), in which the power of the shaker is at 50\%, has a higher amplitude. The other criterion used in these figures to show the power of each signal is the root mean square (RMS) for the entire signal. As shown in Figure 3.14, the RMS of Speed25 is higher than that of Speed26. Both accelerations reported in Figure 3.14 were from the accelerometer mounted on the armature of the shaker.

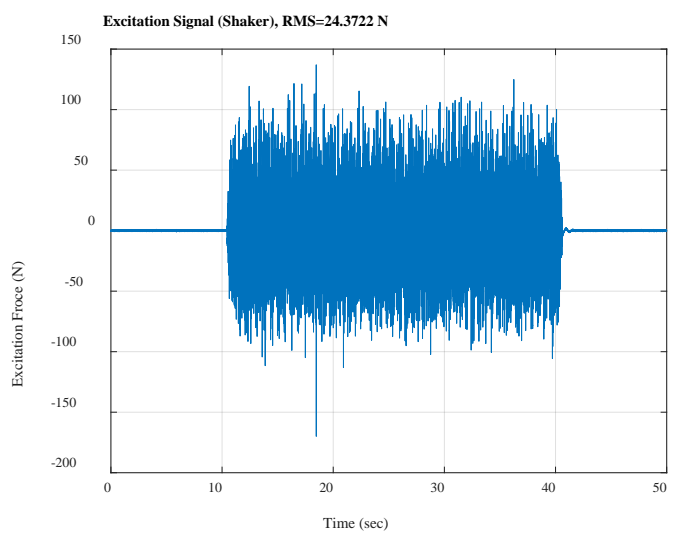

(a) Test Speed25

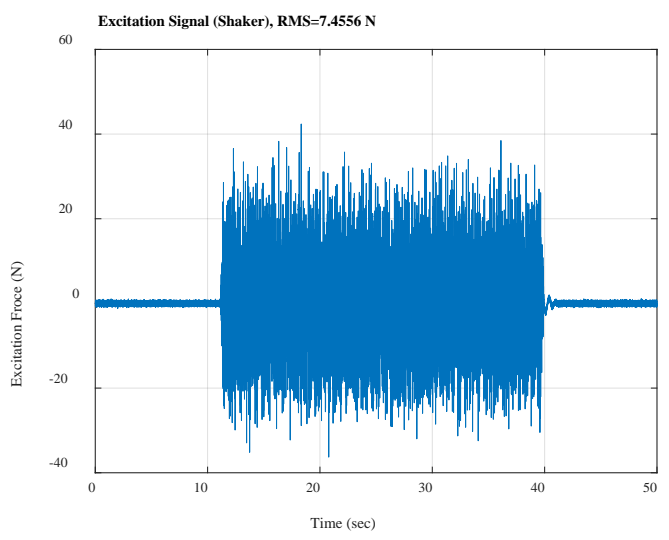

(b) Test Speed26

Figure 3.14: Excitation Signal

Figure 3.15 shows the received signals for the test Speed 25 with $50 \%$ of the shaker power being used. All plots in this figure have the same Y-axis scale for easier comparison. Regarding Figure 3.7, accelerometers A4 and A7 are closer to the CMU wall and girder on column line E and 4, respectively, compared to other accelerometers. Their distances to the wall are $0.28 \mathrm{~m}$ and $0.33 \mathrm{~m}$, respectively. This justifies the weak response for these two accelerometers. As explained earlier, the coherence of these two accelerometers is also relatively less than that of the other accelerometers. 
Received Acceleration Signals
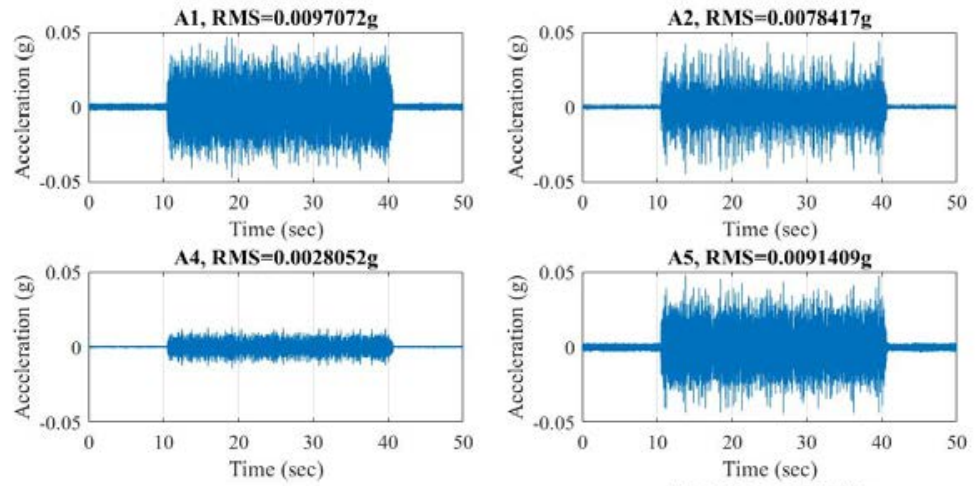

A5, RMS $=0.0091409 \mathrm{~g}$
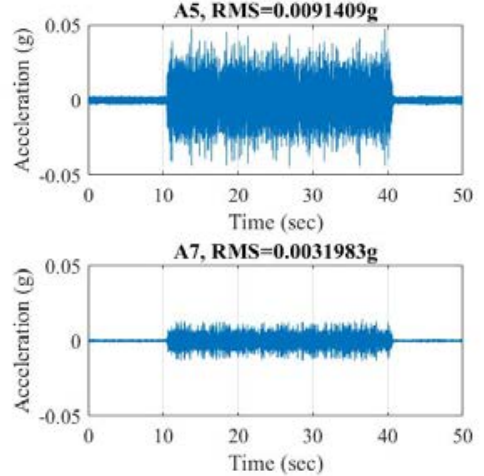
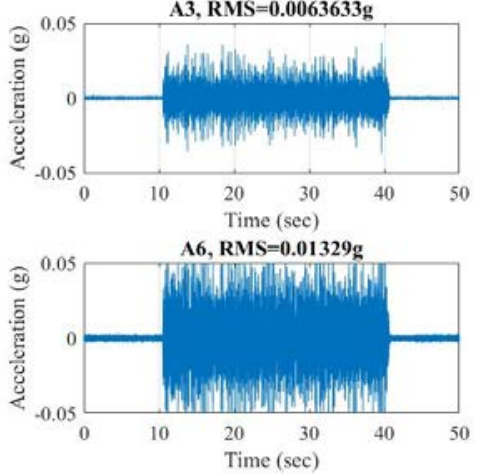

Figure 3.15: Received Signals for Test Speed25

Figure 3.16 shows the power spectral density (PSD) of both the excitation signal and the A2 accelerometer in a logarithmic scale for the test Speed25. The sampling frequency rate was set to 10,240 samples per second. The window used in this figure is the hamming window, and the length of each window is 0.5 seconds, which is equivalent to 5,120 samples, with an overlap of $75 \%$. The number of frequency points, $n f f t$, was twice as high as the number of window samples, which is 10,240 samples, giving the frequency resolution of $1 \mathrm{~Hz}$. 

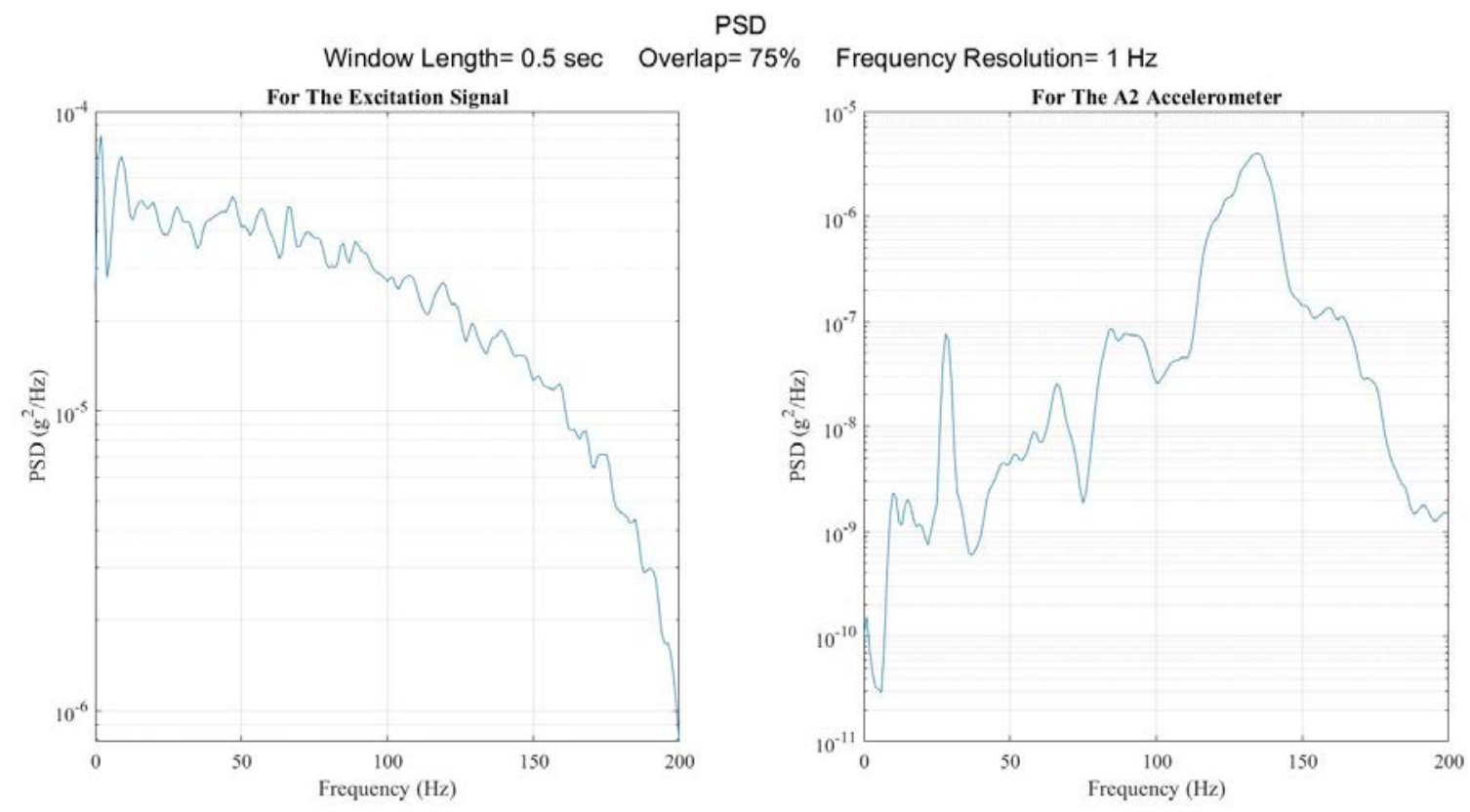

Figure 3.16: PSD of Excitation and A2 Accelerometer for the Test Speed25

The cross-correlation is used to find the time delay between the source and each accelerometer. The details for the computation of cross-correlation were explained in Section 2.3.2. Cross-correlation works fairly well in low noise environments (Ianniello, 1982; Champagne et al., 1996). The concrete floor is a sophisticated environment, and attempts were made to isolate the main source signal and suppress the noise power. Therefore, four different cases for data post-processing were considered to investigate the possibility of noise being included in the acquired data.

- Case 1: Entire signal with no manipulation.

- Case 2: Entire signal + filtered in the frequency range of interest.

- Case 3: Signal gated in time of interest + filtered in the frequency range of interest.

- Case 4: Signal gated in time of interest + filtered in the frequency range of interest + partial whitening.

Figure 3.17 shows the order of processing techniques available in each case as a flowchart. Each processing technique will be discussed separately. 

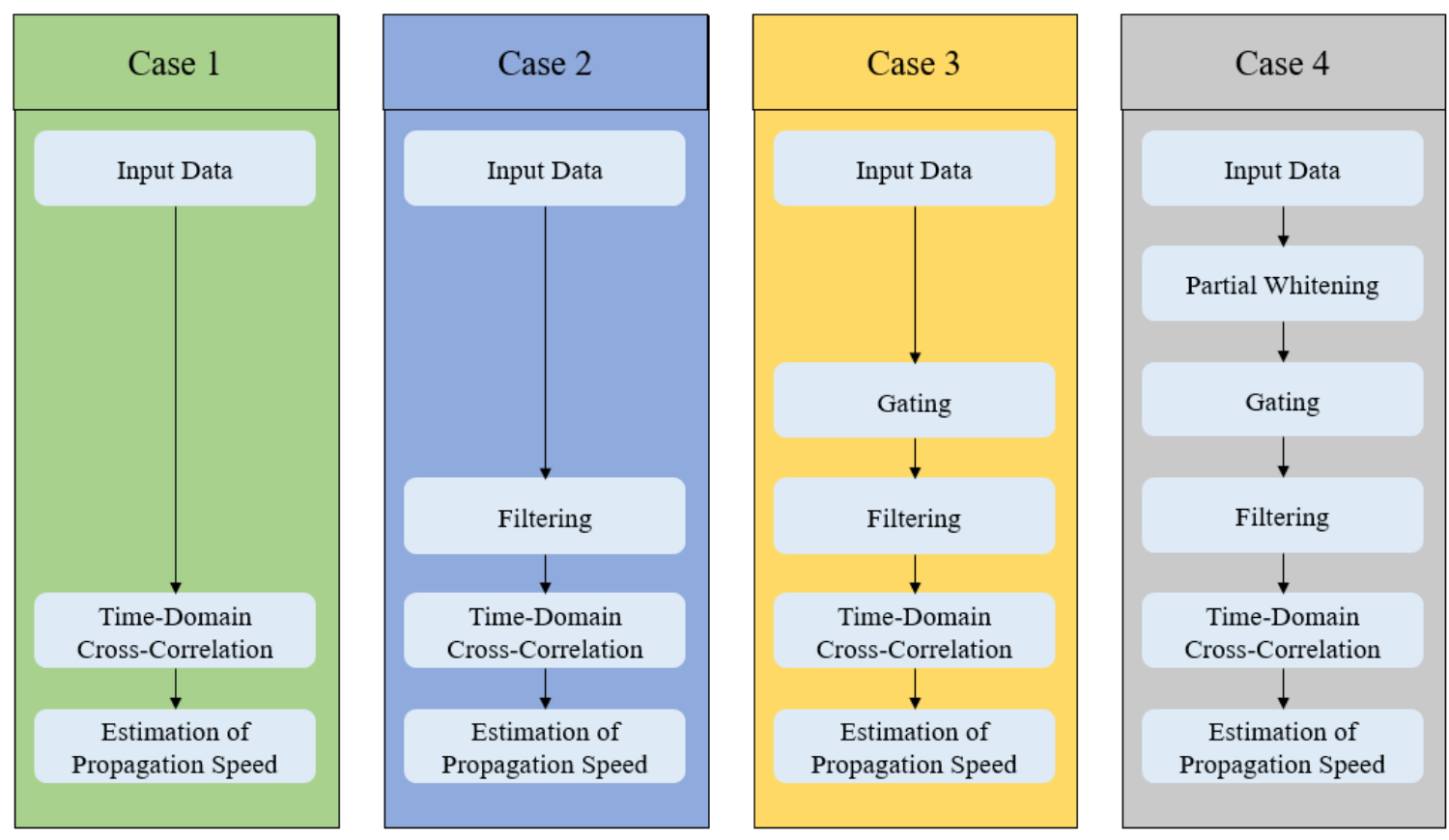

Figure 3.17: Order of Processing Techniques Available in Each Case

\section{Case 1: Entire Signal with No Manipulation}

In this case, the cross-correlation function between the excitation signal and each accelerometer is applied in the time-domain to estimate the time delay using the entire signal with no manipulation. The number of samples in each signal is equal to the sampling frequency multiplied by the acquisition time (50 sec), resulting in 512,000 samples. The number of samples in the cross-correlation function is the sum of the number of samples in the two input signals. As an example, the cross-correlation between the excitation signal and the A2 accelerometer is shown in Figure 3.18. The single clear peak with a high amplitude shows the similarity between the excitation signal and the A2 accelerometer at a lag corresponding to the time delay. The similarity elsewhere is considerably smaller. 


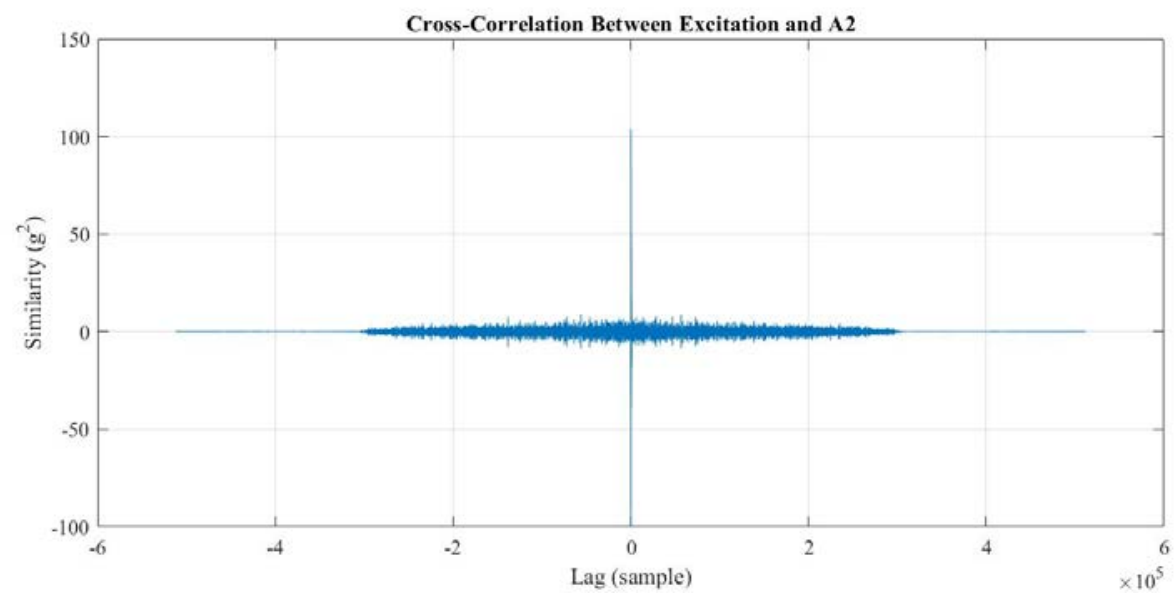

Figure 3.18: Cross-Correlation between Excitation and A2 Accelerometer

Figure 3.19 is a magnified view of Figure 3.18 near zero. Only peaks after the zerolag are considered because all accelerometers received the excitation signal with a delay after the vibration excitation was propagated. In the cross-correlation, positive peaks indicate the similarities when the two signals are in phase and negative peaks when the two signals are 180 degrees out of phase. Only positive peaks are considered in this research. In Figure 3.19, the X-axis represents the lag, and the unit of the lag is sample. Time delay, $T D_{t}$, can be computed from Equation 2.15. Subscript $t$ expresses the use of the time-domain approach. The distance between each accelerometer and the excitation source is known. For example, the distance between the source and A2 accelerometer is $2.4 \mathrm{~m}$. The time delay and estimated speed corresponding to peak \#1 (indicating the highest similarity in Figure 3.19) are 0.024 seconds and $102 \mathrm{~m} / \mathrm{s}$, respectively. 


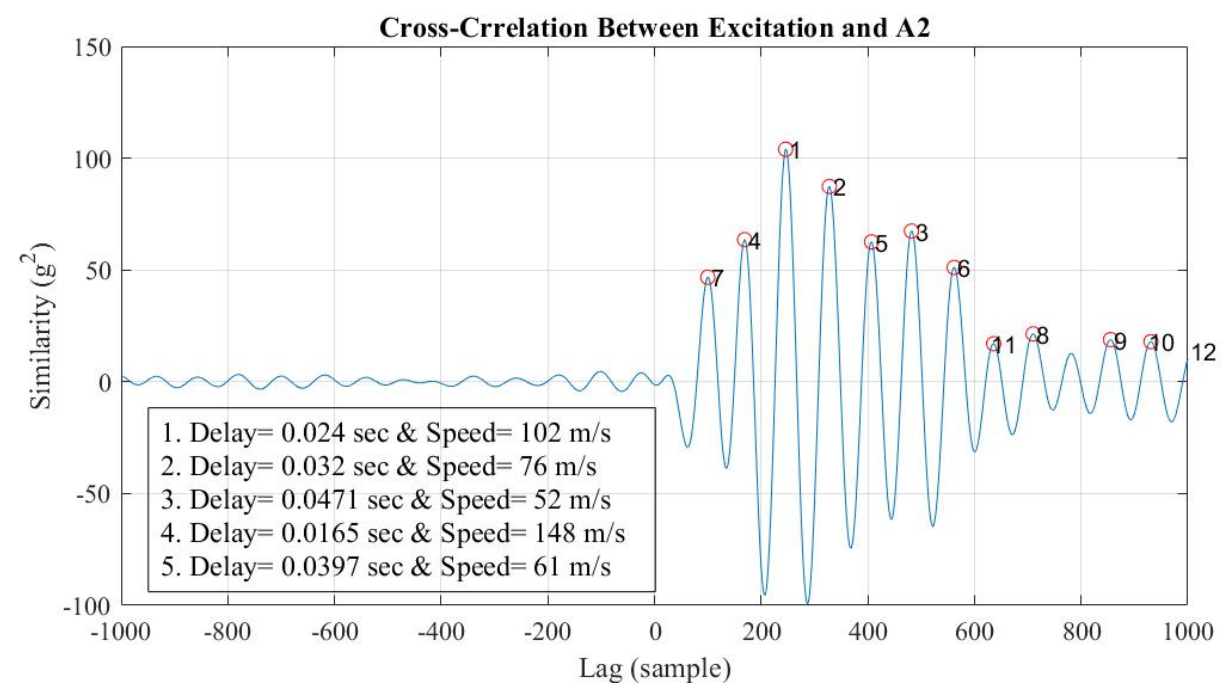

Figure 3.19: Close View of Cross-Correlation between Excitation and A2 Accelerometer

Two points need to be made here. First, notice that the consecutive peaks are very close to each other in time. Every 1024 samples in the cross-correlation correspond only to a 0.1-second delay. Second, sources of error like attenuation, dispersion, and multipath effects can distort the shape of the received signals and, therefore, degrade computation accuracy. Accordingly, the lag corresponding to the maximum positive peak may not represent the correct time delay. Also, the greater the distance between the source of vibration and the receiver, the more possibility of distortion. Compared to Figure 3.19, Figure 3.20 shows the cross-correlation between the excitation signal and A3 accelerometer, which is farther away than the A2 accelerometer. The estimated speed of the lag corresponding to peak \#1 (maximum similarity) for the A3 accelerometer in Figure 3.20 is $168 \mathrm{~m} / \mathrm{s}$, whereas the estimated speed for peak \#1 (maximum similarity) is $102 \mathrm{~m} / \mathrm{s}$ for the A2 accelerometer in Figure 3.19. This inconsistency between the two accelerometers may be due to existing distortion in the signals. Other positive peaks after the zero-lag were also examined. In this section, the results for the first ten peaks will be investigated for all cases. 


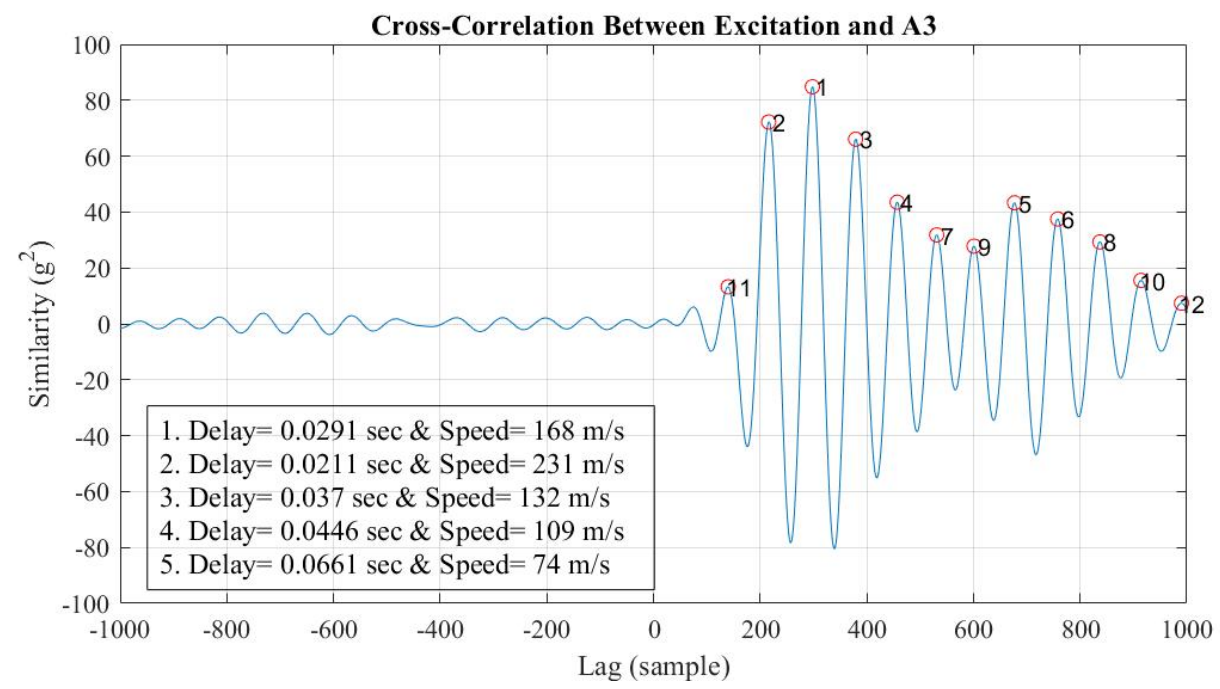

Figure 3.20: Close View of Cross-Correlation between Excitation and A3 Accelerometer

Table 3.3 shows the estimated WPS between each accelerometer and the excitation signal based on the first ten highest peaks in the cross-correlation. The second column shows the pair of accelerometers used in the cross-correlation function. "Arm” stands for armature, which indicates the accelerometer that is mounted on the armature of the shaker reporting the excitation signal. The A1 accelerometer was very close to the shaker, and the correlation between A1 and Arm was very poor (Barrett 2006). Therefore, it will not be considered in this dissertation. The nature of these results may be due to the close sourcesensor distance, which likely caused the signal to be dominated by high-frequency components (Bahroun et al. 2014). In Table 3.3, accelerometers A2, A3, and A4 are in the X-direction, and accelerometers A5, A6, and A7 are in the Y-direction. As already mentioned, the estimated WPS corresponds to one of the peaks in the cross-correlation. Therefore, the first estimated WPS (from left to right) that are close together for all accelerometers in each direction are highlighted in Table 3.3. These highlighted numbers were considered as the correct estimated WPS in that direction. The average of these numbers is shown in the last column. 
Table 3.3: Estimated Propagation Speed (Case 1: Entire Signal with No Manipulation)

\begin{tabular}{|c|c|c|c|c|c|c|c|c|c|c|c|c|c|}
\hline \multirow{2}{*}{ Direction } & \multirow{2}{*}{$\begin{array}{c}\text { Cross- } \\
\text { correlation }\end{array}$} & \multirow{2}{*}{ Distance } & \multicolumn{10}{|c|}{ Estimated Speed $(\mathrm{m} / \mathrm{s})$ for each Peak in the Cross-Correlation } & \multirow{2}{*}{$\begin{array}{c}\text { Avg of } \\
\text { Highlights }\end{array}$} \\
\hline & & & 1st & 2nd & 3rd & 4th & 5th & 6th & 7th & 8th & 9th & 10th & \\
\hline \multirow{3}{*}{$\mathrm{X}$} & Arm - A2 & 2.44 & 102 & 76.1 & 51.8 & 148 & 61.3 & 44.4 & 250 & 35.2 & 29.2 & 26.8 & \multirow{3}{*}{107.9} \\
\hline & Arm - A3 & 4.88 & 168 & 231 & 132 & 109 & 73.8 & 65.9 & 94 & 59.7 & 83.1 & 54.6 & \\
\hline & Arm - A4 & 6.71 & 290 & 184 & 152 & 224 & 128 & 113 & 427 & 83 & 91.8 & 101 & \\
\hline \multirow{3}{*}{$\mathrm{Y}$} & Arm - A5 & 1.22 & 78 & 169 & 51.2 & 37.9 & 22.9 & 19.9 & 12.3 & 11.4 & 10.6 & 13.5 & \multirow{3}{*}{74.4} \\
\hline & Arm - A6 & 2.44 & 140 & 94.9 & 271 & 72.2 & 58.9 & 38.7 & 50.1 & 34.3 & 44 & 22.6 & \\
\hline & Arm - A7 & 3.66 & 174 & 119 & 310 & 90.3 & 72.9 & 60.9 & 52.5 & 46.5 & 41.7 & 24.2 & \\
\hline
\end{tabular}

\section{Case 2: Entire Signal + Filtered in Frequency Range of Interest}

This case is considered to investigate the influence of filtering on the results. As mentioned in Section 3.4.2, the coherence is more than $80 \%$ for frequencies from 80 to $180 \mathrm{~Hz}$. In Case 2, post-processing is performed the same as Case 1, but the signal is prefiltered in the frequency range of $80 \mathrm{~Hz}$ to $180 \mathrm{~Hz}$, where the frequency components are more correlated to the excitation vibration prior to computation of the cross-correlation. Pre-filtering is applied in the frequency-domain by use of the Butterworth IIR filter with an order of three. The A2 accelerometer signal before and after filtering is shown in Figure 3.21:
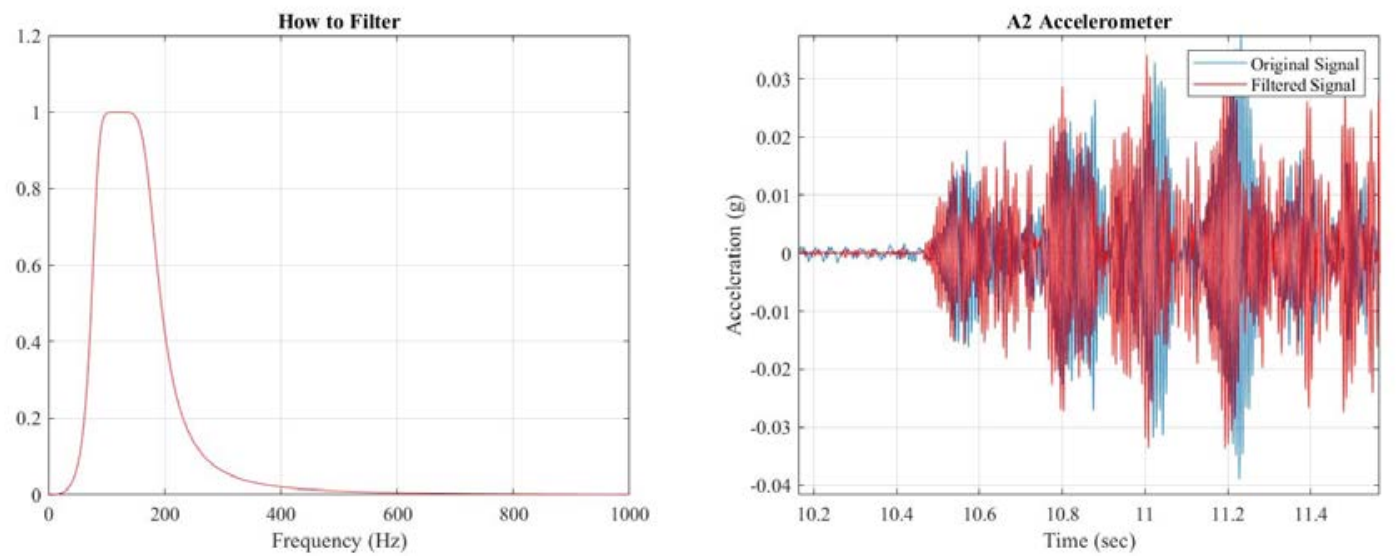

Figure 3.21: Applied Filter in Frequency-Domain and A2 Accelerometer Before and After Filtering

Table 3.4 shows the estimated propagation speed for Case 2. The results are very similar to Case 1 in both directions. 
Table 3.4: Estimated Propagation Speed (Case 2: Entire Signal + Filtered in Frequency Range of Interest)

\begin{tabular}{|c|c|c|c|c|c|c|c|c|c|c|c|c|c|}
\hline \multirow{2}{*}{ Direction } & \multirow{2}{*}{$\begin{array}{c}\text { Cross- } \\
\text { Correlation }\end{array}$} & \multirow{2}{*}{ Distance } & \multicolumn{10}{|c|}{ Estimated Speed (m/s) for each Peak in Cross-Correlation } & \multirow{2}{*}{$\begin{array}{c}\text { Avg of } \\
\text { Highlights }\end{array}$} \\
\hline & & & 1st & 2nd & 3rd & 4th & 5th & 6th & 7th & 8th & 9th & 10th & \\
\hline \multirow{3}{*}{$\mathrm{X}$} & Arm - A2 & 2.44 & 101 & 76.4 & 61.3 & 148 & 51.7 & 44.5 & 260 & 39.1 & 24.9 & 27 & \multirow{3}{*}{107.6} \\
\hline & Arm - A3 & 4.88 & 168 & 132 & 231 & 109 & 65.9 & 73.8 & 93.9 & 83.1 & 59.5 & 367 & \\
\hline & Arm - A4 & 6.71 & 151 & 183 & 291 & 129 & 224 & 429 & 113 & 83.3 & 91.8 & 101 & \\
\hline \multirow{3}{*}{$\mathrm{Y}$} & Arm - A5 & 1.22 & 78.5 & 169 & 51.2 & 37.9 & 11.4 & 12.4 & 13.6 & 15.1 & 3.3 & 6.4 & \multirow{3}{*}{74.7} \\
\hline & Arm - A6 & 2.44 & 141 & 95.3 & 72.2 & 271 & 58.8 & 24.6 & 22.7 & 15.8 & 38.6 & 26.9 & \\
\hline & Arm - A7 & 3.66 & 173 & 120 & 302 & 90.7 & 73.4 & 1040 & 61.5 & 10 & 18.6 & 42.3 & \\
\hline
\end{tabular}

\section{Case 3: Signal Gated in Time of Interest + Filtered in the Frequency of Interest}

In contrast with Case 2, this case examines the influence of only a small portion of the signal in the post-processing stage, which allows for two benefits. First, the processing is much faster due to having to consider a shorter length of time than the entire 50 seconds. As already noted, each second contains 10,240 sample data. Second, it gives the option to pick any part of the signal that is of interest by the researcher. For example, due to multipath effects, the part of the signal closer to the end of data acquisition may include more distortions that can degrade the accuracy of the computation, which would make it a bad choice for this investigation. Conversely, the beginning portion of the signal may give more accurate results and would therefore be worth examining. Figure 3.22 shows the twosecond period of interest - beginning immediately before when the shaker started working - in red for the A2 accelerometer. Table 3.5 shows the results for Case 3, which are very analogous to Cases 1 and 2 . It should also be observed that a study conducted by the researcher to consider different window lengths in a different part of the signal has provided almost the same results. 


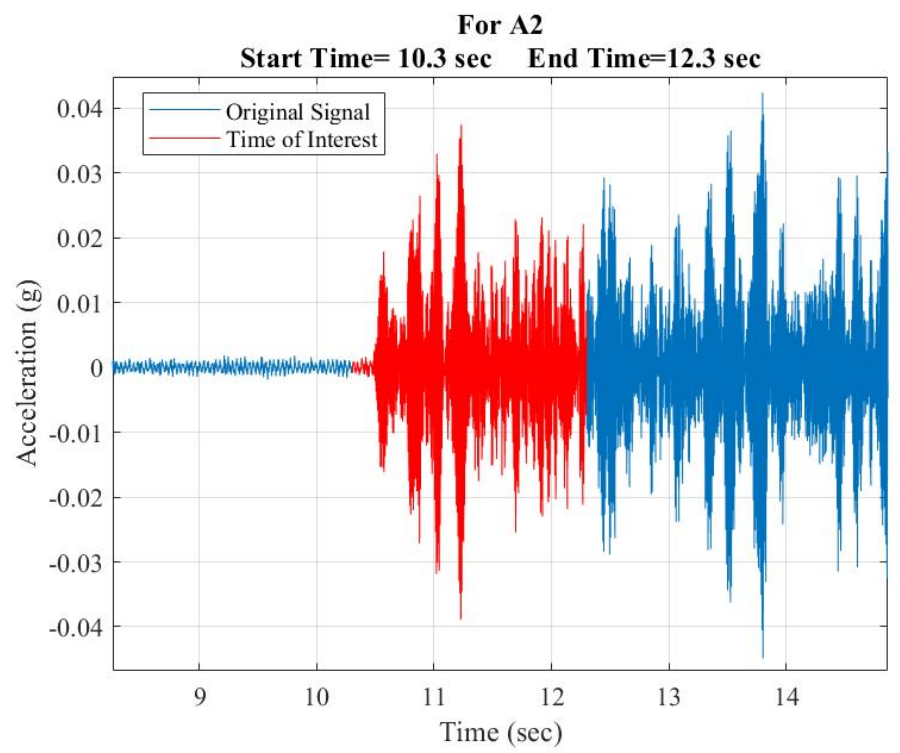

Figure 3.22: Time of Interest in Case 3

Table 3.5: Estimated Propagation Speed (Case 3: Signal Gated in Time of Interest + Filtered in Frequency Range of Interest)

\begin{tabular}{|c|c|c|c|c|c|c|c|c|c|c|c|c|c|}
\hline \multirow{2}{*}{ Direction } & \multirow{2}{*}{$\begin{array}{c}\text { Cross- } \\
\text { Correlation }\end{array}$} & \multirow{2}{*}{ Distance } & \multicolumn{10}{|c|}{ Estimated Speed (m/s) for each Peak in Cross-Correlation } & \multirow{2}{*}{$\begin{array}{c}\text { Avg of } \\
\text { Highlights }\end{array}$} \\
\hline & & & 1st & 2nd & 3rd & 4th & 5th & 6th & 7th & 8th & 9th & 10th & \\
\hline \multirow{3}{*}{$\mathrm{X}$} & Arm - A2 & 2.44 & 101 & 76.4 & 61.3 & 148 & 51.7 & 44.5 & 260 & 39.1 & 24.9 & 27 & \multirow{3}{*}{107.6} \\
\hline & Arm - A3 & 4.88 & 168 & 132 & 231 & 109 & 65.9 & 73.8 & 93.9 & 83.1 & 59.5 & 367 & \\
\hline & Arm - A4 & 6.71 & 151 & 183 & 291 & 129 & 224 & 429 & 113 & 83.3 & 91.8 & 101 & \\
\hline \multirow{3}{*}{$\mathrm{Y}$} & Arm - A5 & 1.22 & 78.5 & 169 & 51.2 & 37.9 & 11.4 & 12.4 & 13.6 & 15.1 & 3.3 & 6.4 & \multirow{3}{*}{74.7} \\
\hline & Arm - A6 & 2.44 & 141 & 95.3 & 72.2 & 271 & 58.8 & 24.6 & 22.7 & 15.8 & 38.6 & 26.9 & \\
\hline & Arm - A7 & 3.66 & 173 & 120 & 302 & 90.7 & 73.4 & 1040 & 61.5 & 10 & 18.6 & 42.3 & \\
\hline
\end{tabular}

\section{Case 4: Signal Gated in Time of Interest + Filtered in the Frequency of Interest}

\section{+ Partial Whitening}

As previously mentioned, there are two types of noise that may exist in the signal, i.e., independent and correlated noise. Reverberation is a good example of correlated noise in audio signals, which is created when the signal travels a longer distance, reflects from the boundaries, and then is received by the receiver from an indirect path. In Section 1.2.4.1, it was noted that the reflected signal is an attenuated and delayed replica of the source signal (Benesty et al. 2004). This chapter will attempt to determine if reverberation also exists in the received vibration signal. Signal pre-whitening is an additional step in processing to de-emphasize reflection by putting equal emphasis on all the frequencies in 
the signal spectrum, making it more similar to the white noise spectrum and, therefore, sharpening the cross-correlation peaks (Omologo and Svaizer, 1997). This signal processing technique has been reported to work well in reverberant environments for audio processing applications. However, the disadvantage of this method is the amplification of noise in the frequency when the noise dominates many of the frequency components. To address this problem, partial whitening is used to control the degree of whitening and thereby minimize the amount of degradation (Donohue et al. 2007). Equation 3.9 shows how pre-whitening can be applied in the frequency-domain:

$$
\hat{A}_{i}(f, \beta)=\frac{\hat{A}_{i}(f)}{\left|\hat{A}_{i}(f)\right|^{\beta}}
$$

where $\hat{A}_{i}$ is the frequency-domain of an $A_{i}$ accelerometer, $f$ represents the frequency bins, and $\beta$ is a real number between 0 and 1 . If $\beta=1$, the signal is totally whitened, whereas if $\beta=0$ there is no effect on the signal. Intermediate $\beta$ values result in partial whitening. After applying partial whitening in the frequency-domain, the signal transforms into the time-domain using the inverse fast Fourier transform, and then the cross-correlation is applied (Donohue et al., 2007). The objective of Case 4 is to investigate the effect of partial whitening on the results. Processing, in this case, is similar to Case 3, with additional partial whitening being applied. Note that partial whitening was not applied on the accelerometer mounted on the armature since it reported a clean signal that was not affected by the concrete floor. Figure 3.23 shows the effect of pre-whitening on both the time- and frequency-domains of the A2 accelerometer for $20 \%$ partial whitening. 

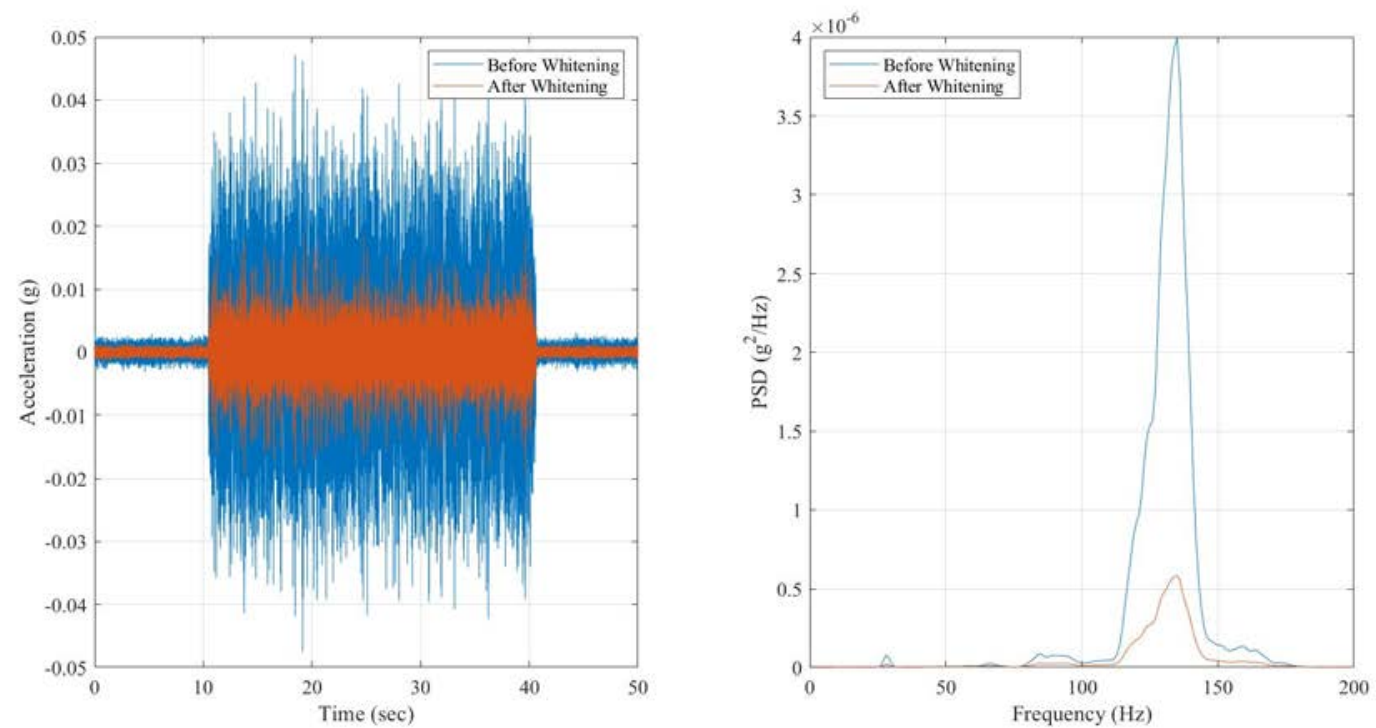

Figure 3.23: Effect of Partial Whitening on Time- and Frequency-Domain for A2 Accelerometer

Different degrees of partial whitening were tested by the researcher, and the estimated WPS results up to $60 \%$ partial whitening are close to each other within plus or minus $1.0 \mathrm{~m} / \mathrm{s}$. Table 3.6 shows the results for Case 4 for $20 \%$ partial whitening. The results are very similar to the previous cases because the whitening operation may cause little to no effect in spectral regions.

Table 3.6: Estimated Propagation Speed (Case 4: Signal Gated in Time of Interest + Filtered in Frequency Range of Interest + Partial Whitening)

\begin{tabular}{|c|c|c|c|c|c|c|c|c|c|c|c|c|c|}
\hline \multirow{2}{*}{ Direction } & \multirow{2}{*}{$\begin{array}{c}\text { Cross- } \\
\text { Correlation }\end{array}$} & \multirow{2}{*}{ Distance } & \multicolumn{10}{|c|}{ Estimated Speed $(\mathrm{m} / \mathrm{s})$ for each Peak in Cross-Correlation } & \multirow{2}{*}{$\begin{array}{c}\text { Avg of } \\
\text { Highlights }\end{array}$} \\
\hline & & & 1st & 2nd & 3rd & 4th & 5 th & 6th & 7th & 8th & 9th & 10th & \\
\hline \multirow{3}{*}{ X } & Arm - A2 & 2.44 & 102 & 76.4 & 61.3 & 148 & 51.7 & 44.4 & 257 & 6.5 & 12.2 & 39.1 & \multirow{3}{*}{107.7} \\
\hline & Arm - A3 & 4.88 & 168 & 132 & 231 & 109 & 65.9 & 73.8 & 93.9 & 59.6 & 83.1 & 33 & \\
\hline & Arm - A4 & 6.71 & 151 & 183 & 291 & 128 & 432 & 225 & 113 & 36.9 & 35.2 & 83.3 & \\
\hline \multirow{3}{*}{1} & Arm - A5 & 1.22 & 78.5 & 167 & 51.2 & 12.4 & 38.2 & 11.4 & 13.6 & 3.3 & 6.4 & 2.6 & \multirow{3}{*}{74.8} \\
\hline & Arm - A6 & 2.44 & 141 & 94.9 & 72.2 & 269 & 58.9 & 24.5 & 22.6 & 15.9 & 6.6 & 16.8 & \\
\hline & Arm - A7 & 3.66 & 174 & 300 & 120 & 90.7 & 73.6 & 936 & 18.5 & 19.5 & 61.5 & 35.2 & \\
\hline
\end{tabular}

All cases mentioned up to here were based on the test named Speed25, where the shaker power was set to $50 \%$. As has been shown, using different processing techniques to reduce the effect of any possible independent noise or correlated noise does not change the results significantly. As mentioned in Table 3.2, to investigate the effect of vibration 
level energy on the results, two different tests were conducted with the shaker power set at $50 \%$ and $25 \%$, respectively. The same procedure that was just explained for the four previous cases was also applied to the Speed 26 test in which the shaker power was set to 25\%. The results for both tests and all four cases are shown in Table 3.7 for comparison. Based on these results, it can be concluded that the level of applied vibration energy did not bring the concrete floor material into non-linear behavior. In most cases, the estimated wave propagation speeds while the shaker was working with less power are a bit higher.

Table 3.7: Propagation Speed Results for Different Shaker Power

\begin{tabular}{|c|c|c|c|c|c|c|c|c|c|}
\cline { 3 - 9 } \multicolumn{2}{c|}{} & \multicolumn{4}{c|}{ Speed25 (Shaker Power 50\%) } & \multicolumn{3}{c|}{ Speed26 (Shaker Power 25\%) } \\
\cline { 2 - 10 } \multicolumn{2}{c|}{} & Case 1 & Case 2 & Case 3 & Case 4 & Case 1 & Case 2 & Case 3 & Case 4 \\
\hline \multirow{3}{*}{ X-Dir } & Arm - A2 & 102 & 101 & 101 & 102 & 102.3 & 102.3 & 101.5 & 101.9 \\
\cline { 2 - 10 } & Arm - A3 & 109 & 109 & 109 & 109 & 110 & 110 & 109.8 & 109.8 \\
\cline { 2 - 10 } & Arm - A4 & 113 & 113 & 113 & 113 & 112.9 & 112.9 & 113.3 & 113.1 \\
\hline \multirow{3}{*}{ Y-Dir } & Arm - A5 & 78 & 78.5 & 78.5 & 78.5 & 80.5 & 80.5 & 80.5 & 80.5 \\
\cline { 2 - 10 } & Arm - A6 & 72.2 & 72.2 & 72.2 & 72.2 & 73.7 & 73.7 & 73.4 & 73.2 \\
\cline { 2 - 10 } & Arm - A7 & 72.9 & 73.4 & 73.4 & 73.6 & 74.6 & 74.6 & 74.8 & 74.8 \\
\hline
\end{tabular}

This study showed that the effect of the shaker power is negligible on the estimated WPS. The results also demonstrate that, in these two tests, the method used to estimate the vibration propagation speed was not dependent on vibration power. It was determined that the propagation speed range in the X- and Y-directions is approximately $102-113 \mathrm{~m} / \mathrm{s}$ and $72-80 \mathrm{~m} / \mathrm{s}$, respectively. These ranges will be used in Section 3.6.1 of this Chapter to locate the vibration source due to the white noise excitation.

\subsubsection{WPS Due to Sinusoid}

The excitation signal in this section is a sinusoid in each test. The list of tests is given in Table 3.8. For each frequency sinusoid, two tests with two different shaker powers were conducted to investigate the influence of excitation power on the estimated WPS. 
Table 3.8: List of Tests with Sinusoid Excitation Signals

\begin{tabular}{|c|c|c|c|}
\hline Name & Excitation Signal & $\begin{array}{c}\text { Frequency } \\
(\mathbf{H z})\end{array}$ & Power of Shaker \\
\hline Speed29 & Sinusoid & 70 & $50 \%$ \\
\hline Speed30 & Sinusoid & 70 & $75 \%$ \\
\hline Speed31 & Sinusoid & 90 & $38 \%$ \\
\hline Speed32 & Sinusoid & 90 & $50 \%$ \\
\hline Speed33 & Sinusoid & 110 & $38 \%$ \\
\hline Speed34 & Sinusoid & 110 & $50 \%$ \\
\hline
\end{tabular}

Figures 3.24 and 3.25 show the excitation signal and received acceleration data when the excitation frequency is $70 \mathrm{~Hz}$.

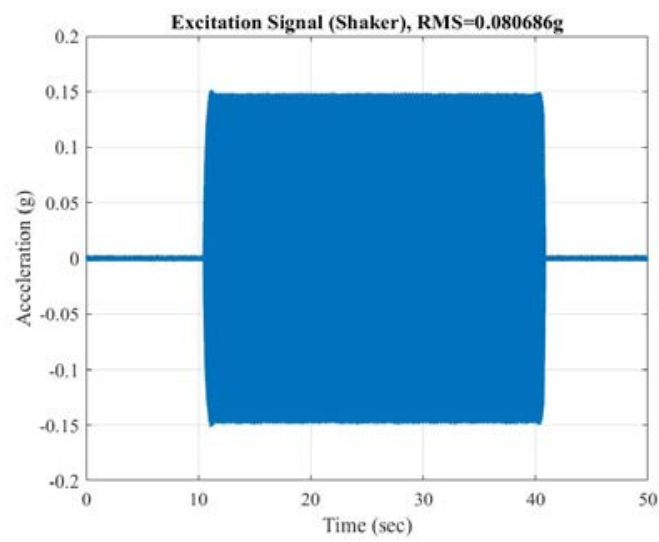

(a)

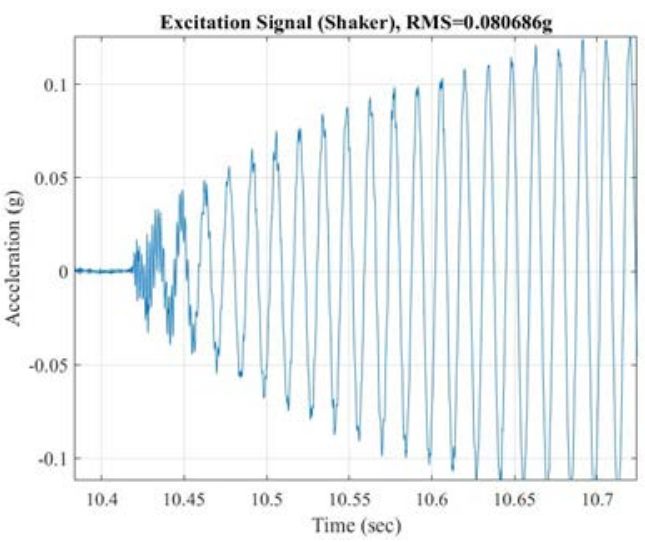

(b)

Figure 3.24: (a) Excitation Signal for Sinusoid of $70 \mathrm{~Hz}$ and (b) Its Closed View for Test Speed29 

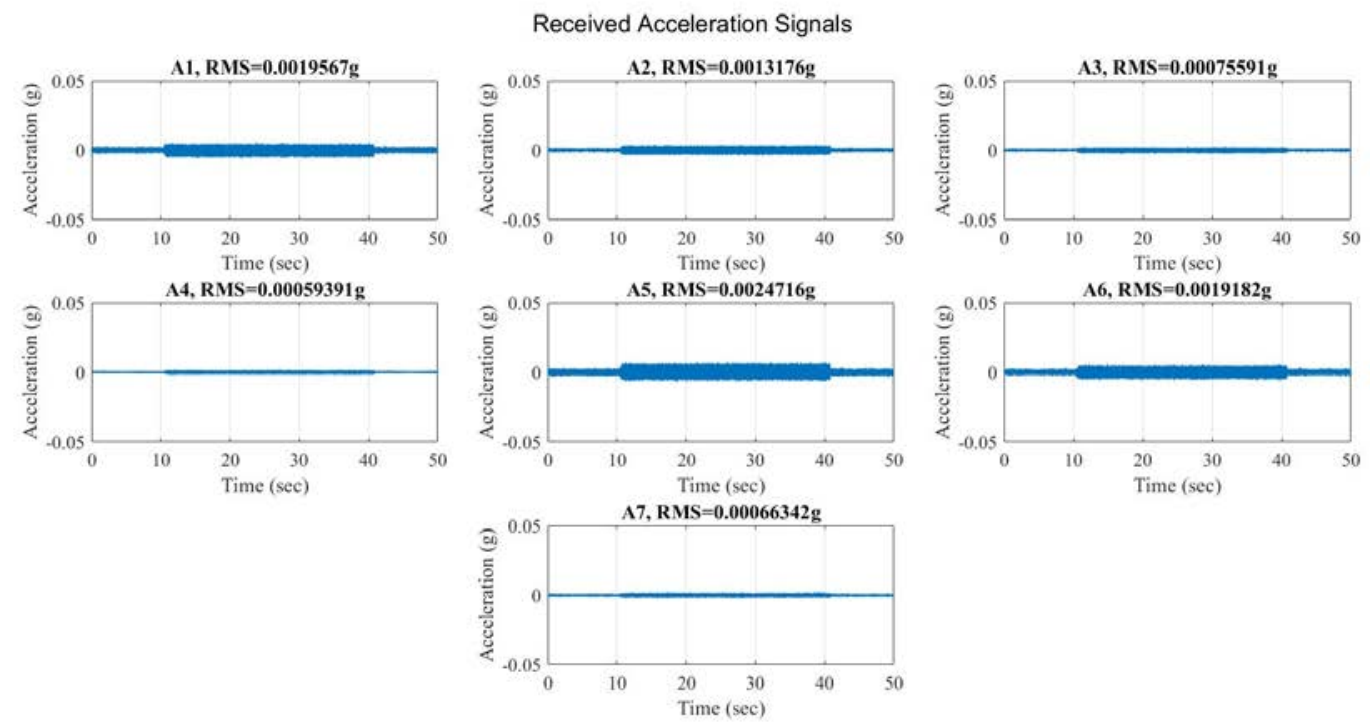

Figure 3.25: Received Signals due to Sinusoid Excitation with Frequency of $70 \mathrm{~Hz}$ for Test Speed 29

Similar to Section 3.5.1.1, the response accelerations for the A4 and A7 accelerometers are relatively weaker because they are farther away from the shaker and closer to the CMU wall and girder located on column lines E and 4, respectively. Figure 3.26 shows the PSD for both the excitation and the A2 accelerometer for the entire signal. Note that the Y-axis scale in these two plots is different. In this figure, using a Hamming window, the window length is 0.5 sec with a $75 \%$ overlap and a frequency resolution of 1 Hz. 


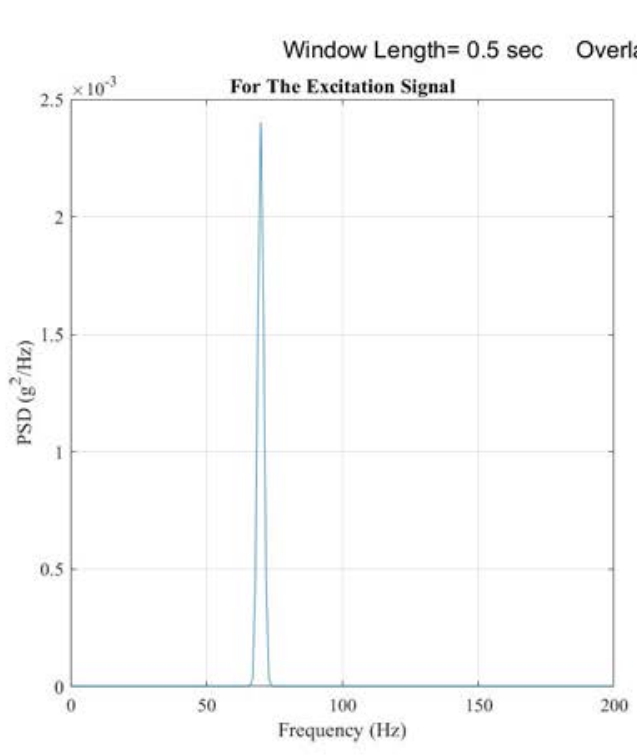

(a)

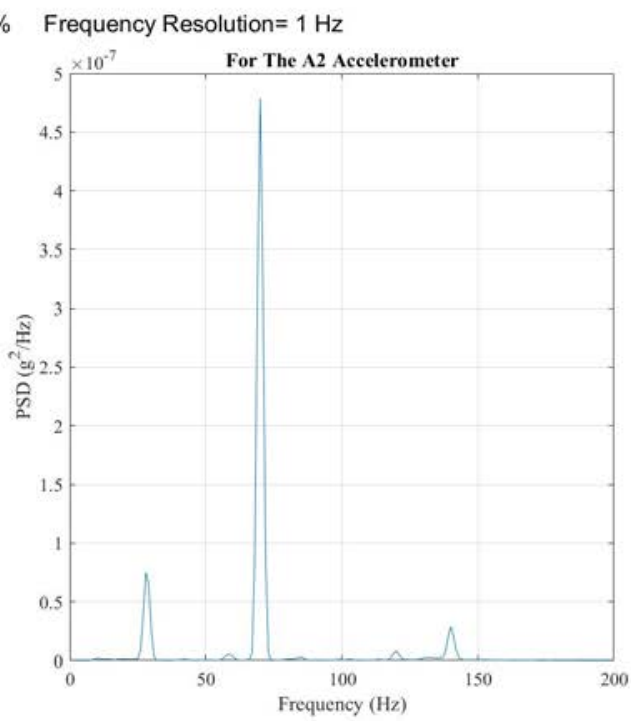

(b)

Figure 3.26: PSD for (a) Sinusoid Excitation with Frequency of $70 \mathrm{~Hz}$ and (b) A2 Accelerometer Response for Test Speed29

As expected, in Figure 3.26(a), there is a dominant peak with a frequency of $70 \mathrm{~Hz}$ for the excitation signal. The peak at $70 \mathrm{~Hz}$ in Figure 3.26(b) is also dominant, along with some other subordinate peaks at different frequencies.

Four different cases similar to Section 3.5.1.1 were considered here to investigate the influence of different processing techniques to reduce any possible noise. The four cases are listed below. Section 3.5.1.1. elucidated the details of and reasons for using each case. Moreover, the order of processing in each case as shown in Figure 3.17.

- Case 1: Entire signal with no manipulation

- $\quad$ Case 2: Entire signal + filtered in the frequency range of interest

- Case 3: Signal gated in time of interest + filtered in the frequency range of interest

- Case 4: Signal gated in time of interest + filtered in the frequency range of interest + partial whitening

\section{Case 1: Entire Signal with No Manipulation}

In this case, the cross-correlation for the entire signal between the excitation signal and each accelerometer was examined in the time-domain. In general, the cross-correlation 
between two periodic signals is also periodic. The overall shape of cross-correlation for two sinusoid signals is like a diamond, as shown in Figure 3.27. The similarity between the sinusoid excitation and its response signals is greater within the time delay between the two signals (i.e., top and bottom vertices of the diamond-shape below) and is less at the beginning and end (left and right vertices of the diamond-shape below).

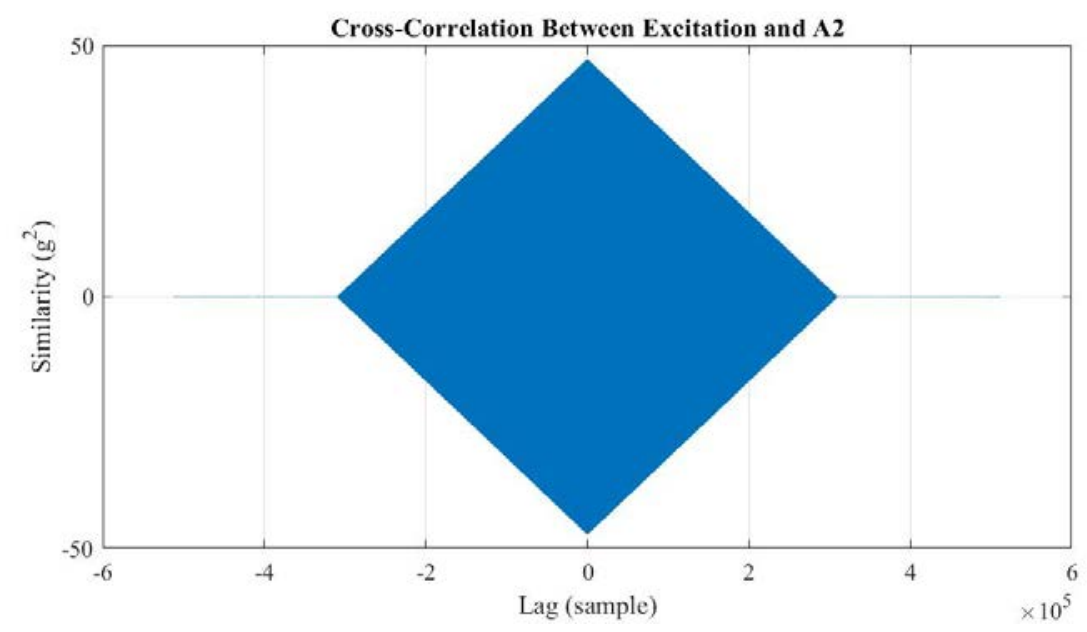

Figure 3.27: Cross-Correlation Function between Excitation and A2 Accelerometer for Test Speed29

Figure 3.28 shows the close-up of Figure 3.27 near zero. Unlike the crosscorrelation for the white noise, there is no single clear peak at the lag corresponding to the time delay. In Section 3.5.1.1, peaks in the cross-correlation due to the white noise excitation were ordered based on the highest similarity (see Figures 3.19 and 3.20 and see the order of peak numbers as an example) because peaks had different amplitudes close to the true time delay. However, in sinusoid excitation (Figure 3.28), the amplitudes of peaks in the cross-correlation are very close together; therefore, peak numbers will only be ordered for the positive peaks after zero lag from left to right. In Figure 3.28, the estimated time delays and corresponding propagation speeds are shown for the first five positive peaks. 


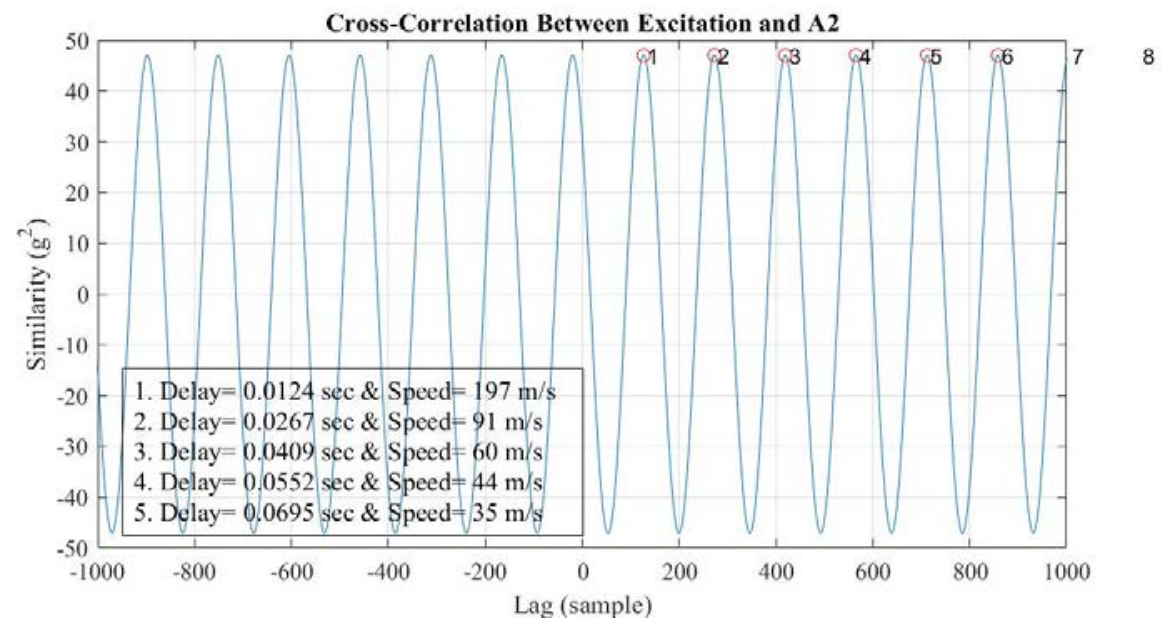

Figure 3.28: Close View of Cross-Correlation Function between Excitation and A2 Accelerometer for Test Speed 29

To determine which peak number corresponds to the true time delay, the first ten peaks were considered, as shown in Table 3.9. The estimated speeds shown in the text box in Figure 3.28 are represented in this table for the cross-correlation between the Arm and A2 accelerometers.

Table 3.9: Estimated Propagation Speed (Case 1: Entire Signal with No Manipulation) for Test Speed29

\begin{tabular}{|c|c|c|c|c|c|c|c|c|c|c|c|c|}
\hline \multirow{2}{*}{ Direction } & $\begin{array}{c}\text { Cross- } \\
\text { correlation }\end{array}$ & \multirow{2}{*}{ Distance } & \multicolumn{6}{|c|}{ Estimated Speed (m/s) for each Peak in Cross-Correlation } \\
\cline { 5 - 13 } & & 1st & 2nd & 3rd & 4th & 5th & 6th & 7th & 8th & 9th & 10th \\
\hline \multirow{3}{*}{$\mathrm{X}$} & Arm - A2 & 2.44 & 196.6 & 91.5 & 59.6 & 44.2 & 35.1 & 29.1 & 24.9 & 21.7 & 19.3 & 17.3 \\
\cline { 2 - 14 } & Arm - A3 & 4.88 & 648.6 & 223.9 & 135.3 & 96.8 & 75.4 & 61.8 & 52.3 & 45.4 & 40.0 & 35.8 \\
\cline { 2 - 14 } & Arm - A4 & 6.71 & 693.6 & 279.1 & 175.2 & 127.6 & 100.2 & 82.6 & 70.3 & 61.1 & 54.1 & 48.5 \\
\hline \multirow{3}{*}{ Y } & Arm - A5 & 1.22 & 222.9 & 61.8 & 35.9 & 25.2 & 19.5 & 15.9 & 13.4 & 11.6 & 10.2 & 9.1 \\
\cline { 2 - 13 } & Arm - A6 & 2.44 & 396.3 & 119.5 & 70.1 & 49.7 & 38.5 & 31.4 & 26.5 & 23.0 & 20.3 & 18.1 \\
\cline { 2 - 13 } & Arm - A7 & 3.66 & 407.1 & 157.4 & 97.5 & 70.7 & 55.3 & 45.5 & 38.7 & 33.6 & 29.7 & 26.6 \\
\hline
\end{tabular}

The estimated speed that is similar among the different accelerometers in each direction may be the true wave propagation speed in that direction. For example, the estimated speeds computed based on the cross-correlation between Arm-A2, Arm-A3, and Arm-A4 are $59.6 \mathrm{~m} / \mathrm{s}, 61.8 \mathrm{~m} / \mathrm{s}$, and $61.1 \mathrm{~m} / \mathrm{s}$, respectively as highlighted in yellow, and are close to each other in X-direction. The first similar number between different pairs of accelerometers is considered the speed propagation in each case in that direction. 


\section{Case 2: Entire Signal + Filtered in the Frequency Range of Interest}

The only difference in this case compared to Case 1 is that the signal is pre-filtered in the frequency range of interest. The excitation signal frequency was $70 \mathrm{~Hz}$ so that the frequency-domain of the signal is filtered in a way to keep the frequency contents between $60 \mathrm{~Hz}$ and $80 \mathrm{~Hz}$, using the Butterworth filter. The way that the signal is filtered and the effect of filtering in the time-domain are shown in Figure 3.29(a) and 3.29(b), respectively.

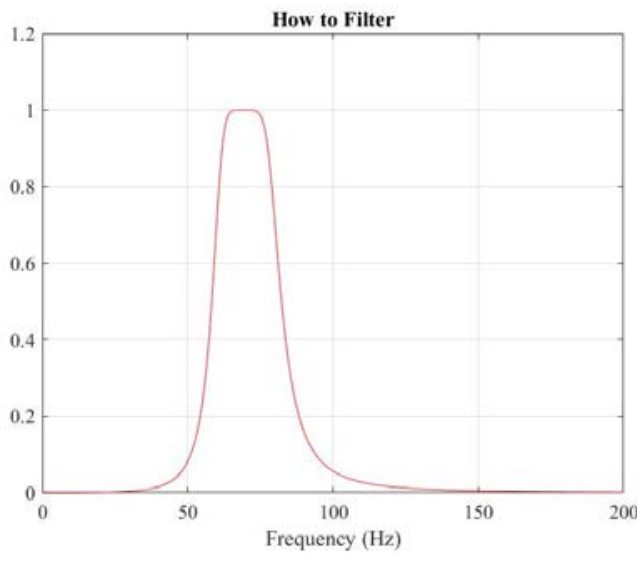

(a)

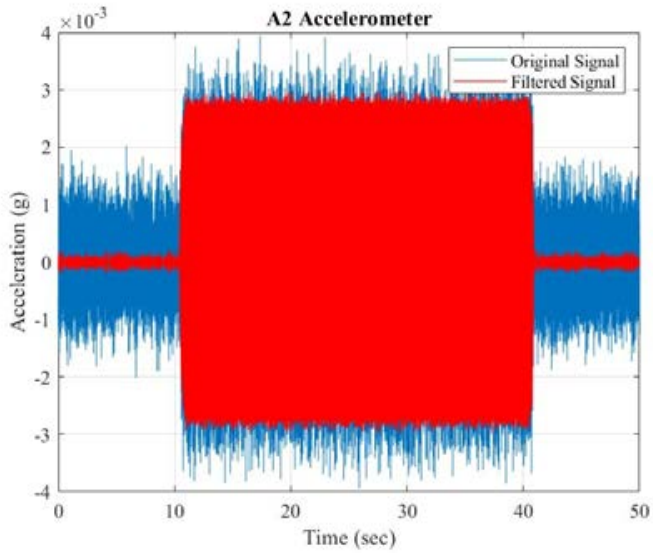

(b)

Figure 3.29: (a) The Way Signal Filtered in Frequency-Domain and (b) The Effect of Filtering in Time-Domain Signal of A2 Accelerometer for Test Speed29

As shown in Table 3.10, the results are almost identical to Case 1.

Table 3.10: Estimated Propagation Speed (Case 2: Entire Signal + Filtered in the Frequency Range of Interest) for Test Speed29

\begin{tabular}{|c|c|c|c|c|c|c|c|c|c|c|c|c|}
\hline \multirow{2}{*}{ Direction } & \multirow{2}{*}{$\begin{array}{c}\text { Cross- } \\
\text { correlation }\end{array}$} & \multirow{2}{*}{ Distance } & \multicolumn{10}{|c|}{ Estimated Speed $(\mathrm{m} / \mathrm{s})$ for each Peak in Cross-Correlation } \\
\hline & & & 1st & 2nd & 3rd & 4th & 5th & 6th & 7th & 8th & 9th & 10th \\
\hline \multirow{3}{*}{$\mathrm{X}$} & Arm - A2 & 2.44 & 198.2 & 91.5 & 59.6 & 44.2 & 35.1 & 29.1 & 24.9 & 21.7 & 19.3 & 17.3 \\
\hline & Arm - A3 & 4.88 & 648.6 & 223.9 & 135.3 & 96.8 & 75.4 & 61.8 & 52.3 & 45.4 & 40.0 & 35.8 \\
\hline & Arm - A4 & 6.71 & 693.6 & 279.1 & 175.2 & 127.6 & 100.2 & 82.6 & 70.3 & 61.1 & 54.1 & 48.5 \\
\hline \multirow{3}{*}{$\mathrm{Y}$} & Arm - A5 & 1.22 & 222.9 & 61.8 & 35.9 & 25.2 & 19.5 & 15.9 & 13.4 & 11.6 & 10.2 & 9.1 \\
\hline & Arm - A6 & 2.44 & 396.3 & 119.5 & 70.1 & 49.7 & 38.5 & 31.4 & 26.5 & 23.0 & 20.3 & 18.1 \\
\hline & Arm - A7 & 3.66 & 407.1 & 157.4 & 97.5 & 70.7 & 55.3 & 45.5 & 38.7 & 33.6 & 29.7 & 26.6 \\
\hline
\end{tabular}

\section{Case 3: Signal Gated in Time of Interest + Filtered in the Frequency Range of}

\section{Interest}

This case is similar to Case 2. The only difference is that a window length of two seconds of the signal was considered in the computation, which is shown in red in Figure 
3.30. The aim was to investigate if consideration of a smaller window would have any effect on the results. The two-second window length was chosen to consider the beginning of the signal immediately before the shaker started working, i.e., between $t=10$ sec to 12 sec. The justification for choosing this part of the signal is that the beginning of the signal (i.e., just before the shaker started working) may be corrupted less by noise.

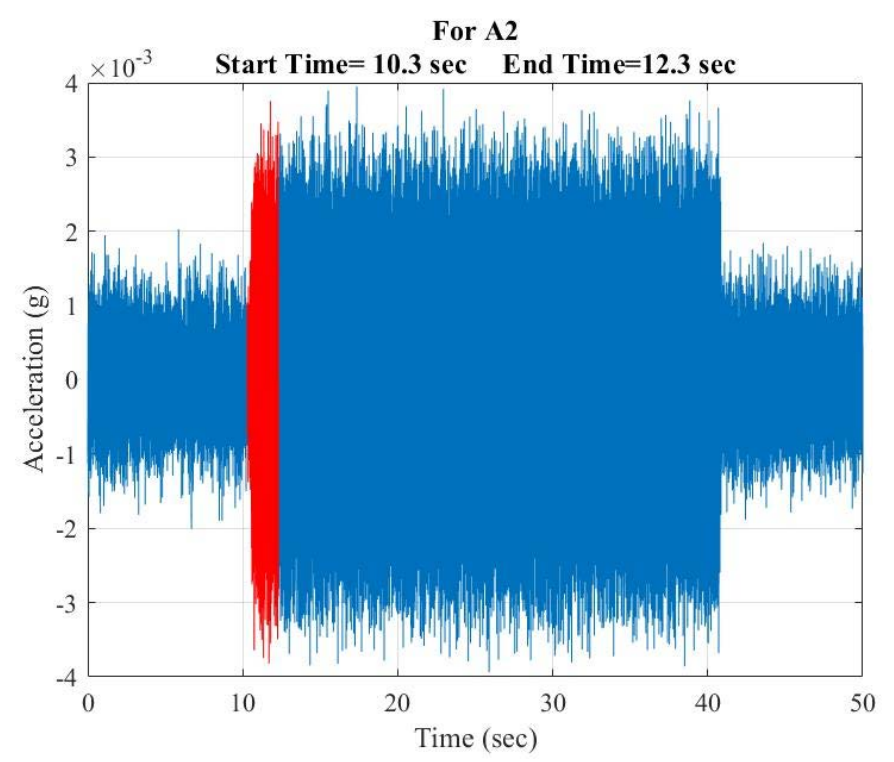

Figure 3.30: Signal Gated in Time of Interest for A2 Accelerometer for Test Speed29

The results for this case are shown in Table 3.11 and are almost identical to the results of cases 1 and 2. Other parts of the signal between $10 \mathrm{sec}$ to $40 \mathrm{sec}$ were also investigated using different window lengths, and the difference in results was negligible.

Table 3.11: Estimated Propagation Speed (Case 3: Signal Gated in Time of Interest + Filtered in the Frequency Range of Interest) for Test Speed 29

\begin{tabular}{|c|c|c|c|c|c|c|c|c|c|c|c|c|}
\hline \multirow{2}{*}{ Direction } & \multirow{2}{*}{$\begin{array}{c}\text { Cross- } \\
\text { correlation }\end{array}$} & \multirow{2}{*}{ Distance } & \multicolumn{6}{|c|}{ Estimated Speed (m/s) for each Peak in Cross-Correlation } \\
\cline { 5 - 13 } & & & 1st & 2nd & 3rd & 4th & 5th & 6th & 7th & 8th & 9th & 10th \\
\hline \multirow{3}{*}{ X } & Arm - A2 & 2.44 & 198.2 & 91.5 & 59.6 & 44.2 & 35.1 & 29.1 & 24.9 & 21.7 & 19.3 & 17.3 \\
\cline { 2 - 13 } & Arm - A3 & 4.88 & 648.6 & 223.9 & 135.3 & 96.8 & 75.4 & 61.8 & 52.3 & 45.4 & 40.0 & 35.8 \\
\cline { 2 - 13 } & Arm - A4 & 6.71 & 693.6 & 279.1 & 175.2 & 127.6 & 100.4 & 82.6 & 70.3 & 61.1 & 54.1 & 48.5 \\
\hline \multirow{3}{*}{ Y } & Arm - A5 & 1.22 & 222.9 & 61.8 & 35.9 & 25.2 & 19.5 & 15.9 & 13.4 & 11.6 & 10.2 & 9.1 \\
\cline { 2 - 12 } & Arm - A6 & 2.44 & 396.3 & 119.5 & 70.1 & 49.7 & 38.5 & 31.4 & 26.5 & 23.0 & 20.3 & 18.1 \\
\cline { 2 - 12 } & Arm - A7 & 3.66 & 407.1 & 157.4 & 97.5 & 70.7 & 55.3 & 45.5 & 38.7 & 33.6 & 29.7 & 26.6 \\
\hline
\end{tabular}

Case 4: Signal Gated in Time of Interest + Filtered in The Frequency Range of Interest + Partial Whitening 
The only difference between this case and the previous one is the addition of a postprocessing technique called partial whitening, which is used to reduce the effect of any possibly existing reflection in the signal. The effect of $20 \%$ partial whitening on both the time-domain and frequency-domain for the A2 accelerometer is shown in Figure 3.31.

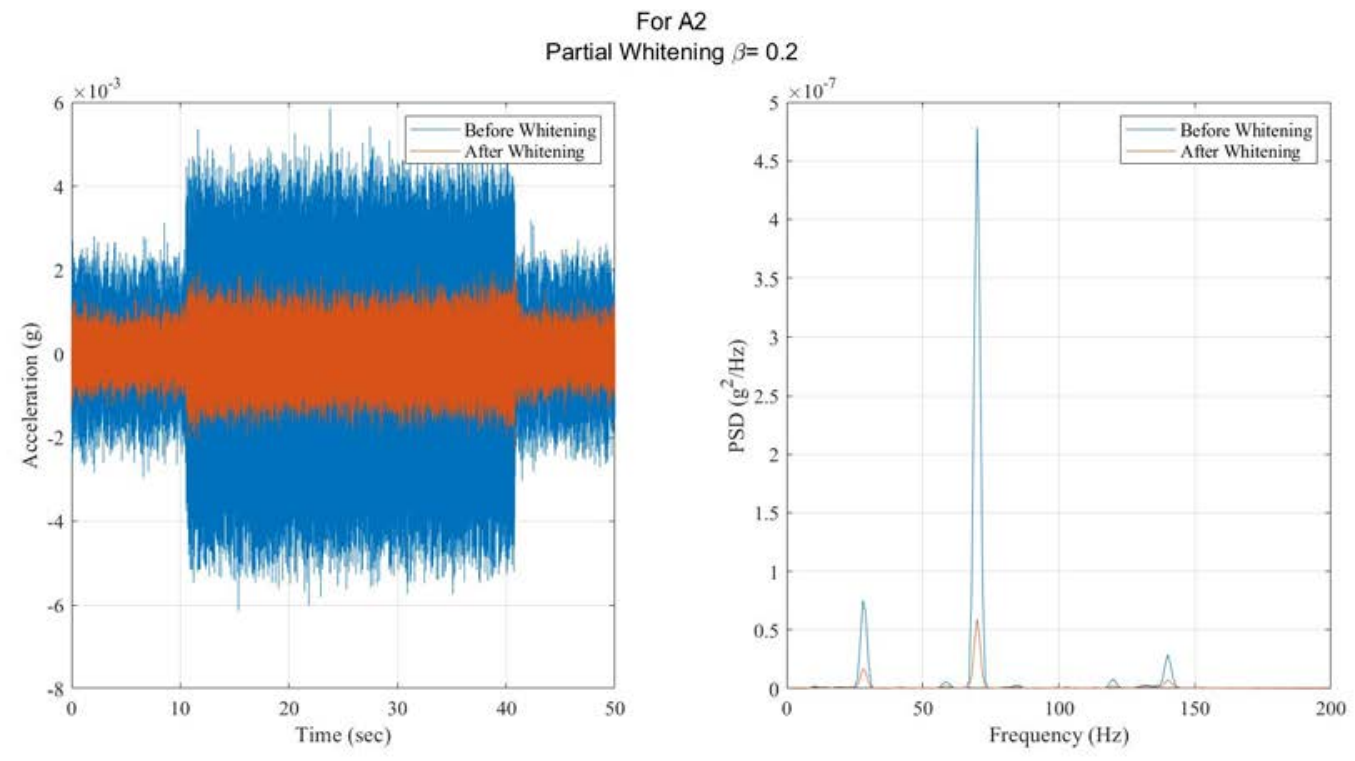

Figure 3.31: Effect of Partial Whitening on (a) Time-Domain and (b) Frequency-Domain for A2 Accelerometer for Test Speed29

Table 3.12 shows the results in this case, which are almost identical to the three previous cases. Different degrees of partial whitening have also been investigated, and the results for up to $70 \%$ partial whitening were almost the same.

Table 3.12: Estimated Propagation Speed (Case 4: Signal Gated in Time of Interest +

Filtered in The Frequency Range of Interest + Partial Whitening) for Test Speed29

\begin{tabular}{|c|c|c|c|c|c|c|c|c|c|c|c|c|}
\hline \multirow{2}{*}{ Direction } & \multirow{2}{*}{$\begin{array}{c}\text { Cross- } \\
\text { correlation }\end{array}$} & \multirow{2}{*}{ Distance } & \multicolumn{6}{|c|}{ Estimated Speed (m/s) for each Peak in Cross-Correlation } \\
\cline { 5 - 13 } & & & 1st & 2nd & 3rd & 4th & 5th & 6th & 7th & 8th & 9th & 10th \\
\hline \multirow{3}{*}{ X } & Arm - A2 & 2.44 & 198.2 & 91.5 & 59.6 & 44.2 & 35.1 & 29.1 & 24.9 & 21.7 & 19.3 & 17.3 \\
\cline { 2 - 13 } & Arm - A3 & 4.88 & 648.6 & 223.9 & 135.3 & 96.8 & 75.4 & 61.8 & 52.3 & 45.4 & 40.0 & 35.8 \\
\cline { 2 - 13 } & Arm - A4 & 6.71 & 693.6 & 279.1 & 175.2 & 127.6 & 100.2 & 82.6 & 70.3 & 61.1 & 54.1 & 48.5 \\
\hline \multirow{3}{*}{ Y } & Arm - A5 & 1.22 & 222.9 & 61.8 & 35.8 & 25.2 & 19.5 & 15.9 & 13.4 & 11.6 & 10.2 & 9.1 \\
\cline { 2 - 12 } & Arm - A6 & 2.44 & 396.3 & 119.5 & 70.1 & 49.7 & 38.5 & 31.4 & 26.5 & 23.0 & 20.2 & 18.1 \\
\cline { 2 - 12 } & Arm - A7 & 3.66 & 411.6 & 157.4 & 97.5 & 70.7 & 55.4 & 45.5 & 38.7 & 33.6 & 29.7 & 26.6 \\
\hline
\end{tabular}

Results shown in Tables 3.9 to 3.12 were based on the test Speed29. For comparison, Table 3.13 shows the results for both tests Speed29 and Speed30 when the 
shaker power was set to $50 \%$ and $75 \%$, respectively. There is almost no difference in the estimated WPS between these two tests.

Table 3.13: Speed Propagation Estimation for Speed29 and Speed30 Due to $70 \mathrm{~Hz}$ Sinusoid Excitation

\begin{tabular}{|c|c|c|c|c|c|c|c|c|c|}
\hline & & \\
\hline & \multirow{3}{*}{ Pair } & \multicolumn{8}{|c|}{ Estimated Speed (m/s) } \\
\hline & & \multicolumn{4}{|c|}{ Speed29 (Power Shaker 50\%) } & \multicolumn{4}{|c|}{ Speed30 (Power Shaker 75\%) } \\
\hline & & Case 1 & Case 2 & Case 3 & Case 4 & Case 1 & Case 2 & Case 3 & Case 4 \\
\hline \multirow{3}{*}{ X-Dir } & Arm - A2 & 59.6 & 59.6 & 59.6 & 59.6 & 59.5 & 59.5 & 59.5 & 59.5 \\
\hline & Arm - A3 & 61.8 & 61.8 & 61.8 & 61.8 & 61.7 & 61.7 & 61.7 & 61.7 \\
\hline & Arm - A4 & 61.1 & 61.1 & 61.1 & 61.1 & 61.0 & 61.0 & 61.1 & 61.1 \\
\hline \multirow{3}{*}{ Y-Dir } & Arm - A5 & 35.9 & 35.9 & 35.9 & 35.8 & 35.8 & 35.8 & 35.8 & 35.8 \\
\hline & Arm - A6 & 38.5 & 38.5 & 38.5 & 38.5 & 38.5 & 38.5 & 38.5 & 38.5 \\
\hline & Arm - A7 & 38.7 & 38.7 & 38.7 & 38.7 & 38.6 & 38.6 & 38.6 & 38.6 \\
\hline
\end{tabular}

Tables 3.14 and 3.15 show the estimated speed results due to $90 \mathrm{~Hz}$ and $110 \mathrm{~Hz}$ sinusoid excitation, respectively. As expected, the higher the frequency, the higher the estimated speed. This increase in estimated speed is higher in the shorter direction (i.e., Ydirection) than the longer direction (X-direction).

Table 3.14: Speed Propagation Estimation for Speed31 and Speed32 Due to $90 \mathrm{~Hz}$ Sinusoid Excitation

\begin{tabular}{|c|c|c|c|c|c|c|c|c|c|}
\hline \multicolumn{10}{|c|}{ SIIIUSUIU ExcildiüII } \\
\hline & \multirow{3}{*}{ Pair } & \multicolumn{8}{|c|}{ Estimated Speed (m/s) } \\
\hline & & \multicolumn{4}{|c|}{ Speed31 (Power Shaker 37.5\%) } & \multicolumn{4}{|c|}{ Speed32 (Power Shaker 50\%) } \\
\hline & & Case 1 & Case 2 & Case 3 & Case 4 & Case 1 & Case 2 & Case 3 & Case 4 \\
\hline \multirow{3}{*}{ X-Dir } & Arm - A2 & 72.0 & 72.0 & 72.0 & 72.0 & 72.0 & 72.0 & 72.0 & 72.0 \\
\hline & Arm - A3 & 77.1 & 77.1 & 77.1 & 77.1 & 76.9 & 76.9 & 76.9 & 76.9 \\
\hline & Arm - A4 & 74.4 & 74.4 & 74.4 & 74.4 & 74.3 & 74.3 & 74.3 & 74.3 \\
\hline \multirow{3}{*}{ Y-Dir } & Arm - A5 & 44.9 & 44.9 & 44.9 & 44.9 & 44.7 & 44.7 & 44.7 & 44.7 \\
\hline & Arm - A6 & 48.3 & 48.3 & 48.3 & 48.3 & 48.2 & 48.2 & 48.3 & 48.3 \\
\hline & Arm - A7 & 48.8 & 48.8 & 48.8 & 48.8 & 48.8 & 48.8 & 48.8 & 48.8 \\
\hline
\end{tabular}


Table 3.15: Speed Propagation Estimation for Speed33 and Speed34 Due to $110 \mathrm{~Hz}$ Sinusoid Excitation

\begin{tabular}{|c|c|c|c|c|c|c|c|c|c|}
\hline & \multirow{3}{*}{ Pair } & \multicolumn{8}{|c|}{ Estimated Speed (m/s) } \\
\hline & & \multicolumn{4}{|c|}{ Speed33 (Power Shaker 37.5\%) } & \multicolumn{4}{|c|}{ Speed34 (Power Shaker 50\%) } \\
\hline & & Case 1 & Case 2 & Case 3 & Case 4 & Case 1 & Case 2 & Case 3 & Case 4 \\
\hline \multirow{3}{*}{ X-Dir } & Arm - A2 & 104.0 & 104.0 & 104.5 & 104.5 & 101.5 & 101.5 & 102.8 & 102.8 \\
\hline & Arm - A3 & 104.5 & 104.5 & 104.9 & 104.9 & 103.4 & 103.4 & 103.8 & 103.8 \\
\hline & Arm - A4 & 108.8 & 108.8 & 109.0 & 109.0 & 107.8 & 107.8 & 108.1 & 108.1 \\
\hline \multirow{3}{*}{ Y-Dir } & Arm - A5 & 49.9 & 49.9 & 50.1 & 50.1 & 48.8 & 48.8 & 49.2 & 49.3 \\
\hline & Arm - A6 & 46.1 & 46.1 & 46.2 & 46.2 & 45.6 & 45.6 & 45.8 & 45.8 \\
\hline & Arm - A7 & 48.0 & 48 & 48.1 & 48.1 & 47.7 & 47.7 & 47.8 & 47.8 \\
\hline
\end{tabular}

\subsubsection{Frequency-Domain Approach}

In this section, all signals were first transferred to the frequency-domain and, then, the cross-spectrum between the excitation signal and each accelerometer was computed using Equation 2.16. The methodology was explained in Section 2.3.3. The following two sections provide empirical details for the WPS estimation while the excitation signal is either white noise or sinusoid.

\subsubsection{WPS Due to White Noise}

As mentioned previously, the generated white noise excitation was within a frequency range of $5 \mathrm{~Hz}$ to $200 \mathrm{~Hz}$, so this section considers only the frequency contents in this range. Three different cases were considered to investigate the effect of any possible noise on the results. The details of each case will be subsequently discussed. Note that the location of the accelerometers and the shaker is similar to Section 3.5.1.

- Case 1: Entire signal in the frequency range from $5 \mathrm{~Hz}$ to $200 \mathrm{~Hz}$

- Case 2: Signal gated in time of interest in the frequency range from $5 \mathrm{~Hz}$ to $200 \mathrm{~Hz}$

- Case 3: Signal gated in time of interest in the frequency range from $80 \mathrm{~Hz}$ to 180 $\mathrm{Hz}$

\section{Case 1: Entire Signal in the Frequency Range of $5 \mathrm{~Hz}$ to $200 \mathrm{~Hz}$}


With the use of the Fourier transform, all signals were transformed into the frequency-domain, and the cross-spectrum was computed using Equation 2.16. The gradient of the phase and weighting average of local gradients were computed (using Equations 2.17 and 2.18, respectively) to estimate the time delay first and then the propagation speed. Figure 3.32 shows the magnitude and phase for the Speed25 test between the excitation signal and the A2 accelerometer. Regarding Equation 3.8, which was used to compute the frequency resolution and consider the entire 50-second duration of the test, in this case, the frequency resolution was $0.02 \mathrm{~Hz}$. The red line in the phase plot specifies frequencies of interest between $5 \mathrm{~Hz}$ to $200 \mathrm{~Hz}$ for the time delay estimation (designated by E-Delay in the figure) and, as a result, the computation of the WPS in this frequency range (designated by E-Speed in the figure).

Between Excitaiton and A2 Accelerometer

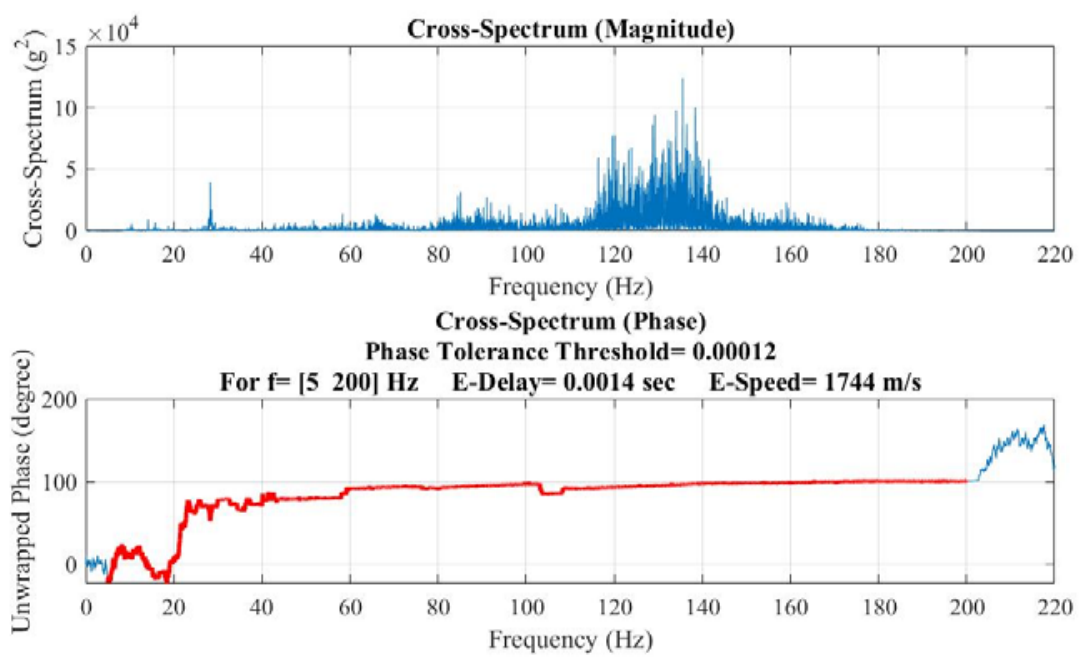

Figure 3.32: Cross-Spectrum between Excitation and A2 Accelerometer for Case 1

The cross-spectrum phase is the ratio of the imaginary part to the real part of the $\hat{P}(f)$ expressed by Equation 3.10. Minimal real-number values close to zero will amplify and manifest the incorrect phase results. Therefore, a threshold was defined to ignore all phase values below this threshold, which is called the Phase Tolerance Threshold, and it was defined as the maximum value of the cross-spectrum divided by $10^{9}$, which is 0.00012 , as shown in Figure 3.32. Note that the phase can be highly affected by an insufficiently small phase tolerance threshold, so it is essential to find the correct limit through a trial- 
and-error procedure and determine if the shape of the phase is almost the same in two or more consecutive trials. Table 3.16 shows the results for different phase tolerance thresholds and their corresponding estimated speeds. As can be seen in this table, the tolerance limit of $\frac{\max |\hat{A}|}{10^{6}}$ or smaller for the phase gives the same estimated speed. $\max |\hat{A}|$ is the maximum magnitude of the acceleration in the cross-spectrum.

$$
\operatorname{Phase}(f)=\tan ^{-1}\left(\frac{\hat{P}_{i m}(f)}{\hat{P}_{r e}(f)}\right)
$$

Table 3.16: Effect of Defined Phase Tolerance Threshold on Estimated Speed for A2 Accelerometer for Test Speed25

\begin{tabular}{|c|c|c|c|}
\hline $\begin{array}{c}\text { Cross- } \\
\text { Spectrum }\end{array}$ & $\begin{array}{c}\text { Defined Phase Tolerance } \\
\text { Threshold }\end{array}$ & $\begin{array}{c}\text { Phase Tolerance Threshold } \\
\text { in This Case }\end{array}$ & $\begin{array}{c}\text { Estimated Speed } \\
\mathbf{( m / s )}\end{array}$ \\
\hline \multirow{4}{*}{ Arm - A2 } & $\frac{\max |\hat{A}|}{10^{9}}$ & 0.00012 & 1744 \\
\cline { 2 - 4 } & $\frac{\max |\hat{A}|}{10^{8}}$ & 0.0012 & 1744 \\
\cline { 2 - 4 } & $\frac{\max |\hat{A}|}{10^{7}}$ & 0.012 & 1744 \\
\cline { 2 - 4 } & $\frac{\max |\hat{A}|}{10^{6}}$ & 0.12 & 1744 \\
\cline { 2 - 4 } & $\frac{\max |\hat{A}|}{10^{5}}$ & 1.2 & 1743 \\
\cline { 2 - 4 } & $\frac{\max |\hat{A}|}{10^{4}}$ & 12 & 1775 \\
\cline { 2 - 4 } & $\frac{\max |\hat{A}|}{10^{3}}$ & 120 & 2008 \\
\hline
\end{tabular}

The estimated time delay and its corresponding WPS for test Speed25 in the Xdirection for the A2 accelerometer is 0.0014 seconds and $1744 \mathrm{~m} / \mathrm{s}$, respectively. The results, in this case, are significantly higher than the estimated values in section 3.5.1.1. The results for all accelerometers for both white noise excitation tests, i.e., Speed 25 and Speed26, are shown in Table 3.18. The results of these two tests, which have different levels of shaker power, are significantly different.

\section{Case 2: Signal Gated in Time of Interest in the Frequency Range from 5 to 200}

$\mathrm{Hz}$

In this case, the processing is analogous to Case 1, except only a portion of the signal was considered. Figure 3.33 illustrates the time of interest shown in red for the test Speed25 for A2 accelerometer from $10.3 \mathrm{sec}$ to $12.3 \mathrm{sec}$, which was a two-second duration 
immediately before the shaker was operating. It should be noted that the Y-axis scale in these two figures is different.

Window starts from $10.3 \mathrm{sec}$ to $12.3 \mathrm{sec}$
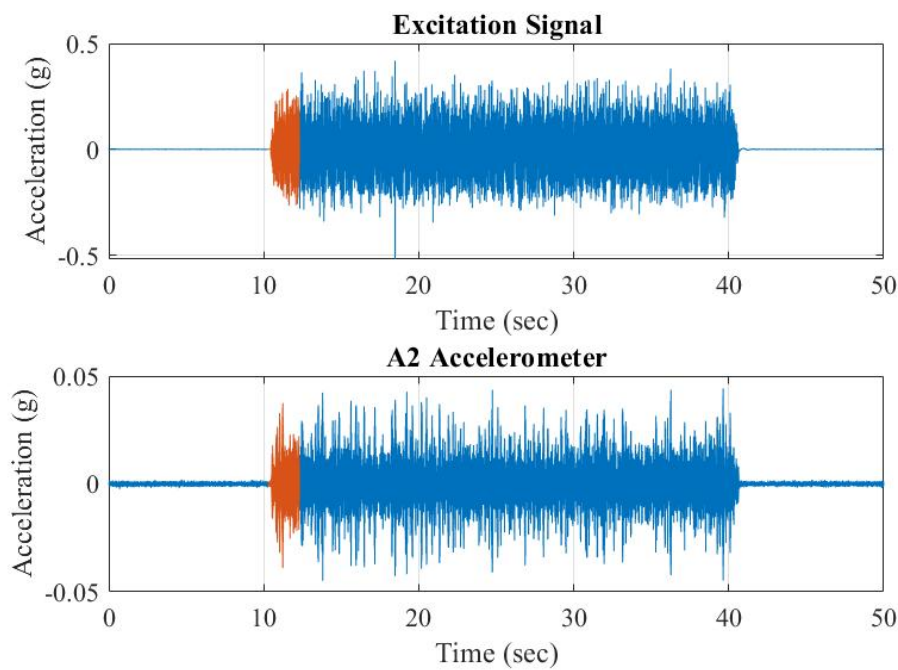

Figure 3.33: Time of Interest for Excitation and A2 Accelerometer for Case 2

Figure 3.34 shows the magnitude and phase of the cross-spectrum between the excitation and A2 accelerometer for the considered time of interest.

Between Excitaiton and A2 Accelerometer

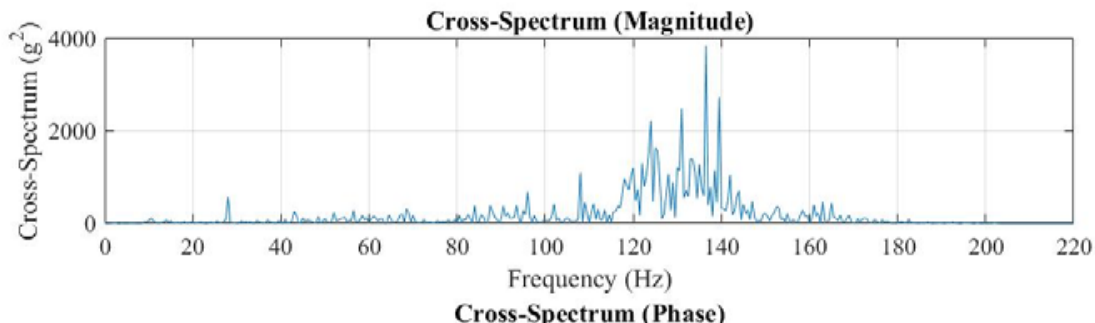

Phase Tolerance Threshold $=3.8 \mathrm{e}-06$

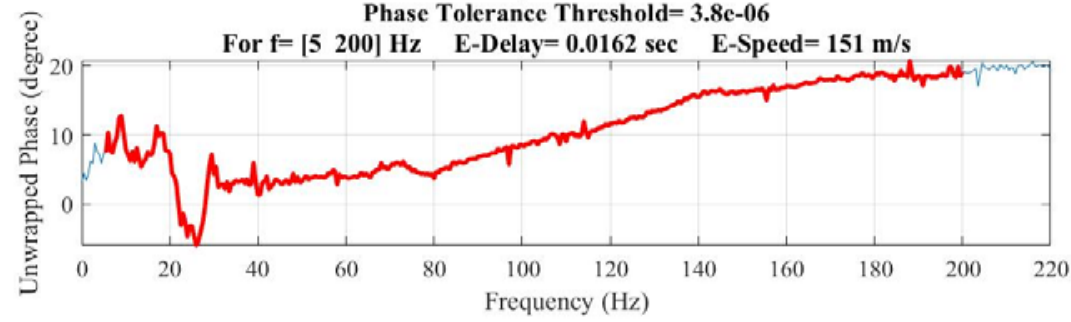

Figure 3.34: Cross-Spectrum between Excitation and A2 Accelerometer for Case 2

The results, in this case, are not reliable because the computation of the WPS was highly affected by both the time duration and selection of the time of interest in the time- 
domain. As an example, Table 3.17 shows the different time durations (fourth column) for the A2 accelerometer in different parts of the signal (second and third columns), with the last column designating the estimated speed of wave propagation. To further demonstrate that this time delay estimator is unreliable, Figure 3.35 shows another case when the WPS was computed as a negative number for test Speed26 between the excitation and A5 accelerometer.

Table 3.17: Effect of Selected Time Duration on Estimated Speed for A2 Accelerometer for Test Speed25

\begin{tabular}{|c|c|c|c|c|}
\hline $\begin{array}{c}\text { Cross- } \\
\text { Spectrum }\end{array}$ & $\begin{array}{c}\text { Start Time } \\
\text { (sec) }\end{array}$ & $\begin{array}{c}\text { End Time } \\
\text { (sec) }\end{array}$ & $\begin{array}{c}\text { Duration } \\
\text { (sec) }\end{array}$ & $\begin{array}{c}\text { Estimated } \\
\text { Speed (m/s) }\end{array}$ \\
\hline & 10.3 & 12.3 & 2 & 151 \\
\cline { 2 - 5 } & 10.3 & 13.3 & 3 & 798.3 \\
\cline { 2 - 5 } & 10.3 & 15.3 & 5 & 583.8 \\
\cline { 2 - 5 } & 10.3 & 30.3 & 20 & 4285 \\
\cline { 2 - 5 } & 10.3 & 40.3 & 30 & 1005 \\
\cline { 2 - 5 } & 20 & 22 & 2 & 187.3 \\
\cline { 2 - 5 } & 15 & 30 & 15 & 1191 \\
\hline
\end{tabular}

Between Excitaiton and A5 Accelerometer
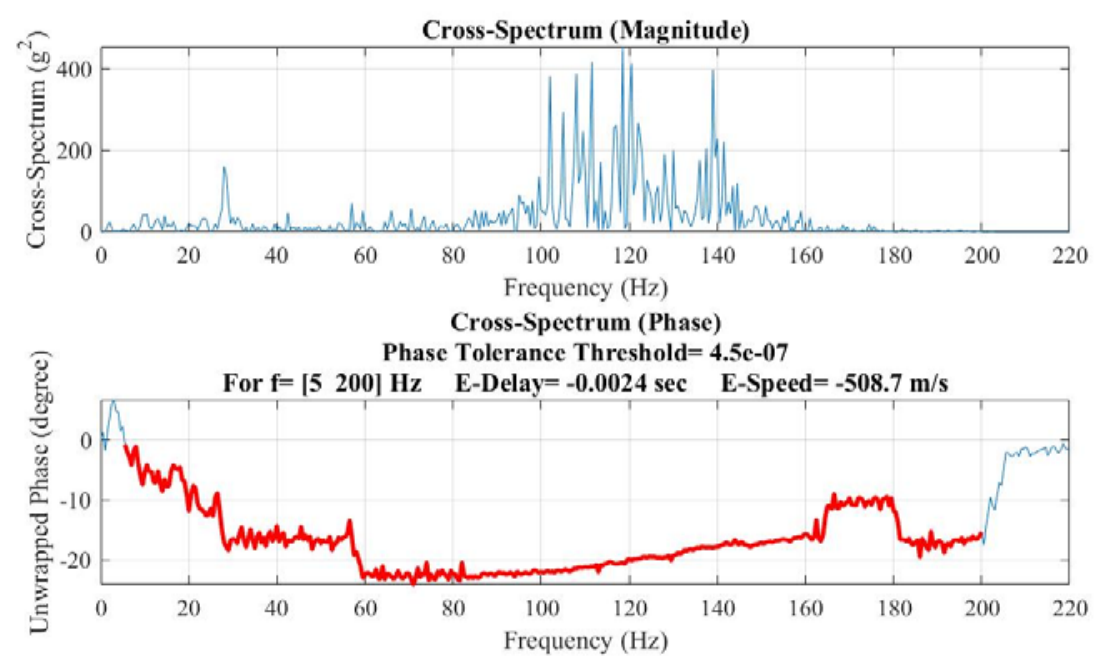

Figure 3.35: Negative Estimated Speed for Test Speed26 and for A5 Accelerometer for Time of Interest from 11-13 sec 
Table 3.18 shows the results for all the accelerometers in this case using a twosecond time duration window length immediately before the shaker was operating (see Figure 3.33).

\section{Case 3: Signal Gated in Time of Interest in the Frequency Range of $80 \mathrm{~Hz}$ to}

\section{$180 \mathrm{~Hz}$}

This section aims to investigate the effect of a smaller frequency band to estimate the time delay. Therefore, a range from $80 \mathrm{~Hz}$ to $180 \mathrm{~Hz}$ for the phase was chosen because the coherence of this range was more than $80 \%$. Figure 3.36 shows an example of this frequency range, as shown in red, for the A2 accelerometer for test Speed25 within a time of interest from $10.3 \mathrm{sec}$ to $12.3 \mathrm{sec}$.

Between Excitaiton and A2 Accelerometer

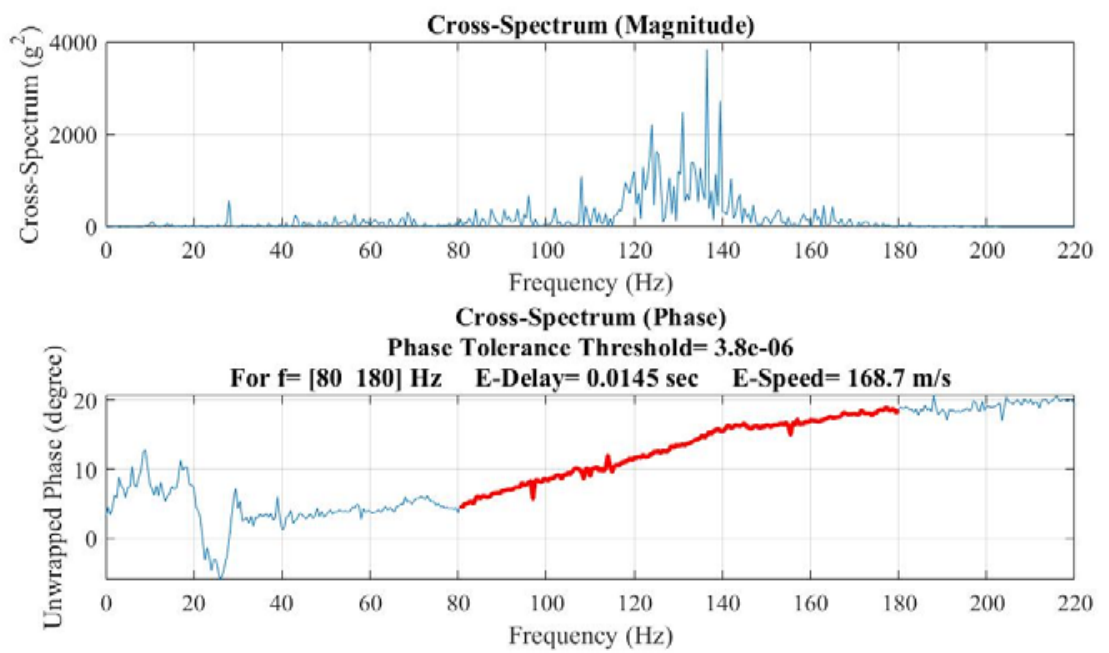

Figure 3.36: Cross-Spectrum between Excitation and A2 Accelerometer for the Frequency Range from $80-180 \mathrm{~Hz}$ for Case 3, for Test Speed25 and Time of Interest From 10.3-12.3 sec

The results for all the pairs of accelerometers for all three cases are shown in Table 3.18. 
Table 3.18: Estimated Speed for Frequency Approach Due to White Noise Excitation

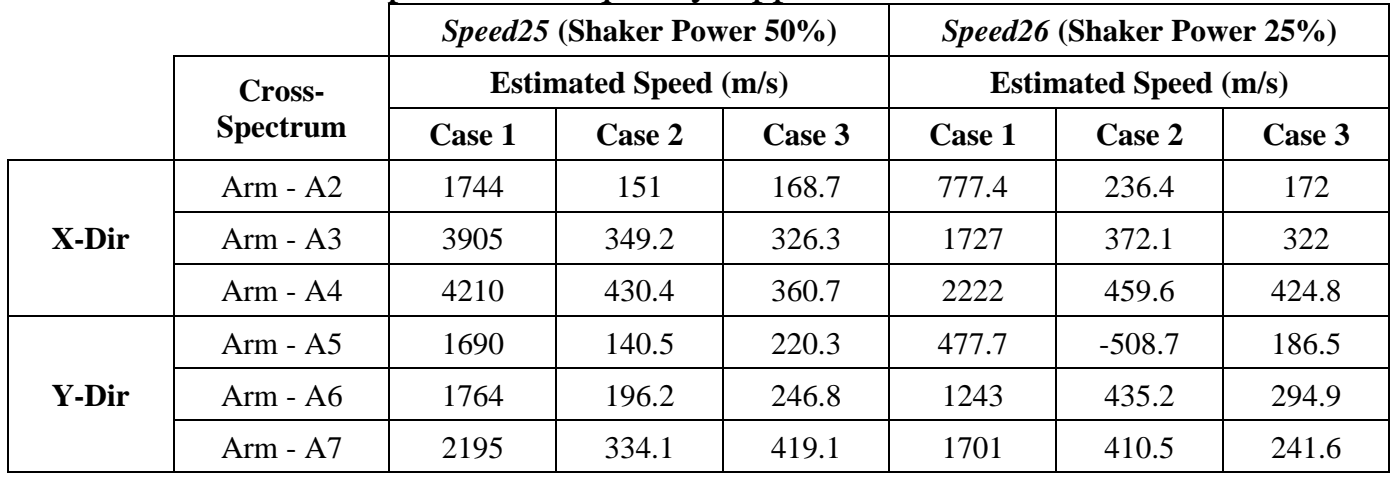

The frequency approach did not work well to estimate the time delay, which is a prerequisite step for computing the wave propagation speed. There is no way to verify the estimated speeds between different pairs of accelerometers using the frequency approach. Trying three different cases did not produce any promising results. Therefore, the results shown in Table 3.18 will not be used in Section 3.6. However, although the results of the frequency approach were not promising, all details were presented here for future research and improvement.

\subsubsection{WPS Due to Sinusoid}

As mentioned previously, there were some tests on the sinusoid excitation with frequencies of $70 \mathrm{~Hz}, 90 \mathrm{~Hz}$, and $110 \mathrm{~Hz}$ (single sinusoid on each test), as shown in Table 3.8. In this section, the frequency approach was employed to estimate the time delay and then the propagation speed. Two different cases were considered to investigate the effect of different post-processing techniques on the results:

- Case 1: Entire signal in the frequency range of interest

- Case 2: Signal gated in time of interest and the frequency range of interest.

\section{Case 1: Entire Signal in Frequency Range of Interest}

In this case, the entire time-domain signal was used in the post-processing. For the test on sinusoid excitation with a frequency of $70 \mathrm{~Hz}$, different ranges of frequencies with $70 \mathrm{~Hz}$ at the middle of these ranges - were needed to compute the time delay. This was a trial-and-error procedure since there is no guidance on how wide this frequency 
range should be for such an application. Table 3.19 shows the results for the different frequency ranges for the test at $70 \mathrm{~Hz}$ sinusoid excitation when the entire time-domain signal is considered.

Table 3.19: Estimated Speed for the Entire Signal in Different Frequency Ranges due to $70 \mathrm{~Hz}$ Sinusoid Excitation

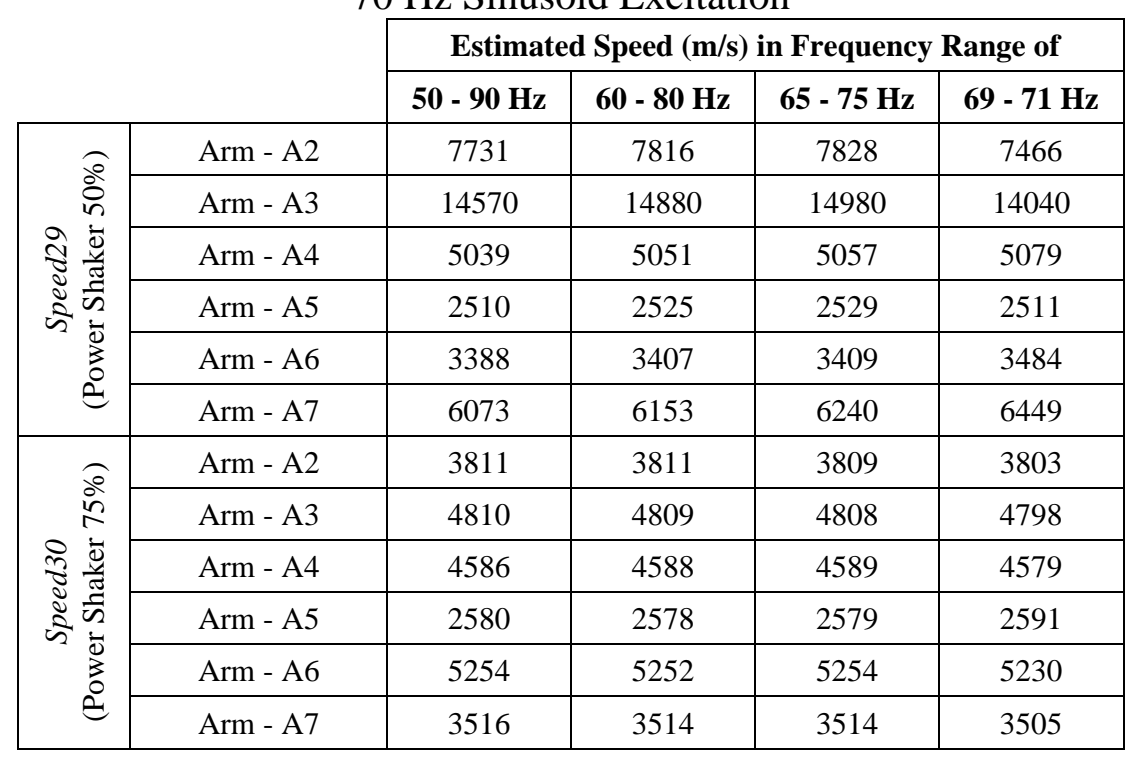

As shown above, the results are significantly higher than Section 3.5.1.2. However, the variation in the estimated speed in each case for the sinusoid excitation is smaller compared to the white noise excitation results (Table 3.18).

The same procedures are also applied for other tests with sinusoid excitations of 90 $\mathrm{Hz}$ and $110 \mathrm{~Hz}$ frequencies. Tables 3.20 and 3.21 show the results for these two tests. Similar to Table 3.19, each table contains two tests with two different shaker power levels. It was evident that the results are not reliable since there are huge differences among the estimated WPS for the different pairs of accelerometers. It can be seen in Table 3.21 that the estimated time delay is a negative value in some cases, resulting in a negative estimated speed. 
Table 3.20: Estimated Speed for the Entire Signal in Different Frequency Ranges due to $90 \mathrm{~Hz}$ Sinusoid Excitation

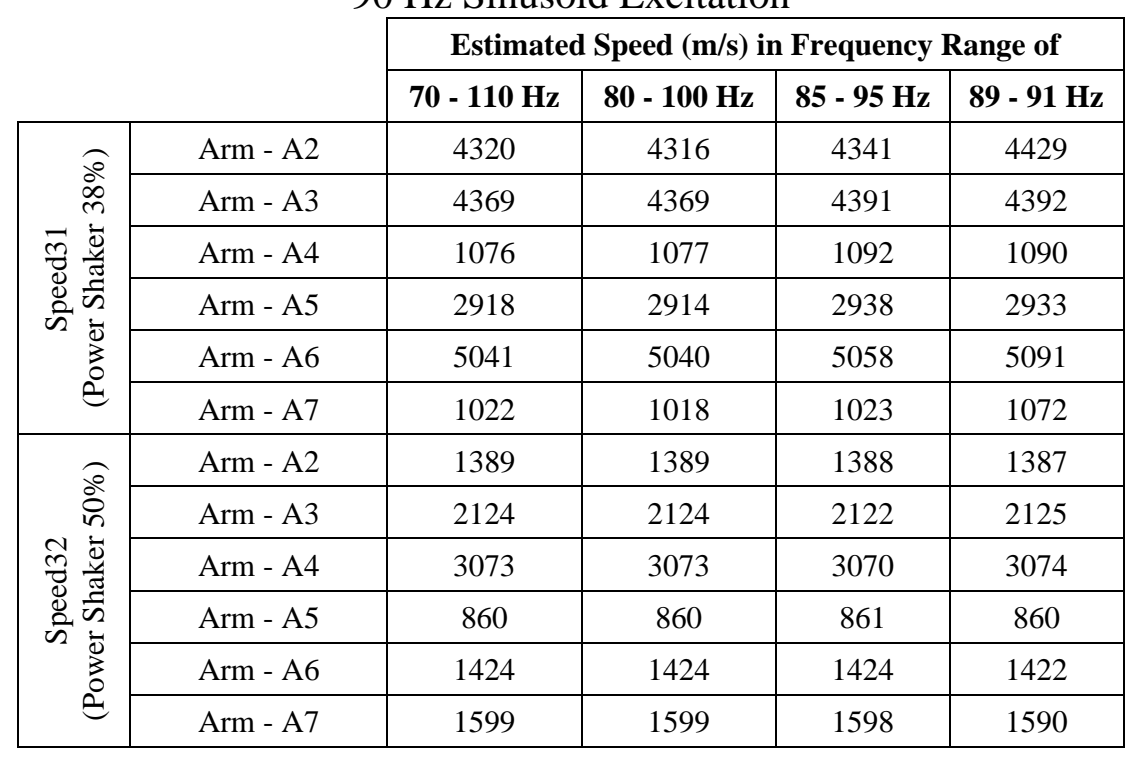

Table 3.21: Estimated Speed for the Entire Signal in Different Frequency Ranges due to $110 \mathrm{~Hz}$ Sinusoid Excitation

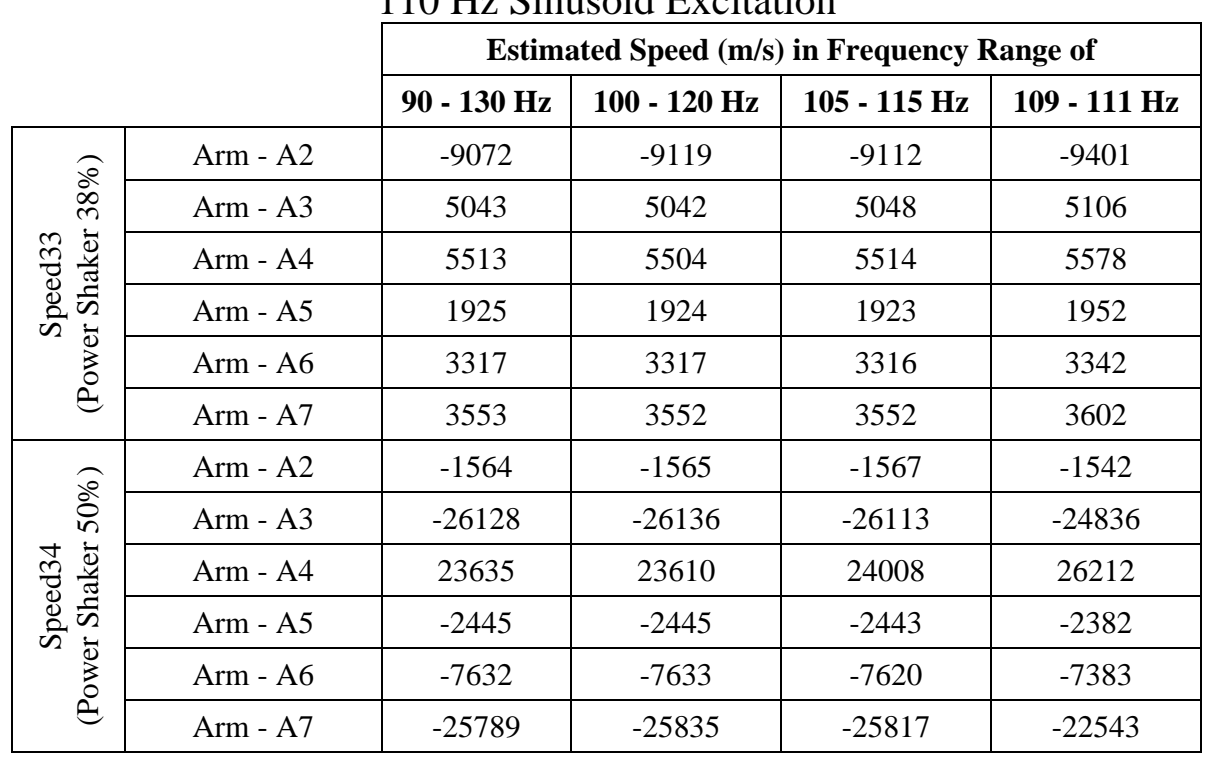

\section{Case 2: Signal Gated in Time of Interest and Frequency Range of Interest}

The only difference in this case compared to Case 1 is that only a segment of the signal was considered when estimating the propagation speed. Table 3.22 shows the results of different start and end times for the purpose of WPS estimation. Due to the lack of correlation among the estimated speeds as well as the negative values, the frequency 
approach seems to be unreliable for this application. Therefore, no table is added in this case for the $90 \mathrm{~Hz}$ and $110 \mathrm{~Hz}$ tests.

Table 3.22: Effect of Selected Time Duration on Estimated Speed for A2 Accelerometer for Test Speed29

\begin{tabular}{|c|c|c|c|c|}
\hline \multirow{4}{*}{ Cross-Spectrum } & $\begin{array}{c}\text { Start Time } \\
\text { (sec) }\end{array}$ & $\begin{array}{c}\text { End Time } \\
\text { (sec) }\end{array}$ & $\begin{array}{c}\text { Duration } \\
\text { (sec) }\end{array}$ & $\begin{array}{c}\text { Estimated } \\
\text { Speed (m/s) }\end{array}$ \\
\hline \multirow{7}{*}{ Arm - A2 } & 11 & 13 & 2 & -267 \\
\cline { 2 - 5 } & 12 & 14 & 2 & 85 \\
\cline { 2 - 5 } & 20 & 22 & 2 & 52 \\
\cline { 2 - 5 } & 11 & 15 & 4 & -159 \\
\cline { 2 - 5 } & 20 & 25 & 5 & -495 \\
\cline { 2 - 5 } & 25 & 35 & 10 & -529 \\
\hline
\end{tabular}

\subsubsection{Summary}

Section 3.5 aimed to estimate the time delay as a prerequisite step for computing the wave propagation speed or WPS. More precisely, this section attempted to determine the time delay estimation by examining two different approaches using cross-correlation in the time-domain and the cross-spectrum in the frequency-domain. In both approaches, different cases have been defined for two purposes. First, to minimize the inclusion of any possible noise in the acquired signals and make the method more robust against the possible noise. Second, to investigate the influence of different post-processing techniques in the computation of WPS. The results were compared between different pairs of accelerometers to assess and determine the validity of the estimated values in each approach. This study shows the superior performance of the time-domain implementation in processing acceleration data acquired from a concrete floor. Variations in the estimated time delays in the time-domain approach are fairly small in each case. However, the frequency-domain approach's lack of robustness is shown by the fact that the variation among the different cases was considerably high. Indeed, in some cases, the time delay was estimated to be a negative number. The results of the time-domain approach will be used in the next section, locating the source of vibration. 
Although the frequency approach did not yield promising results, this may be applicable in other applications or circumstances. Nevertheless, this data is included for completeness.

\subsection{Localization}

The ranges of WPS for the white noise and sinusoid excitation with different frequencies have been computed in Section 3.5.1 in both X- and Y-directions, separately. These ranges will be employed to localize a vibration source using the SRP method. All the tests in this section were conducted in a single bay between column lines 3-4 and F-E, as shown in Figure 3.5. This area is also shown in Figure 3.37. The blue lines represent the walls. Moreover, the origin coordinate of all tests in Section 3.6 is shown in Figure 3.37. Note that the origin and direction of the $\mathrm{X}$ and $\mathrm{Y}$ axes in this section (Figure 3.37) are different from those shown in Section 3.5 (Figure 3.7). Four locations were chosen for the shaker as the source of vibration and are denoted by L1 to L4 in Figure 3.37, which shows the coordinates of each source location. The first and second numbers in the parentheses represent the $\mathrm{X}$ and $\mathrm{Y}$ coordinates with respect to the origin shown by red in Figure 3.37. The accelerometers were distributed randomly in the tested area, and their coordinates are shown in Table 3.24.

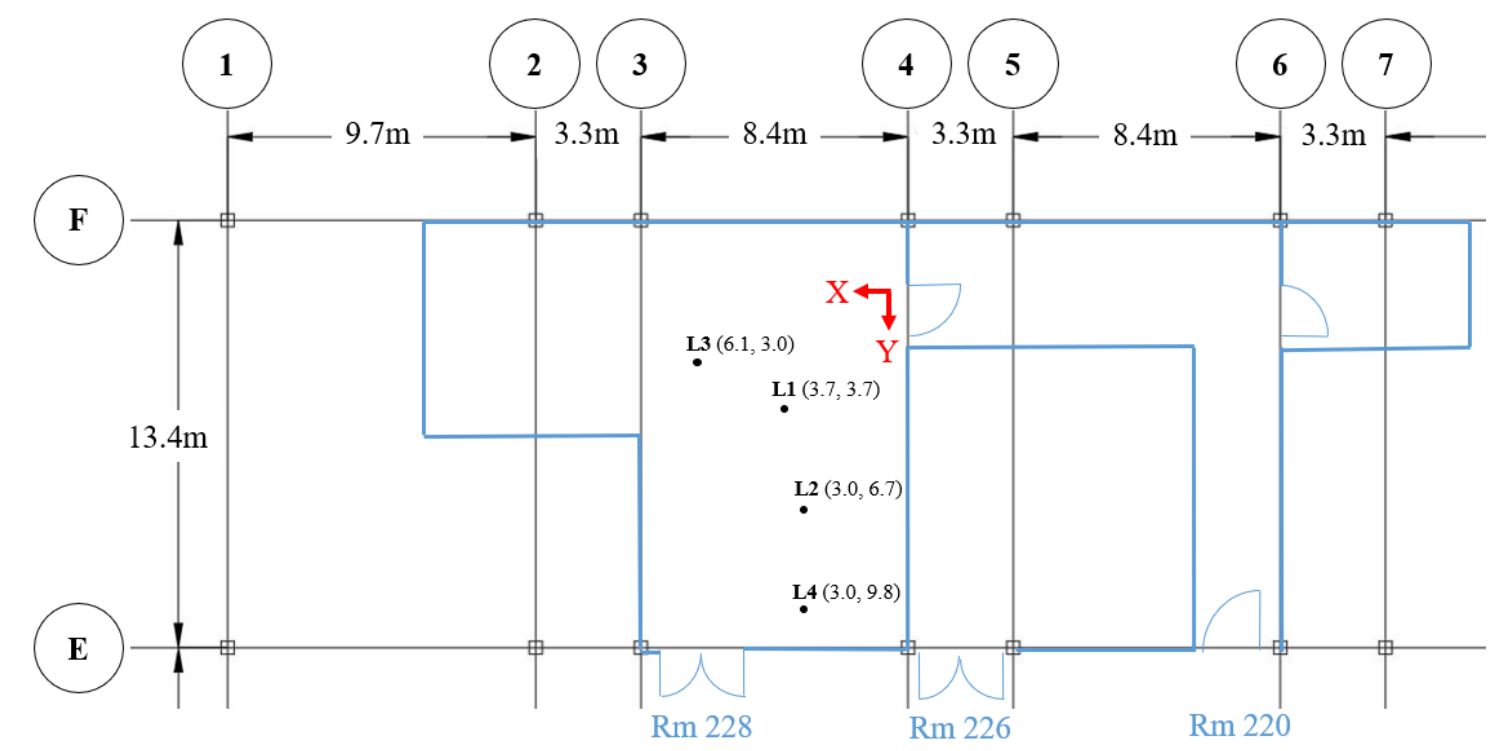

Figure 3.37: Tested Area Between Column Lines 3-4 and F-E 


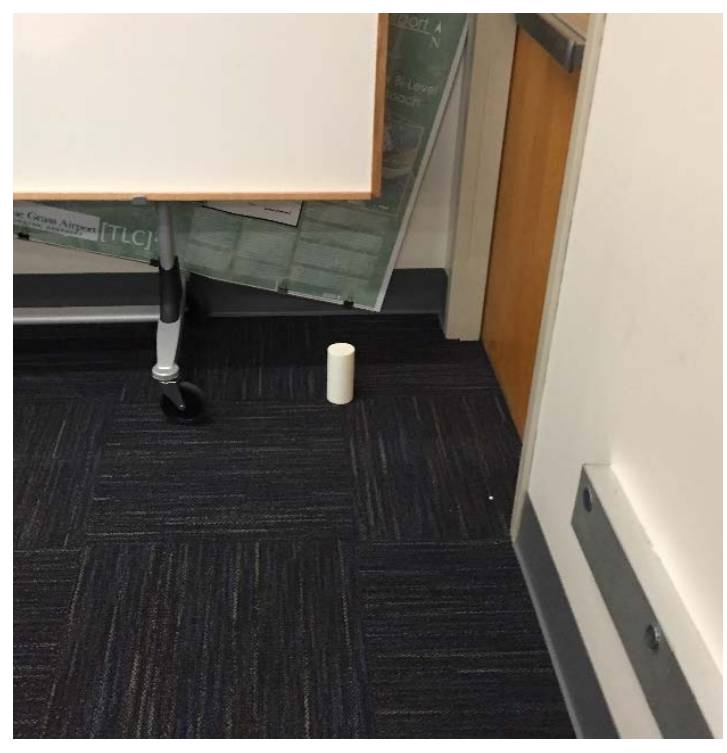

(a) The Origin in The Tested Floor

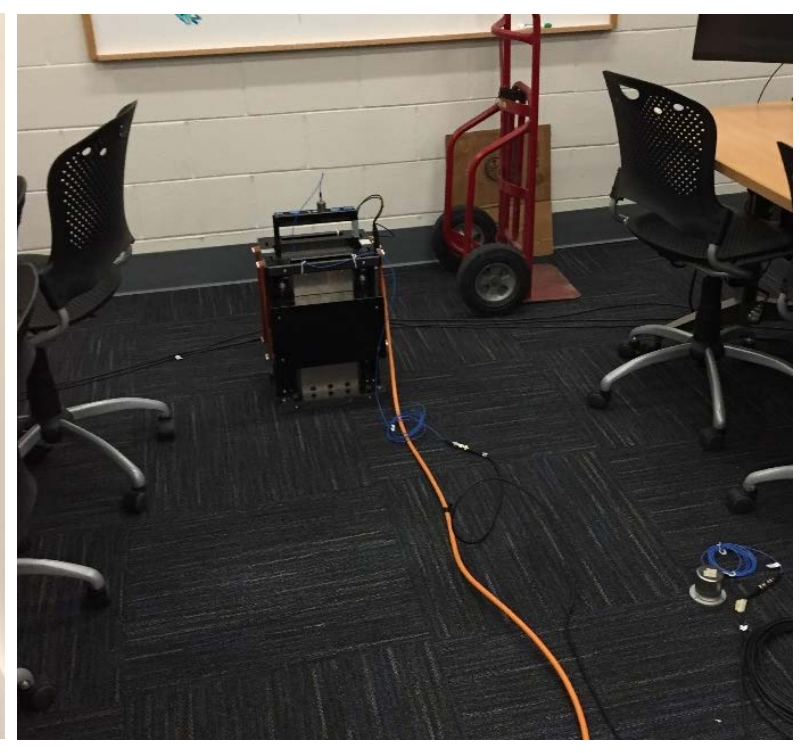

(b) Shaker at Location L4

Figure 3.38: Photos of The Origin and The Shaker in The Tested Floor

Table 3.23: Shaker Locations

\begin{tabular}{|c|c|c|}
\hline $\begin{array}{c}\text { Shaker } \\
\text { Location }\end{array}$ & $\begin{array}{c}\text { X Coordinate } \\
(\mathbf{m})\end{array}$ & $\begin{array}{c}\text { Y Coordinate } \\
\text { (m) }\end{array}$ \\
\hline L1 & 3.7 & 3.7 \\
\hline L2 & 3.05 & 6.71 \\
\hline L3 & 6.10 & 3.05 \\
\hline L4 & 3.05 & 9.75 \\
\hline
\end{tabular}

Table 3.24: Accelerometer Locations

\begin{tabular}{|c|c|c|}
\hline $\begin{array}{c}\text { Shaker } \\
\text { Location }\end{array}$ & $\begin{array}{c}\text { X Coordinate } \\
(\mathbf{m})\end{array}$ & $\begin{array}{c}\text { Y Coordinate } \\
\text { (m) }\end{array}$ \\
\hline $\mathrm{A} 1$ & 4.27 & 1.22 \\
\hline $\mathrm{A} 2$ & 5.49 & 4.88 \\
\hline $\mathrm{A} 3$ & 3.66 & 8.53 \\
\hline $\mathrm{A} 4$ & 6.10 & 9.75 \\
\hline $\mathrm{A} 5$ & 1.22 & 7.32 \\
\hline $\mathrm{A} 6$ & 2.44 & 3.66 \\
\hline $\mathrm{A} 7$ & 1.22 & 0.61 \\
\hline
\end{tabular}

Since the propagation speed for each excitation force is different in $\mathrm{X}$ - and $\mathrm{Y}$ directions, the WPS can be computed from Equation 3.11: 


$$
W P S=\frac{d_{x}}{d_{x}+d_{y}} V_{x}+\frac{d_{y}}{d_{x}+d_{y}} V_{y}
$$

where $d_{x}$ and $d_{y}$ are the distance in $\mathrm{X}$ - and Y-directions between each candidate point and assumed source location in each iteration. $V_{x}$ and $V_{y}$ are the estimated WPS in $\mathrm{X}$ - and Y-directions in Section 3.5.1.

\subsubsection{Localization of White Noise Excitation}

As mentioned in Section 3.5.1.1, Table 3.7, the WPS in the X- and Y-directions caused by the white noise excitation yielded $72-80 \mathrm{~m} / \mathrm{s}$ and 102-113 m/s, respectively. Note that the WPS mentioned above is valid only for the tested bay when the excitation signal is white noise with a frequency range of $5 \mathrm{~Hz}$ to $200 \mathrm{~Hz}$. The SRP method uses an estimated WPS to locate the vibration source. Each vibration test was repeated twice with different shaker power at each location. The tests in this section are listed in Table 3.25.

Table 3.25: List of White Noise Excitation Tests

\begin{tabular}{|c|c|c|c|c|}
\hline Test Name & $\begin{array}{c}\text { Shaker } \\
\text { Location }\end{array}$ & Excitation & $\begin{array}{c}\text { Frequency } \\
\text { Range } \\
\text { Hz }\end{array}$ & $\begin{array}{c}\text { Shaker } \\
\text { Power }\end{array}$ \\
\hline OHR2nd-L1-1 & L1 & White Noise & $5-200$ & $50 \%$ \\
\hline OHR2nd-L1-2 & L1 & White Noise & $5-200$ & $25 \%$ \\
\hline OHR2nd-L2-1 & L2 & White Noise & $5-200$ & $50 \%$ \\
\hline OHR2nd-L2-2 & L2 & White Noise & $5-200$ & $25 \%$ \\
\hline OHR2nd-L3-1 & L3 & White Noise & $5-200$ & $50 \%$ \\
\hline OHR2nd-L3-2 & L3 & White Noise & $5-200$ & $25 \%$ \\
\hline OHR2nd-L4-1 & L4 & White Noise & $5-200$ & $50 \%$ \\
\hline OHR2nd-L4-2 & L4 & White Noise & $5-200$ & $25 \%$ \\
\hline
\end{tabular}

The configuration of the tests OHR2nd-L1-1 and OHR2nd-L1-2 is shown in Figure 3.39 . 


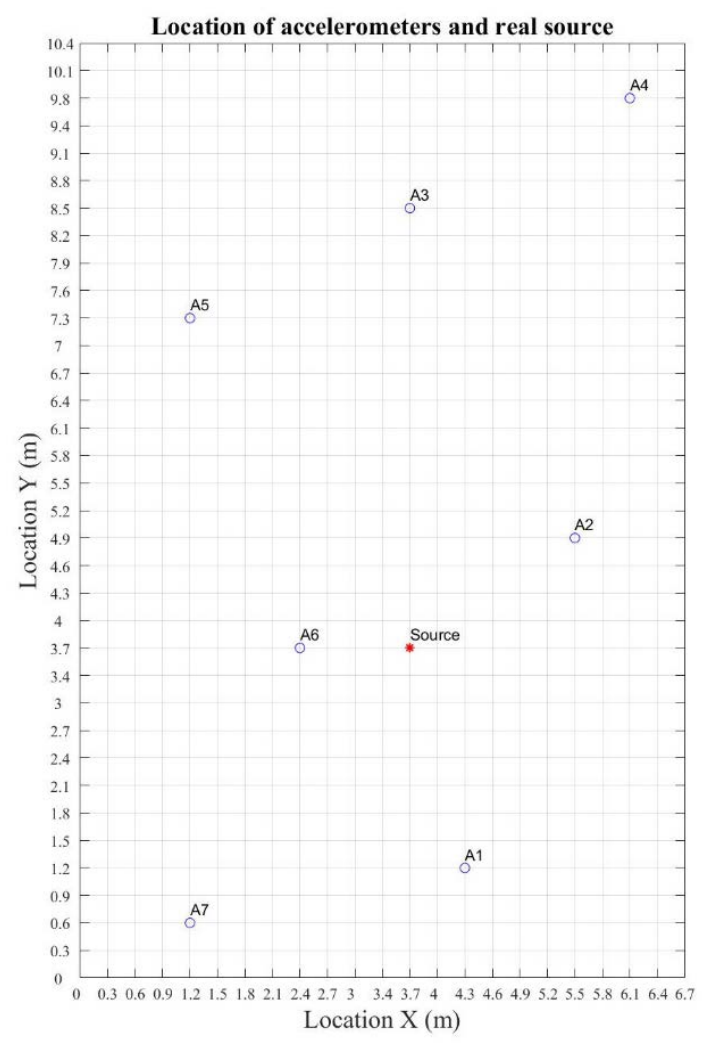

Figure 3.39: Test Setup for Tests When the Shaker was Placed at L1

Figure 3.40 shows the time-domain data for test OHR2nd-L1-1. Interestingly, despite being farthest from the source, the A4 accelerometer did not report the weakest received acceleration. Indeed, the weakest received acceleration data was reported by the A5 accelerometer. However, the A6 and A2 accelerometers were closer to the shaker than the others and received the strongest vibration signals. Figure 3.40 also includes the RMS value to compare the vibration levels received by each accelerometer. 


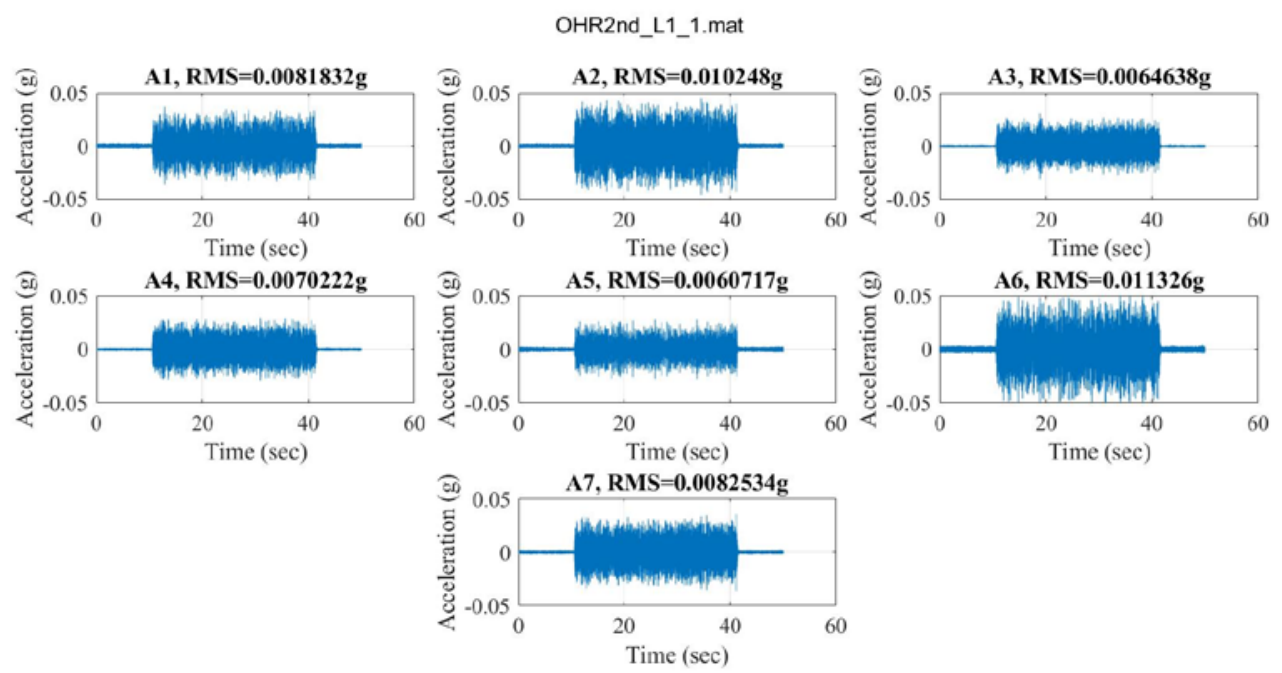

Figure 3.40: Time-Domain Data for Test OHR2nd-L1-1

As explained in Chapter 2, with the use of inverse distance weighting (Equation 2.12), less weight can be given to the accelerometers farther away from the selected candidate point to compensate for the effect of attenuation, which helps to significantly reduce the ambiguity of the SRP plot. Figure 3.41 shows the SRP results based on different weight distribution scenarios. Figure 3.41(a) shows the result when all the accelerometers make an equal contribution, which is not a correct assumption in the presence of attenuation. Figures 3.41(b) to 3.41(e) show different levels of decaying powers $-b$ in Equation 2.12 - and corresponding steered powers. The real and estimated vibration source locations are also shown in this figure with plus and circle marks, respectively.

Note that in the plots like the ones shown in Figure 3.41, the name of the test for which the SRP plot was generated is stated at the top. The SRP plot can only be generated for specific wave propagation speeds in the $\mathrm{X}$ - and $\mathrm{Y}$-directions, which are also stated at the top of each SRP plot. Error represents the localization error, which measures the distance between the real and estimated sources. In addition, the coordinates of the estimated and real sources are included. 


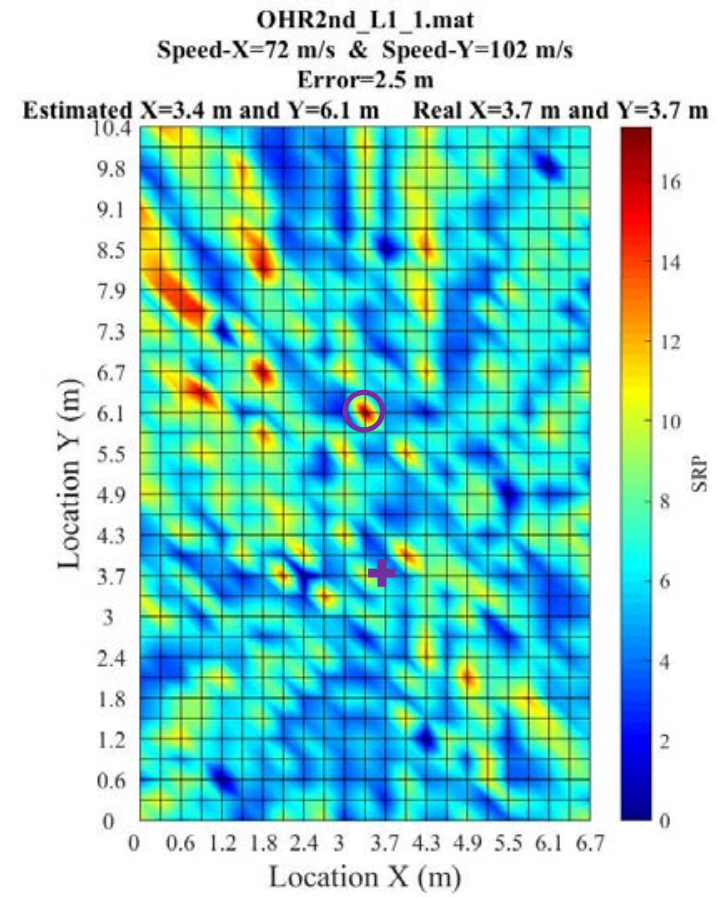

(a) $b=0$, Equal Contribution

OHR2nd_L1_1.mat

Speed-X=72 m/s \& Speed-Y=102 m/s

Error $=1.5 \mathrm{~m}$

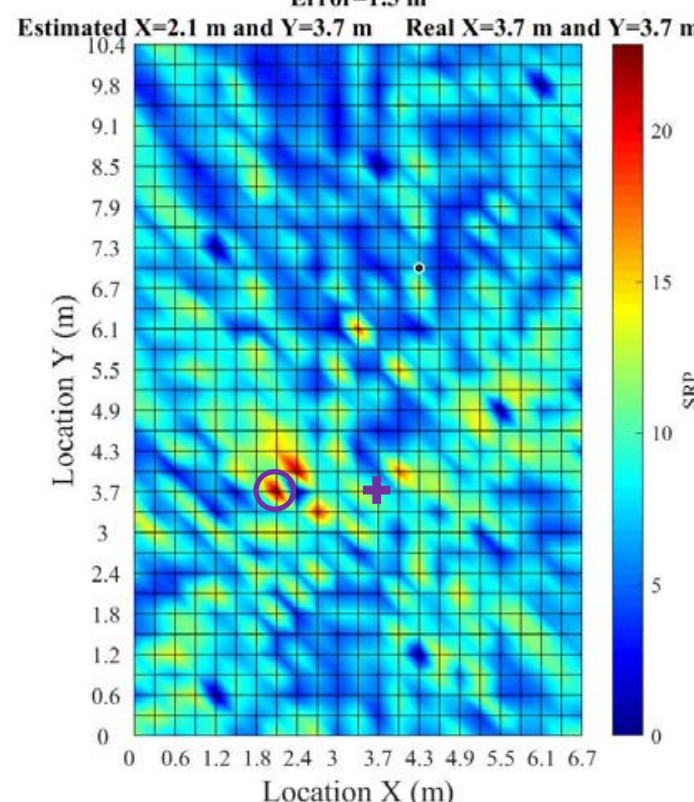

Location X (m)
OHR2nd_L1_1.mat
Speed-X $=72 \mathrm{~m} / \mathrm{s} \&$ Speed-Y=102 m/s

Error $=2.5 \mathrm{~m}$

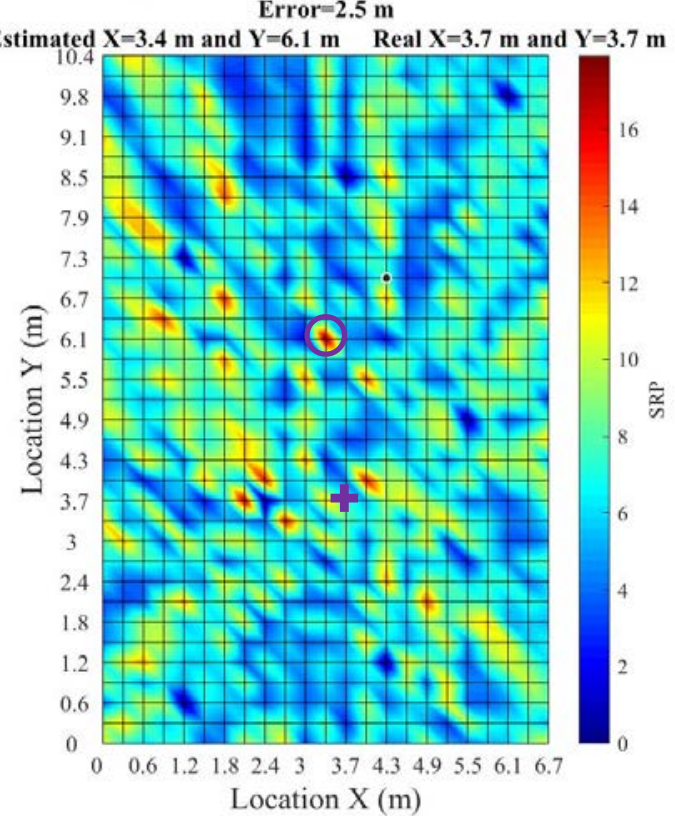

(b) $b=0.25$

\author{
OHR2nd_L1_1.mat \\ 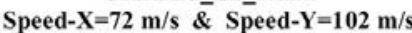 \\ Error=1.5 m
}

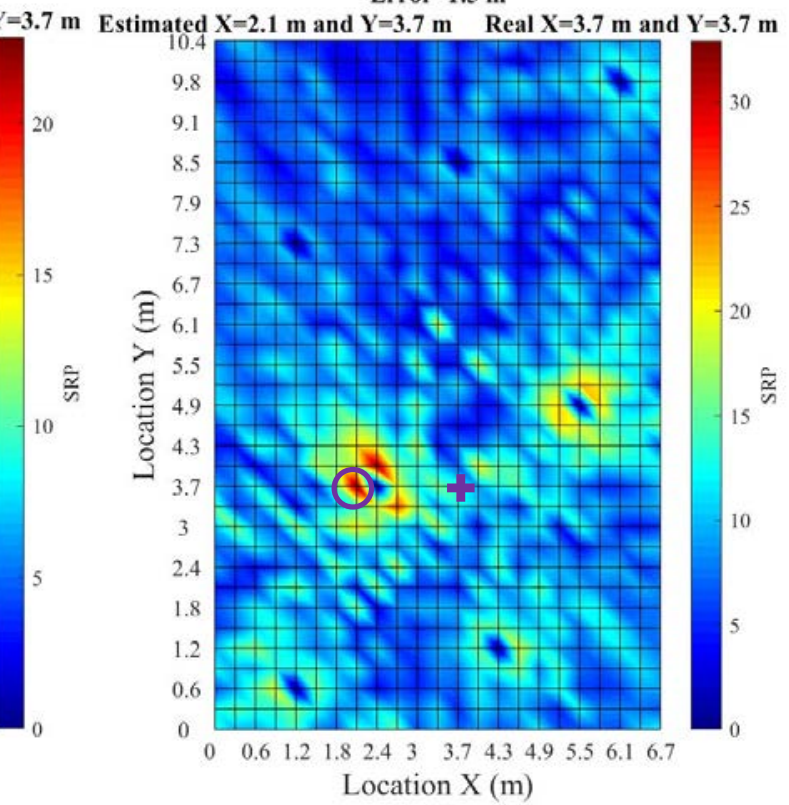

(d) $b=0.75$ 


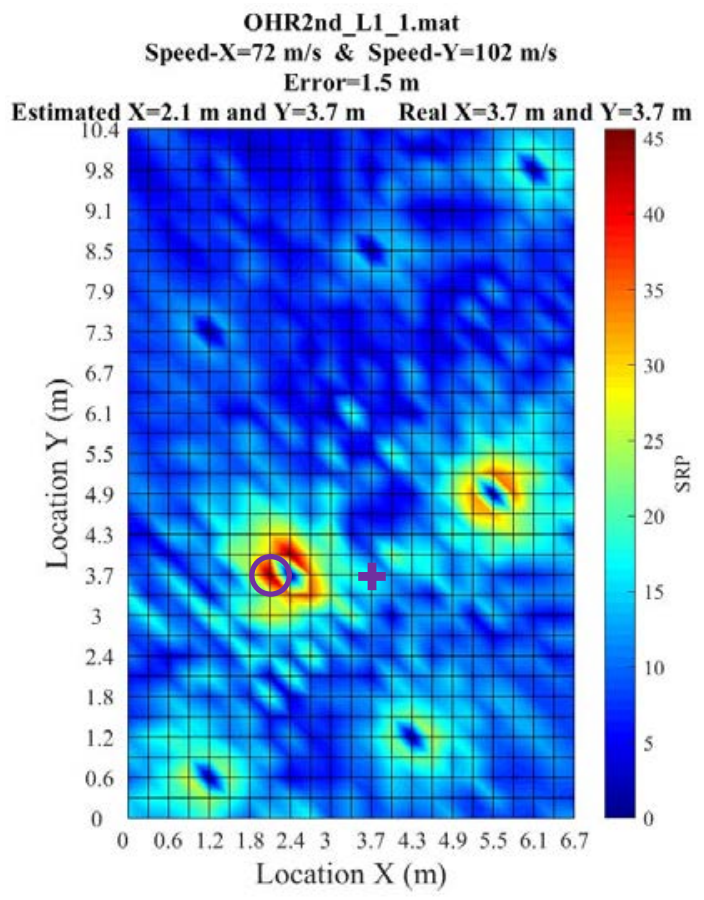

(e) $b=1.0$

Figure 3.41: Effect of Decaying Power, $b$, Versus Inverse Distance Weighting on the SRP Plot

In relation to Figure 3.41, Table 3.26 shows the contribution of each accelerometer at the candidate point location with coordinates $\mathrm{X}=3.7 \mathrm{~m}$ and $\mathrm{Y}=3.7 \mathrm{~m}$. The bottom row of the table shows the localization error for each decaying power. When the decaying power was 0.0 and 0.25 (Figures 3.41(a) and 3.41(b)), the localization errors were $2.5 \mathrm{~m}$, and the corresponding figures were ambiguous due to the presence of multiple peaks with close steered power values. However, a decaying power of 0.5 (Figure 3.41(c)) resulted in a $1.5 \mathrm{~m}$ localization error, and the figure associated with it was less ambiguous. Although decaying powers of $0.75,1.0$, and higher achieved a $1.5 \mathrm{~m}$ localization error, Figures 3.41(d) and 3.41(e) showed excessive weighting toward the A6 and A2 accelerometers (see Table 3.26 for reference). Therefore, the maximum steered power is very close to these accelerometers. Note that excessive weighting should be avoided by visual inspection of the SRP plot. Hereafter, the decaying power associated with each SRP plot will be added to the caption of the plot. 
Table 3.26: Accelerometer Contributions with Respect to Different Decaying Powers in Equation 2.12 at Coordinates $\mathrm{X}=3.7 \mathrm{~m}$ and $\mathrm{Y}=3.7 \mathrm{~m}$ for The Test OHR2nd-L1-1

\begin{tabular}{|c|c|c|c|c|c|c|}
\hline \multirow{2}{*}{ Accelerometer } & \multirow{2}{*}{$\begin{array}{c}\boldsymbol{f} \\
(\mathbf{m})\end{array}$} & $\mathbf{0 . 0 0}$ & $\mathbf{0 . 2 5}$ & $\mathbf{0 . 5 0}$ & $\mathbf{0 . 7 5}$ & $\mathbf{1 . 0 0}$ \\
\cline { 3 - 7 } & 2.5 & $14.3 \%$ & $15.1 \%$ & $15.6 \%$ & $15.9 \%$ & $15.8 \%$ \\
\hline A1 & 2.2 & $14.3 \%$ & $15.6 \%$ & $16.7 \%$ & $17.6 \%$ & $18.1 \%$ \\
\hline A2 & 4.9 & $14.3 \%$ & $12.8 \%$ & $11.2 \%$ & $9.7 \%$ & $8.2 \%$ \\
\hline A3 & 6.6 & $14.3 \%$ & $11.9 \%$ & $9.7 \%$ & $7.7 \%$ & $6.1 \%$ \\
\hline A4 & 4.4 & $14.3 \%$ & $13.1 \%$ & $11.8 \%$ & $10.4 \%$ & $9.1 \%$ \\
\hline A5 & 1.2 & $14.3 \%$ & $18.1 \%$ & $22.4 \%$ & $27.3 \%$ & $32.6 \%$ \\
\hline A6 & 3.9 & $14.3 \%$ & $13.5 \%$ & $12.5 \%$ & $11.4 \%$ & $10.2 \%$ \\
\hline A7 & & & & & \\
\hline \multicolumn{2}{|c|}{ Localization Error } & $2.5 \mathrm{~m}$ & $2.5 \mathrm{~m}$ & $1.5 \mathrm{~m}$ & $1.5 \mathrm{~m}$ & $1.5 \mathrm{~m}$ \\
\hline
\end{tabular}

Figure 3.41 showed the SRP plot only when the WPS in the X-and Y-directions was $72 \mathrm{~m} / \mathrm{s}$ and $102 \mathrm{~m} / \mathrm{s}$. As already mentioned at the beginning of this section, the WPS in the $\mathrm{X}$ - and $\mathrm{Y}$-directions was estimated to be $72-80 \mathrm{~m} / \mathrm{s}$ and $102-113 \mathrm{~m} / \mathrm{s}$ for white noise excitation. Indeed, 108 different SRP plots were able to be generated for these speeds in $\mathrm{X}$ - and Y-directions. The accuracy of the SRP method for these WPS ranges was then investigated, and the error for each case was recorded. Figure 3.42 shows the localization error results for all 108 SRP plots. The numbers inside the figure show the SRP localization error associated with each WPS in the X-and Y-directions for the maximum steered power. Lighter colors were used for the cells with fewer localization errors. 


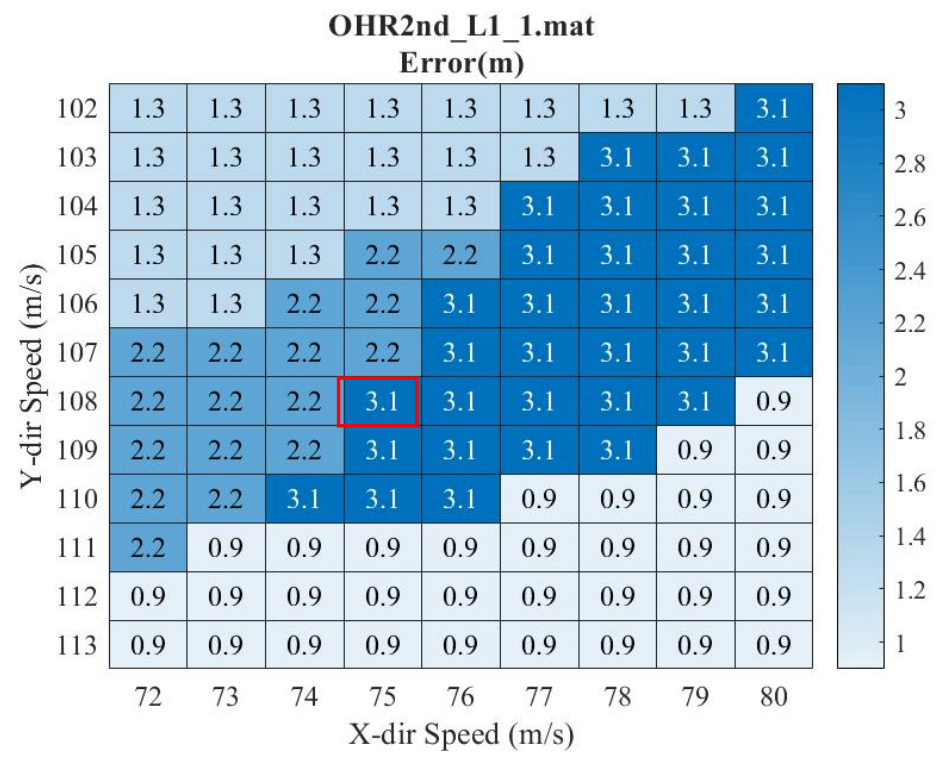

Figure 3.42: Localization Error Plot with Respect to WPS in X- and Y-Directions $(b=0.5)$

The localization error in Figure 3.42 is from $0.9 \mathrm{~m}$ to $3.1 \mathrm{~m}$ for the tested bay. Table 3.27 indicates the average of values already shown in Table 3.7 for the estimated WPS in the X- and Y-directions for white noise excitation. Based on Table 3.27, if the WPS in the $\mathrm{X}$ - and Y-directions is $75 \mathrm{~m} / \mathrm{s}$ and $108 \mathrm{~m} / \mathrm{s}$, the localization error is $3.1 \mathrm{~m}$. The error associated with the average WPS was marked by a red rectangle in Figure 3.42 and in similar figures hereafter.

Table 3.27: Average WPS in X- and Y-Directions for the White Noise Excitation Based on Table 3.7

\begin{tabular}{|c|c|c|}
\cline { 2 - 3 } \multicolumn{1}{c|}{} & $\begin{array}{c}\text { Speed25 (Shaker Power 50\%) } \\
(\mathbf{m} / \mathbf{s})\end{array}$ & $\begin{array}{c}\text { Speed26 (Shaker Power 25\%) } \\
(\mathbf{m} / \mathbf{s})\end{array}$ \\
\hline Y-Dir & 107.8 & 108.3 \\
\hline X-Dir & 74.3 & 76.2 \\
\hline
\end{tabular}

The results shown in Figure 3.42 were determined by considering the entire time duration of all signals shown in Figure 3.40. Due to the time intensiveness of the computations, it took more than one hour to generate Figure 3.42. To speed the calculation, a small segment of each signal was investigated. Figure 3.43 illustrates the time from 10.3 sec to 12.3 sec for each accelerometer, and Figure 3.44 shows the corresponding localization errors. With this change, it took less than two minutes to generate Figure 3.44. 
As previously stated, this time duration, which began immediately before the shaker started working, was selected under the assumption that the signal might contain less noise.
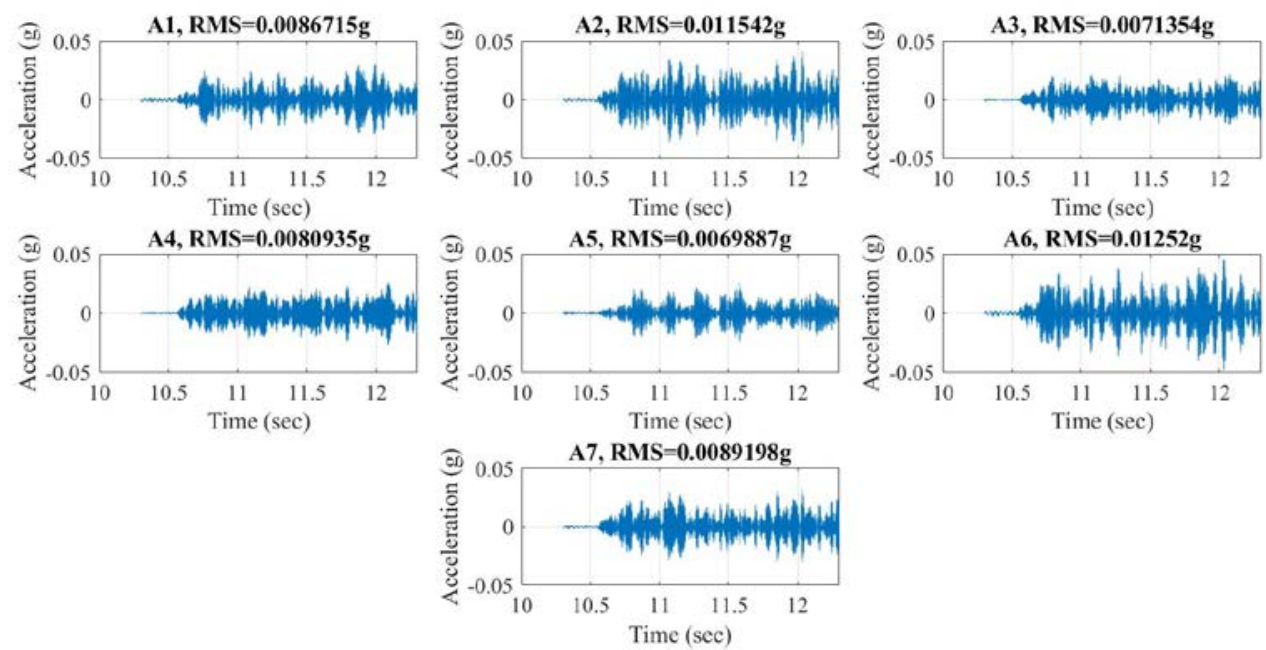

Figure 3.43: Considered Two-Second Waveform from 10.3-12.3 sec for All Accelerometers for The Test OHR2nd-L1-1

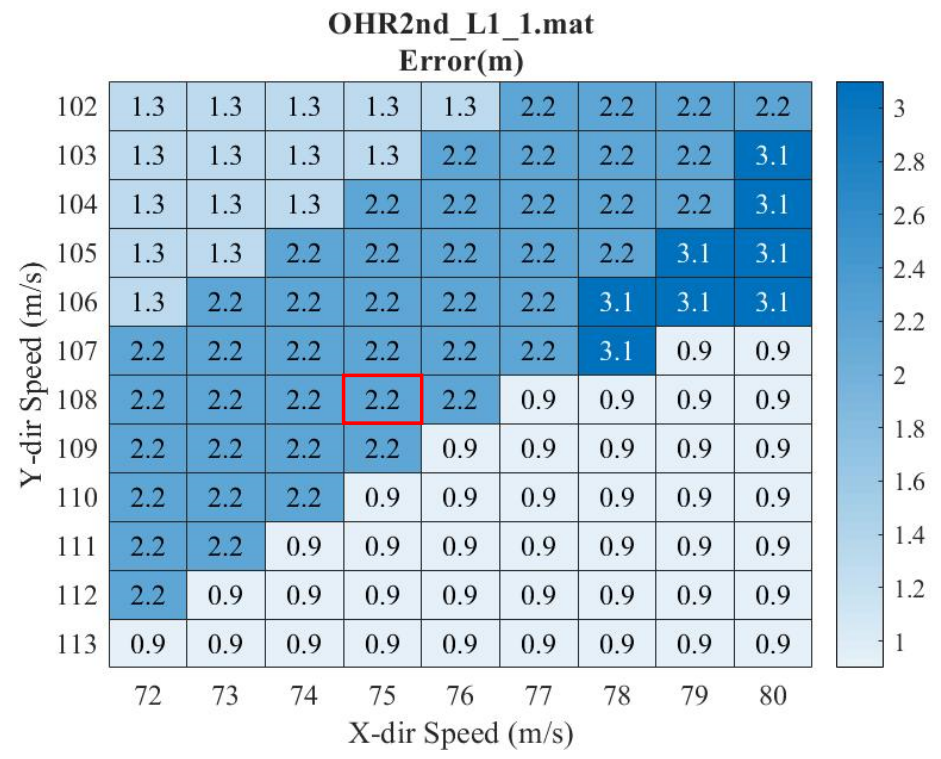

Figure 3.44: Localization Error Plot with Respect to WPS in X- and Y-Directions Considering Two-Second Waveform from 10.3-12.3 sec $(b=0.5)$

In a real-world example, we may not be able to capture the acceleration data immediately before the vibration source starts working. Therefore, as another attempt, 
Figure 3.45 shows the localization error for a five-second duration from $15 \mathrm{sec}$ to $20 \mathrm{sec}$ (five seconds after the shaker started working.)

\begin{tabular}{|c|c|c|c|c|c|c|c|c|c|c|}
\hline \multicolumn{10}{|c|}{$\begin{array}{l}\text { OHR2nd_L1_1.mat } \\
\text { Error(m) }\end{array}$} & \\
\hline 102 & 1.3 & 1.3 & 1.3 & 1.3 & 1.3 & 1.3 & 2.2 & 2.2 & 3.1 & 3 \\
\hline 103 & 1.3 & 1.3 & 1.3 & 1.3 & 2.2 & 2.2 & 2.2 & 3.1 & 3.1 & 2.8 \\
\hline 104 & 1.3 & 1.3 & 1.3 & 2.2 & 2.2 & 2.2 & 3.1 & 3.1 & 3.1 & \\
\hline 105 & 1.3 & 1.3 & 2.2 & 2.2 & 2.2 & 2.2 & 3.1 & 3.1 & 3.1 & \\
\hline 106 & 1.3 & 2.2 & 2.2 & 2.2 & 2.2 & 3.1 & 3.1 & 3.1 & 3.1 & \\
\hline d.d 107 & 2.2 & 2.2 & 2.2 & 2.2 & 2.2 & 3.1 & 3.1 & 3.1 & 3.1 & \\
\hline مै 108 & 2.2 & 2.2 & 2.2 & 2.2 & 3.1 & 3.1 & 3.1 & 3.1 & 0.9 & \\
\hline 109 & 2.2 & 2.2 & 2.2 & 3.1 & 3.1 & 3.1 & 3.1 & 0.9 & 0.9 & \\
\hline 110 & 2.2 & 2.2 & 2.2 & 3.1 & 3.1 & 0.9 & 0.9 & 0.9 & 0.9 & \\
\hline 111 & 2.2 & 2.2 & 0.9 & 0.9 & 0.9 & 0.9 & 0.9 & 0.9 & 0.9 & 4 \\
\hline 112 & 2.2 & 0.9 & 0.9 & 0.9 & 0.9 & 0.9 & 0.9 & 0.9 & 0.9 & 1.2 \\
\hline 113 & 0.9 & 0.9 & 0.9 & 0.9 & 0.9 & 0.9 & 0.9 & 0.9 & 0.9 & 1 \\
\hline & 72 & 73 & 74 & $\begin{array}{r}75 \\
\text { X-dir }\end{array}$ & $\begin{array}{c}76 \\
\text { Spee }\end{array}$ & $\begin{array}{c}77 \\
(\mathrm{~m} / \mathrm{s})\end{array}$ & 78 & 79 & 80 & \\
\hline
\end{tabular}

Figure 3.45: Localization Error Plot with Respect to WPS in X- and Y-Directions Considering Five-Second Waveform from 15-20 sec $(b=0.5)$

There is no significant improvement between Figures 3.44 and 3.45, and some equal values are repeated diagonally in these two figures. Figure 3.46 shows the SRP plots for specific speeds corresponding to Figures 3.44 and 3.45. The localization errors of the three highest steered powers are also indicated. 


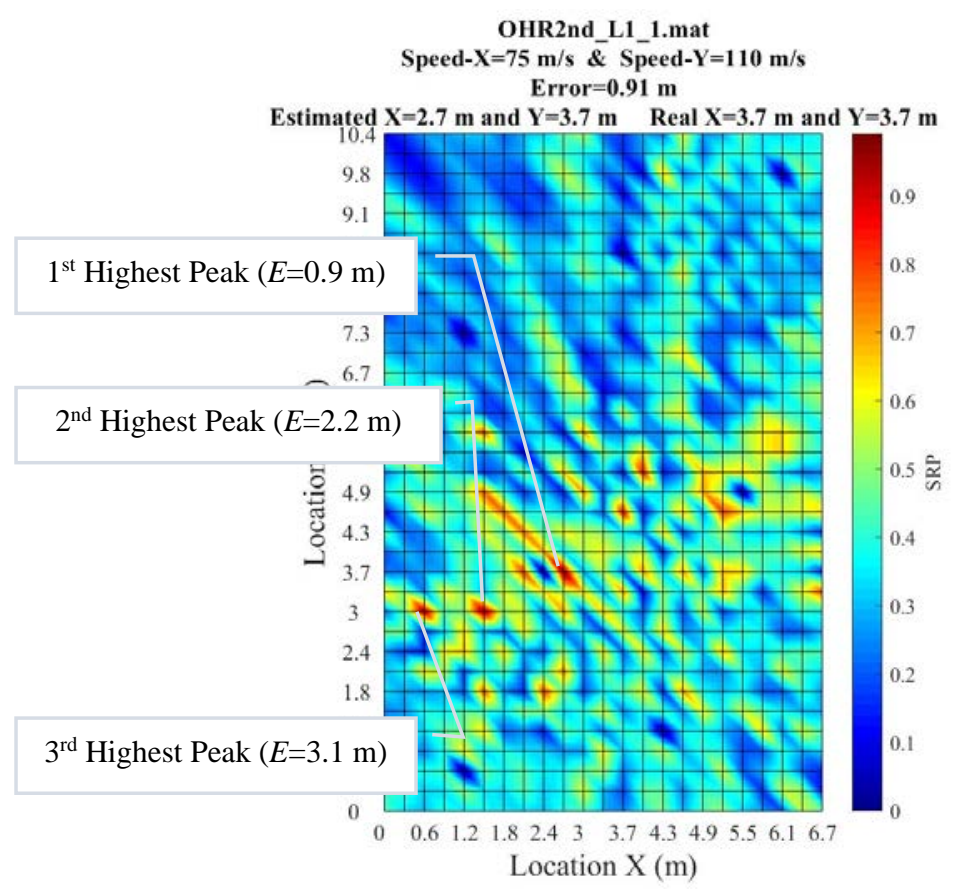

(a) Waveform from 10.3-12.3 sec

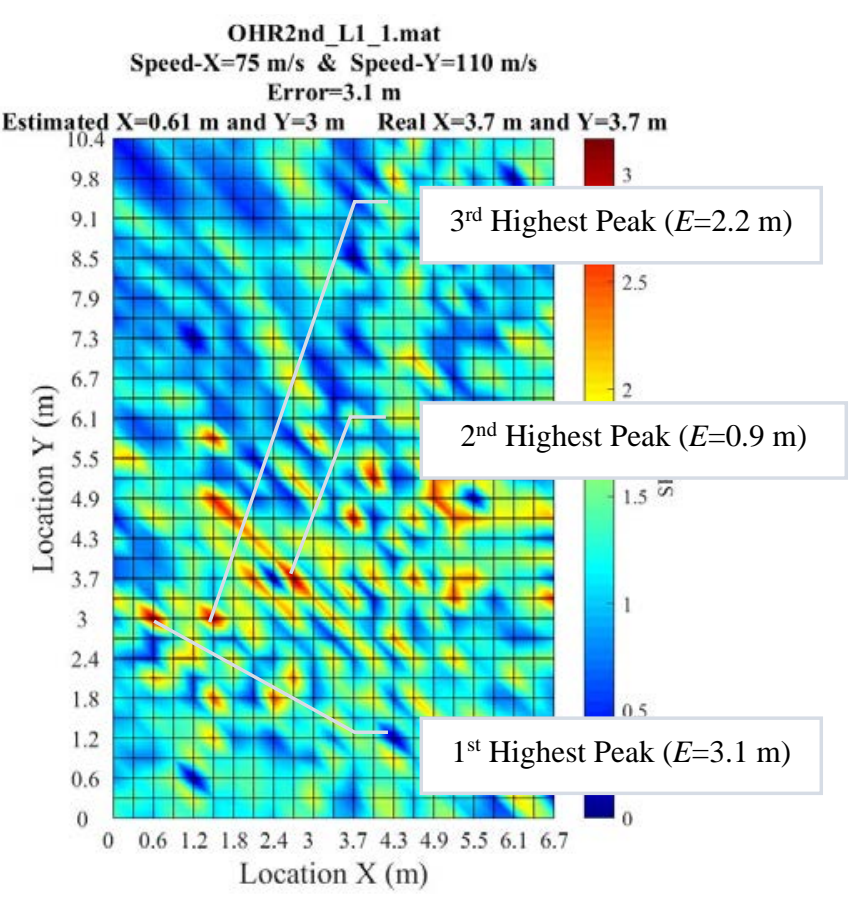

(b) Waveform from $15-20 \mathrm{sec}$

Figure 3.46: Effect of Length of Time Duration on the SRP Plots $(b=0.5)$

In Figure 3.46, the overall shape of the SRP plots is almost the same, but the order of the peaks is different. The first highest peak in Figure 3.46(a) is equivalent to the second highest peak in Figure 3.46(b). Fortunately, Figures 3.44 and 3.45 show only four different localization errors (i.e., $0.9 \mathrm{~m}, 1.3 \mathrm{~m}, 2.2 \mathrm{~m}$, and $3.1 \mathrm{~m}$ ). It was previously mentioned that the localization errors are shown in Figures 3.44, and 3.45 refer only to the first highest steered power. Because the errors corresponding to the maximum steered power may not necessarily represent the most likely source, it is helpful to see the localization error corresponding to the first three highest peaks.

Moreover, although the localization error plot only shows the error for the first highest peak, it is nevertheless the best way to chart the localization errors with respect to the different WPS in the X- and Y-directions.

So far, the use of inverse distance weighting has reduced the localization error significantly. Furthermore, examining a small segment of the signal instead of the entire time-domain made the localization processing almost 30 times faster without substantially affecting the accuracy. Therefore, in the rest of this section, the time duration from 15 to 
17 seconds will be considered in the post-processing. The filtering technique was used as another attempt to reduce the localization error. As Figure 3.47 shows, the signals were filtered in frequency ranges of 5-200 Hz and $80-180 \mathrm{~Hz}$, separately. The type of filter utilized for this purpose was the Butterworth IIR filter, and it was set to an order of 3.0.

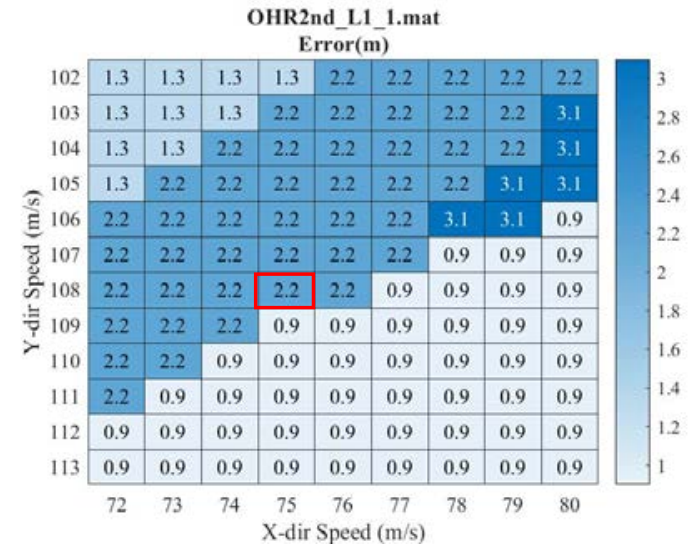

(a) Filtering in Range of $5-200 \mathrm{~Hz}$

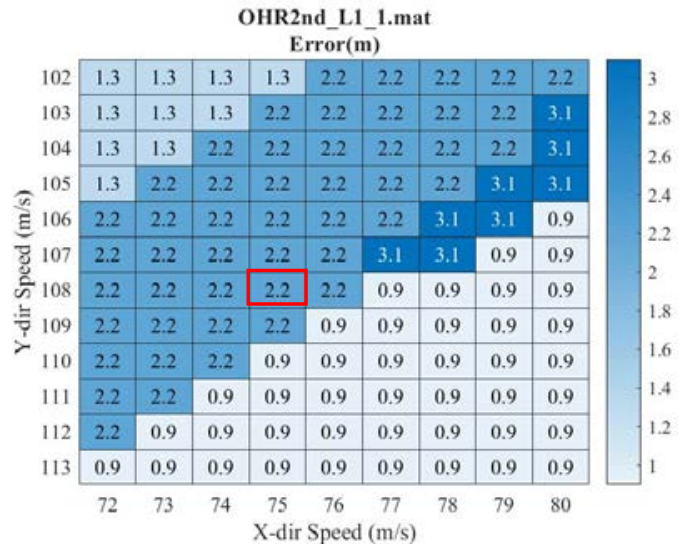

(b) Filtering in Range of $80-180 \mathrm{~Hz}$

Figure 3.47: Effect of Filtering on Localization Error Plots with Respect to WPS in Xand Y-Directions Considering Two-Second Waveform from 15-17 sec $(b=0.5)$

As illustrated in Figures 3.47(a) and 3.47(b), the use of filtering did not improve the results significantly. All the localization errors discussed above were for test OHR2ndL1-1. The localization error for test OHR2nd-L1-2 is shown in Figure 3.48. 


\begin{tabular}{|c|c|c|c|c|c|c|c|c|c|c|}
\hline \multicolumn{10}{|c|}{$\begin{array}{l}\text { OHR2nd_L1_2.mat } \\
\operatorname{Error}(m)\end{array}$} & \\
\hline 102 & 1.3 & 1.3 & 1.3 & 1.3 & 1.3 & 2.2 & 2.2 & 2.2 & 1.7 & \\
\hline 103 & 1.3 & 1.3 & 1.3 & 1.3 & 2.2 & 2.8 & 2.2 & 1.7 & 1.7 & 2.6 \\
\hline 104 & 1.3 & 1.3 & 1.3 & 2.2 & 1.7 & 1.7 & 1.7 & 1.7 & 1.7 & 2.4 \\
\hline 105 & 1.7 & 1.7 & 1.7 & 1.7 & 1.7 & 1.7 & 1.7 & 1.7 & 1.7 & 2 \\
\hline 106 & 1.7 & 1.7 & 1.7 & 1.7 & 1.7 & 1.7 & 1.7 & 1.7 & 1.7 & \\
\hline 107 & 1.7 & 1.7 & 1.7 & 1.7 & 1.7 & 1.7 & 1.7 & 1.7 & 1.7 & \\
\hline 108 & 1.7 & 1.7 & 1.7 & 1.7 & 1.7 & 1.7 & 1.7 & 1.7 & 1.7 & 1.8 \\
\hline 109 & 1.7 & 1.7 & 1.7 & 1.7 & 1.7 & 1.7 & 1.7 & 1.7 & 1.7 & 1.6 \\
\hline 110 & 1.6 & 1.6 & 1.6 & 2.8 & 2.8 & 1.7 & 0.9 & 0.9 & 0.9 & \\
\hline 111 & 2.2 & 1.6 & 2.8 & 2.8 & 2.8 & 0.9 & 0.9 & 0.9 & 0.9 & \\
\hline 112 & 2.2 & 0.9 & 2.8 & 2.8 & 0.9 & 0.9 & 0.9 & 0.9 & 0.9 & 1.2 \\
\hline 113 & 0.9 & 0.9 & 0.9 & 0.9 & 0.9 & 0.9 & 0.9 & 0.9 & 0.9 & 1 \\
\hline & 72 & 73 & 74 & $\begin{array}{r}75 \\
\text { X-d }\end{array}$ & $\begin{array}{r}76 \\
\text { Spee }\end{array}$ & $\begin{array}{c}77 \\
(\mathrm{~m} / \mathrm{s})\end{array}$ & 78 & 79 & 80 & \\
\hline
\end{tabular}

Figure 3.48: Localization Error Plot with Respect to WPS in X- and Y-Directions when Considering a Two-Second Waveform from 15-17 sec and Filtering in a Frequency Range of $5-200 \mathrm{~Hz}(b=0.5)$

Figure 3.49 shows the SRP plots for tests in which the shaker was placed at L1. The shaker power for tests OHR2nd_L1_1 and OHR2nd_L1_2 was 50\% and 25\%, respectively. Note that the use of higher decaying power, i.e., $b$, did not improve the quality of the SRP plot. 


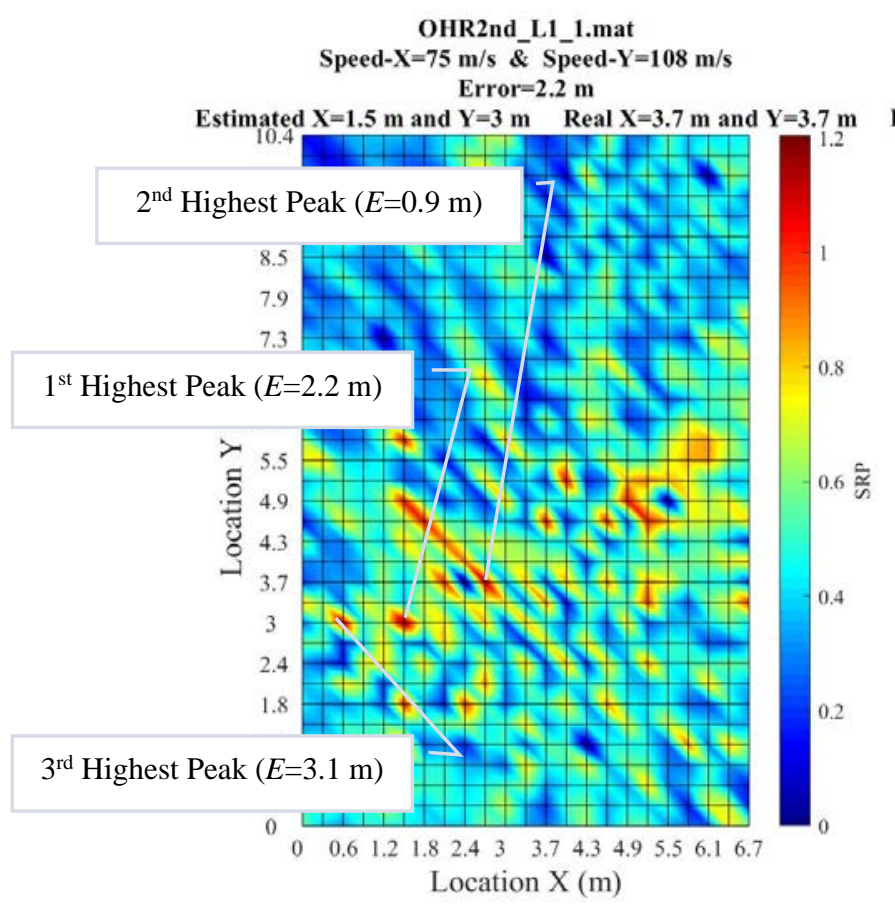

(a) OHR2nd-L1-1

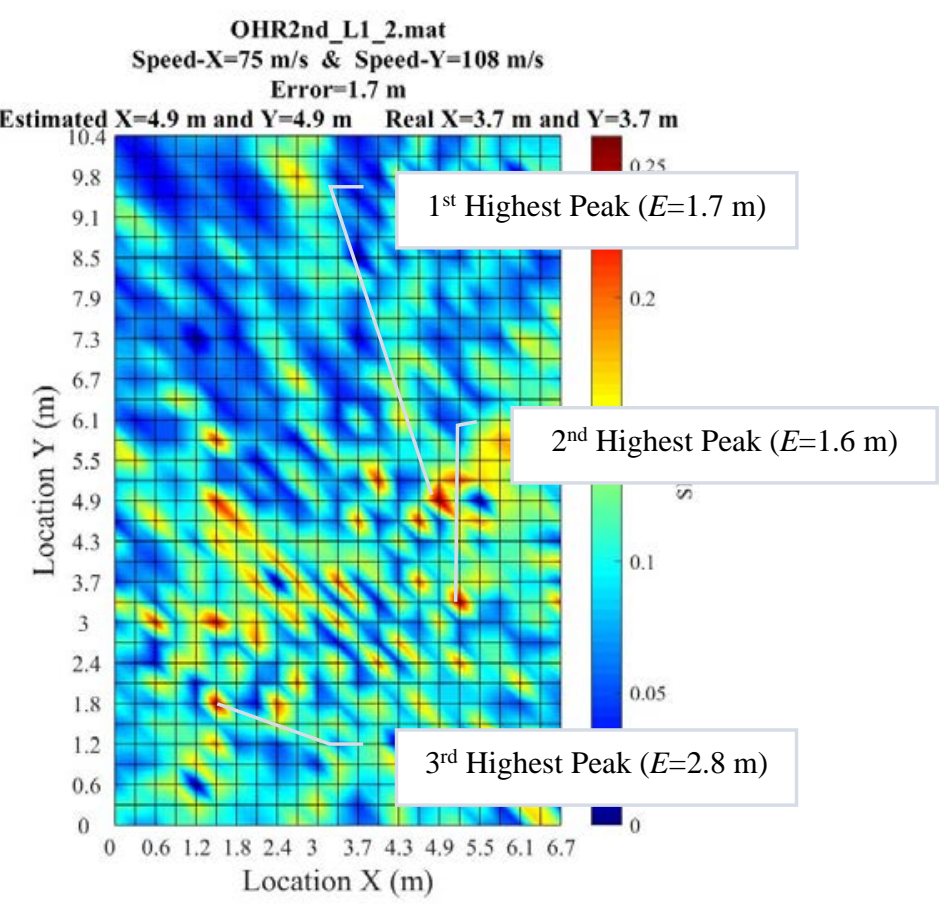

(b) OHR2nd-L1-2

Figure 3.49: SRP Plots when Considering a Two-Second Waveform from 15-17 sec and Filtering in a Frequency Range of $5-200 \mathrm{~Hz}(b=0.5)$

The results for the rest of the tests (listed in Table 3.25) are shown in Appendix A.1.

In order to summarize the SRP performance in relation to white noise excitation, Table 3.28 lists the tests and their corresponding localization errors for the three highest steered powers.

Table 3.28: Localization Errors for the First, Second, and Third Highest Peaks When the WPS in X- and Y-Directions is $75 \mathrm{~m} / \mathrm{s}$ and $108 \mathrm{~m} / \mathrm{s}$

\begin{tabular}{|c|c|c|c|c|}
\hline \multirow[b]{2}{*}{ Test Name } & \multirow{2}{*}{$\begin{array}{l}\text { White Noise } \\
\text { Excitation }\end{array}$} & \multicolumn{3}{|c|}{ Error Corresponding to Peaks } \\
\hline & & $\begin{array}{c}1^{\text {st }} \text { Highest } \\
(\mathrm{m})\end{array}$ & $\begin{array}{c}2^{\text {nd }} \text { Highest } \\
(\mathrm{m})\end{array}$ & $\begin{array}{l}3^{\text {rd }} \text { Highest } \\
\text { (m) }\end{array}$ \\
\hline OHR2nd_L1_1 & $5 \mathrm{~Hz}-200 \mathrm{~Hz}$ & 2.2 & 0.9 & 3.1 \\
\hline OHR2nd_L1_2 & $5 \mathrm{~Hz}-200 \mathrm{~Hz}$ & 1.7 & 1.6 & 2.8 \\
\hline OHR2nd_L2_1 & $5 \mathrm{~Hz}-200 \mathrm{~Hz}$ & 1.6 & 2.2 & 1.9 \\
\hline OHR2nd_L2_2 & $5 \mathrm{~Hz}-200 \mathrm{~Hz}$ & 1.6 & 2.2 & 1.9 \\
\hline OHR2nd_L3_1 & $5 \mathrm{~Hz}-200 \mathrm{~Hz}$ & 0.9 & 1.6 & 2.3 \\
\hline OHR2nd_L3_2 & $5 \mathrm{~Hz}-200 \mathrm{~Hz}$ & 0.9 & 1.6 & 2.3 \\
\hline OHR2nd_L4_1 & $5 \mathrm{~Hz}-200 \mathrm{~Hz}$ & 4.5 & 3 & 3.1 \\
\hline OHR2nd_L4_2 & $5 \mathrm{~Hz}-200 \mathrm{~Hz}$ & 4.5 & 2.8 & 3 \\
\hline
\end{tabular}




\subsubsection{Localization of Sinusoid Excitation}

Section 3.6.1 discussed the performance of the SRP method with regard to white noise excitation. In this section, the SRP method was assessed using three sinusoid excitations. As mentioned previously, machinery-induced vibrations are most likely to generate sinusoid excitation. Some rotating machinery, e.g., gears, pumps, turbines, may work with different speeds, causing different vibration frequencies. If the generated vibration frequency is close enough to the natural frequency of the floor, the floor will be likely to have vibration problems. Therefore, the frequencies of $70 \mathrm{~Hz}, 90 \mathrm{~Hz}$, and $110 \mathrm{~Hz}$ were chosen for testing sinusoid excitation on the tested floor. The results will be presented in the following three sections. As specified in Table 3.23, the shaker was placed at either the L1, L2, L3, or L4 location in these tests. The setup of each test can be viewed in figures 3.39, A.1, A.4, and A.7. Finally, the locations of the accelerometers in all the tests have already been shown in Table 3.24.

\subsubsection{70 Hz Sinusoid Excitation}

The sinusoid frequency of vibration excitation used in this section is $70 \mathrm{~Hz}$. Table 3.29 shows the individual test names and their corresponding shaker power.

Table 3.29: List of Tests with $70 \mathrm{~Hz}$ Sinusoid Excitation

\begin{tabular}{|c|c|c|c|c|}
\hline Test Name & $\begin{array}{c}\text { Shaker } \\
\text { Location }\end{array}$ & Excitation & $\begin{array}{c}\text { Frequency Range } \\
\mathrm{Hz}\end{array}$ & $\begin{array}{l}\text { Shaker } \\
\text { Power }\end{array}$ \\
\hline OHR2nd-L1-6 & \multirow{2}{*}{ L1 } & \multirow{2}{*}{ Sinusoid } & \multirow{2}{*}{70} & $50 \%$ \\
\hline OHR2nd-L1-7 & & & & $75 \%$ \\
\hline OHR2nd-L2-6 & \multirow{2}{*}{ L2 } & \multirow{2}{*}{ Sinusoid } & \multirow{2}{*}{70} & $50 \%$ \\
\hline OHR2nd-L2-7 & & & & $75 \%$ \\
\hline OHR2nd-L3-5 & \multirow{2}{*}{ L3 } & \multirow{2}{*}{ Sinusoid } & \multirow{2}{*}{70} & $50 \%$ \\
\hline OHR2nd-L3-6 & & & & $75 \%$ \\
\hline OHR2nd-L4-5 & \multirow{2}{*}{ L4 } & \multirow{2}{*}{ Sinusoid } & \multirow{2}{*}{70} & $50 \%$ \\
\hline OHR2nd-L4-6 & & & & $75 \%$ \\
\hline
\end{tabular}

The WPS for the $70 \mathrm{~Hz}$ sinusoid excitation was already estimated in Section 3.5.1.2, as shown in Table 3.13. In sum, the WPS estimated a range of 35-39 m/s in the X-direction and a range of 59-62 m/s in the Y-direction. Table 3.30 shows the average WPS. 
Table 3.30: Average WPS in X- and Y-Directions for the $70 \mathrm{~Hz}$ Sinusoid Excitation

\begin{tabular}{|c|c|c|}
\cline { 2 - 3 } \multicolumn{1}{c|}{} & $\begin{array}{c}\text { Speed29 (Shaker Power 50\%) } \\
(\mathbf{m} / \mathbf{s})\end{array}$ & $\begin{array}{c}\text { Speed30 (Shaker Power 25\%) } \\
\mathbf{( m / s )}\end{array}$ \\
\hline Y-Dir & 60.8 & 60.8 \\
\hline X-Dir & 36.7 & 37.6 \\
\hline
\end{tabular}

The received acceleration response for the test OHR2nd-L1-7 is shown in Figure 3.50 .
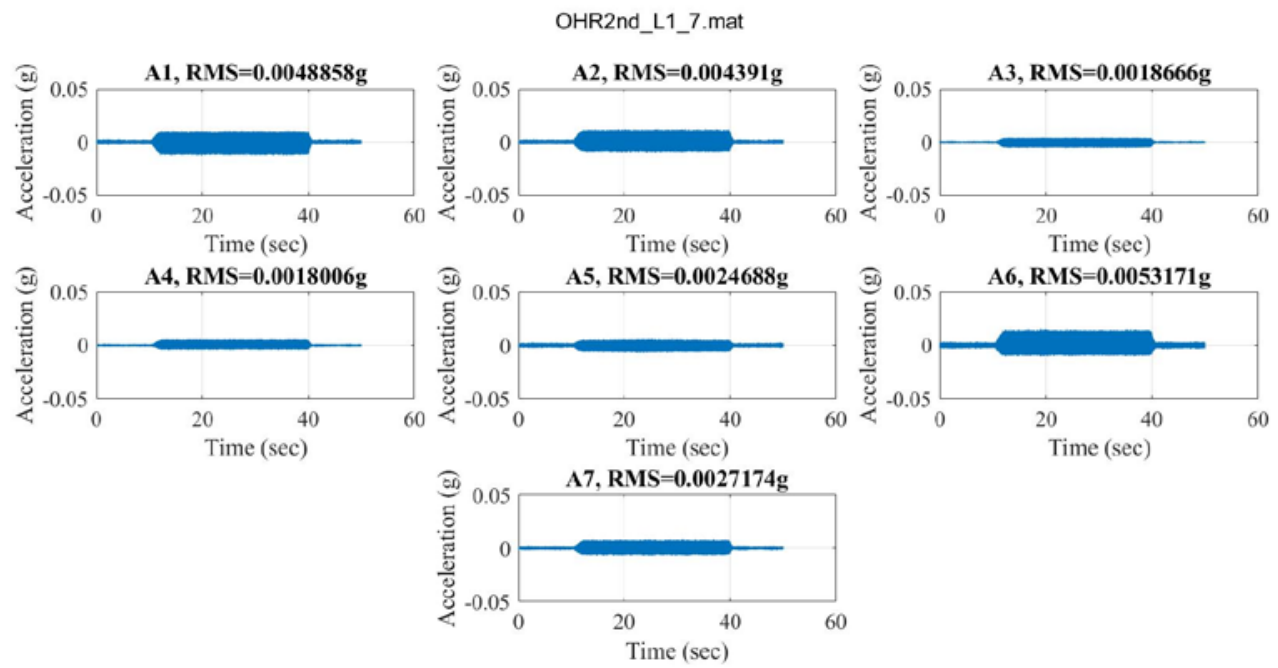

Figure 3.50: Waveforms for All Accelerometers for Test OHR2nd-L1-7

It was already explained in Chapter 2 (Equation 2.12) and Section 3.6.1 that the use of inverse distance weighting could reduce the localization error significantly in the SRP method. Figures 3.51(a) and 3.51(b) show the corresponding localization error when the decaying power, $b$, in Equation 2.12 is 0.0 and 0.5 , respectively. 


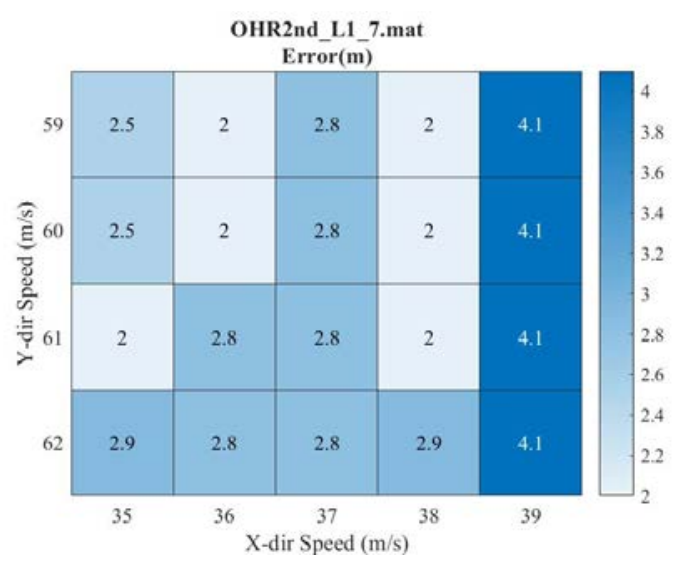

(a) $b=0$ (Equal Contribution)

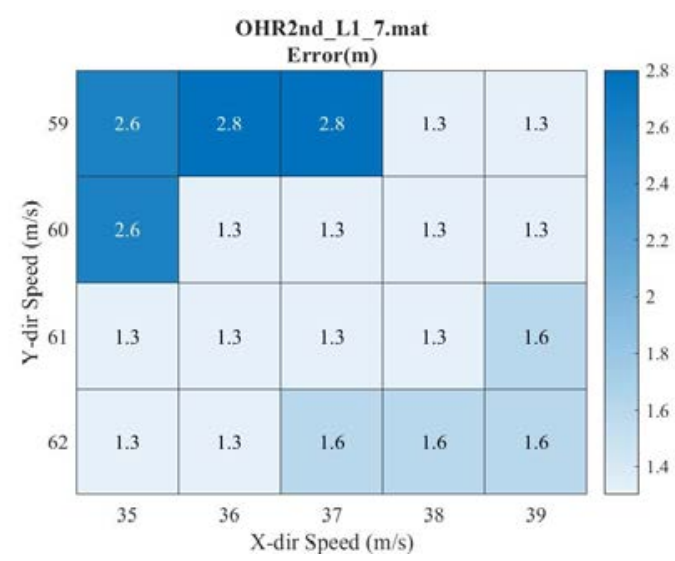

(b) $b=0.5$

Figure 3.51: The Effect of Inverse Distance Weighting on Localization Error When Considering a Two-Second Waveform from 15-17 sec $(b=0.5)$

Note that the errors shown in Figure 3.51 correspond to the maximum steered power. The SRP results for various decaying powers are indicated in Figure 3.52. The localization errors when $b$ was 0.5 or more (Figures 3.52(c)-3.52(e)) were the same. By visual inspection, $b=0.5$ looks reasonable in this SRP plot; however, there are still some peaks in the figure, making it hard to decide if the peak corresponding to the highest steered power shows the most likely source or not. In contrast, it is clear that $b=1.0$ (Figure 3.52(e)) causes more weight for the A1 and A6 accelerometers. Thus, these two accelerometers are surrounded mainly by red. It will be explained below why the estimated source locations for the second and third highest peaks and their corresponding errors may need to be visually checked. 


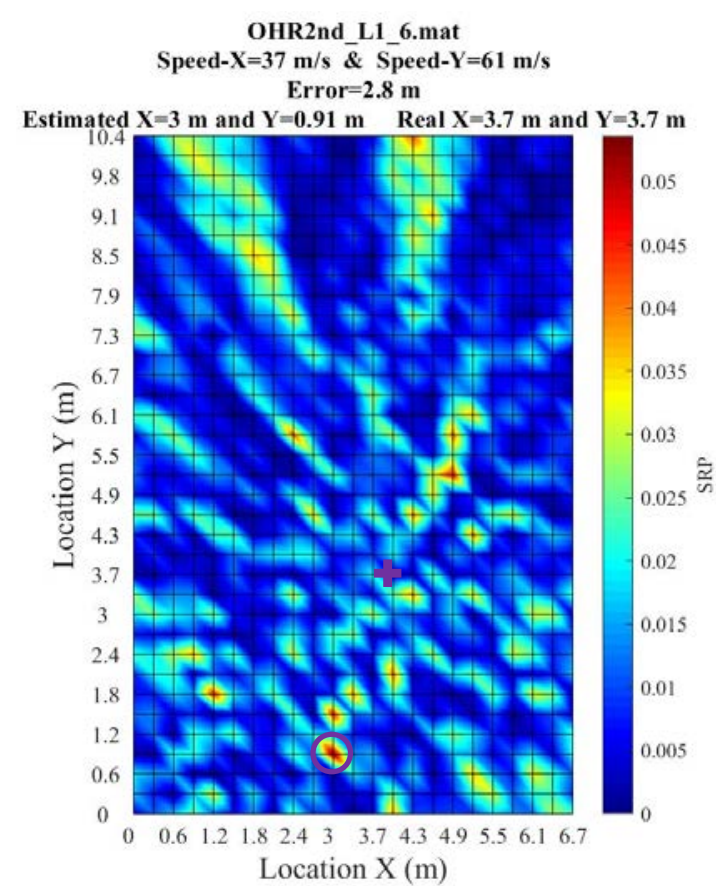

(a) $b=0$ (Equal Contribution)

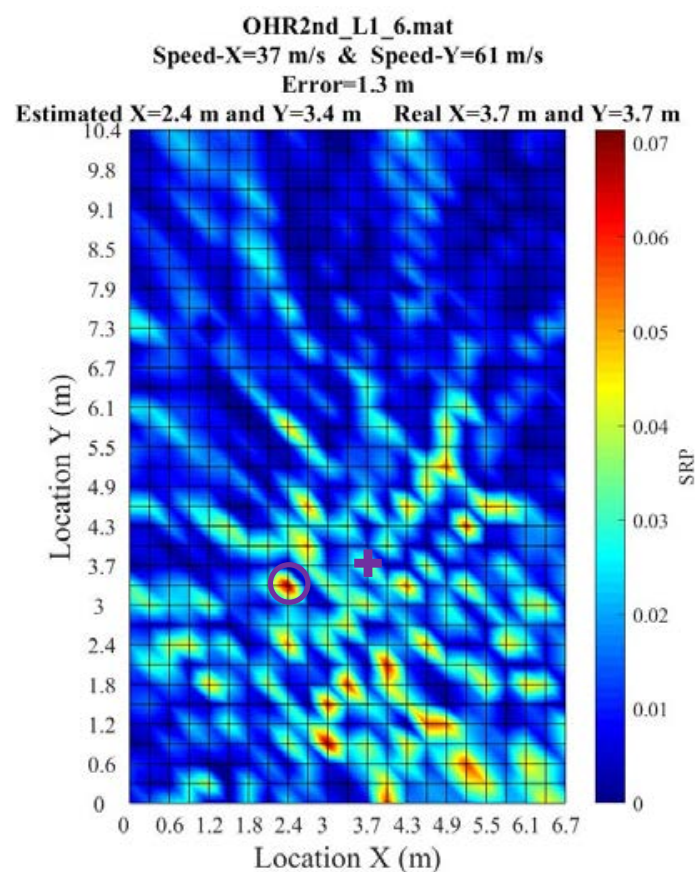

(c) $b=0.5$
OHR2nd L1 6.mat

Speed-X=37 m/s \& Speed-Y=61 m/s

Error $=2.8 \mathrm{~m}$

Estimated $X=3 \mathrm{~m}$ and $Y=0.91 \mathrm{~m} \quad$ Real $X=3.7 \mathrm{~m}$ and $Y=3.7 \mathrm{~m}$

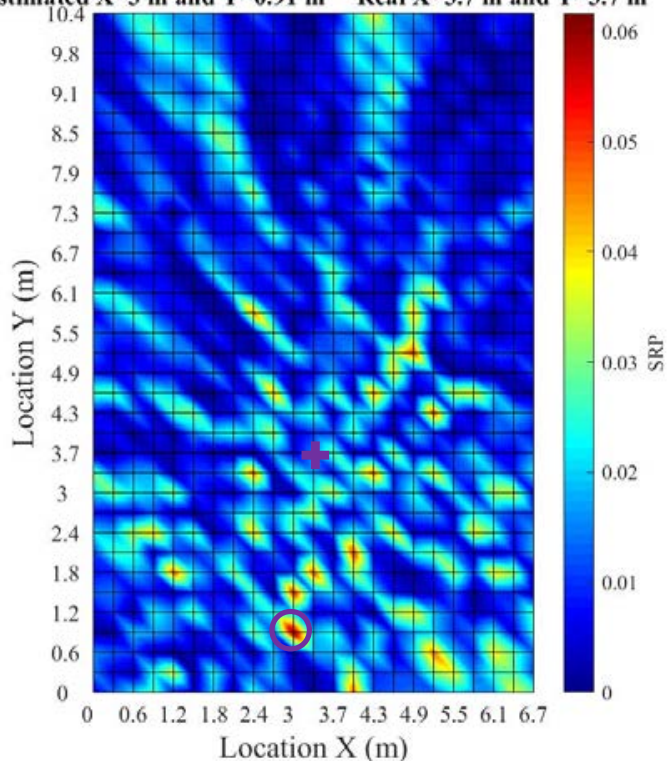

(b) $b=0.25$

\section{OHR2nd_L1_6.mat \\ Speed-X=37 m/s \& Speed- $Y=61 \mathrm{~m} / \mathrm{s}$ \\ Error $=1.3 \mathrm{~m}$}

Estimated $X=2.4 \mathrm{~m}$ and $Y=3.4 \mathrm{~m} \quad$ Real $X=3.7 \mathrm{~m}$ and $\mathrm{Y}=3.7 \mathrm{~m}$

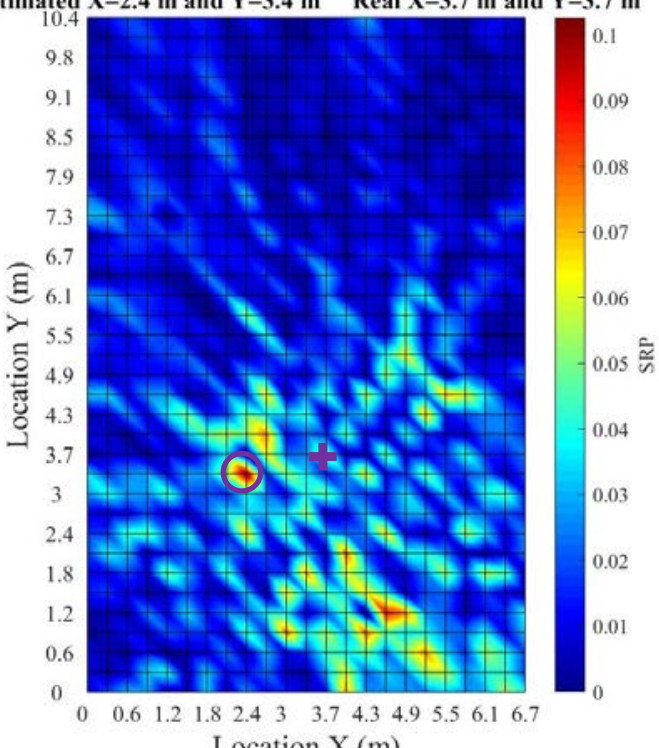

Location X (m)

(d) $b=0.75$ 


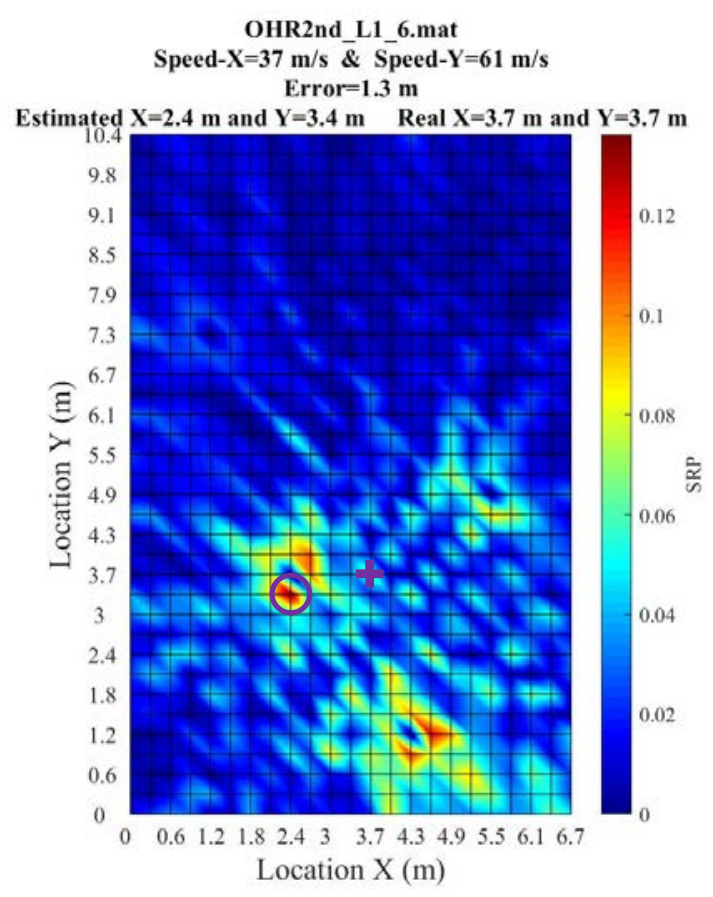

(e) $b=1.0$

Figure 3.52: Effect of Inverse Distance Weighting in the SRP Plot When Considering a Two-Second Waveform from 15-17 sec

The Butterworth filter was used with a frequency range of $60 \mathrm{~Hz}$ to $80 \mathrm{~Hz}$ to investigate the effect of filtering on the SRP plot, and the result is shown in Figure 3.53. Figures 3.53(a) and 3.53(b) illustrate that filtering had minimal effects on the SRP plot. This may be because the tests were conducted after midnight, and there was no extraneous noise present. 


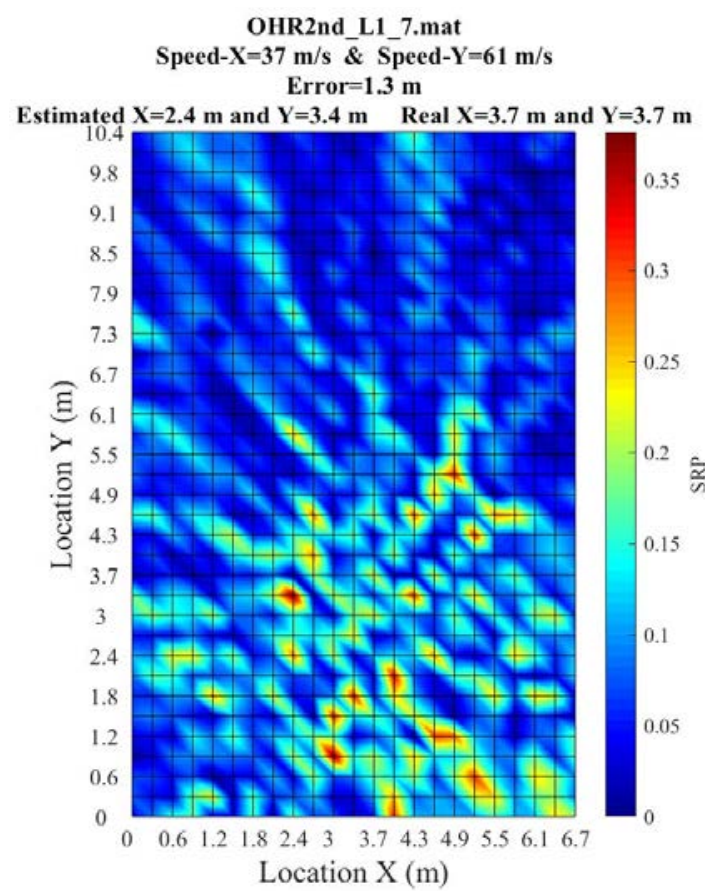

(a) With No Filter

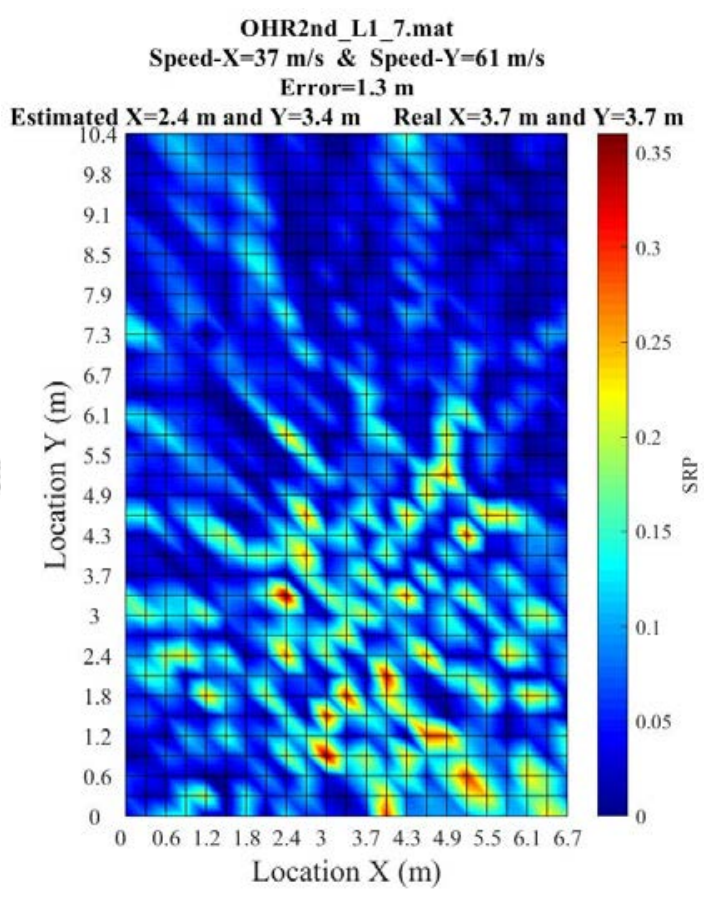

(b) Filtered in Range of $60 \mathrm{~Hz}$ to $80 \mathrm{~Hz}$

Figure 3.53: Effect of Filtering on The SRP Plot $(b=0.5)$

The SRP plots for the tests where the shaker was placed at L1 are shown in Figure 3.54. The errors which correspond to the three highest peaks are also shown there. Figure 3.55 shows only the localization error that corresponds to the highest peak. 


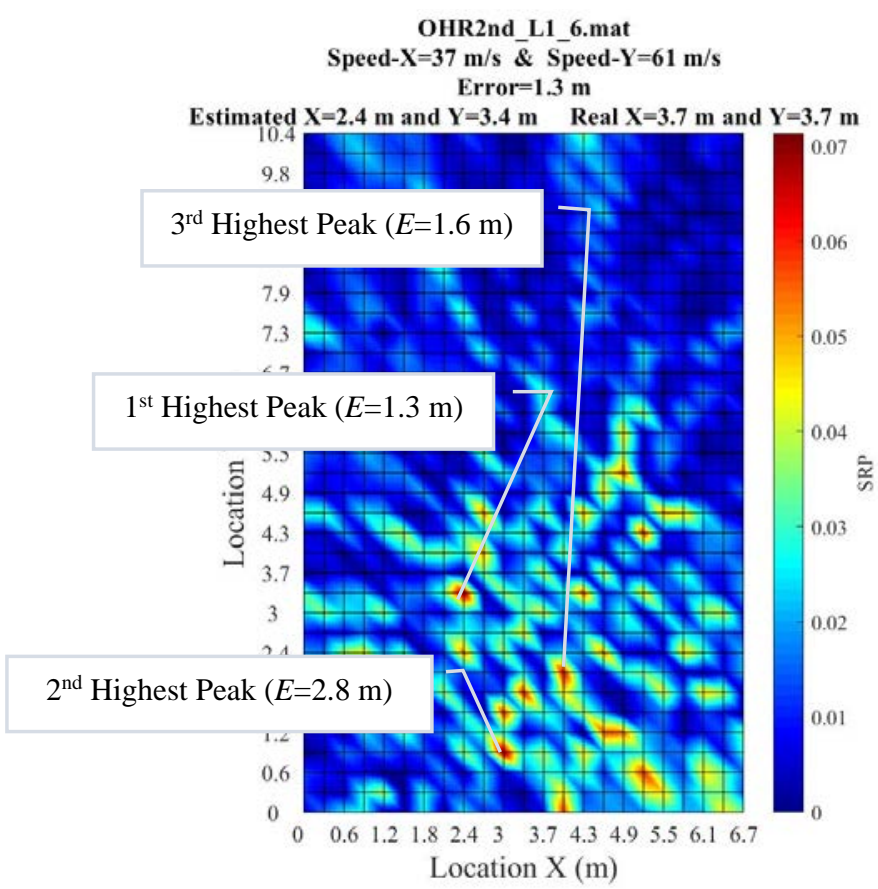

(a) Test OHR2nd-L1-6

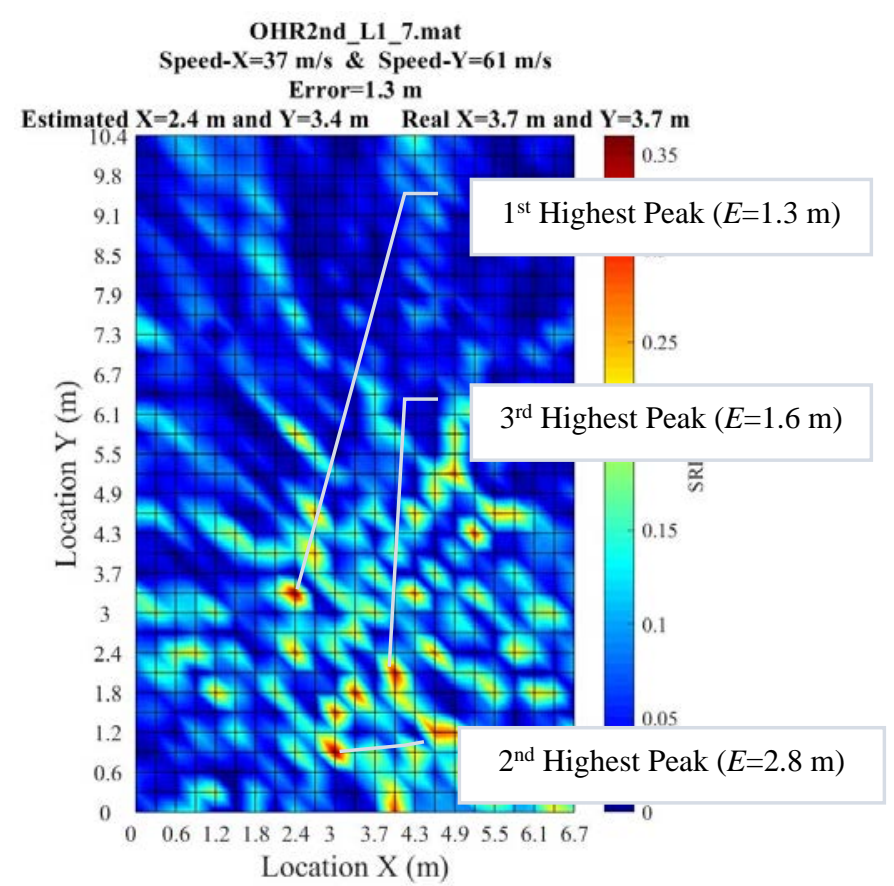

(b) Test OHR2nd-L1-7

Figure 3.54: SRP Plots When Considering a Two-Second Waveform from 15-17 sec and Filtering in a Frequency Range of $60-80 \mathrm{~Hz}(b=0.5)$

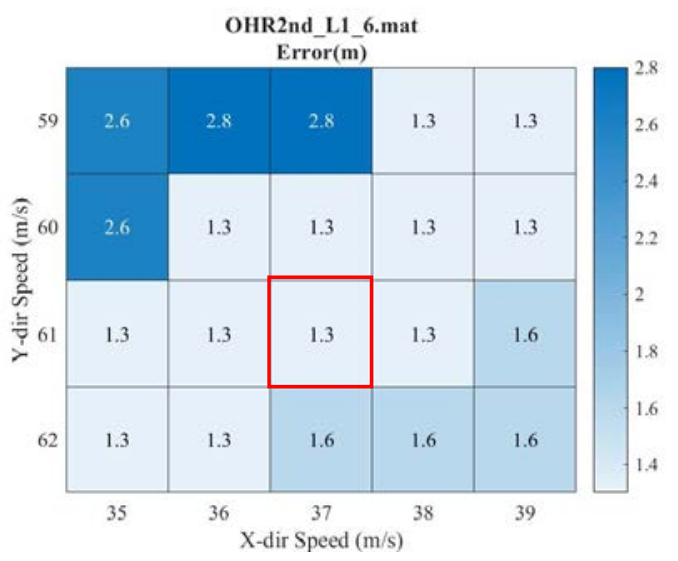

(a) Test OHR2nd-L1-6

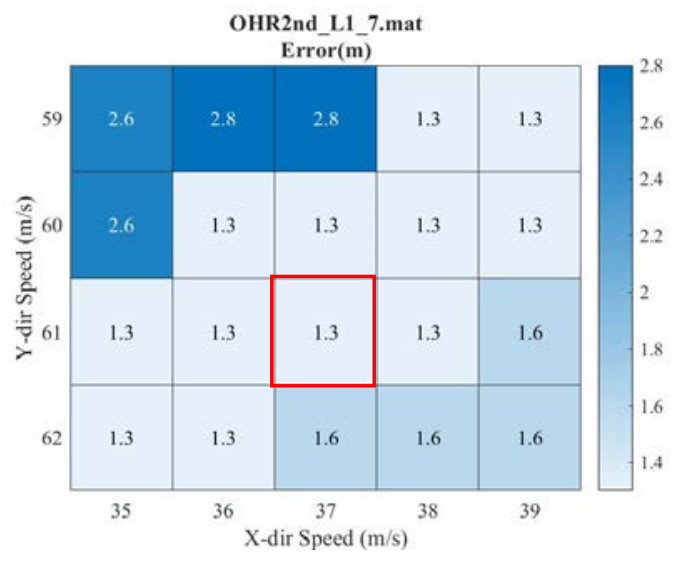

(b) Test OHR2nd-L1-7

Figure 3.55: Localization Error When Considering a Two-Second Waveform from 15-17 sec and Filtering in a Frequency Range of $60-80 \mathrm{~Hz}(b=0.5)$

The SRP plots and corresponding localization errors for the rest of the tests (listed in Table 3.29) when the shaker was placed at L2, L3, and L4 are shown in Appendix A.2. 
As mentioned previously, the SRP plots for a $70 \mathrm{~Hz}$ sinusoid excitation show more peaks than the white noise excitation, perhaps due to a periodic excitation force. Therefore, the localization errors associated with the second and third highest peaks for each test were recorded, as shown in Table 3.31. The minimum errors for each test are highlighted in green.

Table 3.31: Localization Errors for the First, Second, and Third Highest Peaks When the WPS in X- and Y-Directions is $37 \mathrm{~m} / \mathrm{s}$ and $61 \mathrm{~m} / \mathrm{s}$

\begin{tabular}{|c|c|c|c|c|}
\hline \multirow{2}{*}{ Test Name } & \multirow{2}{*}{$\begin{array}{c}\text { Sinusoid } \\
\text { Excitation }\end{array}$} & \multicolumn{3}{|c|}{ Error Corresponding to Peaks } \\
\cline { 3 - 5 } & & $\mathbf{1}^{\text {st }}$ Highest & $\mathbf{2}^{\text {nd }}$ Highest & $\mathbf{3}^{\text {rd }}$ Highest \\
\cline { 3 - 5 } & $\mathbf{( m )}$ & $\mathbf{( m )}$ & $\mathbf{( m )}$ \\
\hline OHR2nd_L1_6 & $70 \mathrm{~Hz}$ & 1.3 & 2.8 & 1.6 \\
\hline OHR2nd_L1_7 & $70 \mathrm{~Hz}$ & 1.3 & 2.8 & 1.6 \\
\hline OHR2nd_L2_6 & $70 \mathrm{~Hz}$ & 3.3 & 6.0 & 1.3 \\
\hline OHR2nd_L2_7 & $70 \mathrm{~Hz}$ & 3.3 & 6.0 & 1.3 \\
\hline OHR2nd_L3_5 & $70 \mathrm{~Hz}$ & 3.8 & 2.2 & 5.0 \\
\hline OHR2nd_L3_6 & $70 \mathrm{~Hz}$ & 3.8 & 2.2 & 3.7 \\
\hline OHR2nd_L3_7 & $70 \mathrm{~Hz}$ & 2.2 & 3.8 & 5.0 \\
\hline OHR2nd_L4_5 & $70 \mathrm{~Hz}$ & 5.6 & 2.9 & 2.4 \\
\hline OHR2nd_L4_6 & $70 \mathrm{~Hz}$ & 5.6 & 2.9 & 2.4 \\
\hline OHR2nd_L4_7 & $70 \mathrm{~Hz}$ & 5.6 & 2.9 & 2.4 \\
\hline
\end{tabular}

\subsubsection{2 $90 \mathrm{~Hz}$ Sinusoid Excitation}

The tests with $90 \mathrm{~Hz}$ sinusoid excitation is shown in Table 3.32.

Table 3.32: List of Tests with $90 \mathrm{~Hz}$ Sinusoid Excitation

\begin{tabular}{|c|c|c|c|c|}
\hline Test Name & $\begin{array}{c}\text { Shaker } \\
\text { Location }\end{array}$ & Excitation & $\begin{array}{c}\text { Frequency Range } \\
\mathrm{Hz}\end{array}$ & $\begin{array}{l}\text { Shaker } \\
\text { Power }\end{array}$ \\
\hline OHR2nd-L1-8 & \multirow{2}{*}{ L1 } & \multirow{2}{*}{ Sinusoid } & \multirow{2}{*}{90} & $50 \%$ \\
\hline OHR2nd-L1-9 & & & & $75 \%$ \\
\hline OHR2nd-L2-8 & \multirow{2}{*}{ L2 } & \multirow{2}{*}{ Sinusoid } & \multirow{2}{*}{90} & $50 \%$ \\
\hline OHR2nd-L2-9 & & & & $75 \%$ \\
\hline OHR2nd-L3-8 & \multirow{2}{*}{ L3 } & \multirow{2}{*}{ Sinusoid } & \multirow{2}{*}{90} & $50 \%$ \\
\hline OHR2nd-L3-9 & & & & $25 \%$ \\
\hline OHR2nd-L4-8 & \multirow{2}{*}{ L4 } & \multirow{2}{*}{ Sinusoid } & \multirow{2}{*}{90} & $50 \%$ \\
\hline OHR2nd-L4-9 & & & & $25 \%$ \\
\hline
\end{tabular}


The WPS for the $90 \mathrm{~Hz}$ sinusoid excitation was already estimated in Section 3.5.1.2, as shown in Table 3.14. In sum, the WPS is between $44-49 \mathrm{~m} / \mathrm{s}$ in the X-direction and between $72-77 \mathrm{~m} / \mathrm{s}$ in the Y-direction. The average WPS is given in Table 3.33.

Table 3.33: Average WPS in X- and Y-Directions for the $90 \mathrm{~Hz}$ Sinusoid Excitation

\begin{tabular}{|c|c|c|}
\cline { 2 - 3 } \multicolumn{1}{c|}{} & $\begin{array}{c}\text { Speed31 (Shaker Power 50\%) } \\
(\mathbf{m} / \mathbf{s})\end{array}$ & $\begin{array}{c}\text { Speed32 (Shaker Power 25\%) } \\
(\mathbf{m} / \mathbf{s})\end{array}$ \\
\hline Y-Dir & 74.5 & 74.4 \\
\hline X-Dir & 47.3 & 47.3 \\
\hline
\end{tabular}

Figure 3.56 shows the effect of various decaying powers on the SRP plots when the excitation is $90 \mathrm{~Hz}$ sinusoid.

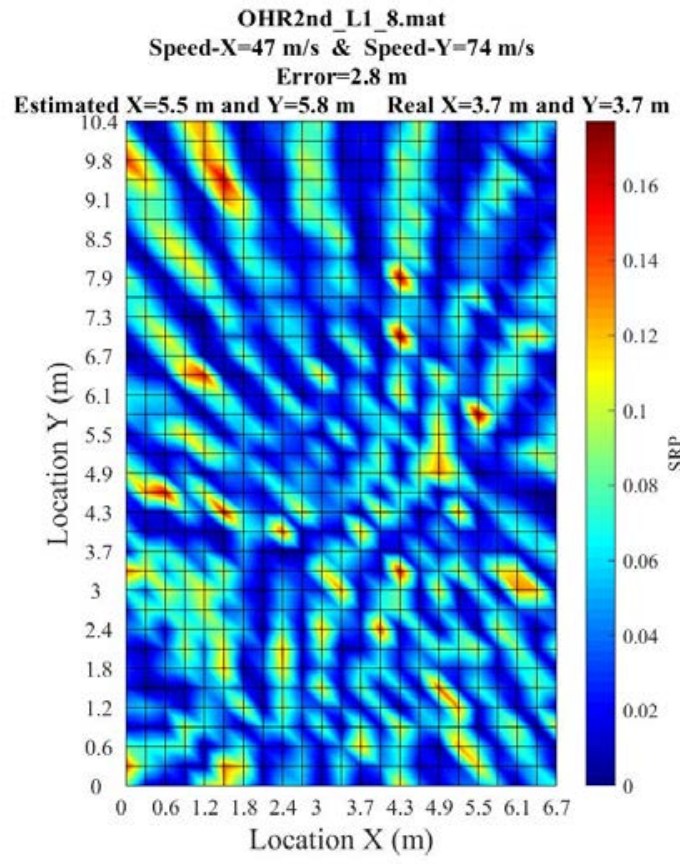

(a) $b=0$

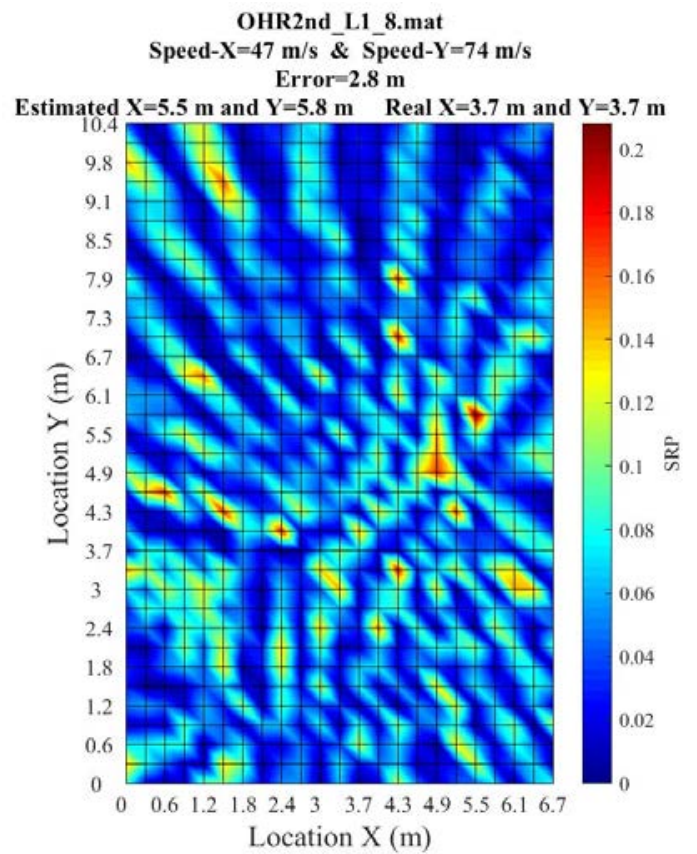

(b) $b=0.2$ 


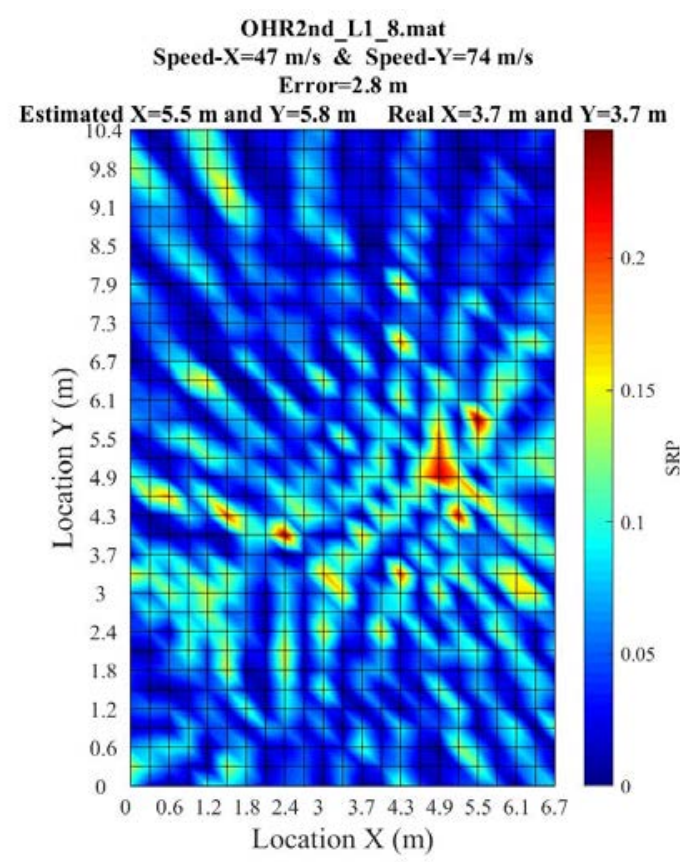

(c) $b=0.4$

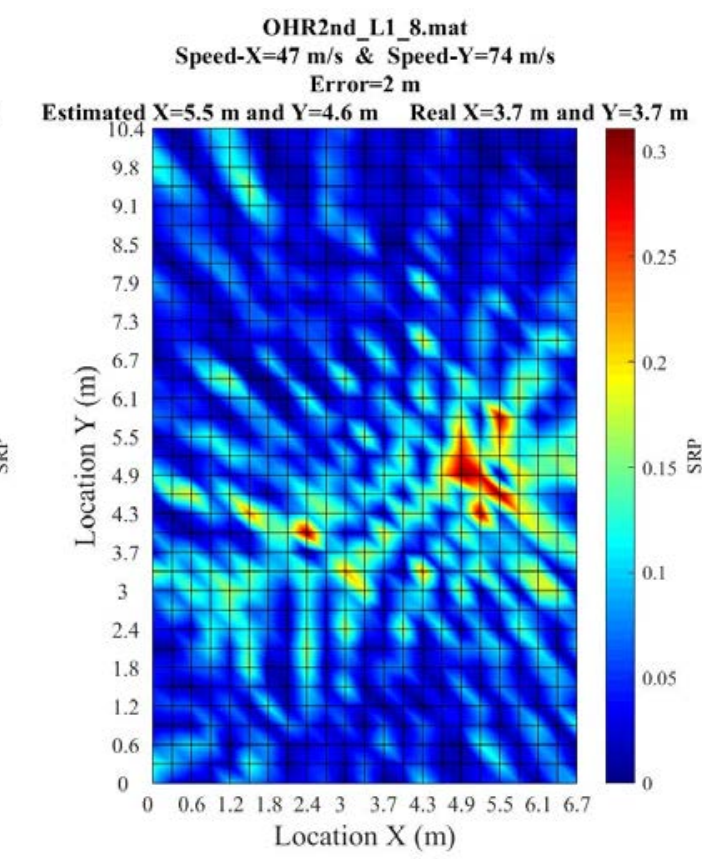

(d) $b=0.6$

Figure 3.56: Effect of Inverse Distance Weighting on the SRP Plots When Considering a Two-Second Waveform from 15-17 sec

For this case, a decaying power of 0.4 seems suitable. This decaying power is stated in the captions of Figures 3.57 and 3.58 below. Also, note that, in this section, a filter with a frequency range of $80 \mathrm{~Hz}$ to $100 \mathrm{~Hz}$ was used. 


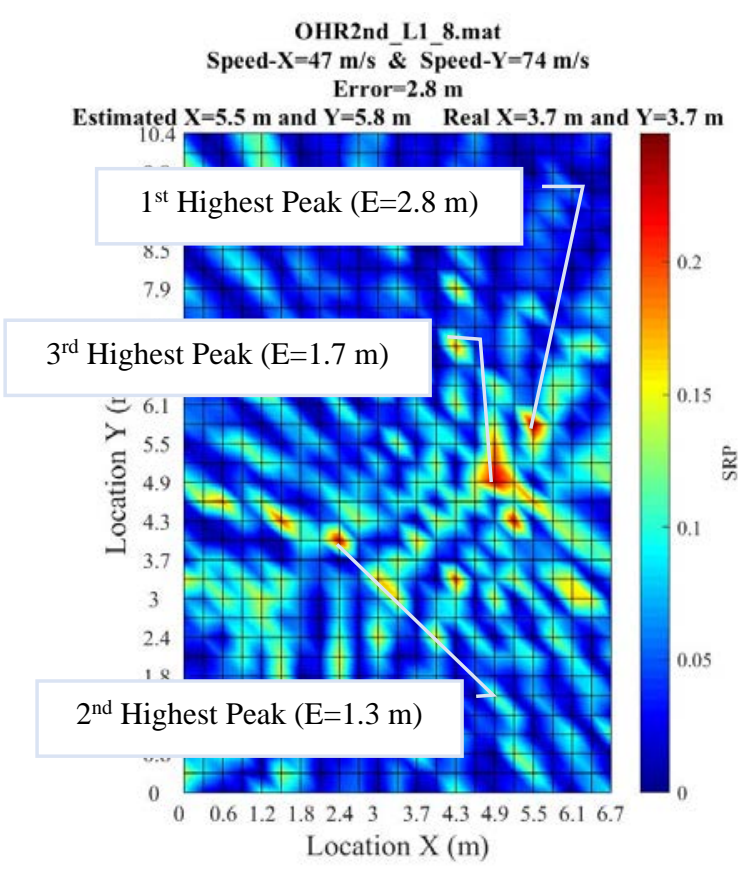

(a) Test OHR2nd-L1-8

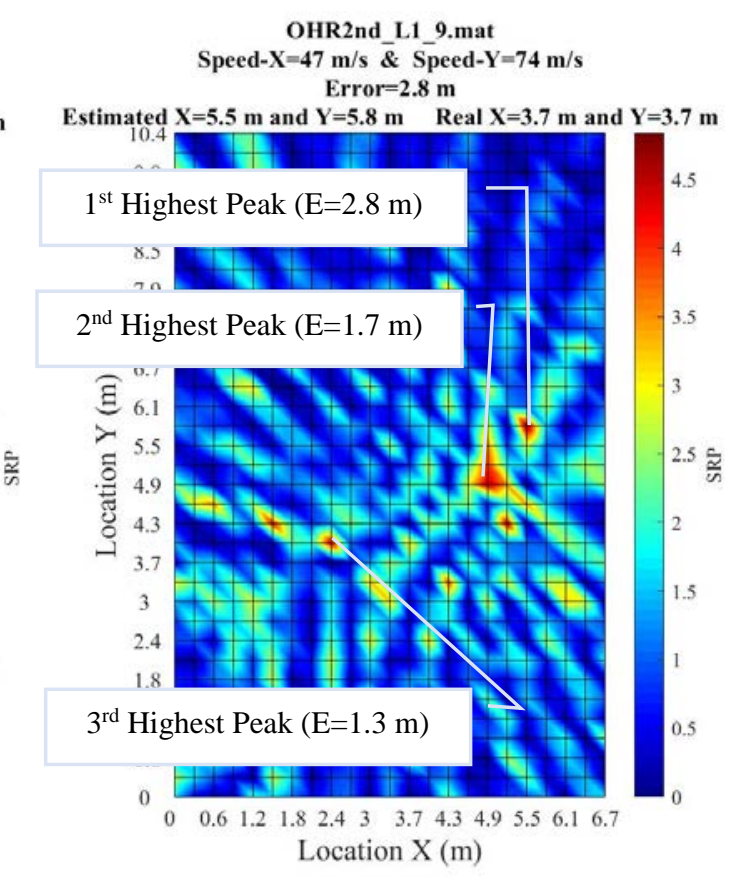

(b) Test OHR2nd-L1-9

Figure 3.57: SRP Plots When Considering a Two-Second Waveform from 15-17 sec and Filtering in a Frequency Range of $80-100 \mathrm{~Hz}(b=0.4)$

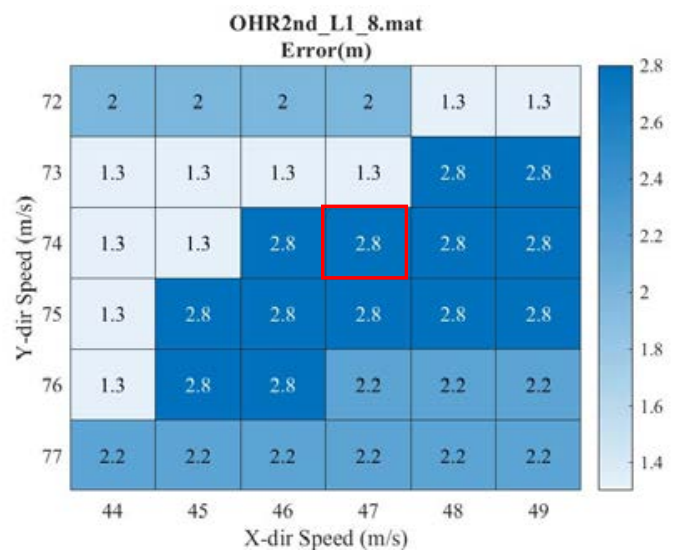

(a) Test OHR2nd-L1-8

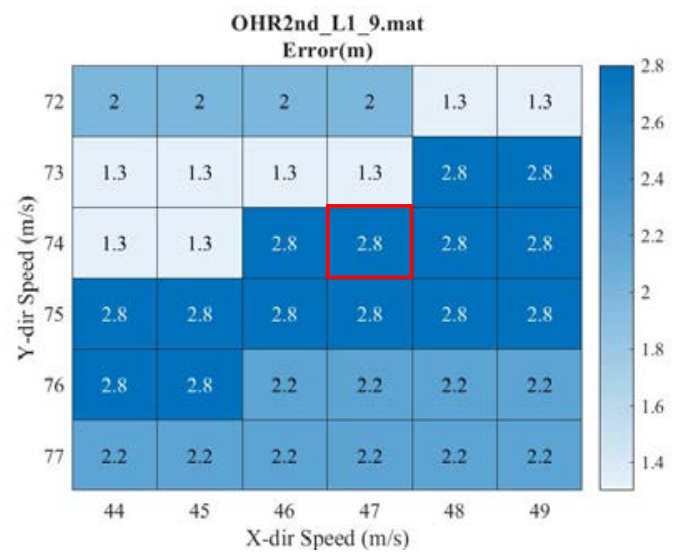

(b) Test OHR2nd-L1-9

Figure 3.58: Localization Error Plots When Considering a Two-Second Waveform from 15-17 sec and Filtering in a Frequency Range of $80-100 \mathrm{~Hz}(b=0.4)$

Although the same decaying power is used in Figures 3.57(a) and 3.57(b), the orders of the peaks are different. The reason for this is that the shaker power was very high in test OHR2nd-L1-9 (i.e., more than 0.3g), and, as a result, the response of the A2 
accelerometer was relatively higher than the other accelerometers (see Figure 3.59). This is another reason why it is helpful to see the error associated with the first three highest peaks.
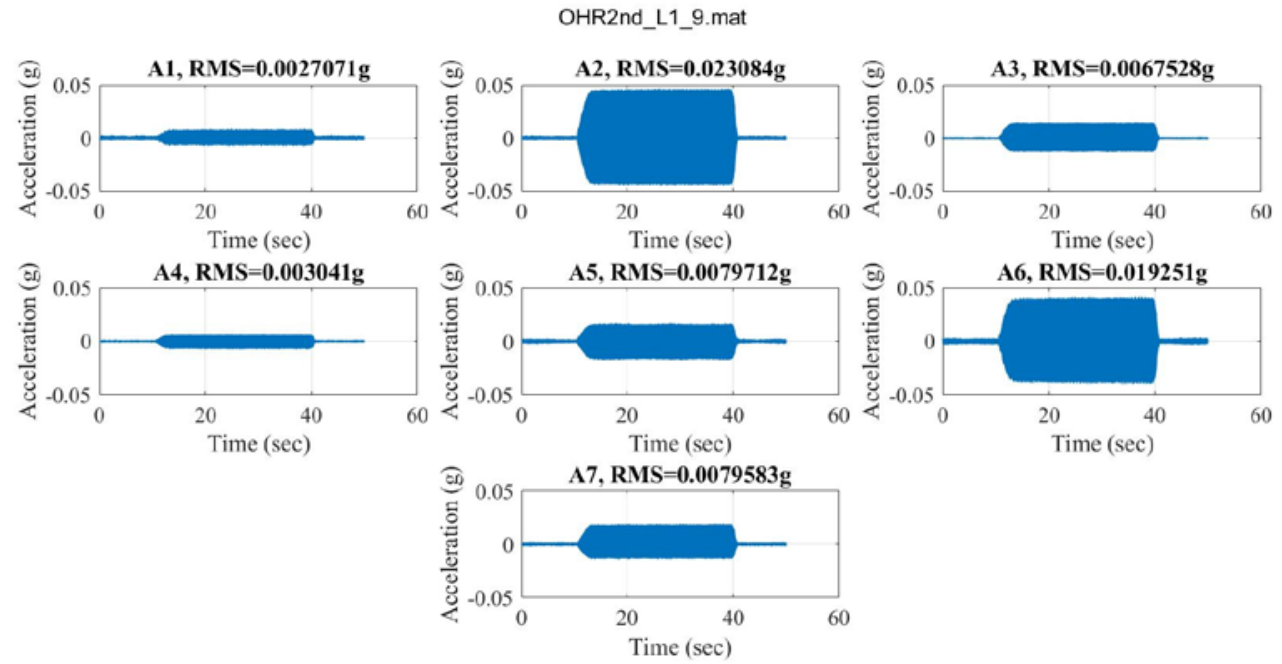

Figure 3.59: Response to the $90 \mathrm{~Hz}$ Sinusoid Excitation

The SRP plots and corresponding localization errors for the rest of the tests (listed in Table 3.32) when the shaker was placed at L2, L3, and L4 are shown in Appendix A.3.

Table 3.34 shows the localization errors associated with the first, second, and third highest peaks when the WPS in the X- and Y-directions is $47 \mathrm{~m} / \mathrm{s}$ and $74 \mathrm{~m} / \mathrm{s}$. The minimum error for each test is highlighted in green.

Table 3.34: Localization Errors for the First, Second, and Third Highest Peaks When the WPS in X- and Y-Directions is $47 \mathrm{~m} / \mathrm{s}$ and $74 \mathrm{~m} / \mathrm{s}$

\begin{tabular}{|c|c|c|c|c|}
\hline \multirow{2}{*}{ Test Name } & \multirow{2}{*}{$\begin{array}{c}\text { Sinusoid } \\
\text { Excitation }\end{array}$} & \multicolumn{3}{|c|}{ Error Corresponding to Peaks } \\
\cline { 3 - 5 } & & $\mathbf{1}^{\text {st }}$ Highest & $\mathbf{2}^{\text {nd }}$ Highest & $3^{\text {rd }}$ Highest \\
\cline { 3 - 5 } & & $\mathbf{( m )}$ & $\mathbf{( m )}$ & $\mathbf{( m )}$ \\
\hline OHR2nd_L1_8 & $90 \mathrm{~Hz}$ & 2.8 & 1.3 & 1.7 \\
\hline OHR2nd_L1_9 & $90 \mathrm{~Hz}$ & 2.8 & 1.7 & 1.3 \\
\hline OHR2nd_L2_8 & $90 \mathrm{~Hz}$ & 1.9 & 2.4 & 5.8 \\
\hline OHR2nd_L2_9 & $90 \mathrm{~Hz}$ & 1.9 & 2.4 & 5.8 \\
\hline OHR2nd_L3_8 & $90 \mathrm{~Hz}$ & 2.6 & 1.7 & 5.9 \\
\hline OHR2nd_L3_9 & $90 \mathrm{~Hz}$ & 2.6 & 1.7 & 5.9 \\
\hline OHR2nd_L4_8 & $90 \mathrm{~Hz}$ & 3.2 & 3.4 & 2.9 \\
\hline OHR2nd_L4_9 & $90 \mathrm{~Hz}$ & 3.2 & 6.5 & 3.4 \\
\hline
\end{tabular}




\subsubsection{110 Hz Sinusoid Excitation}

The tests for the $110 \mathrm{~Hz}$ sinusoid excitation are shown in Table 3.35 .

Table 3.35: List of Tests with $110 \mathrm{~Hz}$ Sinusoid Excitation

\begin{tabular}{|c|c|c|c|c|}
\hline Test Name & \begin{tabular}{|c|} 
Shaker \\
Location
\end{tabular} & Excitation & $\begin{array}{c}\text { Frequency Range } \\
\mathrm{Hz}\end{array}$ & $\begin{array}{l}\text { Shaker } \\
\text { Power }\end{array}$ \\
\hline OHR2nd-L1-10 & \multirow{2}{*}{ L1 } & \multirow{2}{*}{ Sinusoid } & \multirow{2}{*}{110} & $50 \%$ \\
\hline OHR2nd-L1-11 & & & & $37.5 \%$ \\
\hline OHR2nd-L2-10 & \multirow{2}{*}{ L2 } & \multirow{2}{*}{ Sinusoid } & \multirow{2}{*}{110} & $50 \%$ \\
\hline OHR2nd-L2-11 & & & & $37.5 \%$ \\
\hline OHR2nd-L3-10 & \multirow{2}{*}{ L3 } & \multirow{2}{*}{ Sinusoid } & \multirow{2}{*}{110} & $50 \%$ \\
\hline OHR2nd-L3-11 & & & & $25 \%$ \\
\hline OHR2nd-L4-10 & \multirow{2}{*}{ L4 } & \multirow{2}{*}{ Sinusoid } & \multirow{2}{*}{110} & $50 \%$ \\
\hline OHR2nd-L4-11 & & & & $25 \%$ \\
\hline
\end{tabular}

The WPS was already estimated in Section 3.5.1.2 for $110 \mathrm{~Hz}$ sinusoid excitation, as shown in Table 3.15. In sum, the WPS is between $46-50 \mathrm{~m} / \mathrm{s}$ in the X-direction and between 102-109 m/s in the Y-direction. The average WPS is given in Table 3.36.

Table 3.36: Average WPS in X-and Y-Directions for $110 \mathrm{~Hz}$ Sinusoid Excitation

\begin{tabular}{|c|c|c|}
\cline { 2 - 3 } \multicolumn{1}{c|}{} & $\begin{array}{c}\text { Speed33 (Shaker Power 37.5\%) } \\
(\mathbf{m} / \mathbf{s})\end{array}$ & $\begin{array}{c}\text { Speed34 (Shaker Power 50\%) } \\
(\mathbf{m} / \mathbf{s})\end{array}$ \\
\hline Y-Dir & 106.0 & 104.6 \\
\hline X-Dir & 48.1 & 47.5 \\
\hline
\end{tabular}

Figure 3.60 shows the SRP plots for the tests in which the shaker was placed at L1. In this section, the filter was used in a frequency range of $100 \mathrm{~Hz}$ to $120 \mathrm{~Hz}$. The localization errors for the highest steered power is shown in Figure 3.61. 


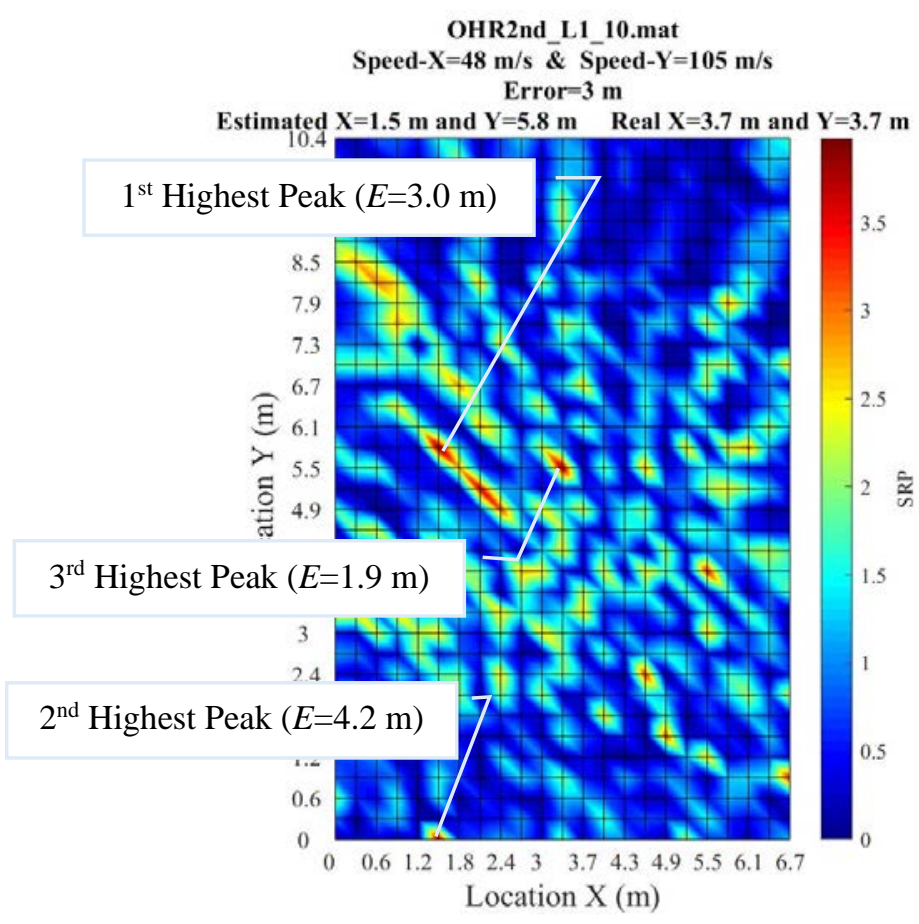

(a) Test OHR2nd_L1_10

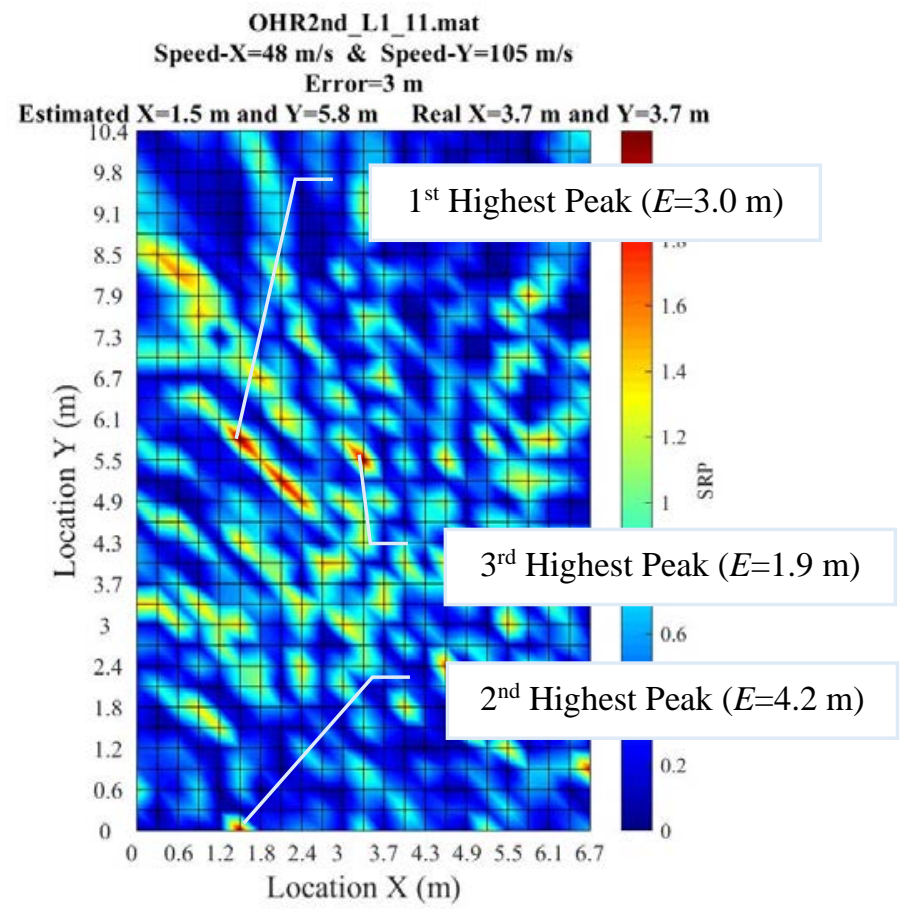

(b) Test OHR2nd_L1_11

Figure 3.60: SRP Plots When Considering a Two-Second Waveform from 15-17 sec and Filtering in a Frequency Range of $100-120 \mathrm{~Hz}(b=0.5)$

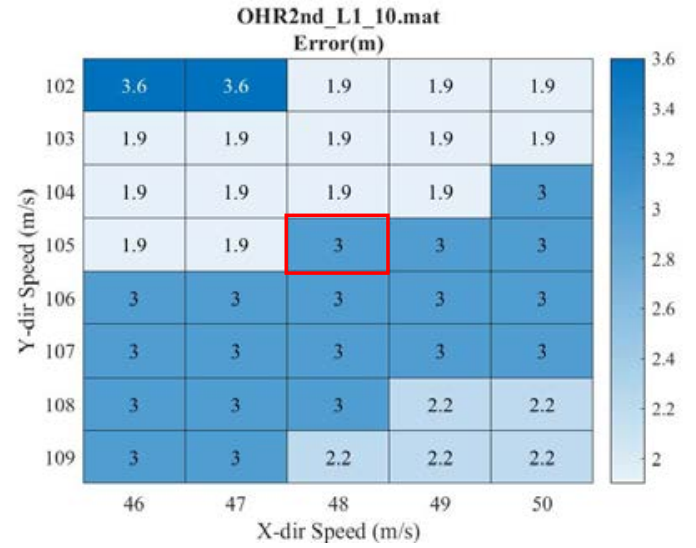

(a) Test OHR2nd_L1_10

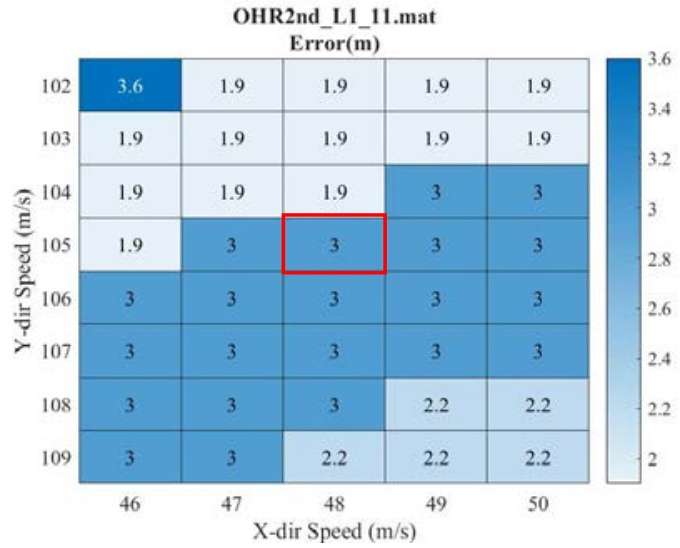

(b) Test OHR2nd_L1_11

Figure 3.61: Localization Error Plots When Considering a Two-Second Waveform from $15-17$ sec and Filtering in a Frequency Range of $100-120 \mathrm{~Hz}(b=0.5)$

The SRP and localization error plots for the rest of the tests (listed in Table 3.35) in which the shaker was placed at L2, L3, and L4 are shown in Appendix A.4. 
Table 3.37 shows the errors associated with the three highest peaks when the WPS in the $\mathrm{X}$ - and $\mathrm{Y}$-directions is $48 \mathrm{~m} / \mathrm{s}$ and $105 \mathrm{~m} / \mathrm{s}$. The minimum error for each test is highlighted in green.

Table 3.37: Localization Errors for the First, Second, and Third Highest Peaks When the WPS in X- and Y-Directions is $48 \mathrm{~m} / \mathrm{s}$ and $105 \mathrm{~m} / \mathrm{s}$

\begin{tabular}{|c|c|c|c|c|}
\hline \multirow{2}{*}{ Test Name } & \multirow{2}{*}{$\begin{array}{c}\text { Sinusoid } \\
\text { Excitation }\end{array}$} & \multicolumn{3}{|c|}{ Error Corresponding to Peaks } \\
\cline { 3 - 5 } & & 1st Highest & 2nd Highest & 3rd Highest \\
\cline { 3 - 5 } & $\mathbf{( m )}$ & $\mathbf{( m )}$ & $\mathbf{( m )}$ \\
\hline OHR2nd_L1_10 & $110 \mathrm{~Hz}$ & 3.0 & 4.2 & 1.9 \\
\hline OHR2nd_L1_11 & $110 \mathrm{~Hz}$ & 3.0 & 4.2 & 1.9 \\
\hline OHR2nd_L2_10 & $110 \mathrm{~Hz}$ & 2.0 & 3.0 & 2.3 \\
\hline OHR2nd_L2_11 & $110 \mathrm{~Hz}$ & 2.0 & 3.0 & 2.3 \\
\hline OHR2nd_L3_10 & $110 \mathrm{~Hz}$ & 2.0 & 2.7 & 2.5 \\
\hline OHR2nd_L3_11 & $110 \mathrm{~Hz}$ & 2.0 & 2.7 & 2.5 \\
\hline OHR2nd_L4_10 & $110 \mathrm{~Hz}$ & 3.3 & 3.0 & 2.8 \\
\hline OHR2nd_L4_11 & $110 \mathrm{~Hz}$ & 3.3 & 3.0 & 2.8 \\
\hline
\end{tabular}

The results shown in Tables 3.28, 3.31, 3.34, and 3.37 are depicted in Figures 3.62(a), 3.62(b), 3.62(c), and 3.62(d), respectively. The localization error corresponding to the first, second, and third highest steered powers are shown in blue, red, and orange colors, respectively, in these figures. The ticks of X-axes of subfigures in Figure 3.62 represent the list of tests. Note that the area of the tested floor was $13.4 \mathrm{~m}$ by $8.4 \mathrm{~m}$.

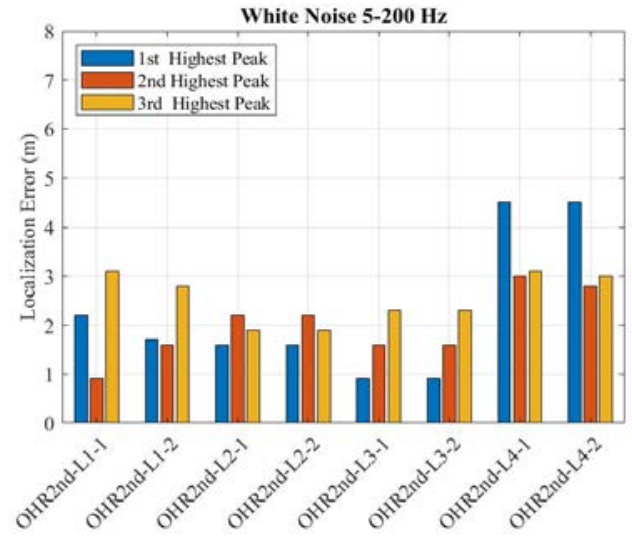

(a) White Noise Excitation

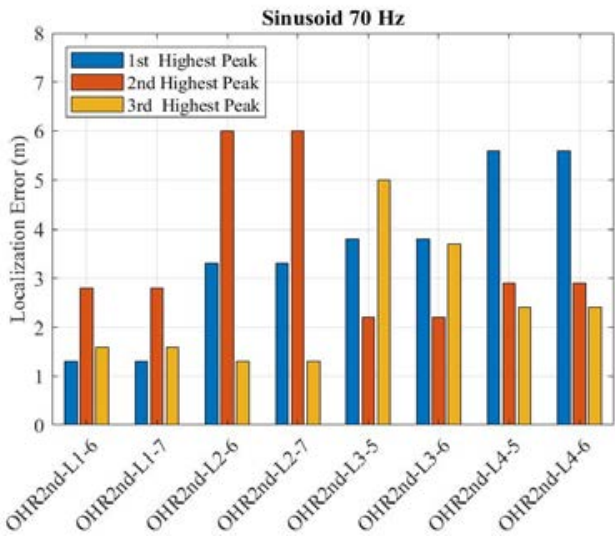

(b) $70 \mathrm{~Hz}$ Sinusoid Excitation 


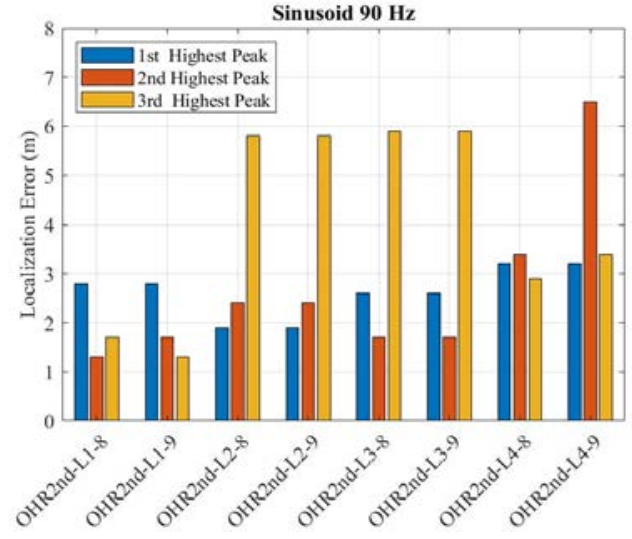

(c) $90 \mathrm{~Hz}$ Sinusoid Excitation

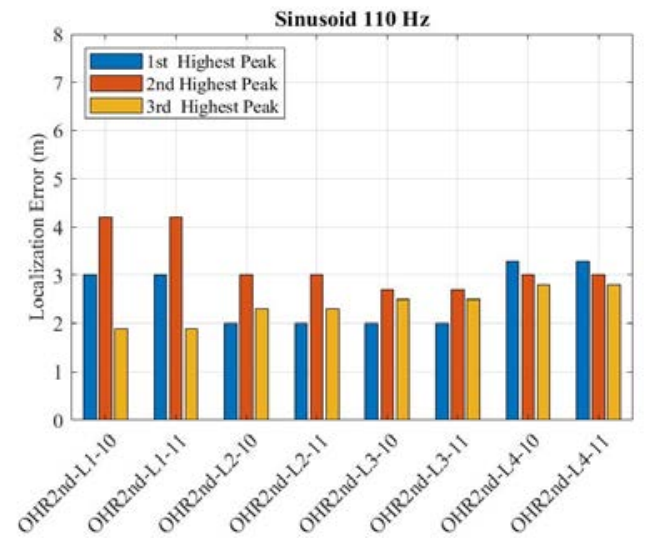

(d) $110 \mathrm{~Hz}$ Sinusoid Excitation

Figure 3.62: Localization Errors 


\section{CHAPTER 4: SUMMARY, CONCLUSION, AND RECOMMENDATION FOR FUTURE RESEARCH}

\subsection{Summary}

This study aims to introduce a practical approach for locating the vibration source on a concrete floor system. Any vibration problem consists of three factors: source, path, and receiver. This study focuses on stationary sources of vibration, such as chillers, fans, HVAC units, and water pumps, and the path is the concrete floor system. The problem occurs when a source applies a dynamic load on the concrete floor, causing the floor to vibrate, and the generated vibration exceeds the acceptable limits of the receiver. These limits are associated with human comfort (e.g., surgery room), animals for research (e.g., vivarium room), or sensitive instrument items (e.g., CT scanners and high-power microscopes). Therefore, the vibration source needs to be identified, which is a process known as localization. Toward that end, the Steered Response Power (SRP) method was proposed. This method has already been successfully used for electromagnetic and acoustical applications to locate the transmitter and sound sources, respectively. In such applications, the input data is either wireless or audio signals. However, for the application presented in this study, the input data is acceleration signals acquired by various accelerometers placed on the concrete floor. Therefore, the SRP method needed to be modified to be applicable in this study.

In the SRP approach, the regions of potential source locations are rated by applying the propagation delays from each point in the region to the ensemble of signals received at each sensor. The steered power associated with each candidate location is then computed, and the location with the highest power is the most likely source. If a source is at that location, the time signals will align when summed across the ensemble, and a local maximum of power would result. If no source is at the location, the alignment will have destructive elements when summed and sum to a lesser value. The final localization 
decision can only be made when the computation of steered power for all candidate source locations has been computed.

It was discussed in Chapter 1 that the effects of multipath propagation, attenuation, and dispersion make the vibration-sensing-localization approaches a challenging task. Furthermore, some localization approaches were introduced, and their performances for the present study were examined. The Received Signal Strength (RSS) method requires a pre-defined attenuation pattern, which is unknown for a concrete floor. It is also assumed in this approach that the energy of propagated vibration decreases as the distance between source and sensor increases, and thus, a sensor farther from the source is expected to measure a lower amplitude than a sensor closer to it. However, this assumption may not be valid for a concrete floor. Time Difference of Arrival (TDOA) is another localization approach based on ideal vibration propagation (e.g., constant WPS in all directions), which is not the case with many real-world structural systems, such as concrete floors. In contrast, regarding Equation 3.11, the SRP method can, more easily, account for different propagation speeds in multiple directions. Although the SRP method is computationally more demanding than TDOA-based localization, it has been proven to be more robust in some applications (DiBiase et al. 2001).

The concepts and equations of the SRP method were introduced in Chapter 2, and their applications for different sets of experiments in a full-scale computer lab were discussed in Chapter 3. This lab was located on the second floor in the Oliver H. Raymond (OHR) building at the University of Kentucky campus. The estimation of wave propagation speed (WPS) was discussed in Section 3.5. In this section, two approaches were also proposed for estimating WPS: the time-domain approach and the frequencydomain approach. This study showed the superior performance of the time-domain implementation in processing acceleration data acquired from a concrete floor. Variations in the estimated time delays in the time-domain approach were less than $1.0 \mathrm{~m} / \mathrm{s}$ in different cases shown in Figure 3.17. However, the lack of robustness in the frequencydomain approach was shown by the fact that the variation among the different cases was considerably high. Table 4.1 shows the range of WPS estimates in two orthogonal directions. X- and Y-directions were shown in Figure 3.37. 
Table 4.1: Range of WPS Estimates

\begin{tabular}{|c|c|c|c|c|}
\cline { 2 - 5 } \multicolumn{1}{c|}{} & \multicolumn{4}{c|}{ Range of Wave Propagation Speed (WPS) Estimates (m/s) } \\
\cline { 2 - 5 } \multicolumn{1}{c|}{} & $\begin{array}{c}\text { White Noise } \\
\text { Excitation }\end{array}$ & $\begin{array}{c}\mathbf{7 0} \text { Hz Sinusoid } \\
\text { Excitation }\end{array}$ & $\begin{array}{c}\mathbf{9 0} \text { Hz Sinusoid } \\
\text { Excitation }\end{array}$ & $\begin{array}{c}\mathbf{1 1 0} \text { Hz Sinusoid } \\
\text { Excitation }\end{array}$ \\
\hline X-Direction & $72-80$ & $35-39$ & $44-49$ & $46-50$ \\
\hline Y-Direction & $102-113$ & $59-62$ & $72-77$ & $102-109$ \\
\hline
\end{tabular}

To assess the performance of the SRP method, four types of excitation forces were applied by the shaker: white noise, $70 \mathrm{~Hz}$ sinusoid, $90 \mathrm{~Hz}$ sinusoid, and $110 \mathrm{~Hz}$ sinusoid (see Section 3.6). For each excitation force, the shaker was placed at L1, L2, L3, and L4 locations, in that order (see Figure 3.37). At each location, excitation force was applied twice with two shaker powers. Therefore, eight tests were conducted for each type of excitation force. The results were shown in Figure 3.62.

The SRP plot can only be generated for a specific input propagation speed in Xand Y-directions. However, the estimated WPS was achieved in a range of values mentioned in Table 4.1. Thus, various input propagation speeds resulted in different maximum steered powers. Therefore, the three highest steered powers were selected, and the localization error (i.e., the distance between estimated and actual vibration source) associated with each of them was recorded. The minimum localization error is the lowest error among the first, second, and third highest peaks (shown in Figure 4.1), which is depicted in Figure 4.1 for each type of excitation force. The $\mathrm{X}$-axis shows the type of excitation force, and the test names are stated inside each bar plot. Note that the area of the tested floor was approximately $13.4 \mathrm{~m}$ by $8.4 \mathrm{~m}$. 


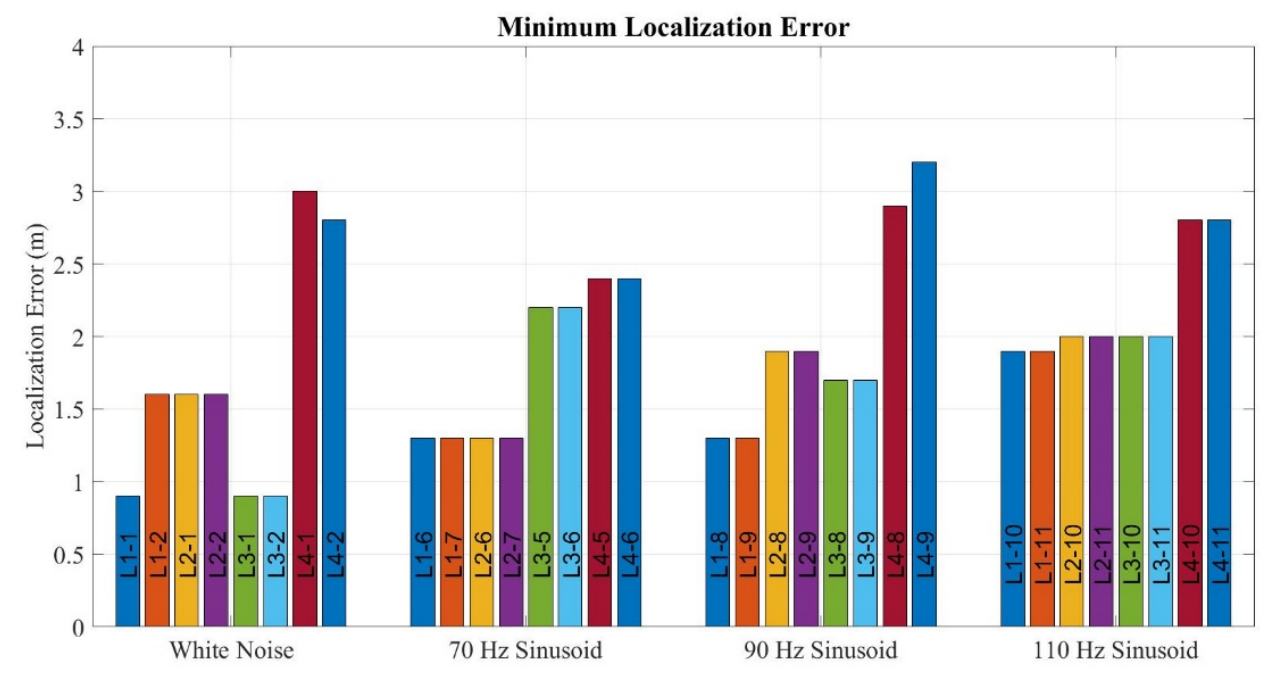

Figure 4.1: Minimum Localization Error

The second column in Table 4.2 shows the average localization error associated with Figure 4.1 for each type of excitation force. The fourth column in this table shows the average localization error regardless of tests when the shaker was placed at L4. The standard deviation is also shown in Table 4.2. The average error for all thirty two tests is $1.91 \mathrm{~m}$, which is less than the acceptable level of accuracy of $3.0 \mathrm{~m}$ defined in Section 1.3.

Table 4.2: Average Error

\begin{tabular}{|c|c|c|c|c|}
\multicolumn{1}{c|}{} & \multicolumn{2}{|c|}{ Shaker at L1, L2, L3, and L4 } & \multicolumn{2}{c|}{ Shaker at L1, L2, and L3 } \\
\hline Excitation Force & $\begin{array}{c}\text { Average Error } \\
\mathbf{( m )}\end{array}$ & $\begin{array}{c}\text { Standard } \\
\text { Deviation }(\mathbf{m})\end{array}$ & $\begin{array}{c}\text { Average Error } \\
\mathbf{( m )}\end{array}$ & $\begin{array}{c}\text { Standard } \\
\text { Deviation (m) }\end{array}$ \\
\hline White Noise & 1.66 & 0.83 & 1.25 & 0.38 \\
\hline $70 \mathrm{~Hz}$ Sinusoid & 1.8 & 0.54 & 1.6 & 0.46 \\
\hline $90 \mathrm{~Hz}$ Sinusoid & 1.98 & 0.7 & 1.63 & 0.27 \\
\hline $110 \mathrm{~Hz}$ Sinusoid & 2.18 & 0.39 & 1.97 & 0.05 \\
\hline Total & 1.91 & 0.64 & 1.61 & 0.4 \\
\hline
\end{tabular}

As shown in Figure 4.1 and Table 4.2, the localization errors corresponding to the tests with the shaker at L4 are higher than the rest of the tests. This may be because the distance between the shaker at L4 and the masonry wall and concrete girder (girder size is approximately $0.6 \mathrm{~m}$ by $0.6 \mathrm{~m}$ ) located on column line E (Figure 3.6) was approximately $1.0 \mathrm{~m}$. Generally, it is expected that the areas near the walls and, especially, the girders to 
be stiffer. Therefore, the responses received by the accelerometers were weaker than in tests where the shaker was placed at L1, L2, and L3. For example, Figure 4.2 shows the excitation force and received signals for test $O H R 2 n d \_L 4 \_2$ in which the shaker was placed at L4. Although the shaker generated an excitation force of up to approximately 40 $\mathrm{N}$, the signal received by the A3 accelerometer did not exceed 0.005g. Despite being located closest to the shaker at a distance of $1.4 \mathrm{~m}$, the A3 accelerometer nevertheless received the lowest power signal of all accelerometers. Compare Figure 4.2(b) with Figure 4.3 when the shaker was placed at L1 location. The excitation force in both cases was almost the same.

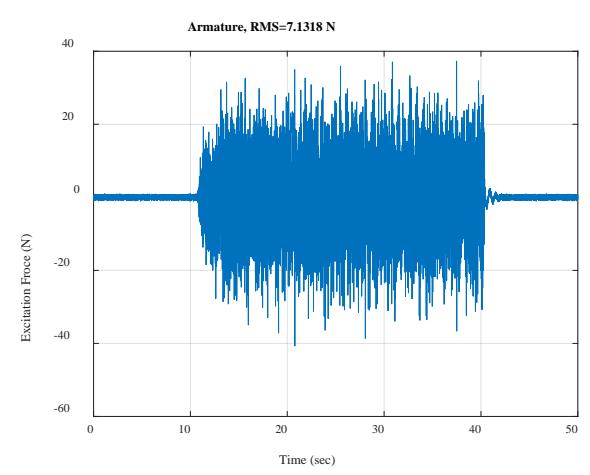

(a) Applied White Noise Excitation Force

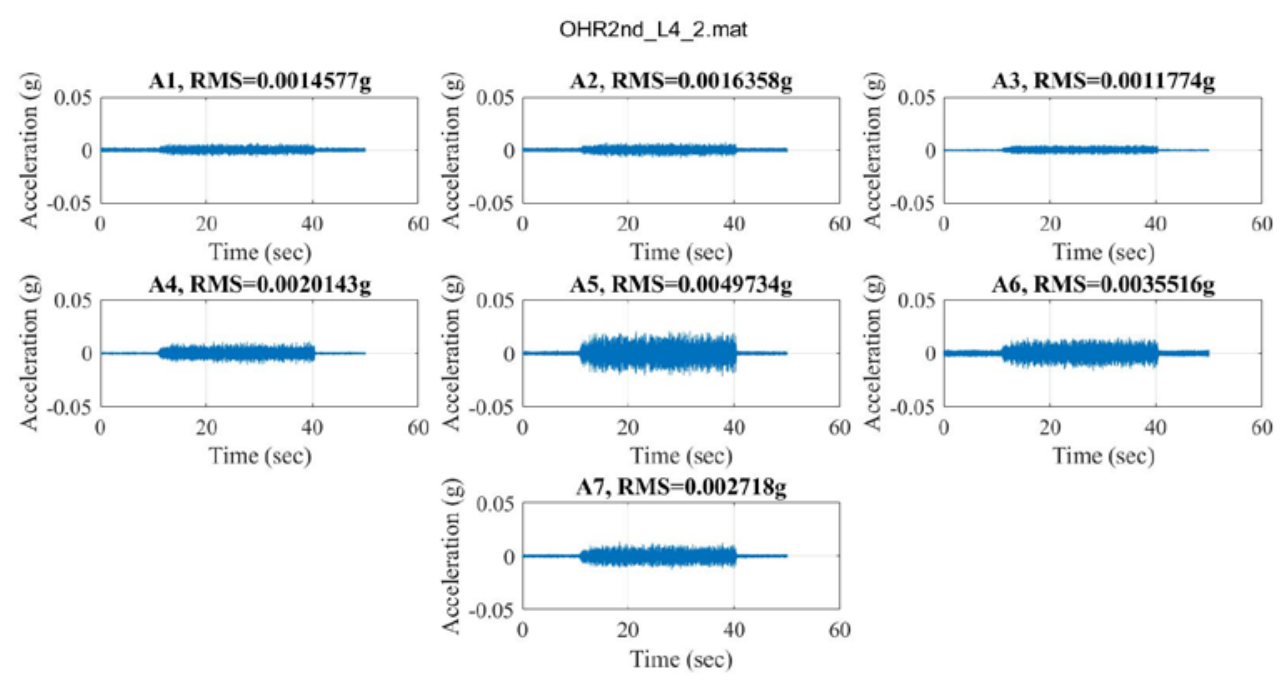

(b) Received Acceleration Data due to White Noise Excitation Force

Figure 4.2: Excitation and Received Accelerations for Test OHR2nd-L4-2 (Shaker at L4) 

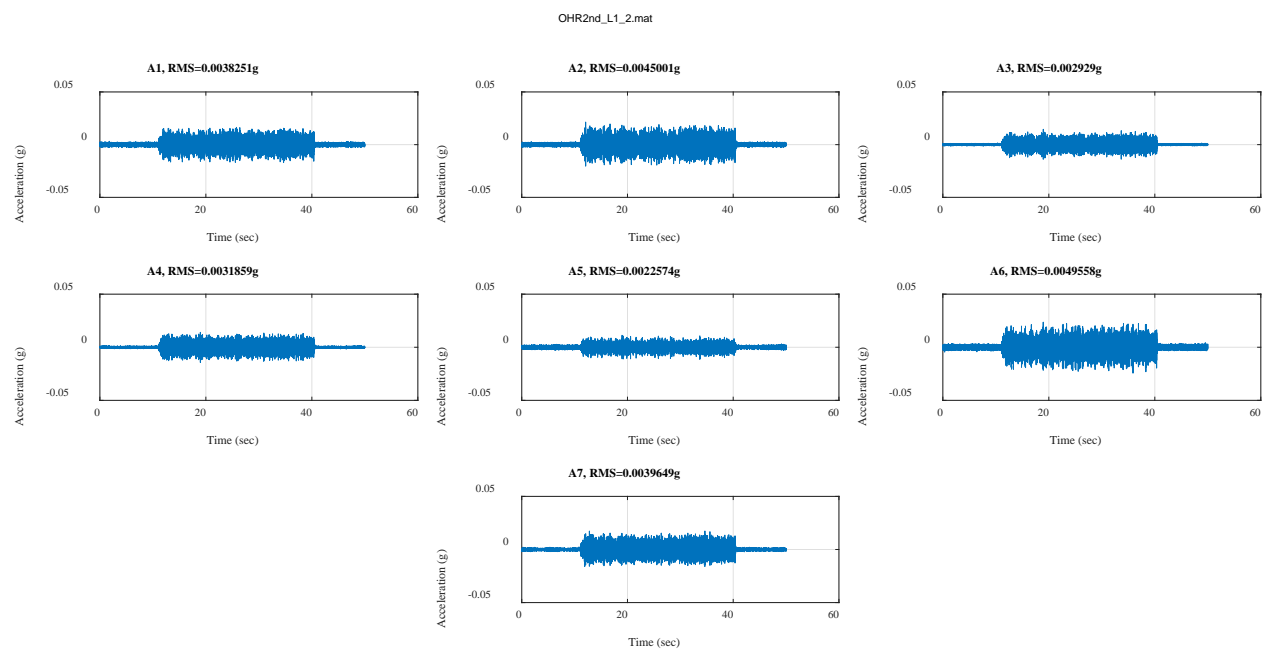

Figure 4.3: Excitation and Received Accelerations for Test OHR2nd-L1-2 (Shaker at L1)

It was discussed in Section 2.2 (Simulations 9 and 10) that the injudicious placement of accelerometers can cause ambiguity in the SRP plot. The L2 location was placed very close to the four bisectors between A2 and A3, A3 and A5, A5 and A6, and A6 and A2 (this is shown by red arrows in Figure 4.4). Nevertheless, the localization errors are almost the same as in L1, L3, and L4. This advantage comes from using a large number of accelerometers. 


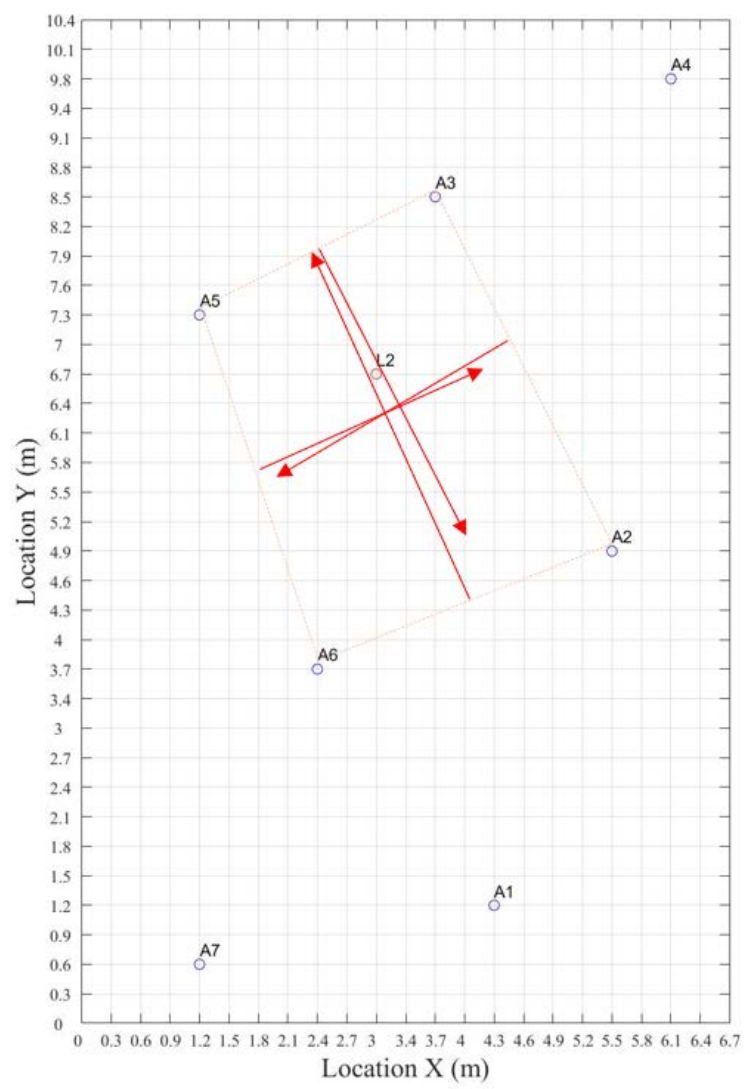

Figure 4.4: L2 Location Versus Bisectors Between the Accelerometers

For the test series with $70 \mathrm{~Hz}$ sinusoid excitation, the localization errors for the tests where the shaker was placed at L2, L3, and L4 were also high. The reason for this may be due to the fact that the tested floor was barely responsive to the $70 \mathrm{~Hz}$ vibration excitation, as shown by the FRF plots in Figure 3.11. Hence, the maximum vibration accelerations in these tests barely exceeded $0.02 \mathrm{~g}$, even when the shaker worked with $75 \%$ of its power and was applying more than $78 \mathrm{~N}$ force on the floor. In other words, based on the FRF plots of the tested floor, it is unlikely that a $70 \mathrm{~Hz}$ sinusoid vibration excitation will cause any issue. Figures 4.5(a) and 4.5(b) show the excitation accelerometer and received responses for test OHR2nd-L2-7. 


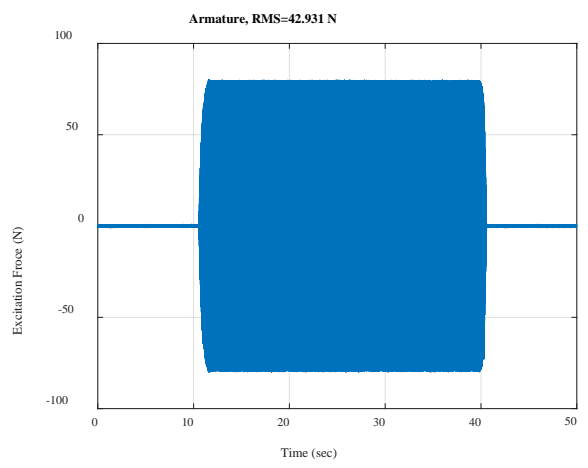

(a) Applied $70 \mathrm{~Hz}$ Sinusoid Excitation Force
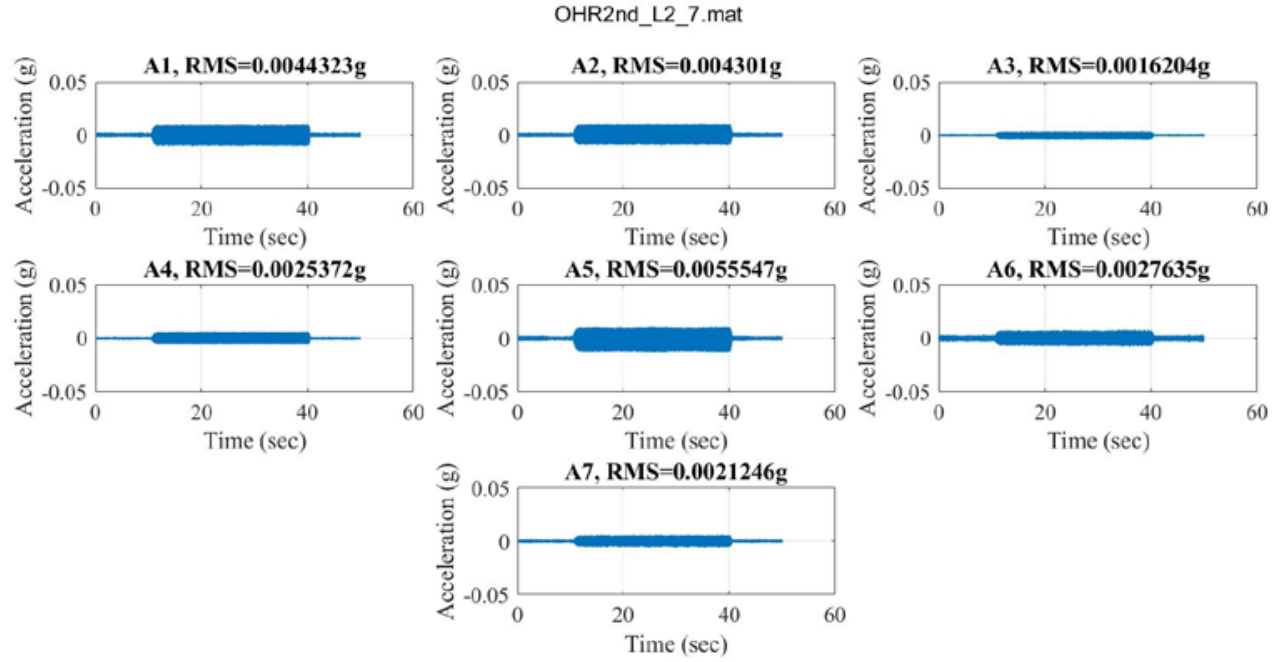

(b) Received Acceleration Data due to $70 \mathrm{~Hz}$ Sinusoid Excitation Force

Figure 4.5: Applied Force Versus Received Responses for Test OHR2nd-L2-7

\subsection{Conclusions}

Given what has been demonstrated, the following conclusions can be made:

- As shown in Figure 4.1 and Table 4.2, the average localization error using the SRP method is $1.91 \mathrm{~m}$ on a floor of approximately $13.4 \mathrm{~m}$ by $8.4 \mathrm{~m}$ size. As discussed in Section 4.1, by excluding the tests where the shaker was located at L4, the average localization error can drop to $1.61 \mathrm{~m}$.

- It has been explained in Sections 1.2.3 and 2.1 that the SRP method is suitable for near-field vibration localization, such as in indoor building environments. 
- The SRP method is more robust in the presence of structural variability - i.e., the non-uniform distribution of material properties and various boundary conditions in different bays - and attenuation, dispersion, and multipath effects, which are the main challenges in other indoor vibration-sensing localization approaches. Although theoretically, this algorithm can also be employed in other vibrationsensing localization applications, this dissertation is limited to the capability and sufficiency of this method to locate machinery-induced vibration (see Section 1.3).

- The key parameter that is unknown and required in this localizer method is wave propagation speed (WPS), which can be estimated using empirical tests. The precision of the SRP method relies on the accurate estimation of WPS. For the estimation of the WPS, the time-domain approach outperformed the frequencydomain approach, as discussed in Sections 2.3 and 3.5.

- A constant WPS in all directions is not a realistic assumption and decreases the accuracy of the SRP method for the application presented herein. More specifically, because the geometry, boundary conditions, and stiffness of a concrete floor typically vary in orthogonal directions, it makes sense to assume different propagation speeds when using this method. The propagation speeds in the orthogonal directions are used in the SRP method, and the method is capable of automatically interpolating the corresponding propagation speed for any of these directions (see Equation 3.11). This is one of the advantages of the SRP method over the TDOA.

- As discussed in Section 2.1, the SRP method correctly locates the vibration source if the source and sensors are placed on a single path, e.g., the same elevated concrete floor. However, if the waves from the vibration source travel through multiple paths (e.g., through the upper floor slab to a column and through the column to the lower floor slab) to the sensors, the SRP method will not correctly locate the source of vibration. In such a case, the SRP method misidentifies the column as the source from which the vibration waves are transmitted.

- With the use of different simulations described in Section 2.2, it has been explained that how grid resolution, sampling frequency, noise, and judicious placement of 
sensors can increase the accuracy of the SRP method and reduce the ambiguity of plots.

- As discussed in Section 3.6, the use of inverse distance weighting (Equation 2.12) can significantly improve the accuracy of results in the presence of attenuation.

\subsection{Recommendations for Future Research}

Based on the findings in this dissertation, the following recommendations can be suggested:

- This study was focused on locating a vibration source when both the source and accelerometers are placed on a single bay. The second phase of the study can be evaluating the performance of the SRP method when both the source and sensors are placed on multiple bays at the same elevated floor.

- It was explained in Section 2.1 that the SRP method could correctly locate the vibration source if both the source and all the accelerometers are placed on a single path. In the case of multiple paths, the SRP method will not be able to locate the source (Yu and Donohue 2013). However, in such a case, the SRP plot provides a clue regarding which part of the concrete floor the vibration is coming from (e.g., a column). Therefore, the source can be either on the lower or upper floor. This opens the gate for the third phase of the study to locate the source among different elevated floors in a single building.

- This research focuses on stationary vibration source. The SRP method might be capable of locating moving vibration sources (e.g., a person walking) using realtime data-processing (see Dmochowski et al. 2007). Toward that end, the computation should be more efficient.

- The precision of the SRP method depends on the accuracy of the estimated WPS. In this study, a set of tests was used to empirically estimate the WPS in two orthogonal directions, a process that requires the use of a shaker. As already explained, using a shaker is costly and time-consuming. A theoretical approach to estimating WPS, such as the use of a finite element model, may make this process both cheaper and faster. 
- The other motivation behind the use of a shaker in this study was to generate controlled vibration with a specific frequency to suppress the effect of dispersion. Although hammer tests or even heel-drops are much faster and more economical, these would generate a wide frequency range, and the dispersion might affect the results. One solution may be to use wavelet decomposition to mitigate the effect of dispersion during data post-processing (see Mirshekari et al. 2018).

- It has been explained in Chapter 2 that judicious placement of accelerometers can improve the quality, and perhaps the accuracy, of the SRP plots. In Chapter 3, then, an irregular configuration was chosen for the placement of the accelerometers. However, more research is needed to determine the optimum number of accelerometers and their configuration.

- This research focuses on locating a single source of vibration. The SRP method is capable of locating multiple sources of vibration using floor vibration measurements (see Chen et al. 2001; Chen et al. 2002).

- The effect of openings in the concrete slab between the sensors and the vibration source on the performance of the SRP method is unknown. More research is needed on this question. 


\section{APPENDIX}

\section{APPENDIX A}

\section{A.1 Localization of White Noise Excitation (Regarding Section 3.6.1)}

The SRP and localization error plots for the tests (listed in Table 3.25) in which the shaker was placed at L2, L3, and L4 are shown below.

Figure A.1 shows the configuration for the two tests when the shaker was placed at L2. Figure A.2 shows the corresponding SRP plots for these tests. Based on visual inspection of the SRP plots with respect to different decaying powers, $b=0.3$ was chosen for tests OHR2nd-L2-1 and OHR2nd-L2-2. It was found that a decaying power of more than 0.3 almost results in the same localization error. In such cases, the A5 accelerometer is surrounded by red. On the other hand, a decaying power of less than 0.3 results in an ambiguous plot. Figure A.3 shows the corresponding localization errors for different WPS. 


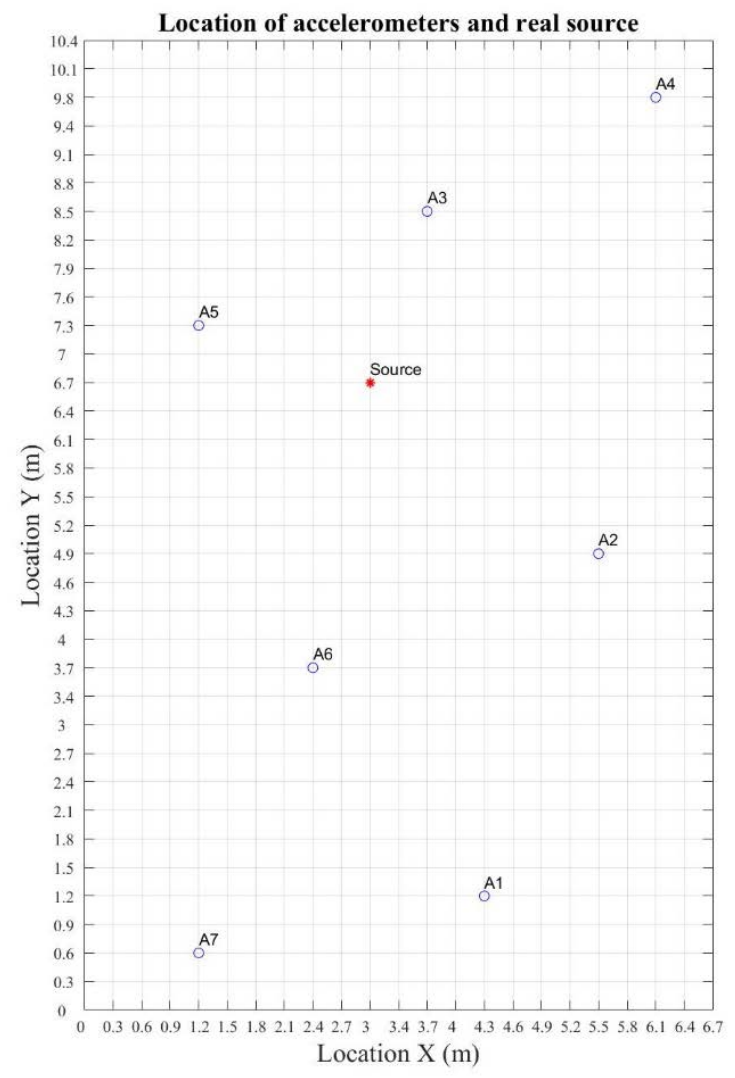

Figure A.1: Test Setup for Tests When the Shaker was Placed at L2

OHR2nd_L2_1.mat

Speed- $X=75 \mathrm{~m} / \mathrm{s} \&$ Speed- $Y=108 \mathrm{~m} / \mathrm{s}$

Error $=1.6 \mathrm{~m}$

Estimated $X=1.5 \mathrm{~m}$ and $Y=7.3 \mathrm{~m} \quad$ Real $X=3 \mathrm{~m}$ and $Y=6.7 \mathrm{~m}$

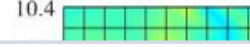

$1^{\text {st }}$ Highest Peak $(E=1.6 \mathrm{~m})$

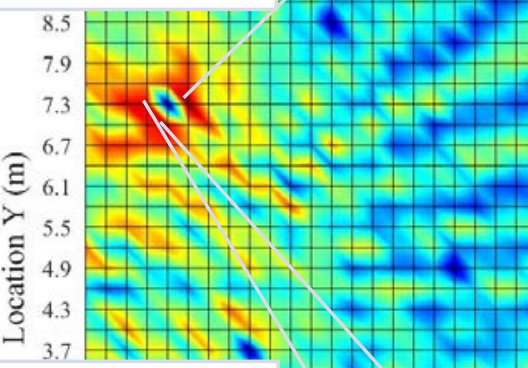

$2^{\text {nd }}$ Highest Peak $(E=2.2 \mathrm{~m})$

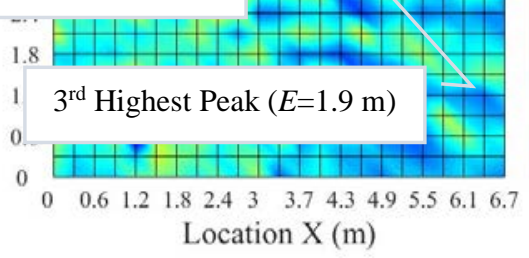

(a) OHR2nd-L2-1

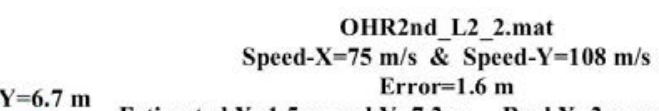

$75 \mathrm{~m} / \mathrm{s}$ \& Speed-Y $=108 \mathrm{~m} / \mathrm{s}$

Estimated $X=1.5 \mathrm{~m}$ and $Y=7.3 \mathrm{~m} \quad$ Real $X=3 \mathrm{~m}$ and $Y=6.7 \mathrm{~m}$

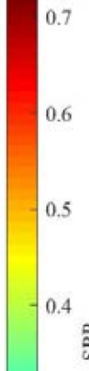

$-0.3$

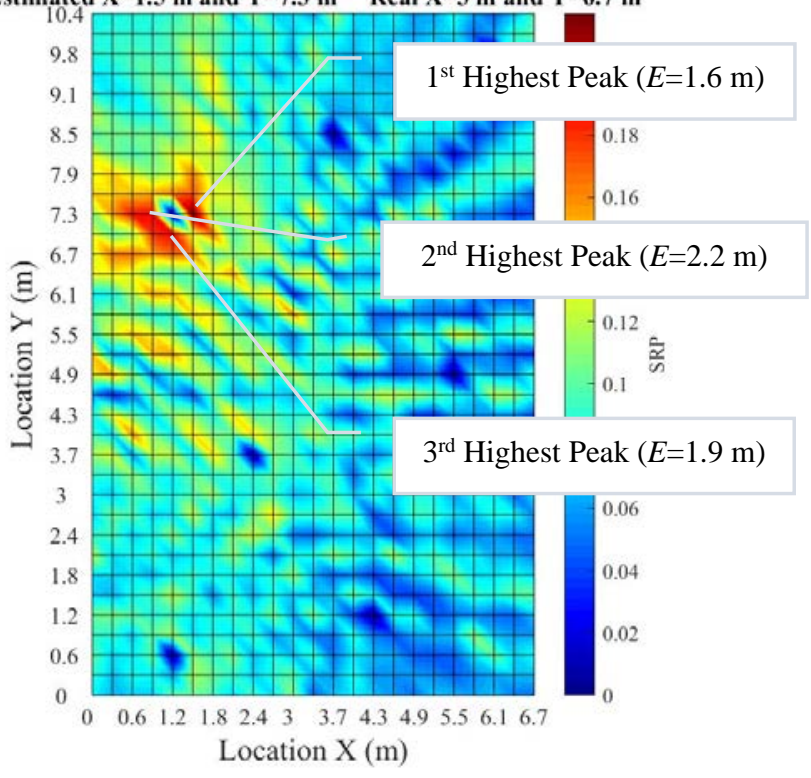

(b) OHR2nd-L2-2 
Figure A.2: SRP Plots When Considering a Two-Second Waveform from 15-17 sec and Filtering in a Frequency Range of $5-200 \mathrm{~Hz}(b=0.3)$

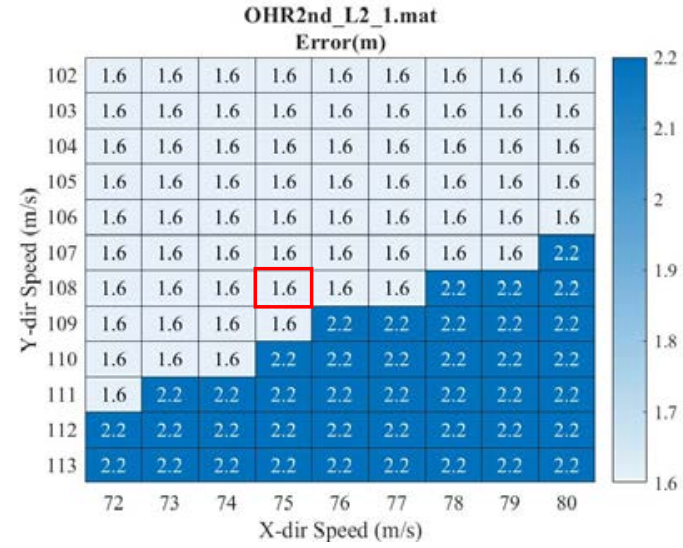

(a) Test OHR2nd-L2-1

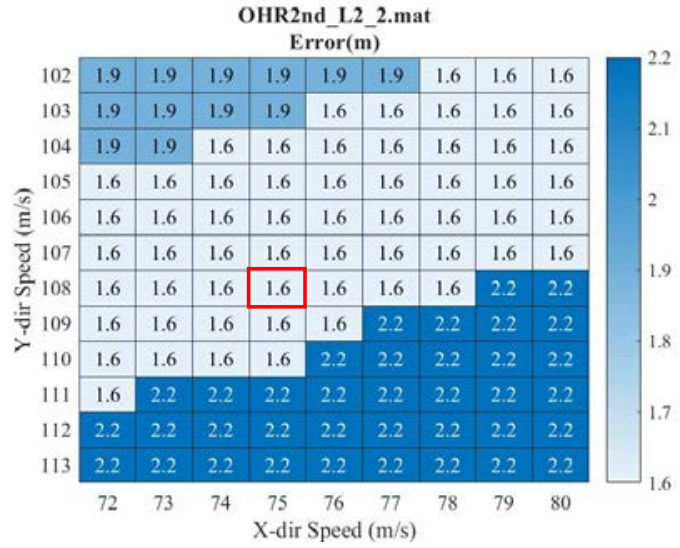

(b) Test OHR2nd-L2-2

Figure A.3: Localization Error Plots with Respect to WPS in X- and Y-Directions When Considering a Two-Second Waveform from 15-17 sec and Filtering in a Frequency Range of 5-200 Hz ( $b=0.3)$

Similarly, Figure A.4 shows the configuration for the tests when the shaker was placed at L3. Figure A.5 shows the SRP plots, and Figure A.6 shows the corresponding localization error plots. 


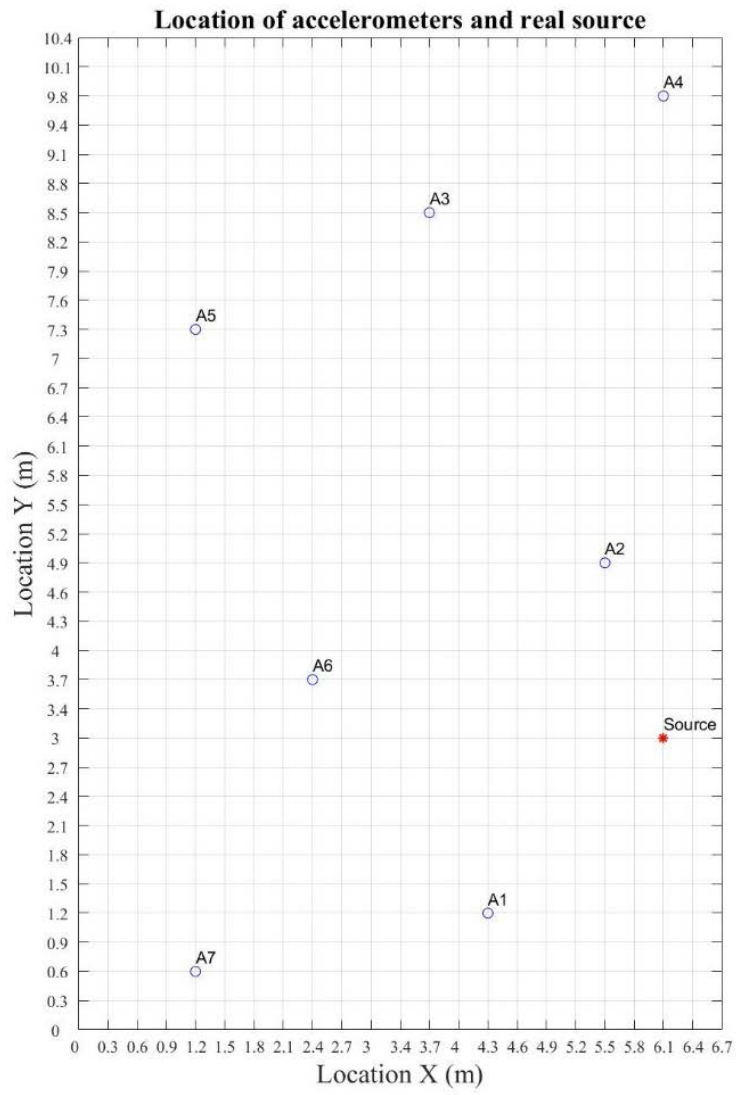

Figure A.4: Test Setup for Tests When the Shaker was Placed at L3 


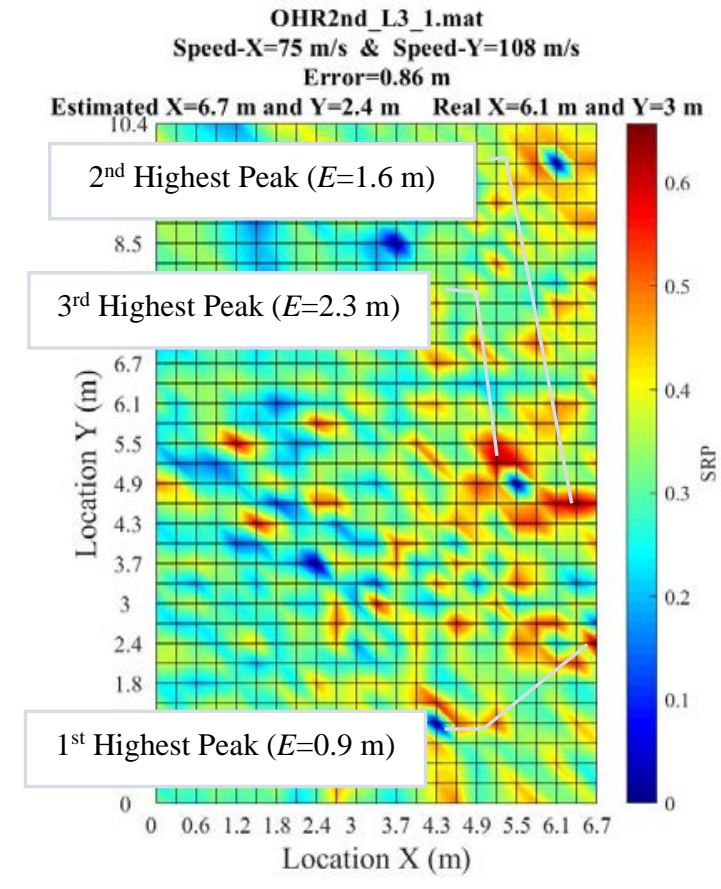

(a) OHR2nd-L3-1

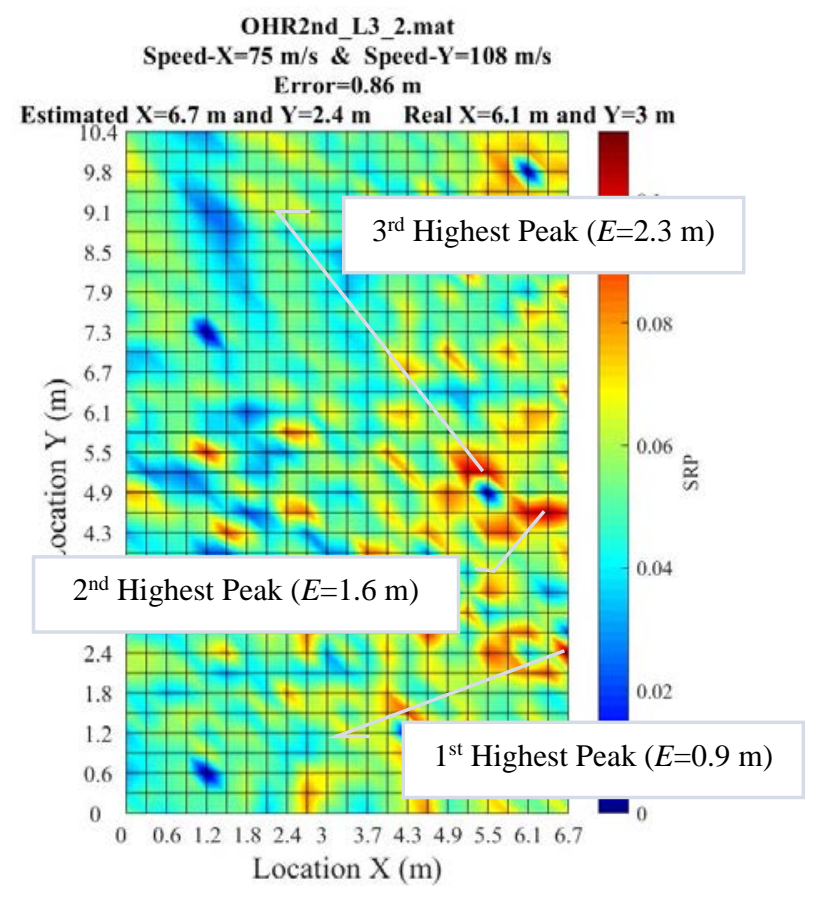

(b) OHR2nd-L3-2

Figure A.5: SRP Plots When Considering a Two-Second Waveform from 15-17 sec and Filtering in a Frequency Range of $5-200 \mathrm{~Hz}(b=0.3)$

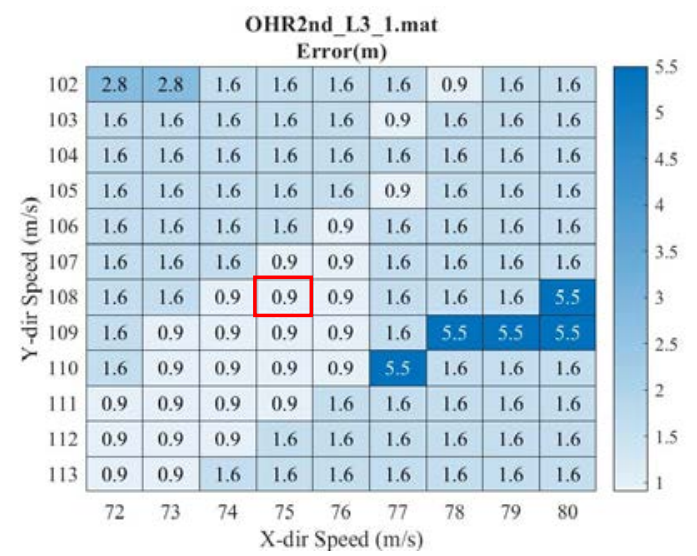

(a) Test OHR2nd-L3-1

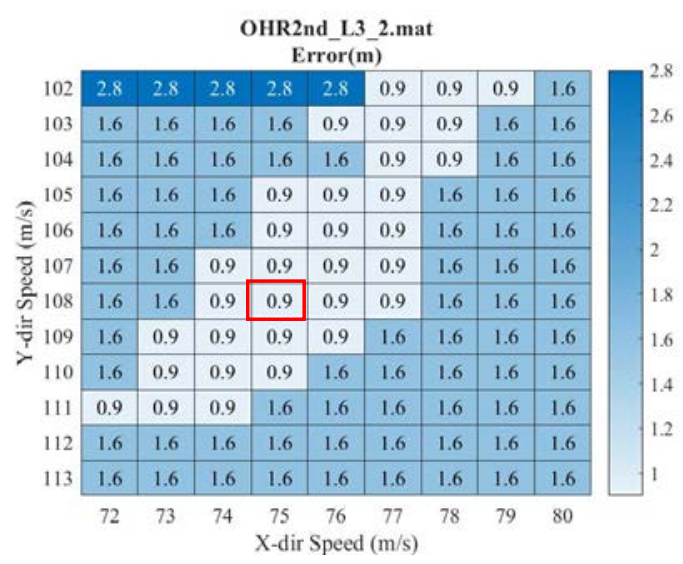

(b) Test OHR2nd-L3-2

Figure A.6: Localization Error Plots with Respect to WPS in X- and Y-Directions When Considering a Two-Second Waveform from 15-20 sec and Filtering in a Frequency Range of $5-200 \mathrm{~Hz}(b=0.3)$ 
Similarly, Figure A.7 shows the configuration for the tests when the shaker was placed at L4; Figure A.8 shows the corresponding SRP plots, and Figure A.9 shows the localization error plots.

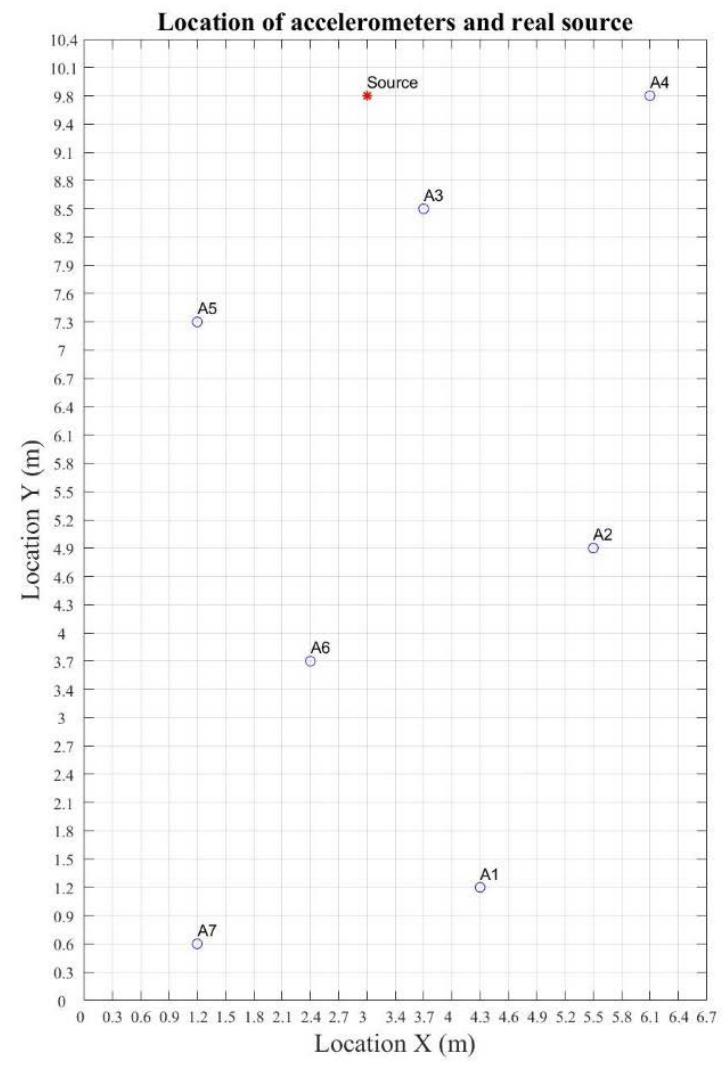

Figure A.7: Test Setup for Tests When the Shaker was Placed at L4 


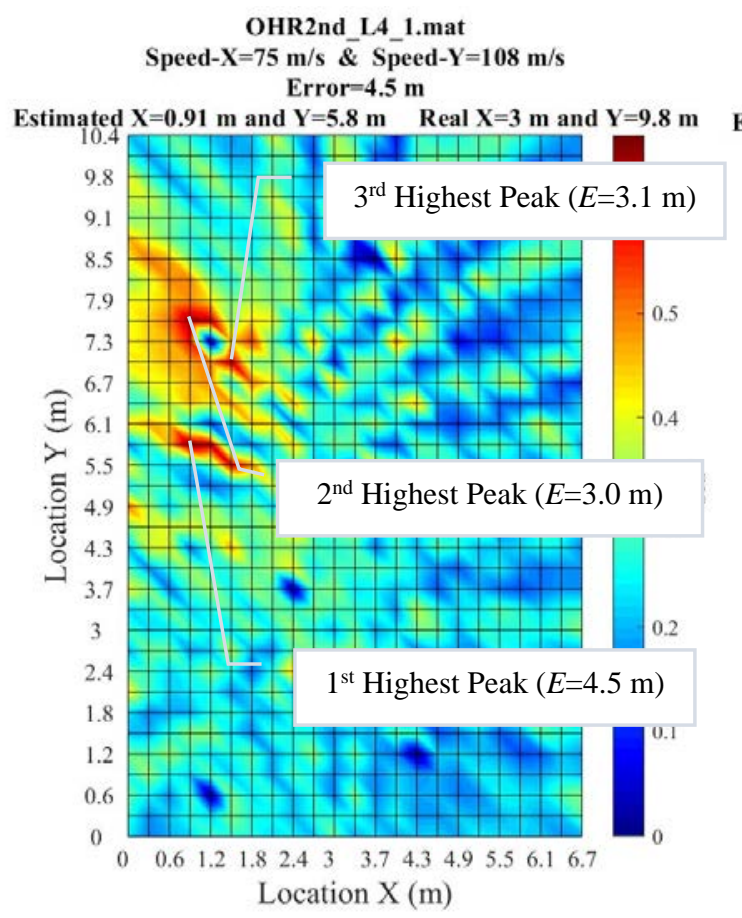

(a) OHR2nd-L4-1

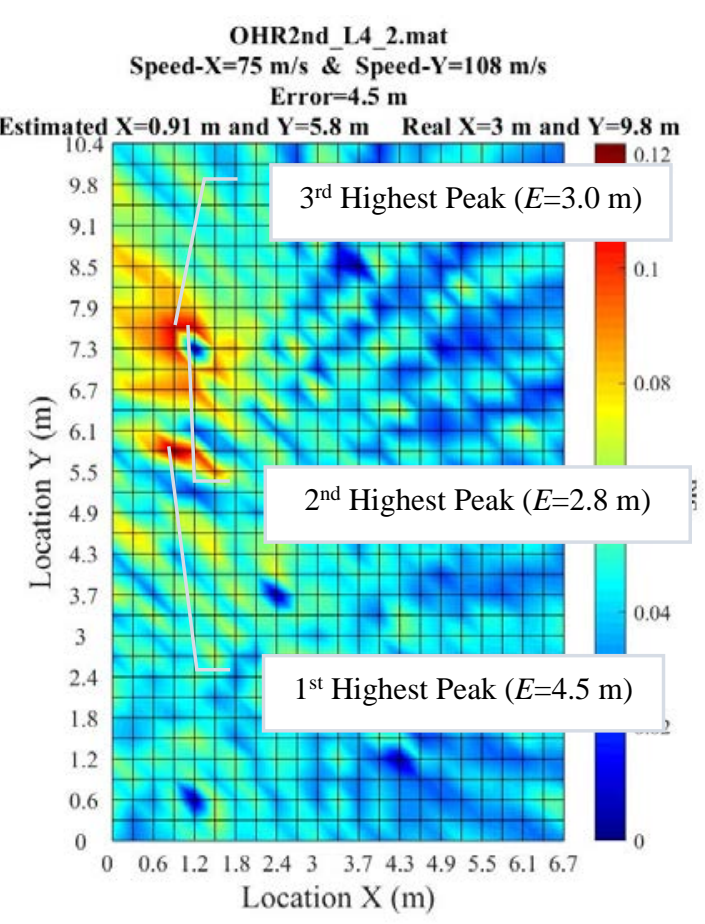

(b) OHR2nd-L4-2

Figure A.8: SRP Plots When Considering a Two-Second Waveform from 15-17 sec and Filtering in a Frequency Range of $5-200 \mathrm{~Hz}(b=0.3)$

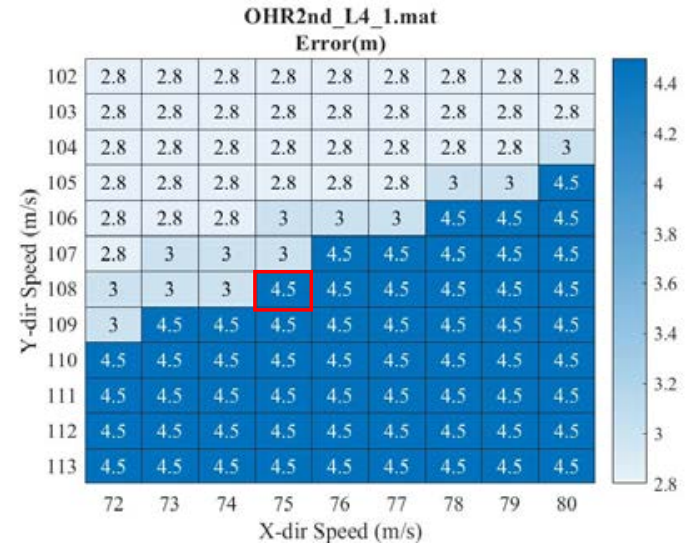

(a) Test OHR2nd-L4-1

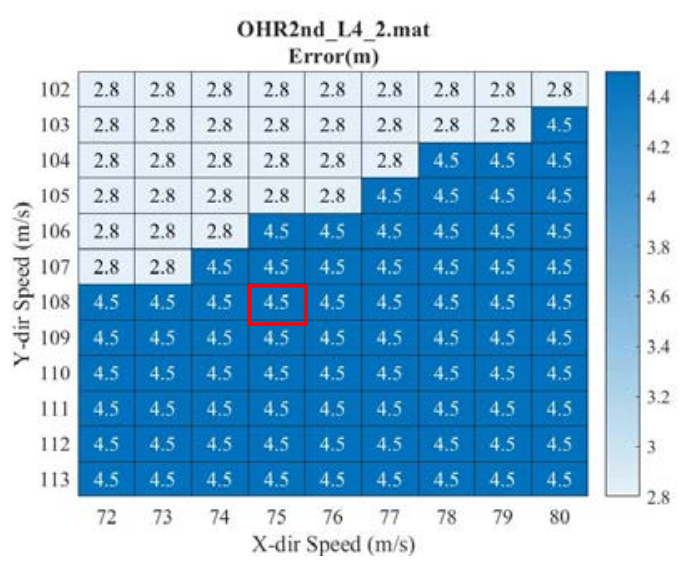

(b) Test OHR2nd-L4-2

Figure A.9: Localization Error Plots with Respect to WPS in X- and Y-Directions When Considering a Two-Second Waveform from 15-20 sec and Filtering in a Frequency Range of $5-200 \mathrm{~Hz}(b=0.3)$ 


\section{A.2 Localization of $70 \mathrm{~Hz}$ Sinusoid Excitation (Regarding Section 3.6.2.1)}

The SRP plots and corresponding localization errors for the tests (listed in Table 3.29) when the shaker was placed at L2, L3, and L4 are shown below.

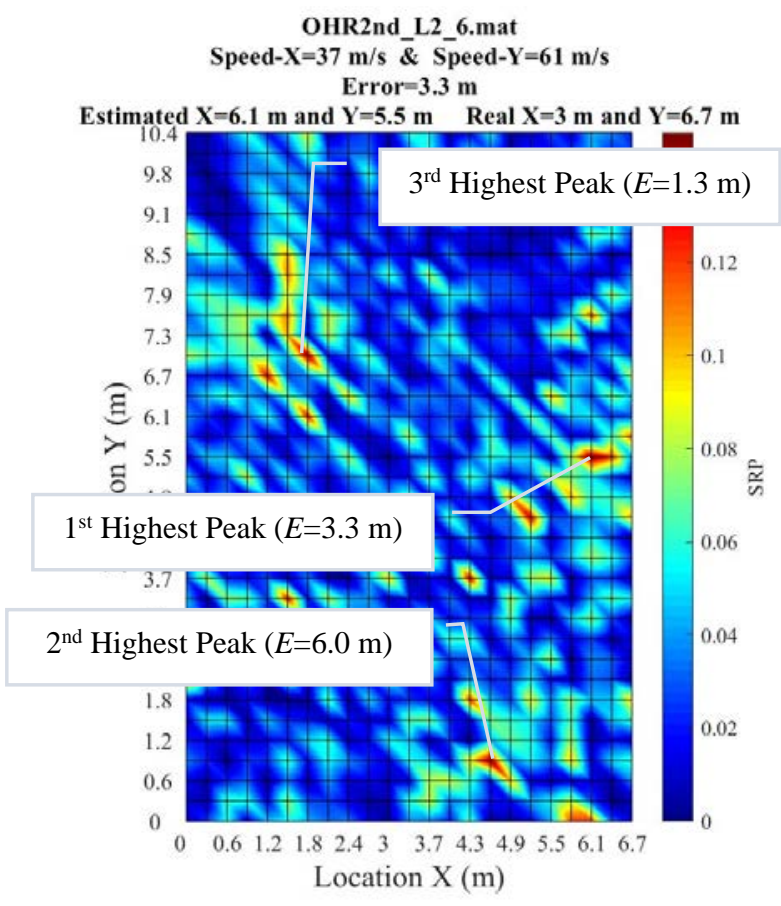

(a) Test OHR2nd-L2-6

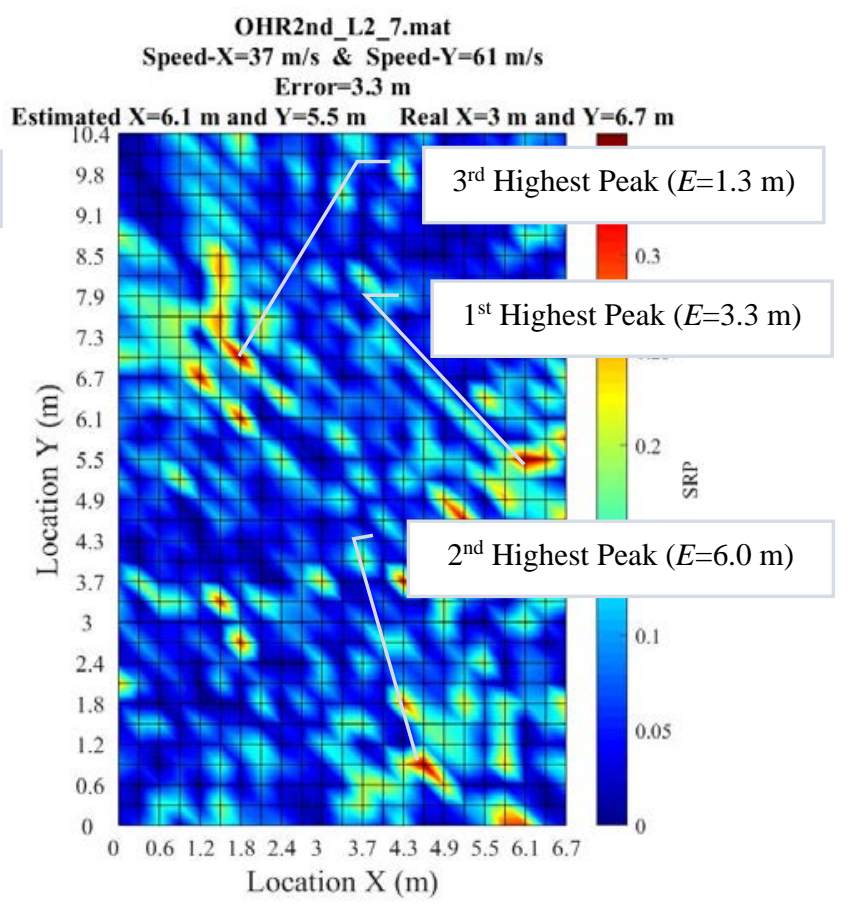

(b) Test OHR2nd-L2-7

Figure A.10: SRP Plots When Considering a Two-Second Waveform from 15-17 sec and Filtering in a Frequency Range of $60-80 \mathrm{~Hz}(b=0.5)$

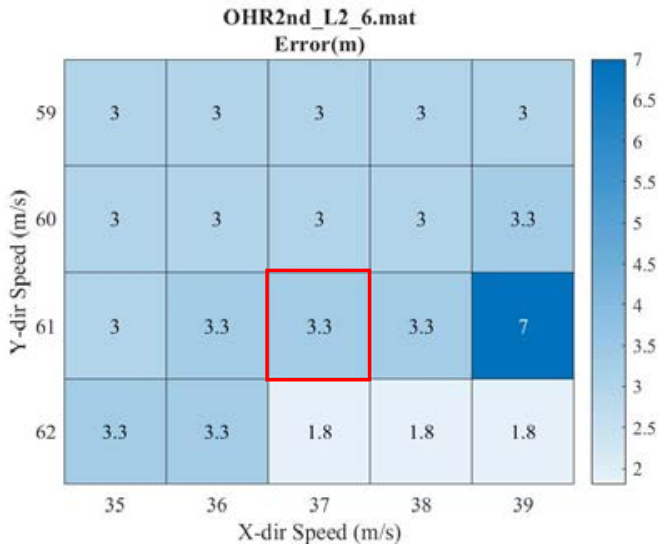

(a) Test OHR2nd-L2-6

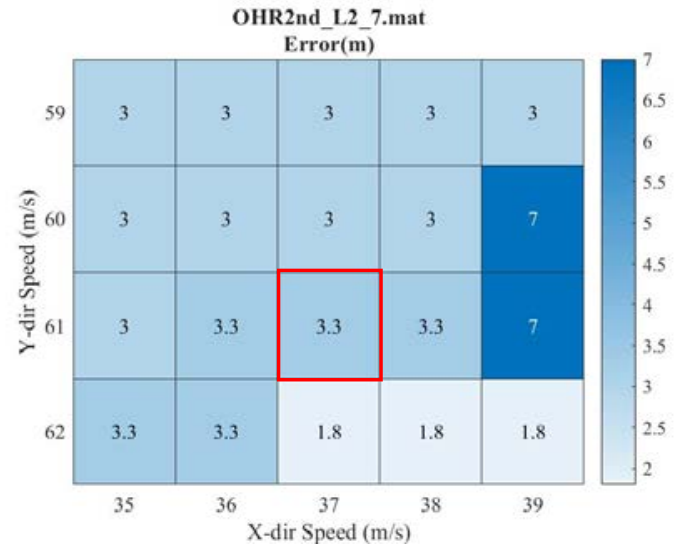

(b) Test OHR2nd-L2-7 
Figure A.11: Localization Error Plots When Considering a Two-Second Waveform from $15-17$ sec and Filtering in a Frequency Range of $60-80 \mathrm{~Hz}(b=0.5)$

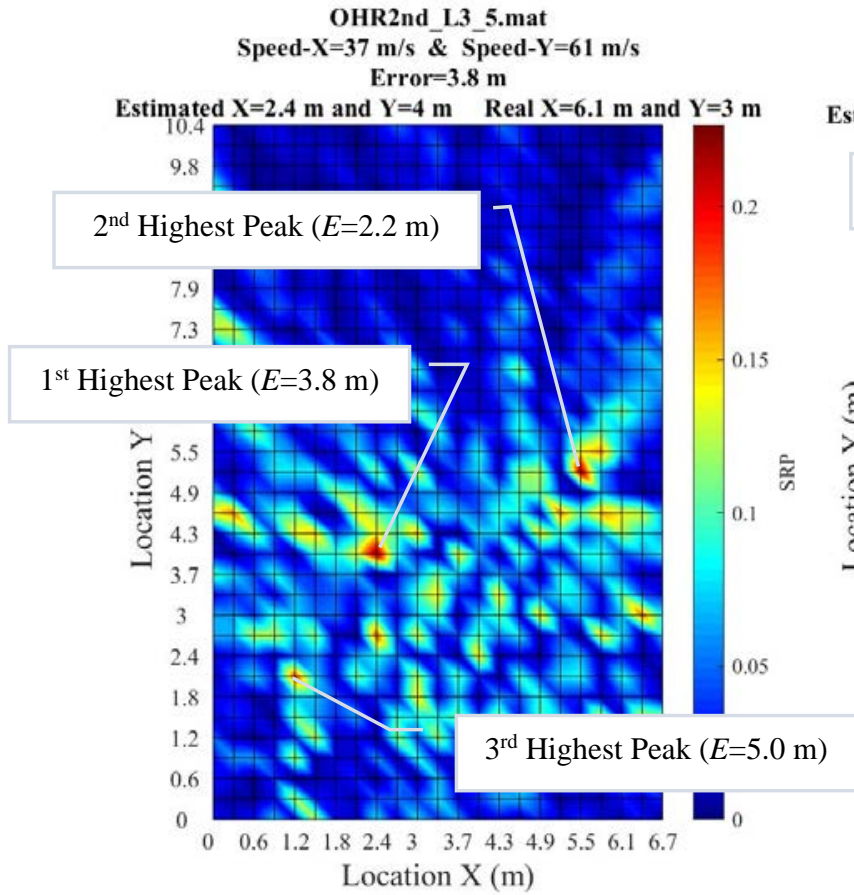

(a) Test OHR2nd-L3-5

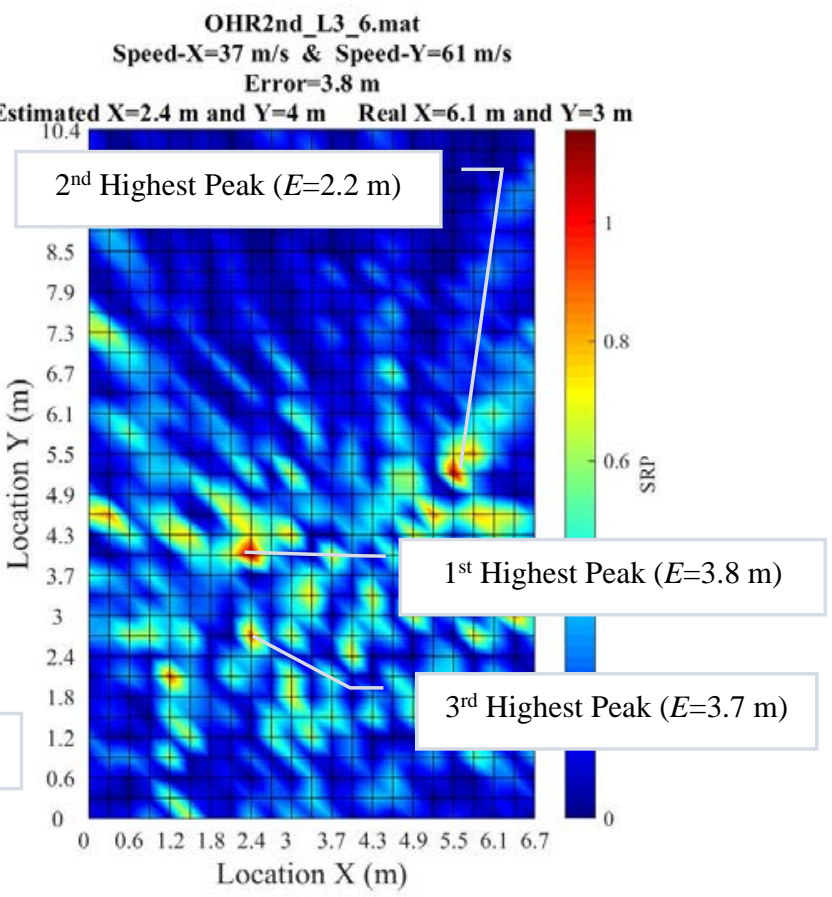

(b) Test OHR2nd-L3-6

Figure A.12: SRP Plots When Considering a Two-Second Waveform from 15-17 sec and Filtering in a Frequency Range of $60-80 \mathrm{~Hz}(b=0.5)$

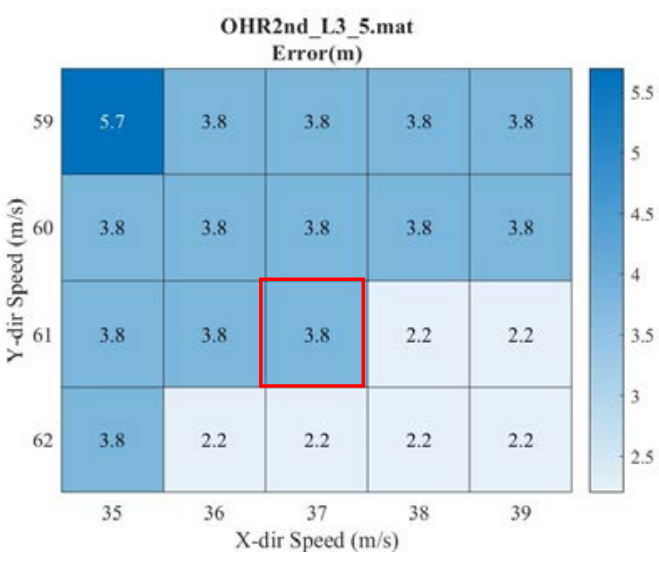

(a) Test OHR2nd-L3-5

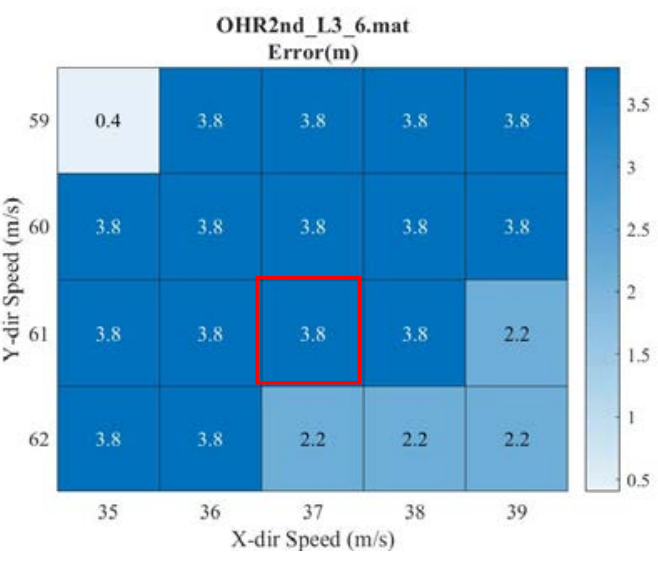

(b) Test OHR2nd-L3-6

Figure A.13: Localization Error Plots When Considering a Two-Second Waveform from $15-17 \mathrm{sec}$ and Filtering in a Frequency Range of 60-80 Hz $(b=0.5)$ 


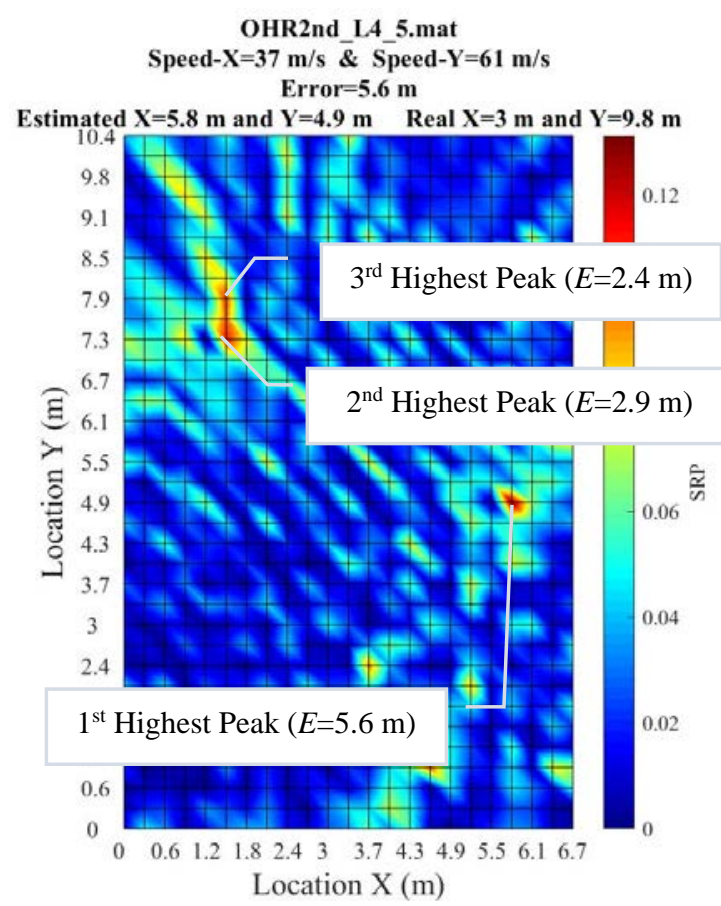

(a) Test OHR2nd-L4-5

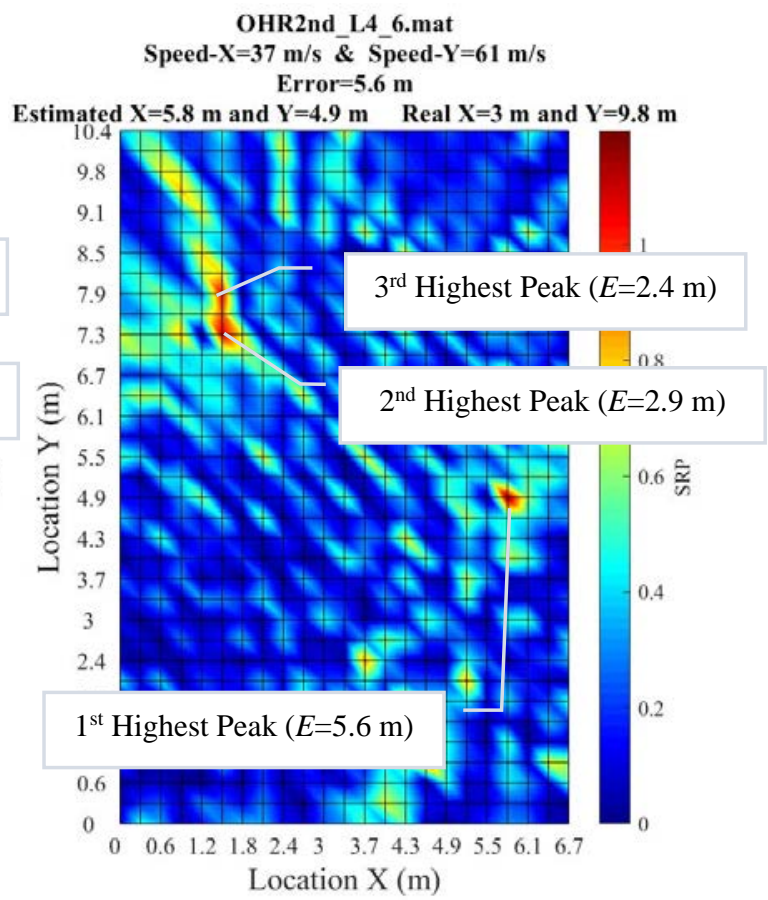

(b) Test OHR2nd-L4-6

Figure A.14: SRP Plots When Considering a Two-Second Waveform from 15-17 sec and Filtering in a Frequency Range of $60-80 \mathrm{~Hz}(b=0.5)$

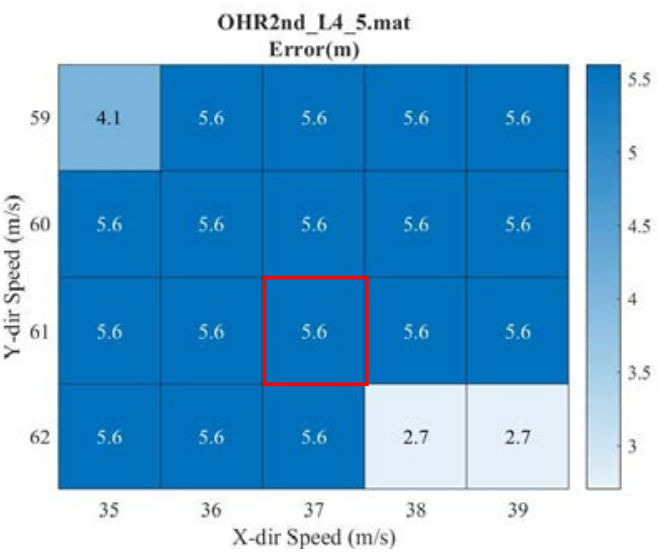

(a) Test OHR2nd-L4-5

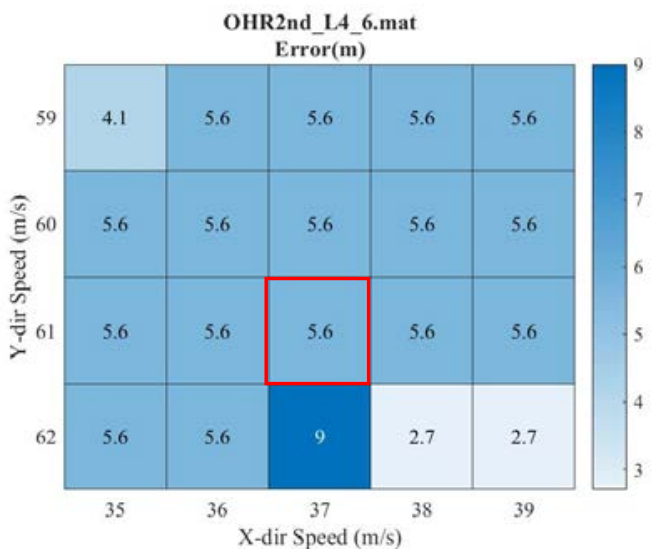

(b) Test OHR2nd-L4-6

Figure A.15: Localization Error Plots When Considering a Two-Second Waveform from $15-17 \mathrm{sec}$ and Filtering in a Frequency Range of $60-80 \mathrm{~Hz}(b=0.5)$ 


\section{A.3 Localization of $90 \mathrm{~Hz}$ Sinusoid Excitation (Regarding Section 3.6.2.2)}

The SRP plots and corresponding localization errors for the tests (listed in Table 3.32) when the shaker was placed at L2, L3, and L4 are shown in the figures below.

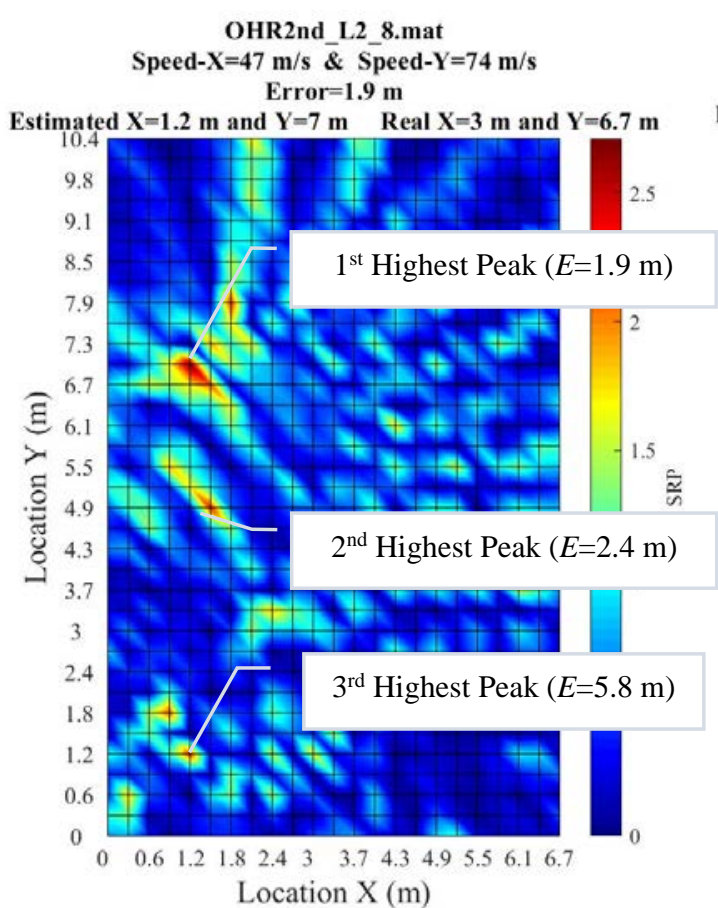

(a) Test OHR2nd-L2-8

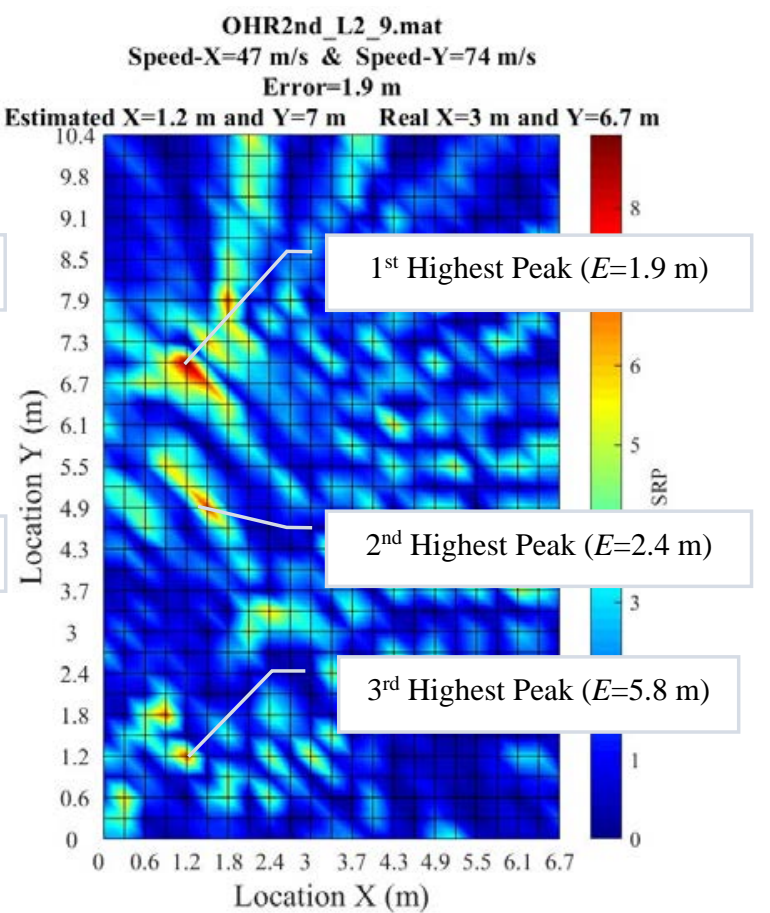

(b) Test OHR2nd-L2-9

Figure A.16: SRP Plots When Considering a Two-Second Waveform from 15-17 sec and Filtering in a Frequency Range of $80-100 \mathrm{~Hz}(b=0.4)$

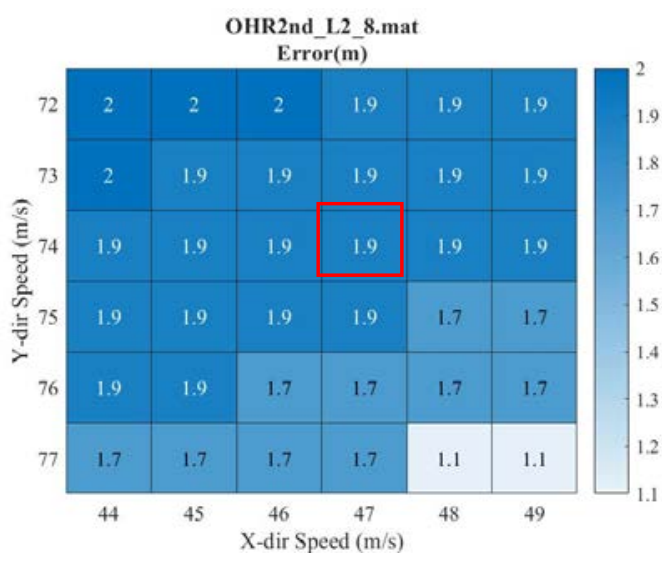

(a) Test OHR2nd-L2-8

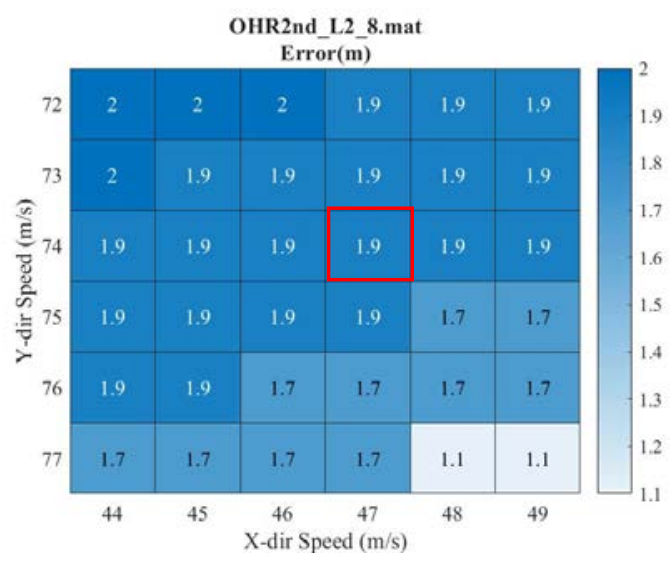

(b) Test OHR2nd-L2-9 
Figure A.17: Localization Error Plots When Considering a Two-Second Waveform from 15-17 sec and Filtering in a Frequency Range of 80-100 Hz $(b=0.4)$

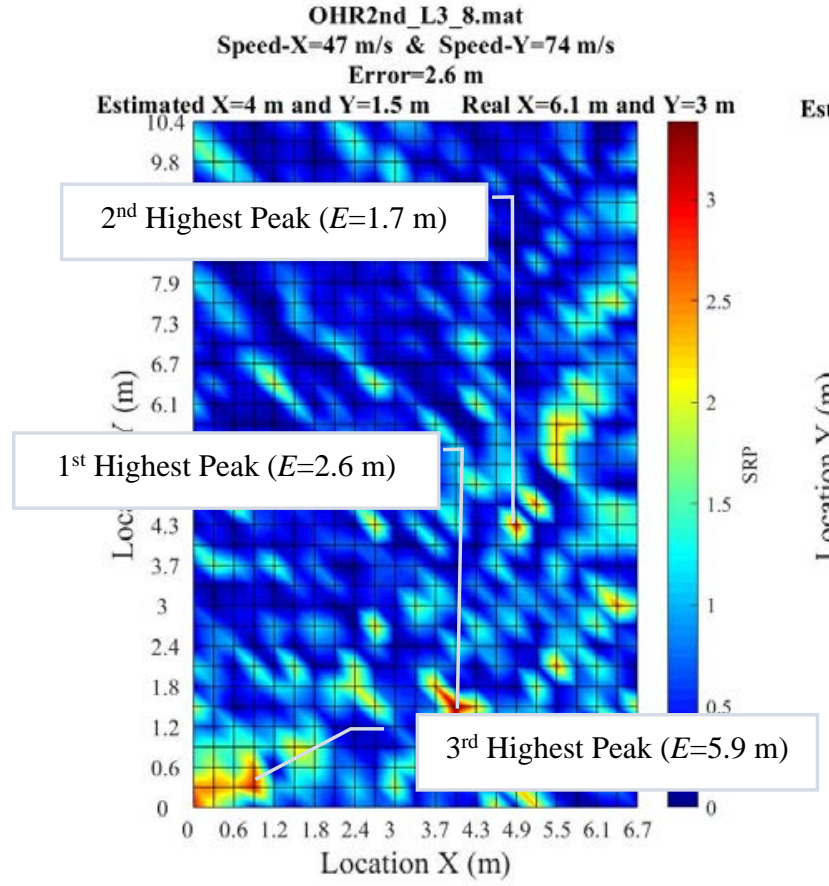

(a) Test OHR2nd-L3-8

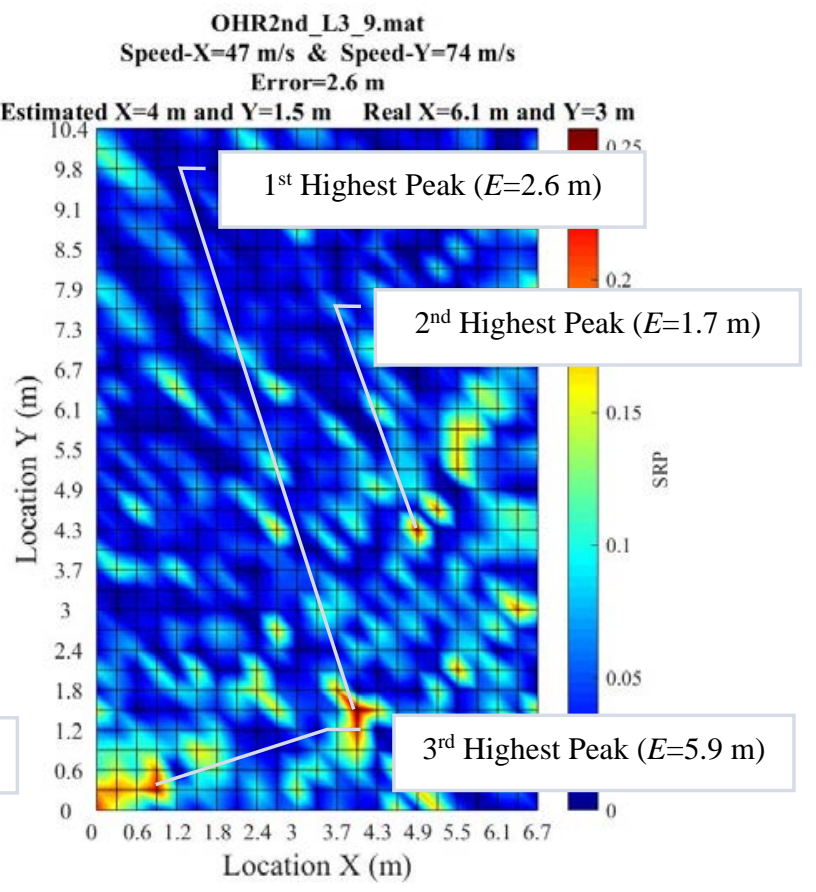

(b) Test OHR2nd-L3-9

Figure A.18: SRP Plots When Considering a Two-Second Waveform from 15-17 sec and Filtering in a Frequency Range of $80-100 \mathrm{~Hz}(b=0.4)$

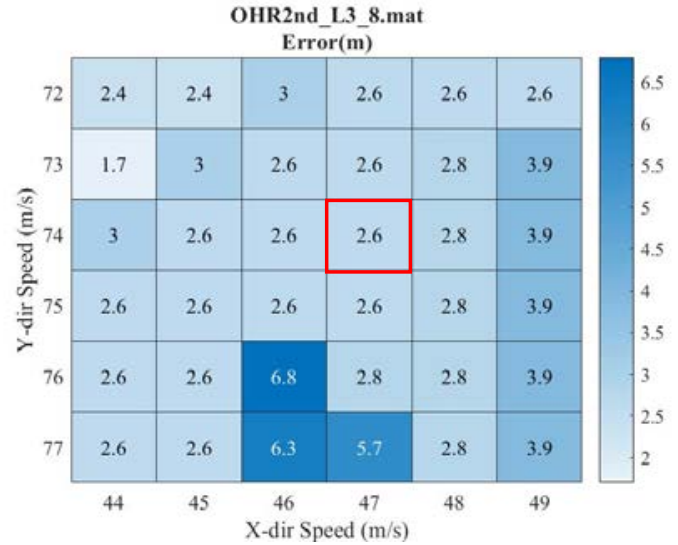

(a) Test OHR2nd-L3-8

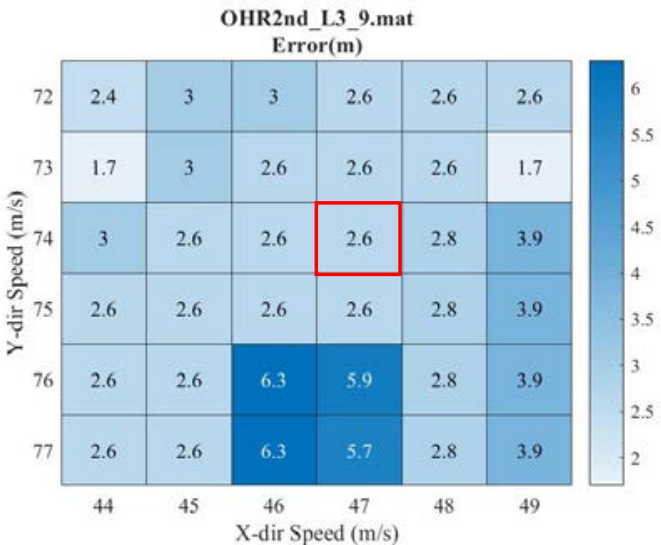

(b) Test OHR2nd-L3-9

Figure A.19: Localization Errors When Considering a Two-Second Waveform from 15-17 sec and Filtering in a Frequency Range of 80-100 Hz ( $b=0.4)$ 


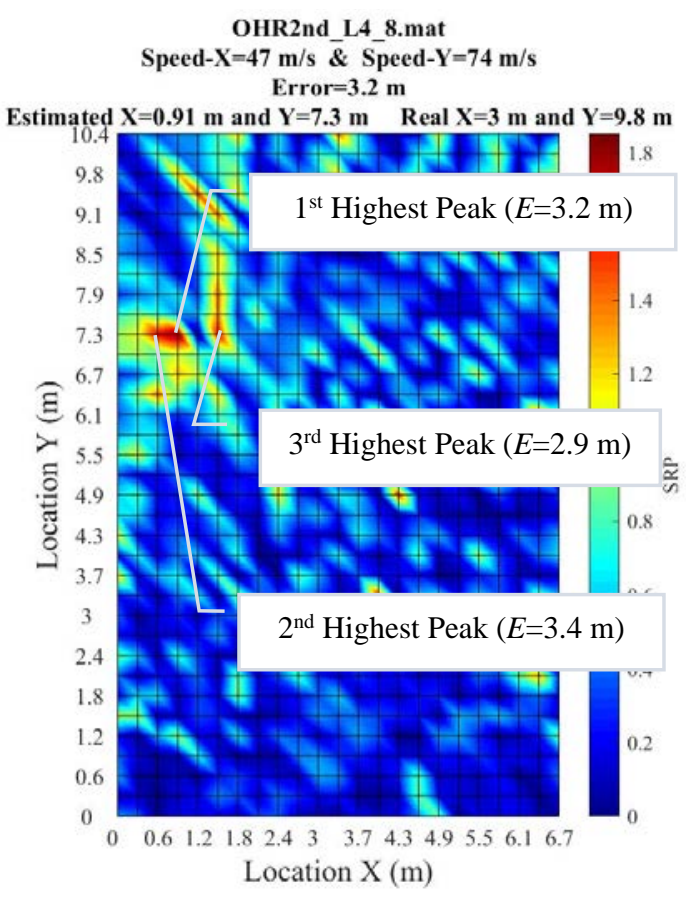

(a) Test OHR2nd-L4-8

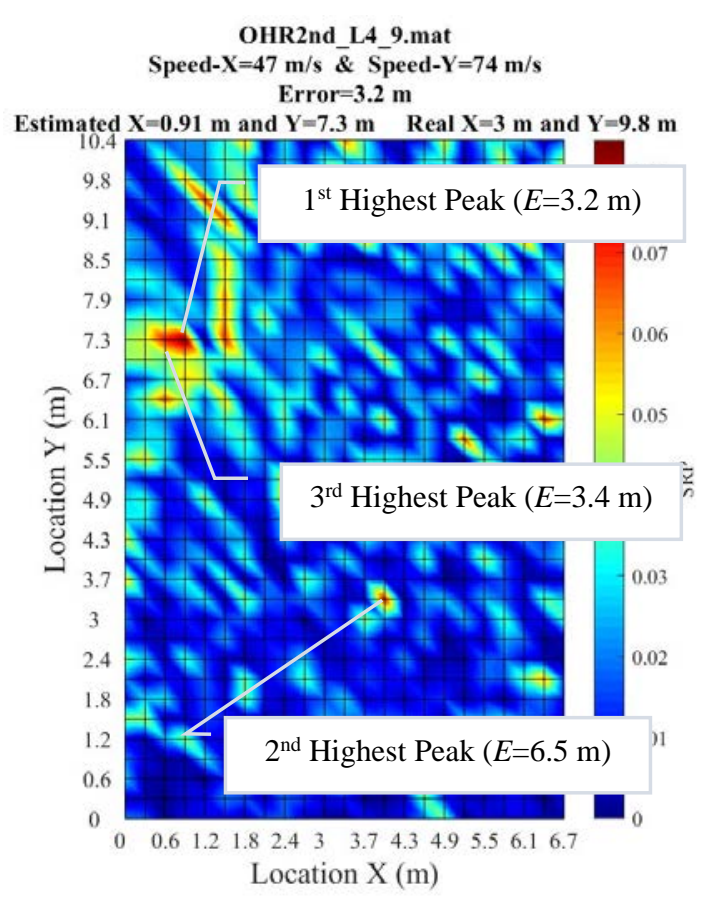

(b) Test OHR2nd-L4-9

Figure A.20: SRP Plots When Considering a Two-Second Waveform from 15-17 sec and Filtering in a Frequency Range of $80-100 \mathrm{~Hz}(b=0.4)$

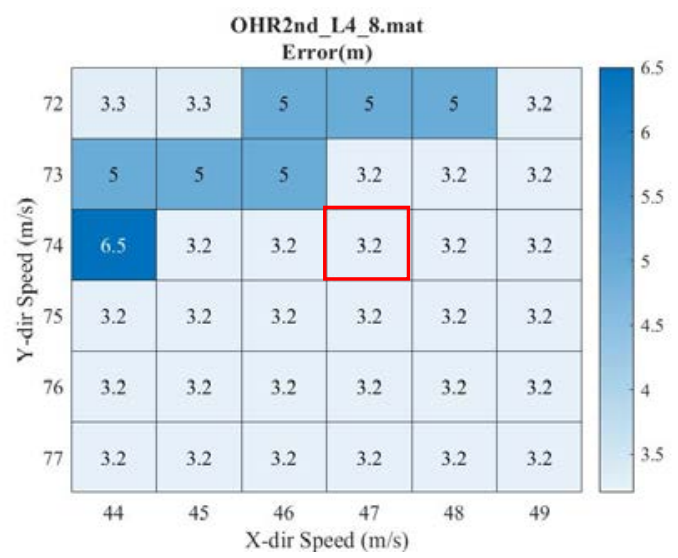

(a) Test OHR2nd-L4-8

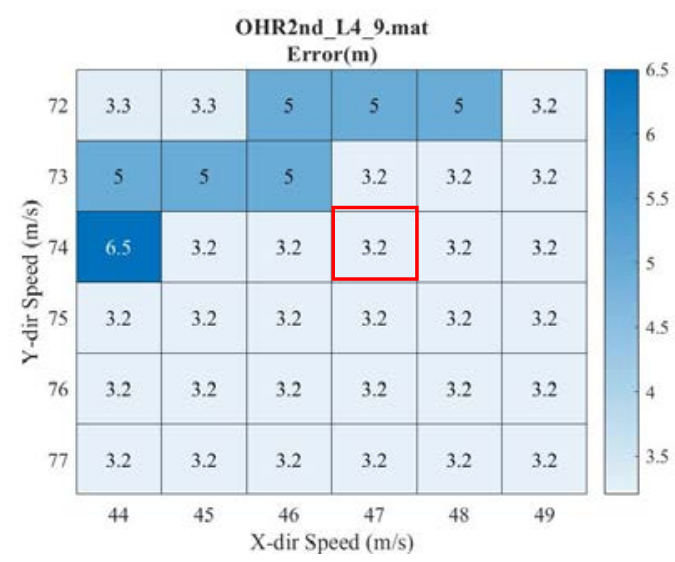

(b) Test OHR2nd-L4-9

Figure A.21: Localization Error Plots When Considering a Two-Second Waveform from $15-17$ sec and Filtering in a Frequency Range of $80-100 \mathrm{~Hz}(b=0.4)$ 


\section{A.4 Localization of $110 \mathrm{~Hz}$ Sinusoid Excitation (Regarding Section 3.6.2.3)}

The SRP and localization error plots for the tests (listed in Table 3.35) in which the shaker was placed at L2, L3, and L4 are shown below.

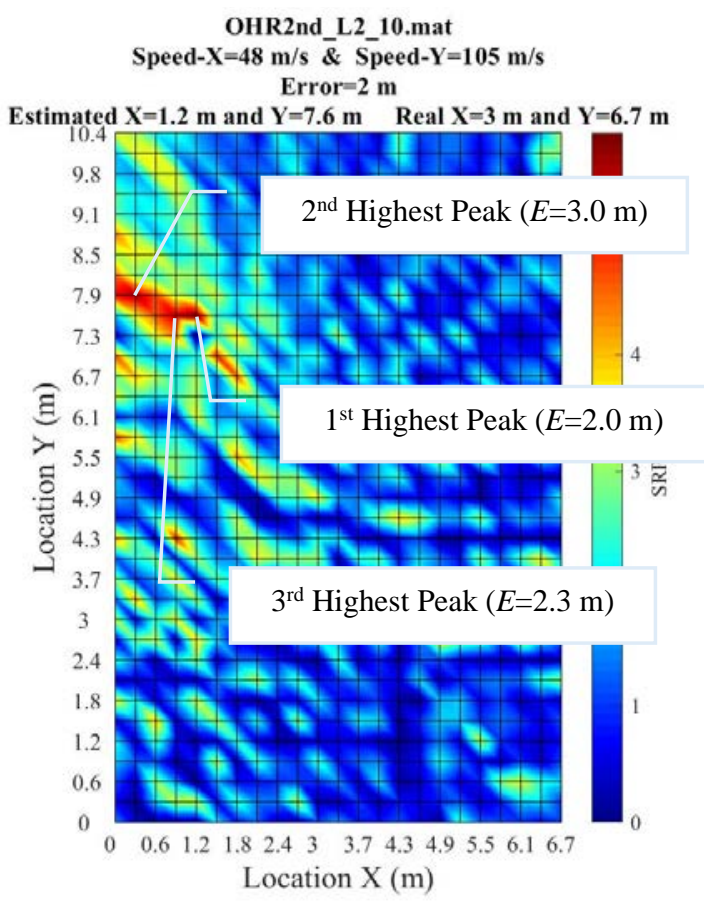

(a) Test OHR2nd_L2_10

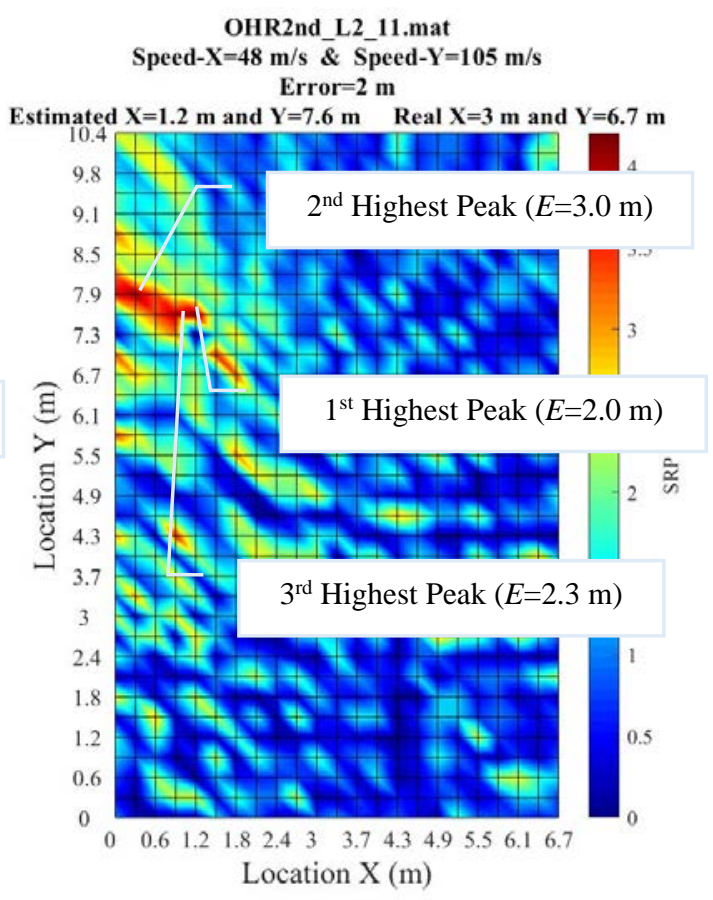

(b) Test OHR2nd_L2_11

Figure A.22: SRP Plots When Considering a Two-Second Waveform from 15-17 sec and Filtering in a Frequency Range of $100-120 \mathrm{~Hz}(b=0.3)$ 


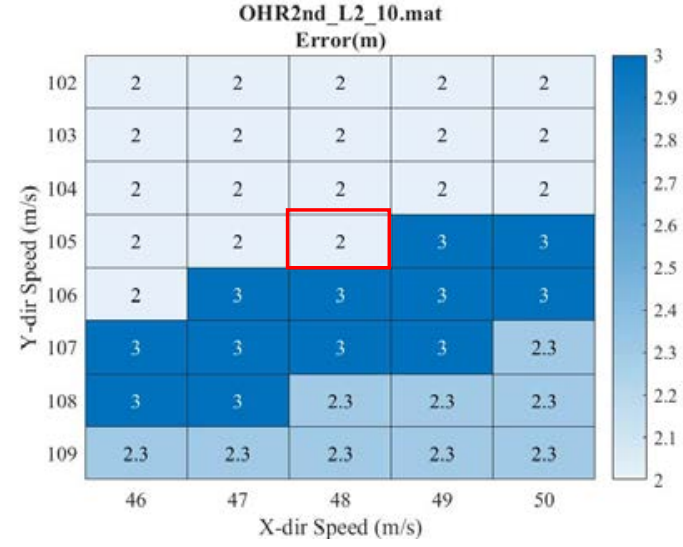

(a) Test OHR2nd_L2_10

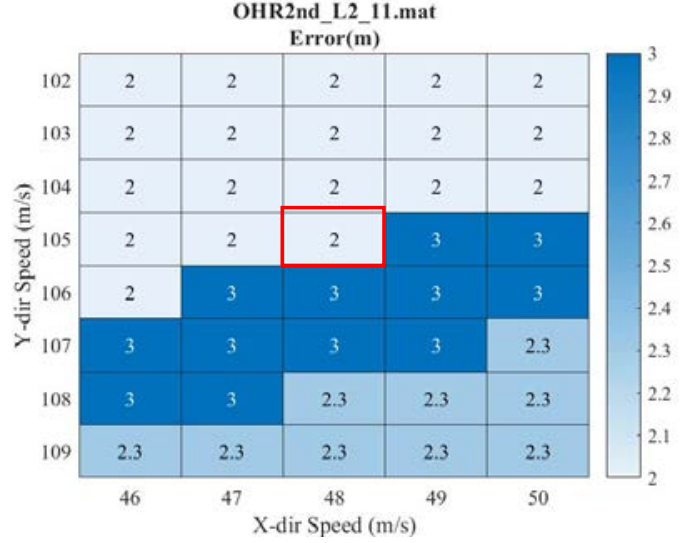

(b) Test OHR2nd_L2_11

Figure A.23: Localization Error Plots When Considering a Two-Second Waveform from $15-17$ sec and Filtering in a Frequency Range of $100-120 \mathrm{~Hz}(b=0.3)$

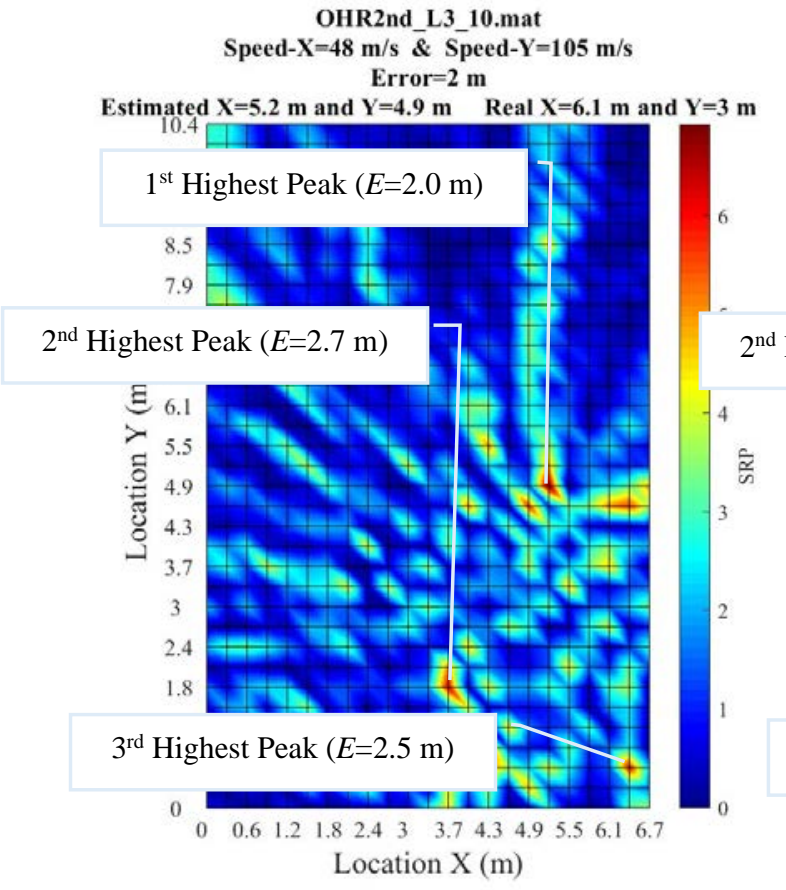

(a) Test OHR2nd_L3_10

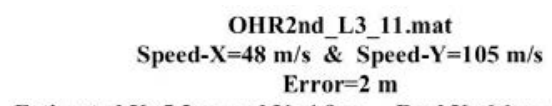

Estimated $X=5.2 \mathrm{~m}$ and $Y=4.9 \mathrm{~m} \quad$ Real $X=6.1 \mathrm{~m}$ and $\mathrm{Y}=3 \mathrm{~m}$ $1^{\text {st }}$ Highest Peak $(E=2.0 \mathrm{~m})$

8.5

7.9

$2^{\text {nd }}$ Highest Peak $(E=2.7 \mathrm{~m})$

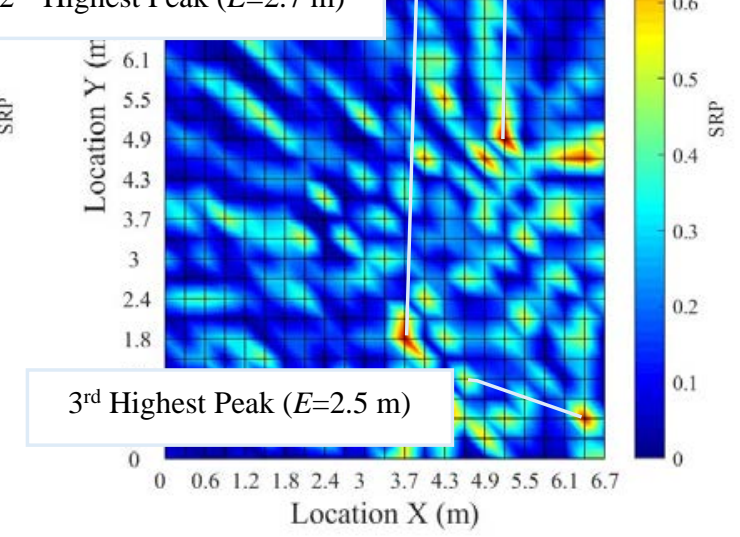

(b) Test OHR2nd_L3_11

Figure A.24: SRP Plots When Considering a Two-Second Waveform from 15-17 sec and Filtering in a Frequency Range of $100-120 \mathrm{~Hz}(b=0.4)$ 


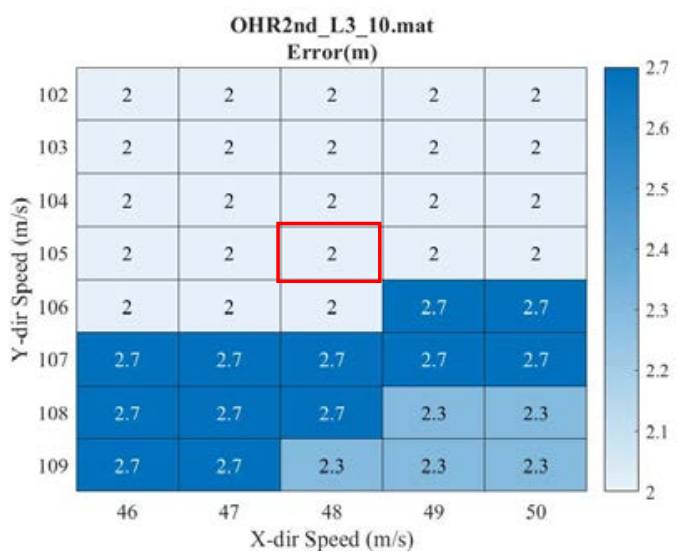

(a) Test OHR2nd_L3_10

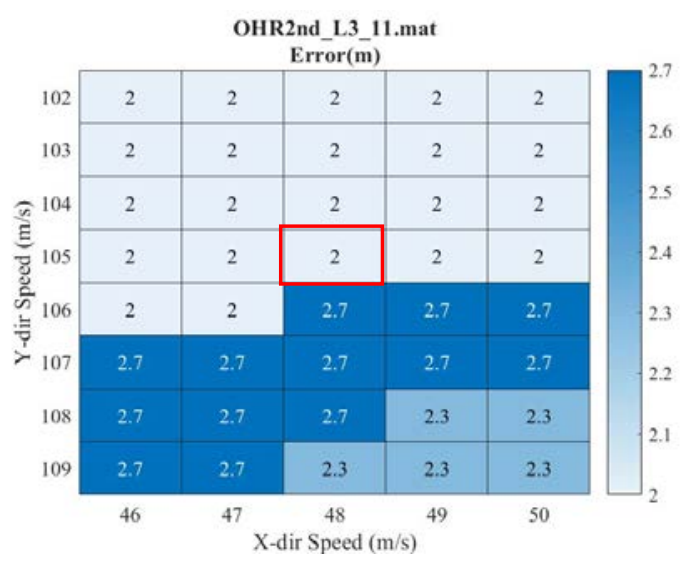

(b) Test OHR2nd_L3_11

Figure A.25: Localization Error Plots When Considering a Two-Second Waveform from $15-17$ sec and Filtering in a Frequency Range of $100-120 \mathrm{~Hz}(b=0.4)$

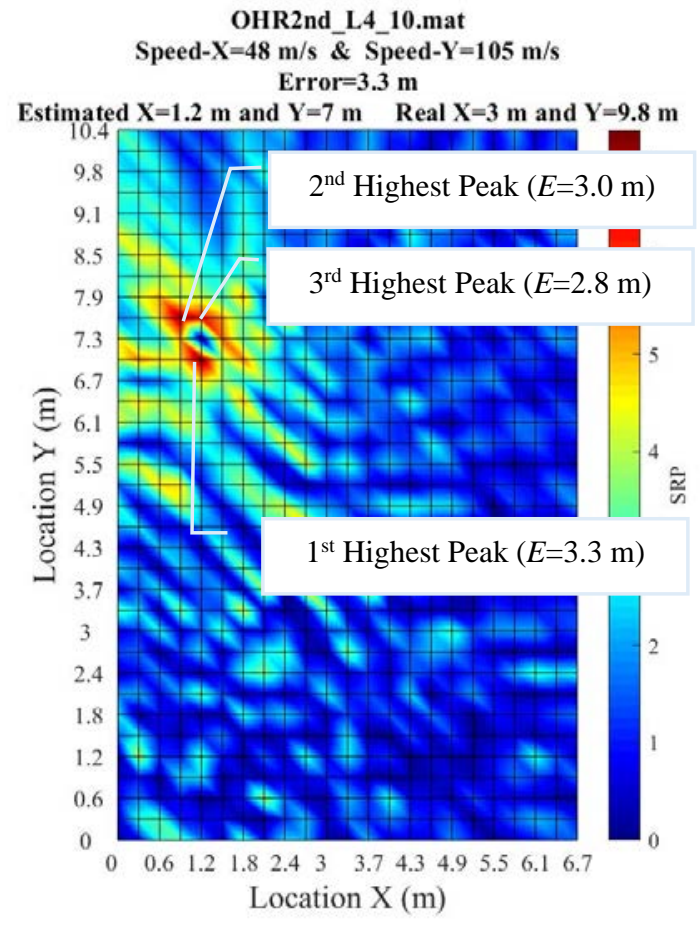

(a) Test OHR2nd_L4_10

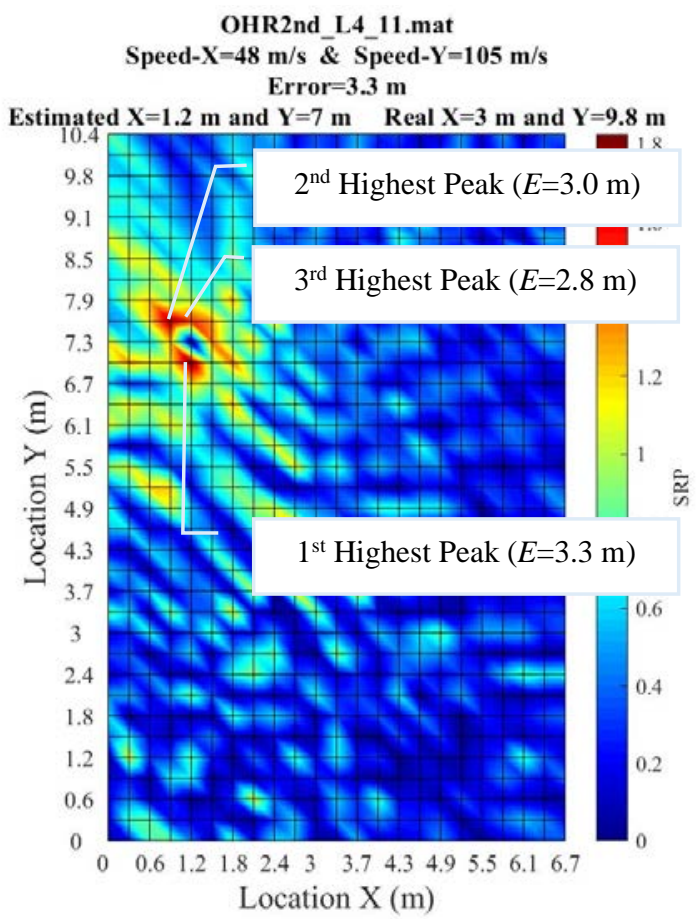

(b) Test OHR2nd_L4_11

Figure A.26: SRP Plots When Considering a Two-Second Waveform from 15-17 sec and Filtering in a Frequency Range of $100-120 \mathrm{~Hz}(b=0.4)$ 


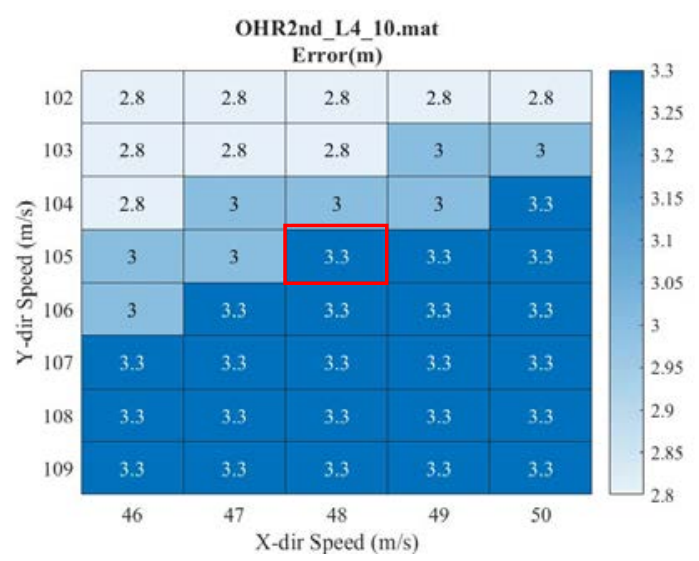

(a) Test OHR2nd_L4_10

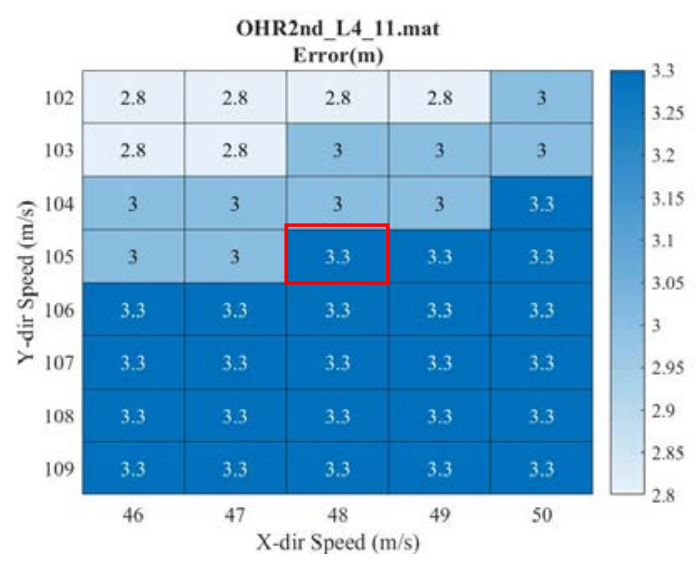

(b) Test OHR2nd_L4_11

Figure A.27: Localization Error Plots When Considering a Two-Second Waveform from $15-17$ sec and Filtering in a Frequency Range of $100-120 \mathrm{~Hz}(b=0.4)$ 


\section{REFERENCES}

Addison, P. S. (2017), The illustrated wavelet transform handbook: introductory theory and applications in science, engineering, medicine and finance. CRC press.

Avitabile, P. (2001), "Experimental modal analysis," Sound and vibration, 35(1), pp. 2031.

Bahroun, R., Michel, O., Frassati, F., Carmona, M. and Lacoume, J.L. (2014), "New algorithm for footstep localization using seismic sensors in an indoor environment," Journal of Sound and Vibration, 333(3), pp.1046-1066.

Barrett, A.R. (2006), "Dynamic testing of in-situ composite floors and evaluation of vibration serviceability using the finite element method,” Virginia Polytechnic Inst and State Univ Blacksburg.

Benesty, J., Chen, J. and Huang, Y. (2004), “Time-delay estimation via linear interpolation and cross correlation," IEEE Transactions on speech and audio processing, 12(5), pp.509519.

Benesty, J., Chen, J. and Huang, Y. (2008), Microphone array signal processing (Vol. 1). Springer Science \& Business Media.

Brownjohn, J.M., Pavic, A. and Omenzetter, P. (2004), “A spectral density approach for modelling continuous vertical forces on pedestrian structures due to walking," Canadian Journal of Civil Engineering, 31(1), pp.65-77.

Budi, S., Hyoungseop, K., Kooi, T.J. and Seiji, I. (2009), "Real time tracking and identification of moving persons by using a camera in outdoor environment."

Chan, Y.T. and Ho, K.C. (1994), "A simple and efficient estimator for hyperbolic location,” IEEE Transactions on signal processing, 42(8), pp.1905-1915.

Chen, J.C., Yao, K. and Hudson, R.E. (2002), "Source localization and beamforming,” IEEE Signal Processing Magazine, 19(2), pp.30-39.

Chen, J.C., Hudson, R.E. and Yao, K. (2001), November. "Joint maximum-likelihood source localization and unknown sensor location estimation for near-field wideband signals," In Advanced Signal Processing Algorithms, Architectures, and Implementations XI (Vol. 4474, pp. 521-532). International Society for Optics and Photonics.

Davis, B.T., Caicedo, J.M. and Hirth, V.A. (2021), "Force Estimation and Event Localization (FEEL) of Impacts Using Structural Vibrations," Journal of Engineering Mechanics, 147(3), p.04020154.

DiBiase, J.H., Silverman, H.F. and Brandstein, M.S. (2001), "Robust localization in reverberant rooms,” In Microphone arrays (pp. 157-180). Springer, Berlin, Heidelberg.

Drira, S., Reuland, Y., Pai, S.G., Noh, H.Y. and Smith, I.F. (2019), "Model-based occupant tracking using slab-vibration measurements,” Frontiers in Built Environment, 5, p.63. 
Drira, S., Reuland, Y., Olsen, N.F., Pai, S.G. and Smith, I.F. (2019), November. "Occupant-detection strategy using footstep-induced floor vibrations," In Proceedings of the 1st ACM International Workshop on Device-Free Human Sensing (pp. 31-34).

Dmochowski, J.P., Benesty, J. and Affes, S. (2007), “A generalized steered response power method for computationally viable source localization," IEEE Transactions on Audio, Speech, and Language Processing, 15(8), pp.2510-2526.

Dondurur, D., 2018. “Acquisition and processing of marine seismic data,” Elsevier.

Donohue, K.D., Agrinsoni, A. and Hannemann, J. (2007), “Audio signal delay estimation using partial whitening,” In Proceedings 2007 IEEE SoutheastCon, pp. 466-471

Ebrahimpour, A. and Sack, R.L. (2005), "A review of vibration serviceability criteria for floor structures,” Computers \& Structures, 83(28-30), pp.2488-2494.

Farnell, G.W. (1970), “Properties of elastic surface waves,” Physical acoustics, 6, pp.109166.

Feng, Z.G., Yiu, K.F.C. and Nordholm, S.E. (2011), "Placement design of microphone arrays in near-field broadband beamformers," IEEE transactions on signal processing, 60(3), pp.1195-1204.

Geiger, L. (1912), "Probability method for the determination of earthquake epicenters from the arrival time only,” Bull. St. Louis Univ, 8(1), 56-71.

Gao, G.Y., Song, J. and Yang, J. (2014), “Identifying boundary between near field and far field in ground vibration caused by surface loading," Journal of Central South University, 21(8), pp.3284-3294.

GAO Guang-yun (1998), Theory and application of discontinuous barriers [D]. Hangzhou: Department of Civil Engineering, Zhejiang University.

Harris, F.J. (1978), “On the use of windows for harmonic analysis with the discrete Fourier transform,” Proceedings of the IEEE, 66(1), pp. 51-83.

Haupt, W.A. (1981), “Model tests on screening of surface waves,” In Proceedings of Tenth International Conference on Soil Mechanics and Foundation Engineering, San Francisco, 1981.

Havskov, J., Bormann, P. and Schweitzer, J. (2009), "Earthquake location,” In New Manual of Seismological Observatory Practice (NMSOP) (pp. 1-28). Deutsches GeoForschungsZentrum GFZ.

Hormozabad, S.J. and Soto, M.G. (2021), "Real-time damage identification of discrete structures via neural networks subjected to dynamic loading," In Health Monitoring of Structural and Biological Systems XV (Vol. 11593, p. 115932O). International Society for Optics and Photonics.

Kennett, B.L., Engdahl, E.R. and Buland, R. (1995), “Constraints on seismic velocities in the Earth from traveltimes,” Geophysical Journal International, 122(1), pp.108-124. 
Kim, D.S. and Lee, J.S. (2000), "Propagation and attenuation characteristics of various ground vibrations,” Soil dynamics and Earthquake engineering, 19(2), pp.115-126.

Knapp, C. and Carter, G. (1976), “The generalized correlation method for estimation of time delay," IEEE transactions on acoustics, speech, and signal processing, 24(4), pp. 320-327

Lam, M., Mirshekari, M., Pan, S., Zhang, P. and Noh, H.Y. (2016), "Robust occupant detection through step-induced floor vibration by incorporating structural characteristics," In Dynamics of Coupled Structures, Volume 4 (pp. 357-367). Springer, Cham.

Lamb, H. (1881), On the vibrations of an elastic sphere. Proceedings of the London Mathematical Society, 1(1), pp.189-212.

Lee, H., Park, J.W. and Helal, A.S. (2009), "September. Estimation of indoor physical activity level based on footstep vibration signal measured by mems accelerometer in smart home environments," In International Workshop on Mobile Entity Localization and Tracking in GPS-less Environments (pp. 148-162). Springer, Berlin, Heidelberg.

Lee, Y.H. and Oh, T. (2016), "The measurement of P-, S-, and R-wave velocities to evaluate the condition of reinforced and prestressed concrete slabs," Advances in Materials Science and Engineering, 2016.

Lysmer, J.F.E.R. and Richart Jr, F.E. (1966), "Dynamic response of footings to vertical loading,” Journal of the Soil Mechanics and Foundations Division, 92(1), pp.65-91.

Mirshekari, M., Fagert, J., Pan, S., Zhang, P. and Noh, H.Y. (2021), “Obstruction-invariant occupant localization using footstep-induced structural vibrations," Mechanical Systems and Signal Processing, 153, p.107499.

Mirshekari, M., Pan, S., Fagert, J., Schooler, E.M., Zhang, P. and Noh, H.Y. (2018), "Occupant localization using footstep-induced structural vibration,” Mechanical Systems and Signal Processing, 112, pp.77-97.

Mirshekari, M., Pan, S., Zhang, P. and Noh, H.Y. (2016), April. "Characterizing wave propagation to improve indoor step-level person localization using floor vibration," In Sensors and smart structures technologies for civil, mechanical, and aerospace systems 2016 (Vol. 9803, p. 980305). International Society for Optics and Photonics.

Niu, R., Vempaty, A. and Varshney, P.K. (2018), "Received-signal-strength-based localization in wireless sensor networks,” Proceedings of the IEEE, 106(7), pp.1166-1182.

Murray, T.M., Allen, D.E., Ungar, E.E. and Davis, D.B. (2016), "Vibrations of steelframed structural systems due to human activity," American Institute of Steel Construction.

Ohlsson, S. (1982), “Floor vibration and human discomfort,” PhD Thesis, Division of Steel and Timber Structures. Chalmers University of Technology, Goteberg Sweden.

O'Keefe, B. (2017), "Finding Location with Time of Arrival and Time Difference of Arrival Techniques,” ECE Senior Capstone Project. 
Omologo, M. and Svaizer, P. (1994), "Acoustic event localization using a crosspowerspectrum phase based technique,” In Proceedings of ICASSP'94. IEEE International Conference on Acoustics, Speech and Signal Processing (Vol. 2, pp. II-273). IEEE

Omologo, M. and Svaizer, P. (1997), "Use of the crosspower-spectrum phase in acoustic event location,” IEEE Transactions on Speech and Audio Processing, 5(3), pp. 288-292

Pan, S., Bonde, A., Jing, J., Zhang, L., Zhang, P. and Noh, H.Y. (2014), April. "Boes: building occupancy estimation system using sparse ambient vibration monitoring," In Sensors and smart structures technologies for civil, mechanical, and aerospace systems 2014 (Vol. 9061, p. 90611O). International Society for Optics and Photonics.

Pan, S., Yu, T., Mirshekari, M., Fagert, J., Bonde, A., Mengshoel, O.J., Noh, H.Y. and Zhang, P. (2017), "Footprintid: Indoor pedestrian identification through ambient structural vibration sensing," Proceedings of the ACM on Interactive, Mobile, Wearable and Ubiquitous Technologies, 1(3), pp.1-31.

Pavic, A. and Zivanovic, S. (2007), September. "Key elements for probabilistic framework for estimation of structural vibration due to human-structure dynamic interaction," In Proceedings of the Third International Conference on Structural Engineering, Mechanics and Computation, Cape Town, South Africa (pp. 10-12).

Philippidis, T.P. and Aggelis, D.G. (2005), "Experimental study of wave dispersion and attenuation in concrete,” Ultrasonics, 43(7), pp.584-595.

Richman, M.S., Deadrick, D.S., Nation, R.J. and Whitney, S. (2001), September. "Personnel tracking using seismic sensors," In Unattended Ground Sensor Technologies and Applications III (Vol. 4393, pp. 14-21). International Society for Optics and Photonics.

Royvaran, M., Donohue, K.D. and Davis, B. (2021), "Localization of stationary source of floor vibration using steered response power method," In Dynamics of Civil Structures, Volume 2 (pp. 141-149). Springer, Cham.

Royvaran, M., Hormozabad, S.J., Soto, M.G. and Davis, B. (2020), May. "Investigation on the relationship between wave propagation speed and vibration frequency in concrete floors using nonlinear regression model," In Smart Structures and NDE for Industry 4.0, Smart Cities, and Energy Systems (Vol. 11382, p. 113820J). International Society for Optics and Photonics.

Sato, H., Fehler, M.C. and Maeda, T. (2012), "Seismic wave propagation and scattering in the heterogeneous earth,” (Vol. 496). Berlin: Springer.

Schloemann, J., Malladi, V.S., Woolard, A.G., Hamilton, J.M., Buehrer, R.M. and Tarazaga, P.A. (2015), "Vibration event localization in an instrumented building," In Experimental Techniques, Rotating Machinery, and Acoustics, Volume 8 (pp. 265-271). Springer, Cham.

Schweitzer, J. (2001), "HYPOSAT-An enhanced routine to locate seismic events," Pure and Applied Geophysics, 158(1), pp.277-289. 
Shi, G. and Ming, Y. (2016), "Survey of indoor positioning systems based on ultrawideband (UWB) technology," In Wireless Communications, Networking and Applications (pp. 1269-1278). Springer, New Delhi.

Sulaiman, A., Poletkin, K. and Khong, A.W. (2010), "Source localization in the presence of dispersion for next generation touch interface,” In 2010 International Conference on Cyberworlds (pp. 82-86). IEEE

Torrieri, D.J. (1984), “Statistical theory of passive location systems," IEEE transactions on Aerospace and Electronic Systems, (2), pp. 183-198

Tigli, O.F. (2014), "Floor vibrations on healthcare facilities: a case study on a surgical microscope,” In Dynamics of Civil Structures, Volume 4 (pp. 91-99). Springer, Cham.

Van Veen, B.D. and Buckley, K.M. (1988), "Beamforming: A versatile approach to spatial filtering,” IEEE assp magazine, 5(2), pp.4-24.

Viktorov, I.A. (1967), Rayleigh and Lamb Waves: Physical Theory and Applications: Translated from Russian. Plenum press.

Woods, R.D. and Jedele, L.P. (1985), October. "Energy—Attenuation relationships from construction vibrations," In Vibration problems in geotechnical engineering (pp. 229-246). ASCE.

Woods, R.D. (1968), Screening of suface waves in soils.

Worden, K. (2001), "Rayleigh and Lamb Waves-Basic Principles,” Strain, 37(4), pp.167172.

Yu, J. and Donohue, K.D. (2013), "Optimal irregular microphone distributions with enhanced beamforming performance in immersive environments," The Journal of the Acoustical Society of America, 134(3), pp.2066-2077.

Zheng, W.S., Gong, S. and Xiang, T. (2011), June. "Person re-identification by probabilistic relative distance comparison,” In CVPR 2011 (pp. 649-656). IEEE.

Zheng, J., Lui, K.W. and So, H.C. (2007), “Accurate three-step algorithm for joint source position and propagation speed estimation,” Signal Processing, 87(12), pp.3096-3100. 


\section{VITA}

\section{Mohammad Royvaran}

\section{EDUCATION}

- Doctor of Philosophy in Structural Engineering, University of Kentucky, Lexington, KY, Jan 2017-Jul 2021

- Master of Science in Structural Engineering, University of Kentucky, Lexington, KY, Aug 2015-Dec 2016

- Bachelor of Science in Civil Engineering, Azad University, Parand, Tehran, Iran, Sep 2006-Jan 2011

\section{PROFESSIONAL POSITION}

Design Engineer, MACNAMARA SALVIA, Boston, MA, Jul 2020-Present

\section{PUBLICATIONSS and PRESENTATION}

Royvaran, M., Avci, O. and Davis, B. (2020), “Analysis of floor vibration evaluation methods using a large database of floors framed with W-Shaped members subjected to walking excitation,” Journal of Constructional Steel Research, 164, p.105764.

Royvaran, M., Donohue, K.D. and Davis, B. (2021), “Localization of stationary source of floor vibration using steered response power method," In Dynamics of Civil Structures, Volume 2 (pp. 141-149). Springer, Cham.

Royvaran, M., Avci, O. and Davis, B. (2021), “An Overview on Floor Vibration Serviceability Evaluation Methods with a Large Database of Recorded Floor Data,” Dynamics of Civil Structures, Volume 2, pp.91-101.

Royvaran, M., Donohue, K.D. and Davis, B. (2021), "Localization of stationary source of floor vibration using steered response power method," In Dynamics of Civil Structures, Volume 2 (pp. 141-149). Springer, Cham.

Royvaran, M., Hormozabad, S.J., Soto, M.G. and Davis, B. (2020), “May. Investigation on the relationship between wave propagation speed and vibration frequency in concrete floors using nonlinear regression model," In Smart Structures and NDE for Industry 4.0, Smart Cities, and Energy Systems (Vol. 11382, p. 113820J). International Society for Optics and Photonics. 